\title{
EVALUATION OF A GROUND-WATER FLOW AND TRANSPORT MODEL OF THE UPPER COACHELLA VALLEY, CALIFORNIA
}

By Eric G. Reichard and J. Kevin Meadows

U.S. GEOLOGICAL SURVEY

Water-Resources Investigations Report 91-4142

Prepared in cooperation with the CALIFORNIA REGIONAL WATER QUALITY CONTROL BOARD, COLORADO RIVER BASIN REGION

$\frac{7}{\frac{1}{2}}$ 


\section{U.S. DEPARTMENT OF THE INTERIOR}

MANUEL LUJAN, JR., Secretary

\section{U.S. GEOLOGICAL SURVEY \\ Dallas L. Peck, Director}

Any use of trade, product, or firm names in this publication is for descriptive purposes only and does not imply endorsement by the U.S. Government.

For sale by the Books and Open-File Reports Section, U.S. Geological Survey

Federal Center, Box 25425

Denver, CO 80225

For additional information write to:

District Chief

U.S. Geological Survey

Federal Building, Room W-2234

2800 Cottage Way

Sacramento, CA 95825 


\section{CONTENTS}

Abstract 1

Introduction 1

Purpose and scope 2

Acknowledgments 2

Well-numbering system 2

Description of study area 3

Geographic setting 3

Climate 3

Geohydrology 3

Changes in ground-water conditions since previous (1978) model study 7

Hydrologic data base 7

Ground-water use 7

Artificial recharge 7

Underflow and recharge from streams in upper Coachella Valley 7

Ground-water levels 10

Regional ground-water quality 10

Evaluation of existing model $\mathbf{1 5}$

Description of model 15

Conversion of existing model to the computer code SUTRA 15

Hydrogeologic parameters used in existing model 17

Reproduction, using SUTRA, of previous existing-model simulations 17

New transient simulations for 1979-86 17

Solute-transport simulations for 1979-86 27

Conclusions 40

Model evaluation $\mathbf{4 0}$

Future data collection 41

Future modeling 41

References cited $\mathbf{4 2}$

Appendixes

1. List and description of wells in data base $\mathbf{4 3}$

2. Gross pumpage for January-June and July-December, 1979-86, for wells in data base 56

3. Ground-water levels, autumn 197863

4. Ground-water levels, autumn 198667

5. Dissolved-solids concentration in water from selected wells $\mathbf{7 1}$

6. Modifications to SUTRA to allow input of spatially varying aquifer-storage coefficient 79

7. Specified-head values used for southeast boundary of model $\mathbf{8 2}$

8. SUTRA unit 55 input file for simulations shown in figures $14-16,18$, and 1983

9. SUTRA unit 5 input file for simulations shown in figures 18 and 1986 


\section{FIGURES}

1. Map showing study area and location of faults, geohydrologic section, and area of ground-water model 4

2. Generalized geohydrologic section of Coachella Valley, showing electricalresistivity logs in the unconsolidated deposits 6

3. Map showing location of wells in data base $\mathbf{8}$

4. Graph showing net flux, net pumpage, and diversions to Whitewater River in the upper Coachella Valley, 1936-86 9

5. Hydrographs showing water levels for selected wells in the upper Coachella Valley 10

6. Graphs showing dissolved-solids concentration in water from selected wells in the upper Coachella Valley 12

7. Map showing dissolved-solids concentration in water from selected wells near the artificial-recharge area, autumn 198614

8-13. Maps showing:

8. Model mesh, source/sink nodes, boundary conditions, and monitoring-well locations for the SUTRA model, and location of solute-transport submodel 16

9. Aquifer saturated thickness in the model area $\mathbf{1 8}$

10. Hydraulic conductivity in the model area 19

11. Storage-coefficient distribution used in existing model $\mathbf{2 0}$

12. Simulated steady-state (1936) water levels using SUTRA model 21

13. Simulated water-level change, 1974-80, using SUTRA model 22

14-16. Hydrographs showing simulated water levels, 1979-86, and measured water levels at selected wells:

14. Using all parameters from existing model 24

15. Using all parameters from existing model, except assuming 350 -foot increase in saturated thickness of aquifer in the artificial-recharge area 25

16. Using all parameters from existing model, except assuming 350 -foot increase in saturated thickness of aquifer in the artificial-recharge area and assuming a maximum of 150,000 acre-feet per year of artificial recharge 26

17,18. Maps showing:

17. Revised storage-coefficient distribution used in SUTRA model 28

18. Simulated water levels, with revised storage-coefficient distribution used in SUTRA model, and measured water levels, autumn 198629

19. Hydrographs showing simulated water levels, 1979-86, with revised storagecoefficient distribution used in SUTRA model, and measured water levels at selected wells $\mathbf{3 0}$

20. Graphs showing cumulative annual flow and cumulative departure from mean annual flow for three Coachella Valley streams 31

21. Map showing simulated and measured dissolved-solids concentration, autumn 1986, for base-case simulation using the solute-transport submodel 32 
22. Graphs showing simulated dissolved-solids concentration, 1979-86, for base-case simulation, and measured dissolved-solids concentration in water from selected wells 33

23. Generalized geohydrologic section showing perforated intervals of wells near the artificial-recharge area $\mathbf{3 5}$

24. Map showing simulated dissolved-solids concentration, assuming that all transport occurs in the upper 20 percent of the aquifer, and measured dissolved-solids concentration, autumn 198637

25. Graphs showing simulated dissolved-solids concentration, 1979-86, assuming that all transport occurs in the upper 20 percent of the aquifer, and measured dissolved-solids concentration at selected wells $\mathbf{3 8}$

\section{TABLES}

1. Annual quantities and assumed dissolved-solids concentration of Colorado River water released to Whitewater River, 1973-87, and model-input values for artificial recharge, 1979-86 9

2. Estimated long-term average rates of ground-water recharge in upper Coachella Valley 9 
CONVERSION FACTORS, ABBREVIATIONS, AND VERTICAL DATUM

$\begin{array}{lll}\text { Multiply } & \text { By } & \text { To obtain }\end{array}$

Temperature is given in degrees Fahrenheit $\left({ }^{\circ} \mathrm{F}\right)$, which is converted to degrees Celsius $\left({ }^{\circ} \mathrm{C}\right)$ by using the formula:

$$
\text { Temp. }{ }^{\circ} \mathrm{C}=\left(\text { temp. }{ }^{\circ} \mathrm{F}-32\right) / 1.8 .
$$

\section{Abbreviations and Acronyms Used}

$\mathrm{mg} / \mathrm{L} \quad-$ milligram per liter

SUTRA - Saturated-Unnsaturated TRAnsport--a computer program for simulating subsurface fluid movement and energy or solute transport

\section{Vertical Datum}

"Sea level" in this report refers to the National Geodetic Vertical Datum of 1929 (NGVD of 1929)--a geodetic datum derived from a general adjustment of the first-order level nets of both the United States and Canada, formerly called Sea Level Datum of 1929. 


\title{
EVALUATION OF A GROUND-WATER FLOW AND TRANSPORT MODEL IN THE UPPER COACHELLA VALLEY, CALIFORNIA
}

\author{
By Eric G. Reichard and J. Kevin Meadows
}

\begin{abstract}
A geohydrologic data base was compiled to evaluate the changes in ground-water conditions in the upper Coachella Valley, California, since 1978, when the results of a previous ground-water-model study were published. The compiled data were analyzed to improve understanding of the ground-water flow system and the effects on water quality of an existing artificial-recharge program. Analysis of well logs indicates that several zones of differing permeability can be correlated throughout the valley. Analysis of the water-quality data suggests that the artificial recharge of Colorado River water has caused increases in dissolved solids in some wells. Sparse data indicate that the recharged water moves much more rapidly through the uppermost part of the ground-water system than through deeper parts.
\end{abstract}

An evaluation of the existing ground-water simulation model of the upper Coachella Valley that was developed in the 1978 study yielded several conclusions. First, the 300 - to 400 -foot rise in water levels in the artificialrecharge area in 1985-86 significantly affected the groundwater flow system. Transmissivities in the model had to be increased to reflect the increase in saturated thickness. Storage-coefficient values in the model had to be increased from 0.02 (used in the previous study) to $0.10-0.15$ to simulate the unconfined water-level response during this period. Second, stream-recharge and underflow rates from San Gorgonio Pass during 1979-86 seem to have been higher than the values estimated for previous studies. Conditions generally were wetter in 1979-86 than during the period in which the earlier estimates were made. Third, the flow system could be simulated more accurately if the model were extended to its true southeastern boundary, the Salton Sea. Finally, there are important vertical differences in transport of the artificially recharged water that the two-dimensional areal transport model cannot simulate.

The study results indicate several additional datacollection and modeling needs. The drilling of several sets of monitor wells perforated at different depths could help better define the three-dimensional flow system and waterquality differences. Additional data also are needed on underflow and stream recharge, evaporation rates and infiltration rates at the artificial-recharge facilities, and net pumpage. Three-dimensional flow and transport models are needed to evaluate the effects of ground-water development on water levels and water quality.

\section{INTRODUCTION}

The Coachella Valley in southern California is an arid basin facing continuing population growth and increases in ground-water demand. Since 1973, water from the Colorado River Aqueduct has been artificially recharged to the ground-water system in the upper Coachella Valley. Ground-water flow and transport models of the upper Coachella Valley are potentially useful for (1) simulating the effects of the existing artificial-recharge program on water levels and water quality; (2) predicting the effects on the ground-water system of alternative scenarios of future water development; (3) increasing understanding of the hydrogeologic system; and (4) providing a guide for data collection.

As a result of these considerations, the U.S. Geological Survey, in cooperation with the California Regional Water Quality Control Board, Colorado River Basin Region, conducted a study to evaluate the existing groundwater simulation model of the upper Coachella Valley and to identify future data-collection and modeling needs. 


\section{Purpose and Scope}

This report describes an evaluation of the existing ground-water simulation model of the upper Coachella Valley developed by Swain (1978). The evaluation consisted of several steps. First, an updated geohydrologic data base was compiled to evaluate changes in groundwater conditions since publication of the results of Swain's study. Second, the compiled data were analyzed to characterize the ground-water flow system and to quantify the effects on water quality of the artificial-recharge program during
1979-86. Third, the existing model was used to simulate ground-water flow and solute transport for the period 1979-86. The results of these simulations helped identify future modeling and data-collection needs for the valley.

\section{Acknowledgments}

The assistance of the Desert Water Agency and the Coachella Valley Water District in providing data for this study is greatly appreciated.

\section{Well-Numbering System}

Wells are numbered according to their location in the rectangular system for subdivision of public land. For example, in the well number 3S/4E-20D1, the part of the number preceding the slash indicates the township (T. $3 \mathrm{~S}$.); the number following the slash indicates the range (R. 4 E.); the number following the hyphen indicates the section (sec. 20 ); and the letter following the section number indicates the 40 -acre subdivision according to the lettered diagram below. The final digit is a serial number for wells in each 40 -acre subdivision. The entire study area is south and east of the San Bernardino baseline and meridian. The diagram shows the location of a well with the well number 3S/4E-20D1.

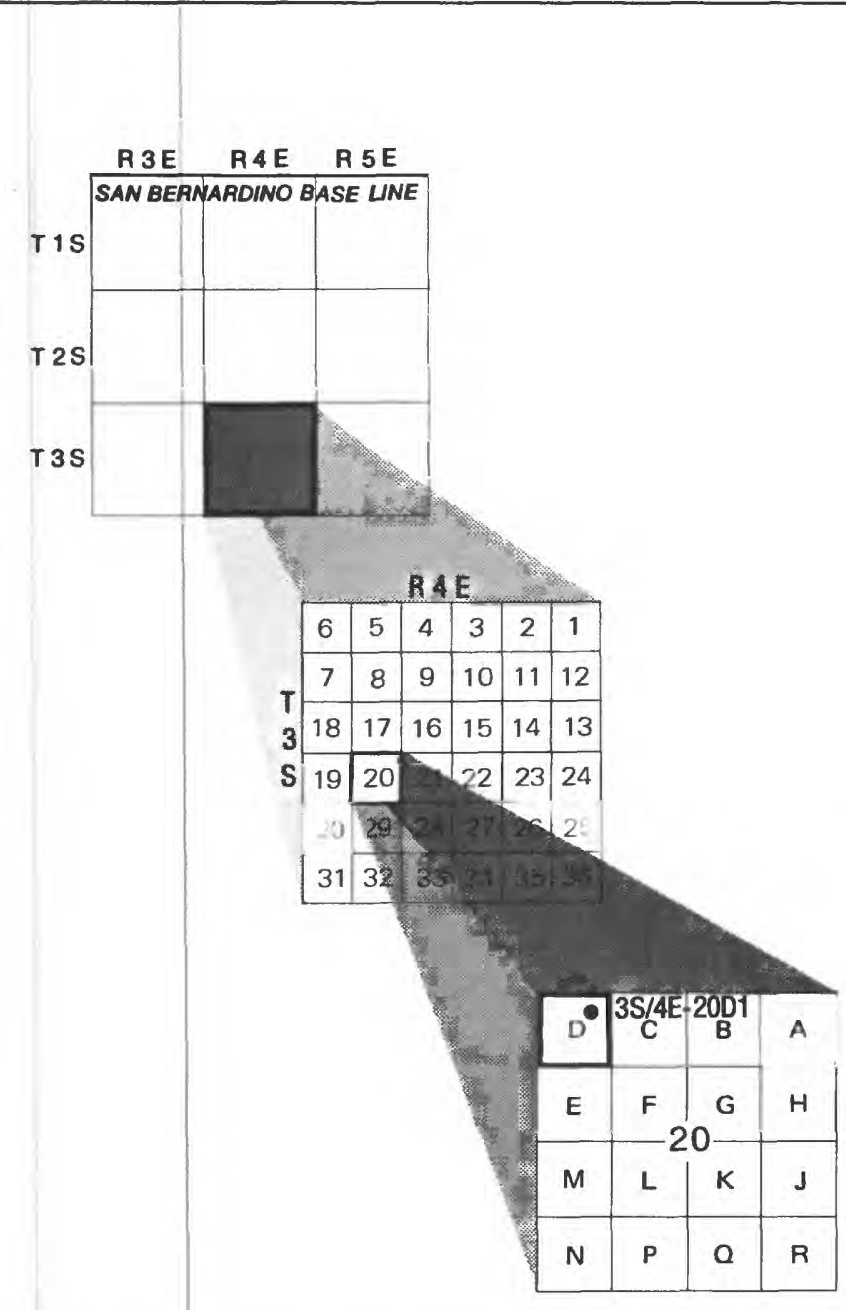




\section{DESCRIPTION OF STUDY AREA}

\section{Geographic Setting}

The Coachella Valley is mostly in central Riverside County, extending into small parts of San Bernardino County on the north and San Diego and Imperial Counties on the south. The northwest-southeast-trending valley is about 65 mi long and includes about $440 \mathrm{mi}^{2}$. The valley is drained primarily by the Whitewater River system, which empties into the Salton Sea (fig. 1). Total drainage area of the Whitewater River is about $1,500 \mathrm{mi}^{2}$, and the altitude of the drainage basin ranges from more than $10,000 \mathrm{ft}$ above sea level at the peaks of the San Bernardino and San Jacinto Mountains to more than $200 \mathrm{ft}$ below sea level at the Salton Sea.

The models described in this report cover only that part of the upper Coachella Valley southwest of the Banning and San Andreas faults (see fig. 1). As defined in this report, the upper Coachella Valley extends from the San Gorgonio Pass southeastward to about $2 \mathrm{mi}$ north of the city of Indio, and the lower Coachella Valley extends southeastward from the southern boundary of the modeled area to the Salton Sea.

\section{Climate}

Climate of the valley is typical of a desert environment. Average annual precipitation on the valley floor is less than $5 \mathrm{in}$., but in the mountains west and north of the valley it is as much as $40 \mathrm{in}$. Temperatures range from about $120^{\circ} \mathrm{F}$ in summer on the valley floor to below freezing in winter in the bordering mountains.

\section{Geohydrology}

Several reports on the geology of the Coachella Valley and aspects of its geohydrology have been published. Mendenhall (1909) provided a general description of the geology and water resources of the region. He showed that the source of recharge to the ground-water system is precipitation and mountain-front runoff rather than some distant source such as infiltration of water from the Colorado River, as was commonly believed at the time. Brown (1923) presented a qualitative overview of the geology and hydrology of the entire Salton Sea region. Huberty and others (1948) compiled a useful survey of water levels and water quality. Proctor (1968) described the lithology of the units in the surrounding mountains and the stratigraphy of the valley deposits, and provided a literature review of geologic studies of the entire Colorado Desert area. The most comprehensive summary of the hydrology of the Coachella Valley was completed by the California Department of Water Resources (1964). Loeltz and others (1975) provided a discussion of the regional geologic setting. The California Department of Water Resources (1979) compared water quality in wells of different depths in the Coachella Valley, focusing on the potential effects of poorly constructed or abandoned wells.

The Coachella Valley is part of the Salton Trough of the Basin and Range province. The Salton Trough, which is the landward extension of the Gulf of California, was formed in late Cenozoic time (Loeltz and others, 1975). Both Coachella Valley and Imperial Valley (south of the Salton Sea) drain into the lowest part of the trough, the Salton Sea.

The Coachella Valley is filled with as much as $12,000 \mathrm{ft}$ of sediments (California Department of Water Resources, 1979). It is bounded by the pre-Tertiary igneous and metamorphic rocks of the San Bernardino and Little San Bernardino Mountains to the northeast and the San Jacinto and Santa Rosa Mountains to the southwest. The major faults in the Coachella Valley--the San Andreas, Banning, Garnet Hill, Mission Creek, and Indio Hills faults (see fig. 1)--are part of the San Andreas fault system. 


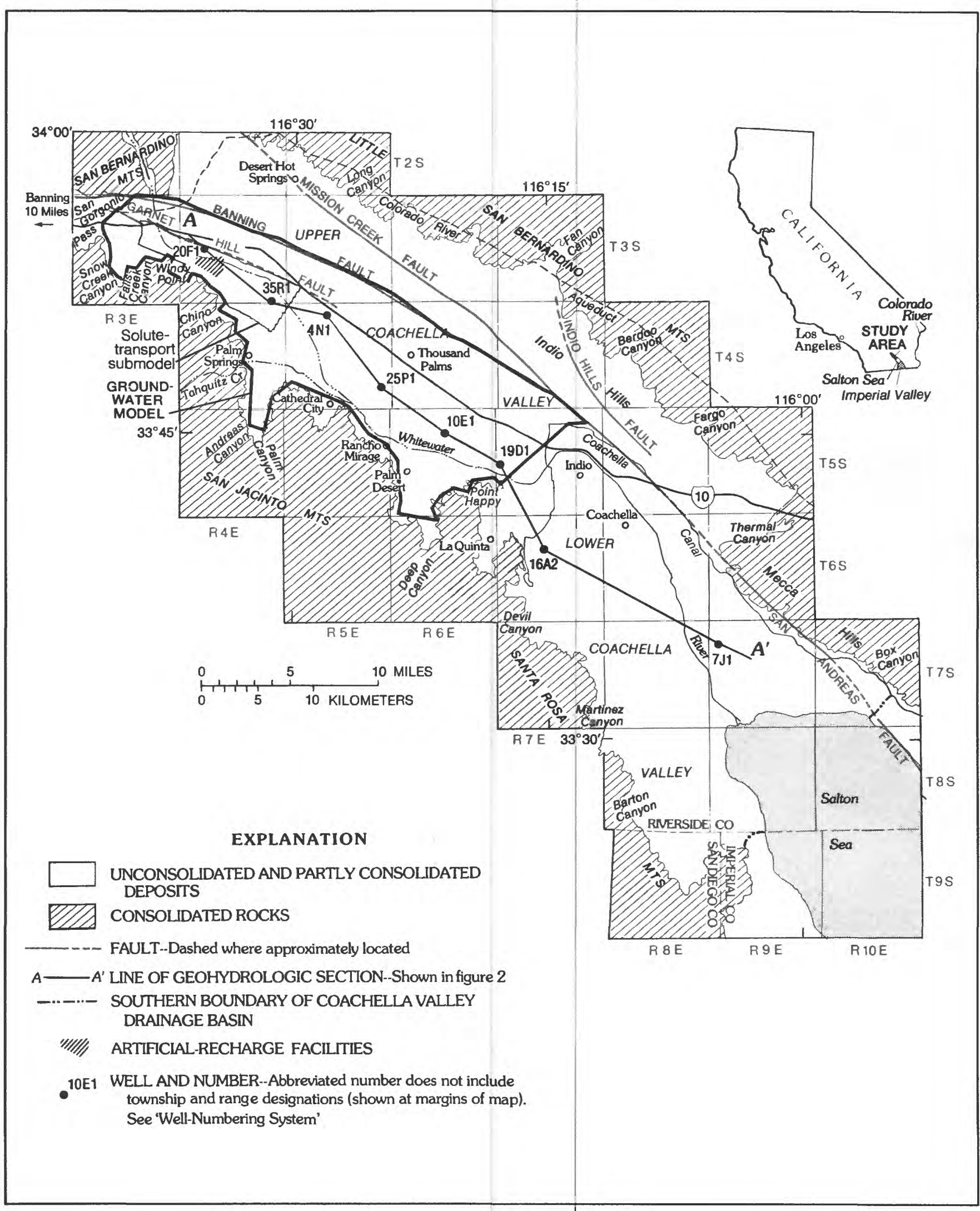

Figure 1. Study area and location of faults, geohydrologic section, and area of ground-water model. 
These faults impede the flow of ground water in the valley (California Department of Water Resources, 1964).

As stated above, the models presented in this report cover only a part of the upper Coachella Valley. The modeled area is bounded on the southwest by the San Jacinto and Santa Rosa Mountains, on the northeast by the Banning and San Andreas faults, and on the northwest by the San Gorgonio Pass. The consolidated rocks of the San Jacinto and Santa Rosa Mountains act as barriers to flow of ground water. Ground water flows into the modeled area from the San Gorgonio Pass and, to a much lesser extent, across the Banning fault. The southeast boundary of the modeled area is a line extending from Point Happy to the San Andreas fault. This is the line that was chosen by Tyley (1974) and Swain (1978) to represent the boundary between the upper Coachella Valley, where ground-water levels historically were declining, and the lower Coachella Valley, where ground-water levels had been rising since completion of the Coachella Canal in 1949.

Tyley (1974) divided the geologic units in the modeled area into consolidated, semiconsolidated (partly consolidated), and unconsolidated deposits. The pre-Tertiary and Tertiary consolidated deposits, which are considered as rocks yielding no water to wells, form the basement of the modeled area and the adjacent San Jacinto and Santa Rosa Mountains. The Pliocene and Pleistocene partly consolidated deposits overlying the Tertiary deposits are exposed in the Indio Hills. The partly consolidated deposits yield little water. The Pleistocene and Holocene unconsolidated deposits are the main water-bearing units. The most significant water-bearing units are the Ocotillo Conglomerate, described by Dibblee (1954) as upper Pliocene or lower Pleistocene, the Cabezon Fanglomerate of Pleistocene age, and the Pleistocene and Holocene alluvium (California Department of Water Resources, 1964).
The Ocotillo Conglomerate is described by the California Department of Water Resources (1964) as consisting of poorly consolidated sandstones and conglomerates with lenses of gray-green and red-brown silt and clay. The Cabezon Fanglomerate, considered correlative with the Ocotillo Conglomerate, was deposited at the base of the San Bernardino Mountains and is present in the northwestern part of the valley (California Department of Water Resources, 1964). This unit is described by Allen (1957) as a gray-to-tan poorly sorted conglomerate with abundant granitic boulders.

The total thickness of the water-bearing units--the Ocotillo Conglomerate, the Cabezon Fanglomerate, and the Pleistocene and Holocene alluvium--is estimated to be as much as 2,000 ft in the Coachella Valley (California Department of Water Resources, 1979). For modeling purposes, Tyley (1974) and Swain (1978) assumed the thickness of the active flow system in the water-bearing deposits to be about 1,000 ft. This assumption was based on the decreasing resistivity with increasing depth observed in geophysical logs and on the fact that maximum well depths in the Coachella Valley are 1,000 to $1,200 \mathrm{ft}$.

The water-bearing deposits in the modeled area are heterogeneous areally and vertically. A generalized geologic section of the Coachella Valley (fig. 2) shows electrical-resistivity logs in the unconsolidated deposits. Although the data are sparse and the correlations drawn are subjective, it appears that several layers of differing electrical resistivity in the unconsolidated deposits can be traced throughout the length of the valley. These layers may or may not be continuous. Low-resistivity units generally represent zones of lower permeability such as clay and silt. High-resistivity units generally represent zones of higher permeability such as sand and gravel. (It is not possible to distinguish between the Ocotillo Conglomerate, the Cabezon Fanglomerate, and the Pleistocene and Holocene alluvium in figure 2.) Two zones 


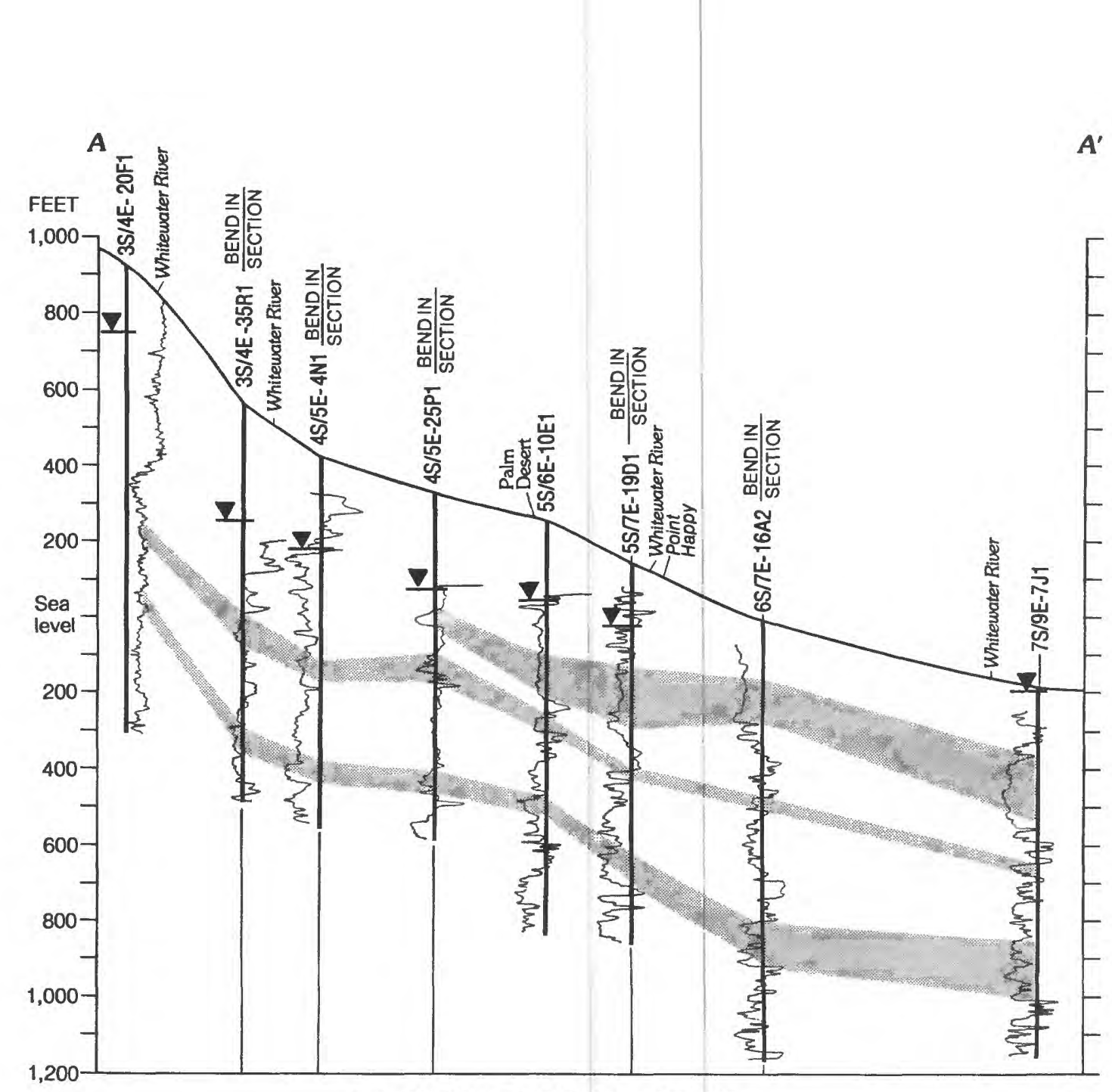

VERTICAL SCALE GREATIY EXAGGERATED

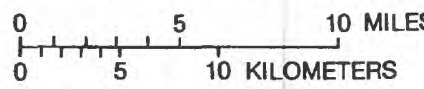

EXPLANATION

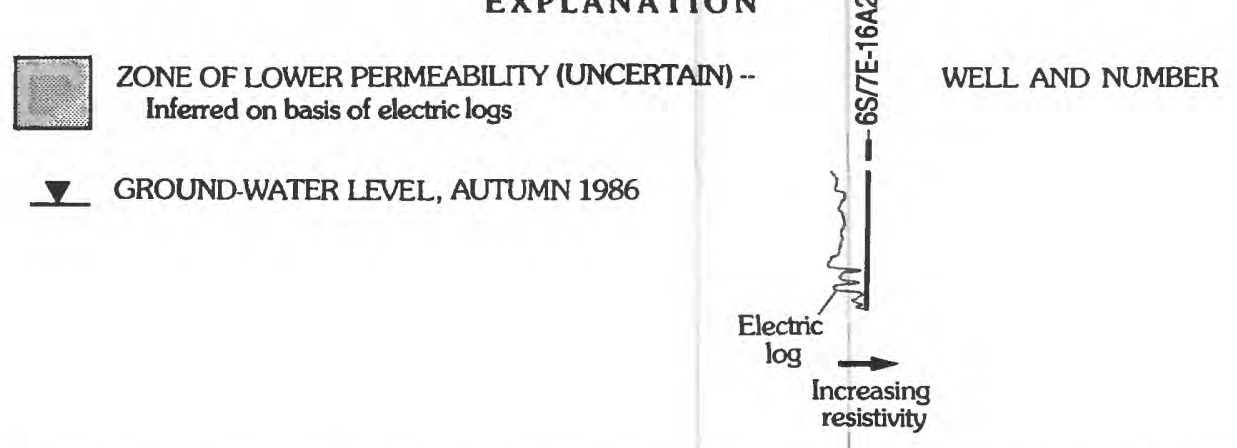

Figure 2. Generalized geohydrologic section of Coachella Valley, showing electrical-resistivity logs in the unconsolidated deposits.

of lower permeability were identified in the upper valley. A third zone of lower permeability is present near the middle of the section north of Palm Desert. These zones of lower permeability may impede vertical ground-water flow and chemical transport. 


\section{CHANGES IN GROUND-WATER CONDITIONS SINCE PREVIOUS (1978) MODEL STUDY}

\section{Hydrologic Data Base}

In order to evaluate the changes in groundwater conditions in the Coachella Valley since the previous model study (Swain, 1978), it first was necessary to compile an updated hydrologic data base. The computerized data base includes digitized well locations, water levels, landsurface altitudes, perforated intervals, and pumping rates for about 600 wells. Location of the wells is shown in figure 3 . Water-quality information for 38 wells and measured streamflows and artificial-recharge quantities also are stored in the data base. Data on several other geohydrologic features, including surficial geology, streams, and recharge areas, have been digitized and incorporated into the data base. The computer data base allows easy interaction with ground-water simulation models and graphic packages. For example, water-level data can be extracted to produce contour maps and hydrographs. All wells in the data base are tabulated in appendix 1.

Additional information has been compiled and filed in the San Diego Office of the U.S. Geological Survey. This information includes well logs (about 220 drillers' logs and 35 geophysical logs), land use, population projections, flow in the Coachella Canal, and discharge permits.

\section{Ground-Water Use}

Estimated historical (1936-86) net groundwater pumpage for the upper Coachella Valley is shown in figure 4. Net pumpage is defined as the quantity of water actually removed from the ground-water system: that is, net pumpage equals gross pumpage minus nonused water that infiltrates back into the ground-water system.
Values for 1936-73 were estimated by Tyley (1974) and Swain (1978). Values for 1979-86 were compiled in this study from gross-pumpage data provided by the Coachella Valley Water District and the Desert Water Agency. Net pumpage was assumed to be 65 percent of gross pumpage. This value (of 65 percent) was based on a study by the California Department of Water Resources $(1987$; 1988; written commun., 1989) and is subject to a great deal of uncertainty. As shown in figure 4, estimated annual net pumpage in the upper valley has increased more than 50 percent from 1979 to 1986 (from 63,000 acre-ft to 98,000 acre-ft). Gross pumpage for 1979-86 for all wells in the data base is tabulated in appendix 2 .

\section{Artificial Recharge}

Since 1973, water from the Colorado River Aqueduct has been artificially recharged to the ground-water system through recharge facilities at the upper end of the valley (see fig. 1). The water is released to the Whitewater River, then diverted to recharge facilities that consist of approximately 700 acres of percolation ponds. The total volume of water diverted to the percolation ponds from 1973 to 1987 is about 960,000 acre-ft; of this total, about 650,000 acre-ft was diverted during 1985-87. During those 3 years, ground-water levels in the artificial-recharge area rose about 300 to $400 \mathrm{ft}$. No water was diverted to the recharge facilities from spring 1987 through autumn 1989. Annual quantities of Colorado River water released to the Whitewater River for artificial recharge are shown in figure 4 and listed in table 1.

Underflow and Recharge from Streams in Upper Coachella Valley

Estimates of long-term average annual underflow and recharge from streams (Swain, 1978) for the Coachella Valley are given in 


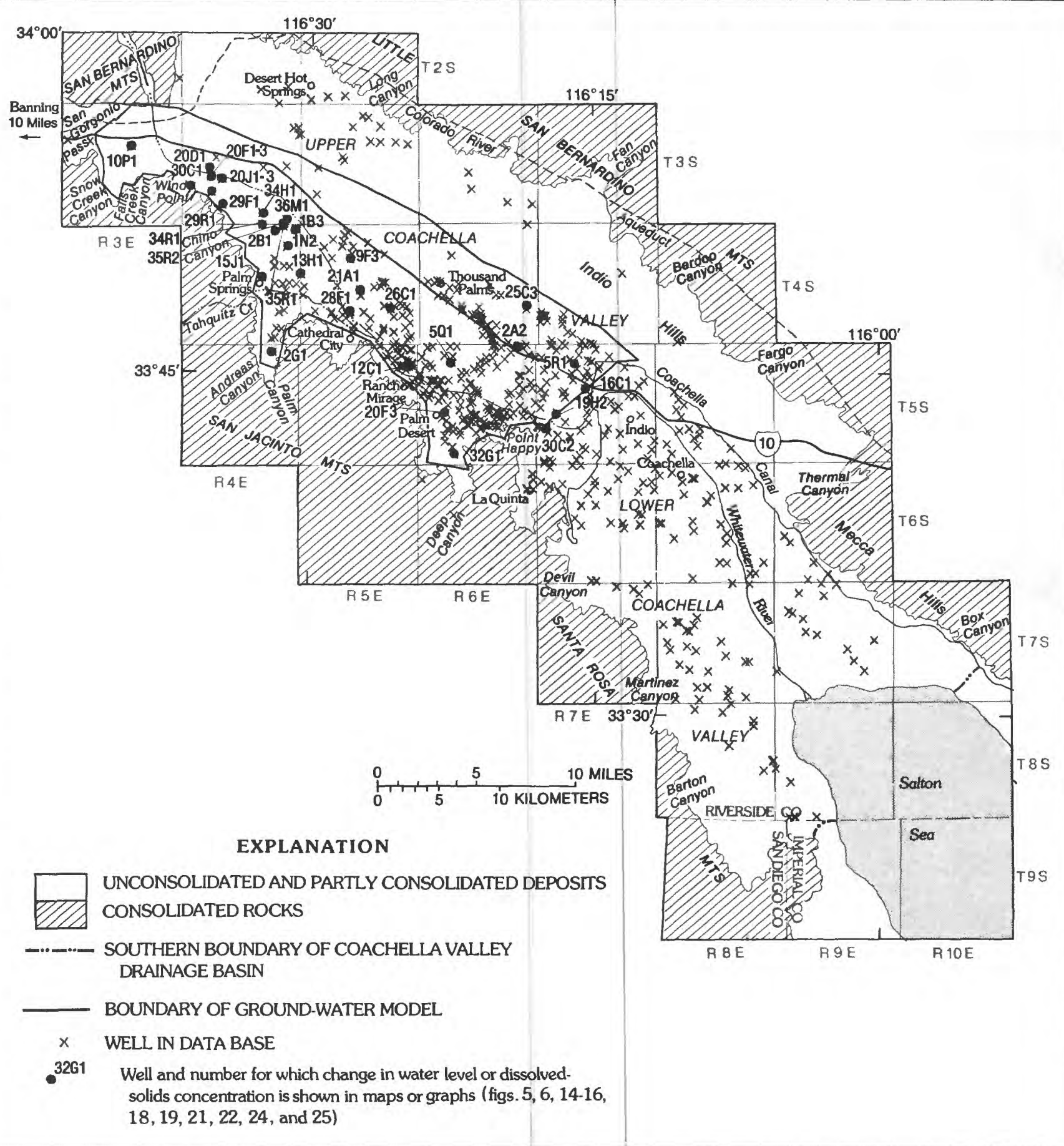

Figure 3. Location of wells in data base.

table 2. These rates, as discussed later, are smaller than the recharge rates for 1979-86. The estimated average rate of subsurface outflow from the upper valley to the lower valley, on the basis of the boundary fluxes computed by the simulations completed during this study, is about 7,000 acre-ft/yr.
Net flux in the upper valley during the simulation period, 1979-86, is shown in figure 4. Net flux equals the sum of artificial recharge, stream recharge, and underflow from San Gorgonio Pass and across the Banning fault, minus net pumpage and subsurface outflow to the lower valley. 
Table 1. Annual quantities and assumed dissolved-solids concentration of Colorado River water released to Whitewater River, 1973-87, and model-input values for artificial recharge, 1979-86

[Assumed average dissolved-solids concentration based on measurements at Lake Havasu and Lake Mathews (Metropolitan Water District, oral commun., 1990). Acre-ft, acre-feet; mg/L, milligrams per liter; --, not applicable]

\begin{tabular}{|c|c|c|c|c|c|}
\hline Calendar year & $\begin{array}{l}\text { Water diverted } \\
\text { (acre-ft) }\end{array}$ & $\begin{array}{c}\text { Assumed average } \\
\text { dissolved-solids } \\
\text { concentration }(\mathrm{mg} / \mathrm{L})\end{array}$ & $\begin{array}{l}\text { Amount applied to } \\
\text { Whitewater River } \\
\text { channel (nodes } 35,38 \text {, } \\
42,50,57,68 \text { ) (acre-ft) }\end{array}$ & $\begin{array}{l}\text { Assumed evapora- } \\
\text { tion (acre-ft) }\end{array}$ & $\begin{array}{c}\text { Amount applied to } \\
\text { recharge ponds } \\
\text { (nodes } 76,77,78,84, \\
85,86,92,93,94 \text { ) } \\
\text { (acre-ft) }\end{array}$ \\
\hline 1973 & 7,000 & No data & - & -- & -- \\
\hline 1974 & 15,000 & 710 & - & - & -- \\
\hline 1974 & 20,000 & 710 & - & -- & -- \\
\hline 1978 & 0 & -- & - & -- & -- \\
\hline 1979 & 25,000 & 700 & 5,000 & 4,000 & 16,000 \\
\hline 1980 & 26,000 & 700 & 5,200 & 4,000 & 16,800 \\
\hline 1981 & 35,000 & 700 & 7,000 & 4,000 & 24,000 \\
\hline 1982 & 27,000 & 690 & 5,400 & 4,000 & 17,600 \\
\hline 1983 & 54,000 & 680 & 10,800 & 4,000 & 39,200 \\
\hline
\end{tabular}

Table 2.--Estimated long-term average rates of ground-water recharge in upper Coachella Valley

[From Swain, 1978]

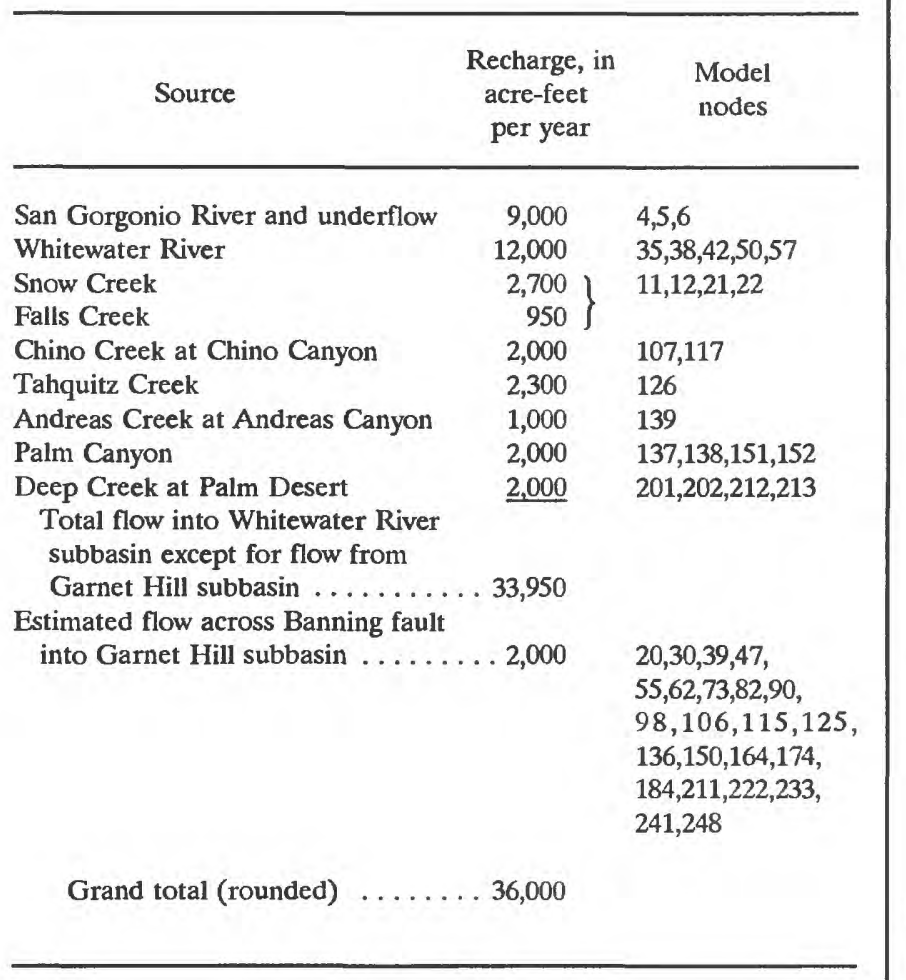

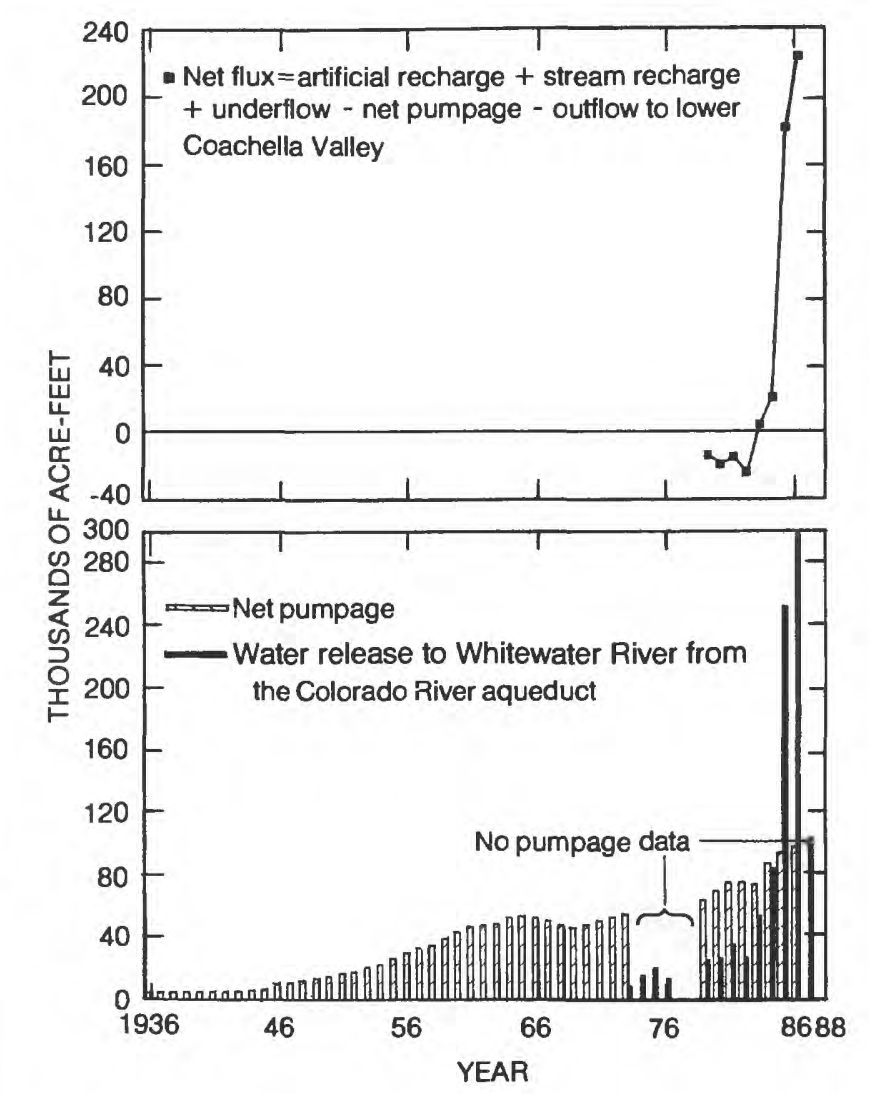

Figure 4. Net flux, net pumpage, and diversions to Whitewater River in the upper Coachella Valley, 1936-86. 


\section{Ground-Water Levels}

Water-level hydrographs of selected wells in the upper valley are shown in figure 5, and water levels tabulated by well for autumn 1978 and autumn 1986 are given in appendixes 3 and 4 , respectively. Water levels in the northern part of the upper valley (for example, wells [location shown in fig. 3] in townships $3 \mathrm{~S} / 3 \mathrm{E}$, $3 \mathrm{~S} / 4 \mathrm{E}, 4 \mathrm{~S} / 4 \mathrm{E}$, and $4 \mathrm{~S} / 5 \mathrm{E}$ ) rose during 1979-86 as a result of the artificial-recharge program, increased underflow from San Gorgonio Pass, and increased mountain-front recharge. Water levels in the southern part of the upper valley (for example, wells in townships $5 \mathrm{~S} / 4 \mathrm{E}, 5 \mathrm{~S} / 6 \mathrm{E}$ and 5S/7E) generally declined during 1979-86. Note particularly that wells $5 \mathrm{~S} / 7 \mathrm{E}-16 \mathrm{C} 1,-19 \mathrm{H} 2$, and $-30 \mathrm{C} 2$ at the southern boundary of the model area have been declining at an average rate of 1 to $3 \mathrm{ft} / \mathrm{yr}$. In the original modeling studies (Tyley, 1974; Swain, 1978), hydraulic heads in this area were assumed to be constant.

\section{Regional Ground-Water Quality}

A regional water-quality issue in the upper valley is the effect of the artificially recharged Colorado River water on the quality of the native ground water. The Colorado River water has a significantly higher dissolved-solids concentration (greater than $500 \mathrm{mg} / \mathrm{L}$--see table 1) than the native water (about $200 \mathrm{mg} / \mathrm{L}$ ). Dissolved-solids concentration of ground water near the artificial-recharge area generally has increased as a result of the artificial recharge (fig. 6). A map of dissolved-solids concentration of ground water sampled in autumn 1986 (fig. 7) shows that wells near each other may have markedly different dissolved-solids concentrations. As is discussed in more detail in the section, "Solute-Transport Simulations for 197986 ," these concentration differences may be due to vertical differences in solute-transport rates. Dissolved-solids concentrations tabulated by well are shown in appendix 5.
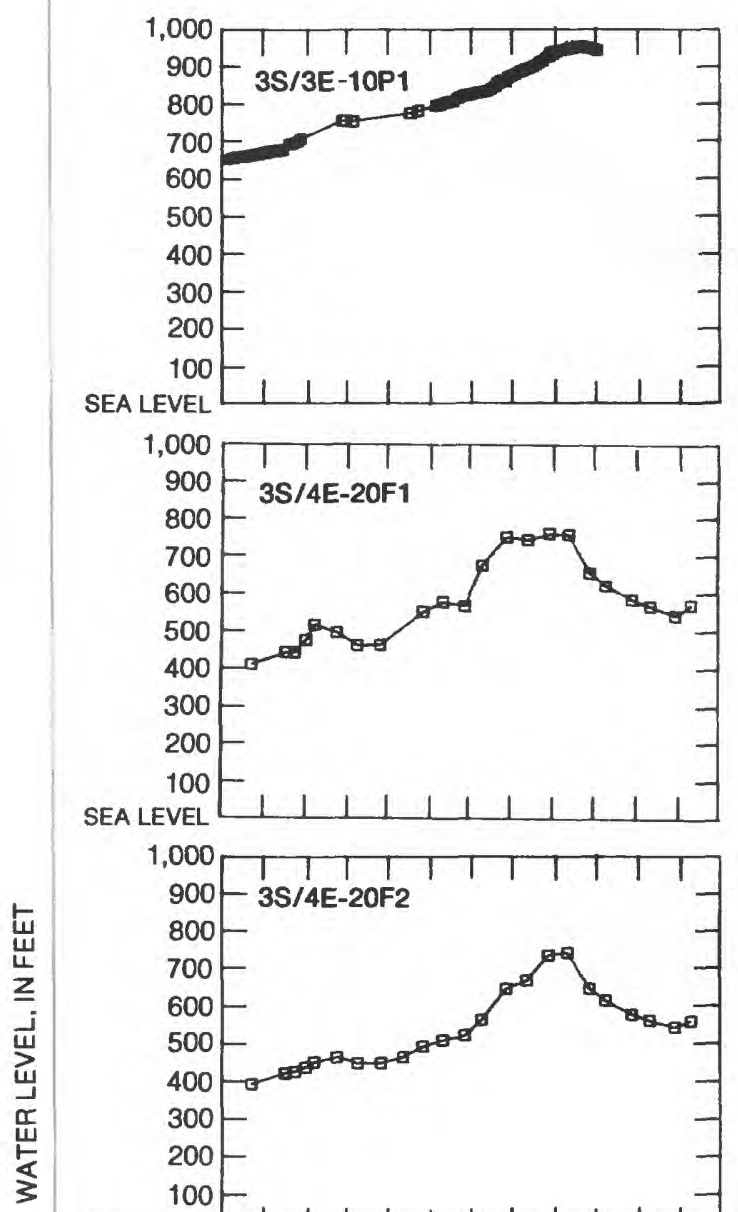

荘

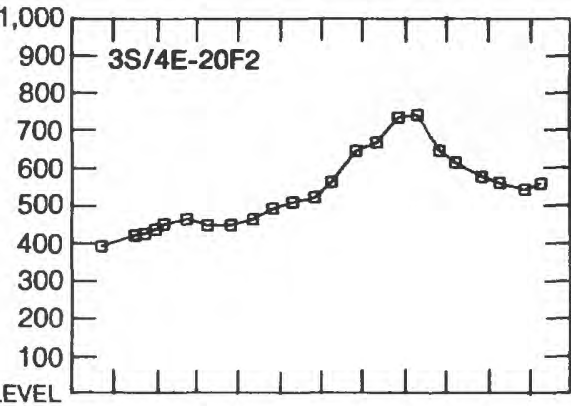

SEA LEVE
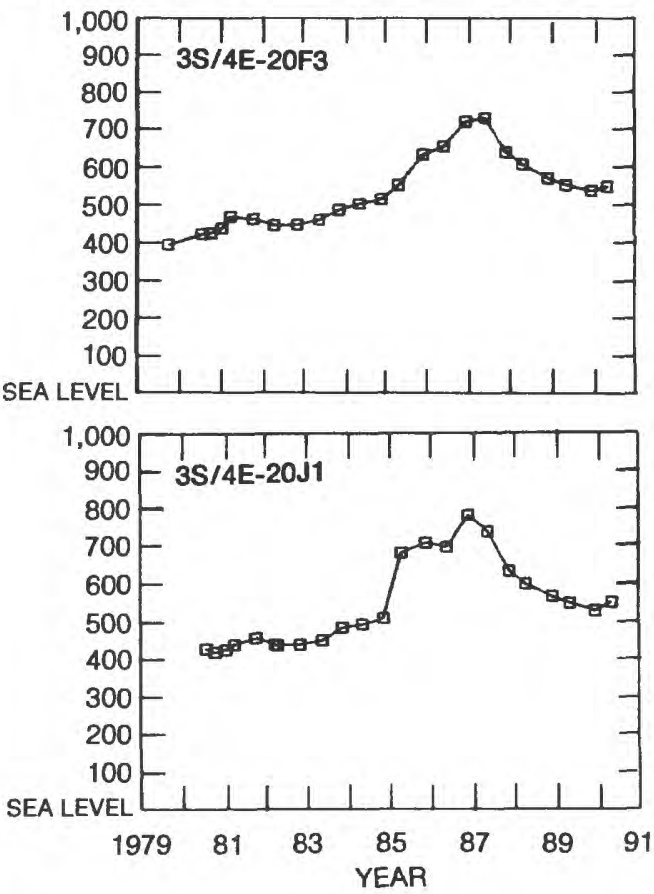

Figure 5. Water levels for selected wells in the upper Coachella Valley. 


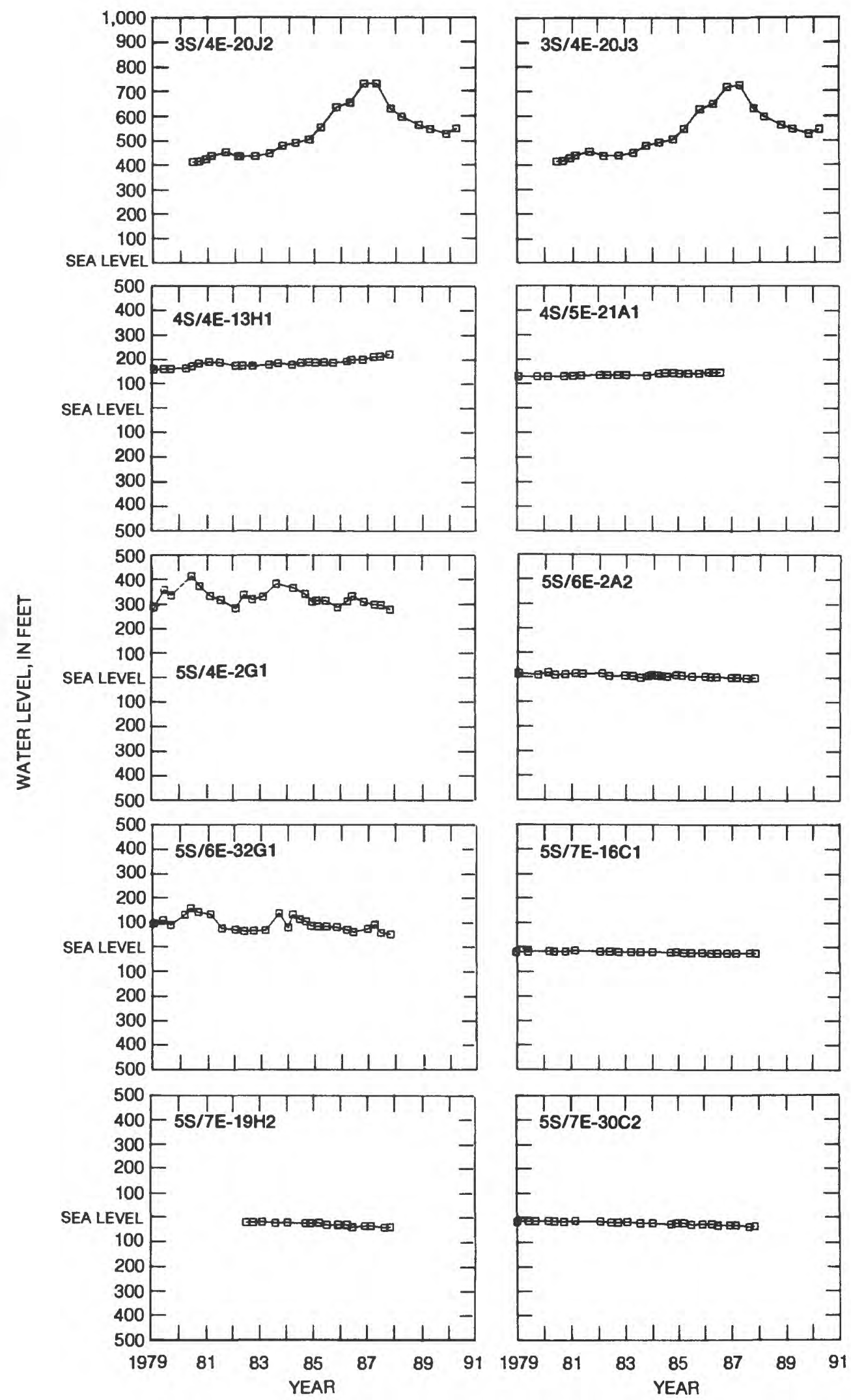

Figure 5. Continued. 

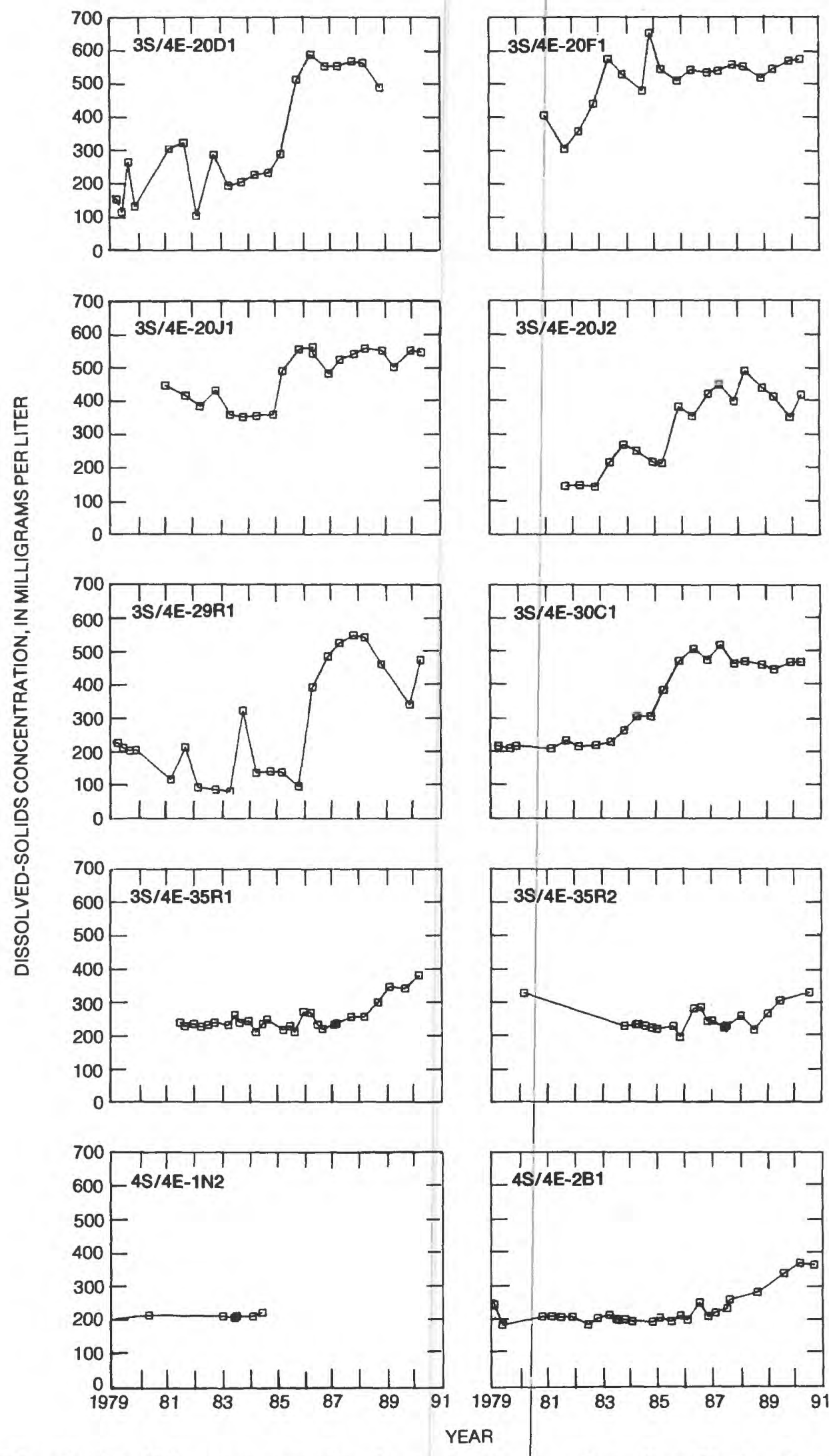

Figure 6. Dissolved-solids concentration in water from selected wells in the upper Coachella Valley. 


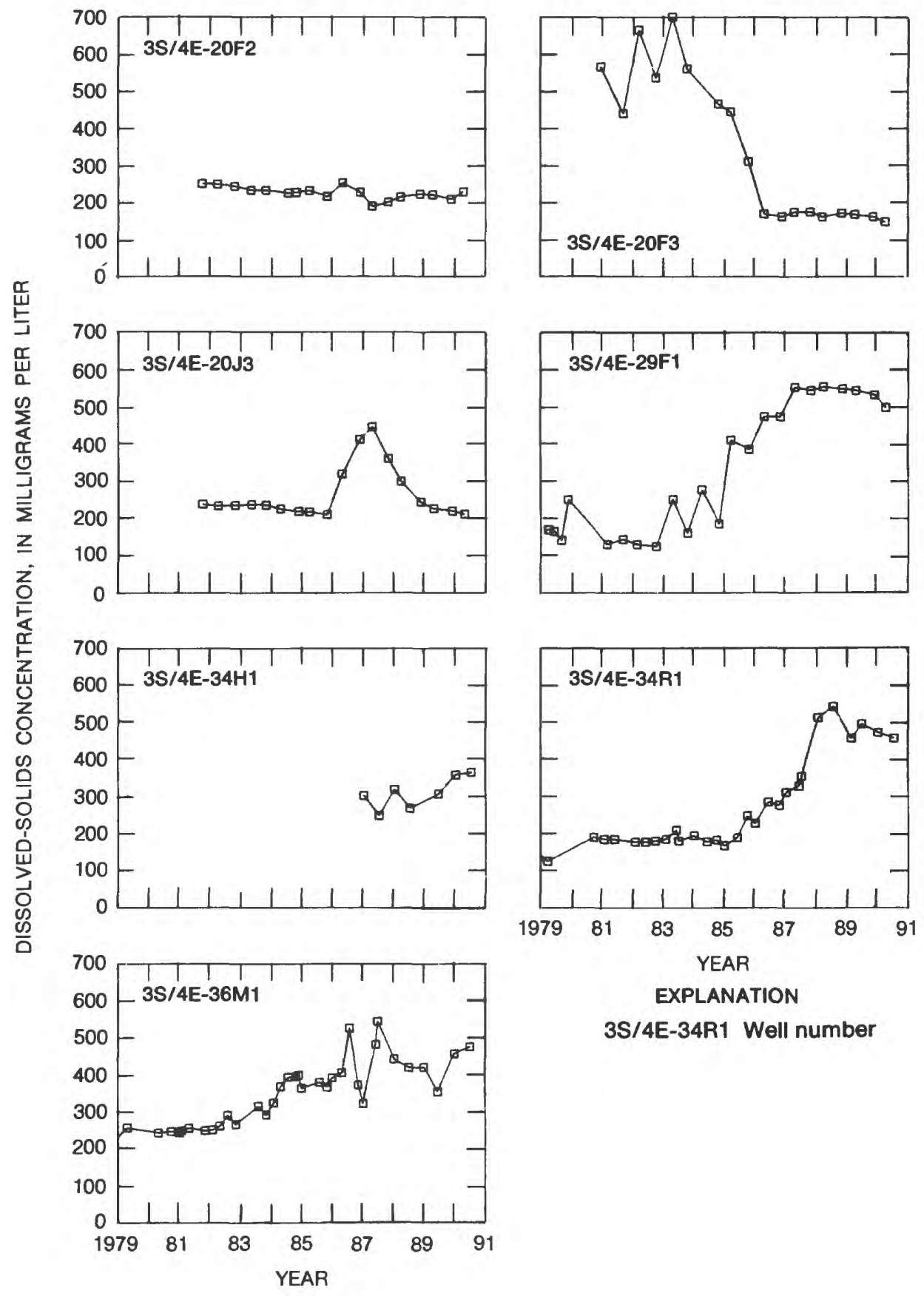

Figure 6. Continued. 


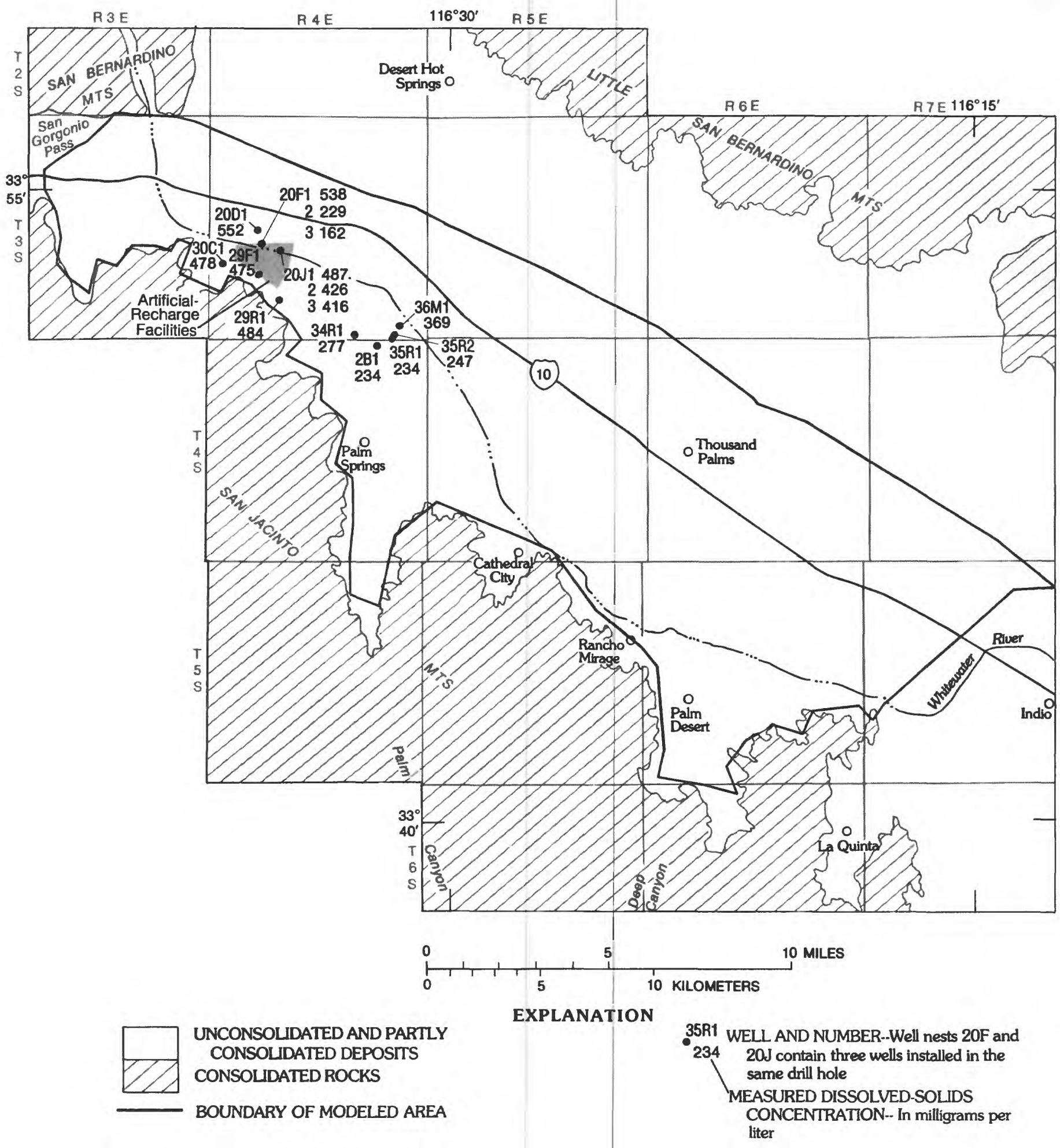

Figure 7. Dissolved-solids concentration in water from selected wells near the artificial-recharge area, autumn 1986. 


\section{EVALUATION OF EXISTING MODEL}

\section{Description of Model}

Swain (1978) developed two-dimensional finite-element models of ground-water flow and transport for the upper Coachella Valley. The flow model was based on an analog model of the same area (Tyley, 1974). An outline of the area modeled is shown in figure 1 . The flow model consisted of 209 isoparametric quadrilateral elements ranging in area from 0.14 to 3.6 $\mathrm{mi}^{2}$. Swain (1978) completed steady-state and transient calibrations of the flow model. In the steady-state calibration, recharge and transmissivity values were adjusted until simulated water levels reasonably matched measured water levels in 1936, when hydrologic conditions were assumed to be in equilibrium. In the transient calibration, storage-coefficient values were adjusted until simulated water levels reasonably matched measured water levels for the period 1936-68. The flow model was further tested by comparing simulated and measured water levels for the period 1968-74. A finer mesh (312 elements, with areas ranging from 0.015 to $0.2 \mathrm{mi}^{2}$ ) was used to model solute transport in the artificial-recharge area. Temporal water-quality data were not available for use in calibrating the transport model.

Swain used his flow and transport models to evaluate the potential effects of a proposed artificial-recharge program. Water levels and dissolved-solids concentrations were simulated using estimated rates of artificial recharge and pumpage for three time periods: 1974-80, 1981-90, and 1991-2000.

In the discussion that follows, Swain's (1978) flow model, hereafter referred to as "the existing model," is evaluated for the purposes of determining the benefits of additional modeling effort and data collection. Some changes to the existing model were necessary for the analysis; however, constructing a new model was not an objective of the study.

\section{Conversion of Existing Model to the Computer Code SUTRA}

The first step in evaluating the existing model (Swain, 1978) was to retrieve the original computer program and input files and recreate some of the original simulations. Second, in the interest of utilizing a well-documented model in current (1991) common use, the original input files were converted for use with SUTRA (Voss, 1984), a two-dimensional areal simulation computer code for ground-water flow and solute transport developed by the U.S. Geological Survey.

The mesh used for the SUTRA model, shown in figure 8, contains 255 nodes and 209 elements. The mesh is exactly the same as that used in the existing model except that nodes located on the sides of elements were eliminated. Pumpage and recharge rates assigned to side nodes in the existing model were distributed to surrounding nodes in SUTRA. Because SUTRA assigns storage properties and thickness by node and the existing model assigned them by element, the nodal values for these parameters in SUTRA were estimated as the mean of the elemental values assigned in the existing model. No other changes were required, other than renumbering of nodes.

As written, SUTRA deals with specific storage, which is a function of matrix (rock) compressibility, fluid compressibility, and porosity. Of these terms, only porosity is allowed to vary spatially in the code. In the Coachella Valley, it is necessary to simulate spatially varying aquifer storage properties. In addition, when solving the two-dimensional ground-water flow equation for a confined aquifer it is useful to input the aquifer storage coefficient directly rather than compute it as a function of its components. To address these two needs, SUTRA was modified to allow an aquifer storage coefficient to be directly input for each node. The storagecoefficient values then are converted to specific 

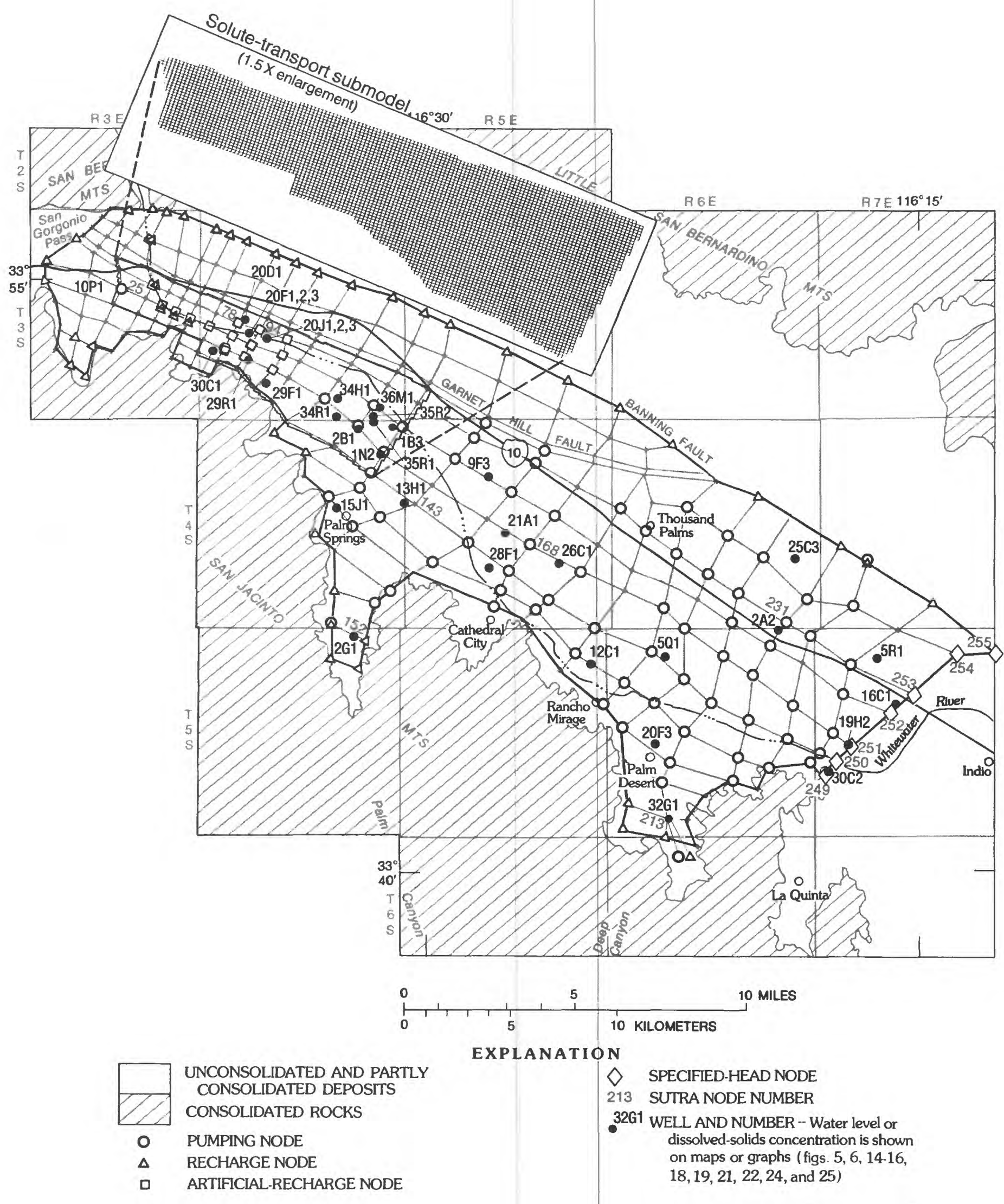

Figure 8. Model mesh, source/sink nodes, boundary conditions, and monitoring-well locations for the SUTRA model, and location of solute-transport submodel. 
storage by dividing the storage coefficients by aquifer thickness. The specific changes made to the SUTRA code are discussed in appendix 6.

\section{Hydrogeologic Parameters Used in Existing Model}

Tyley (1974) established a preliminary transmissivity distribution for the upper Coachella Valley on the basis of drillers' logs and specificcapacity tests. These preliminary values then were revised and separated into aquifer saturated thickness and hydraulic conductivity as part of the steady-state model calibrations of both Tyley (1974) and Swain (1978). These values are shown in figures 9 and 10 . The hydraulic-conductivity values were not modified in this study. As is discussed below, the saturated thickness values in the artificialrecharge area were increased to reflect the effects of rising water levels.

Tyley (1974) derived a preliminary distribution for storage coefficient that was based on a qualitative analysis of the depositional system of the valley. Swain (1978) modified this distribution in the course of transient-model calibration. The storage-coefficient distribution used by Swain (1978) is shown in figure 11. As is discussed below, this distribution was modified in the modeling for this study.

\section{Reproduction, Using SUTRA, of Previous Existing-Model Simulations}

To test the success of the conversion of the existing two-dimensional flow model to a twodimensional SUTRA code, several of the scenarios described by Swain (1978) were simulated. A plot of steady-state (1936) water levels simulated by SUTRA is shown in figure 12 . The discontinuity of the water-level contours reflects the barrier effect of the Garnet Hill fault. These results closely match the map of 1936 water levels (assumed to represent steady-state conditions) presented by Tyley (1974, fig. 9). Simulated water-level changes for 1974-80, with artificial recharge, as simulated by SUTRA (fig. 13), closely match those of Swain (1978, fig. 19).

\section{New Transient Simulations for 1979-86}

After the existing model was converted to SUTRA and tested, the model was used to simulate the period $1979-86$. In the 1980 's, the volume of artificial recharge greatly increased (see table 1 and fig. 4). Applying the SUTRA model to this more recent time period provides a means for testing several assumptions implicit in the existing model and for improving understanding of the geohydrologic system in the upper valley.

The following changes were made to the existing-model inputs. The 1979-86 net pumpage and artificial-recharge values were input into the SUTRA model. Twenty percent of the water diverted from the Colorado River Aqueduct (see table 1), up to a maximum of $50 \mathrm{ft}^{3} / \mathrm{s}$, was assumed to recharge in the Whitewater River channel upstream of the recharge facilities. On the basis of the area of the artificialrecharge facilities (700 acres), and an estimated evaporation rate of 66 to $72 \mathrm{in} / \mathrm{yr}$ (see Hely and Peck, 1964, pl. 6), 4,000 acre-ft/yr of the water diverted to the recharge facilities was assumed to evaporate. This value probably is an overestimate for evaporation in the years 197984 , when the recharge facilities covered a smaller area and when most recharge took place during cooler, winter months. Artificial recharge is input as a source term lagged 3 months from the beginning of the year. This lag period was chosen on the basis of an analysis of hydrographs of wells in the artificial-recharge area. Initially, the only other change made to the existing model was to modify the southeastern boundary conditions. Because water levels are slowly declining in that part of the valley, the constant-head boundary used in the existing 

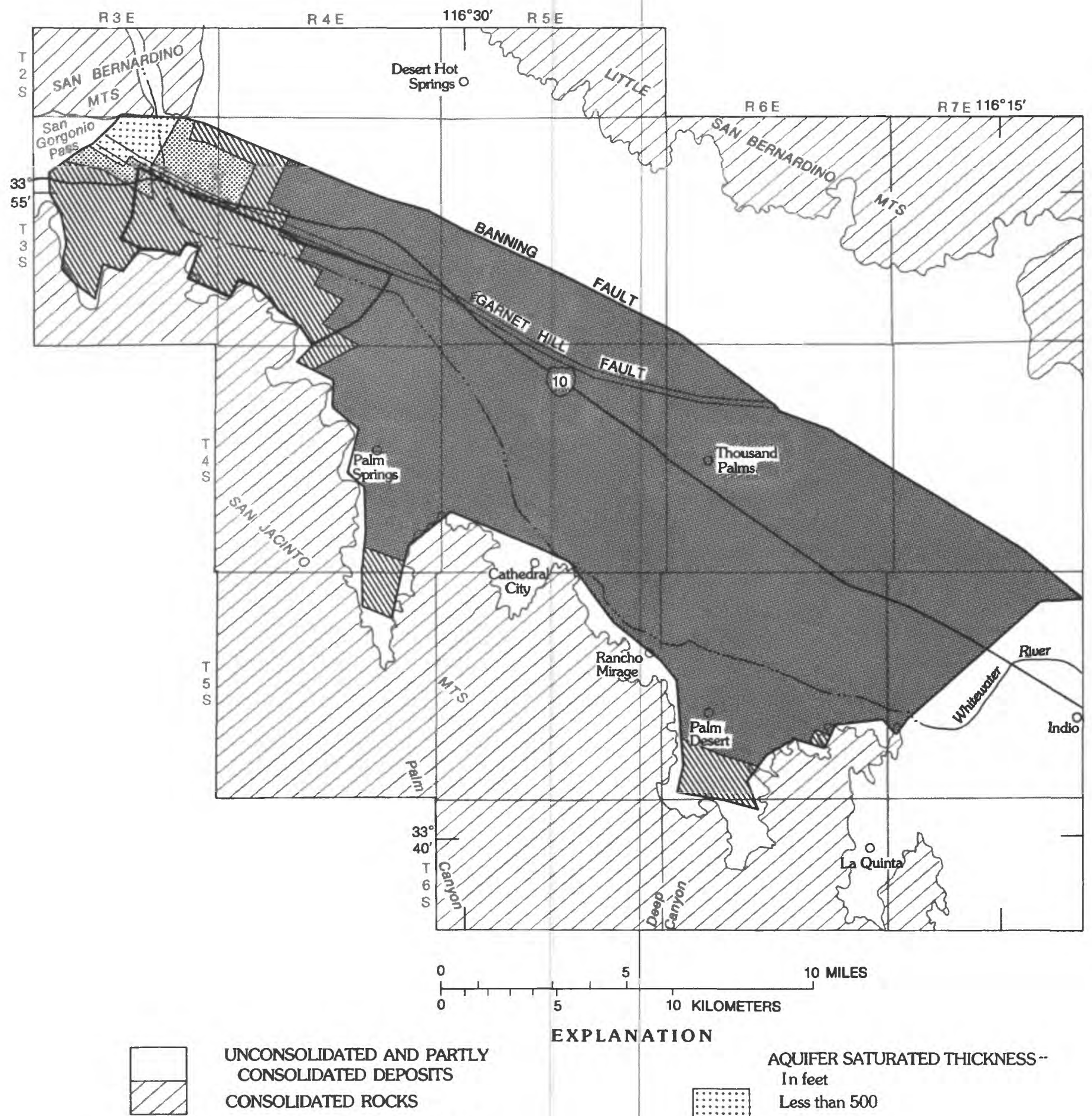

UNCONSOLIDATED AND PARTLY CONSOLIDATED DEPOSTTS CONSOLIDATED ROCKS

BOUNDARY OF MODELED AREA

BOUNDARY OF AREA WITHIN WHICH SATURATED THICKNESS WAS INCREASED TO ACCOUNT FOR RISE IN WATER LEVELS

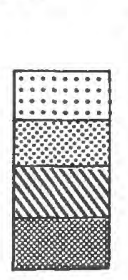

AQUIFER SATURATED THICKNESS-In feet

Less than 500

500 to 700

700 to 900

900 to 1,000

Figure 9. Aquifer saturated thickness in the model area. 

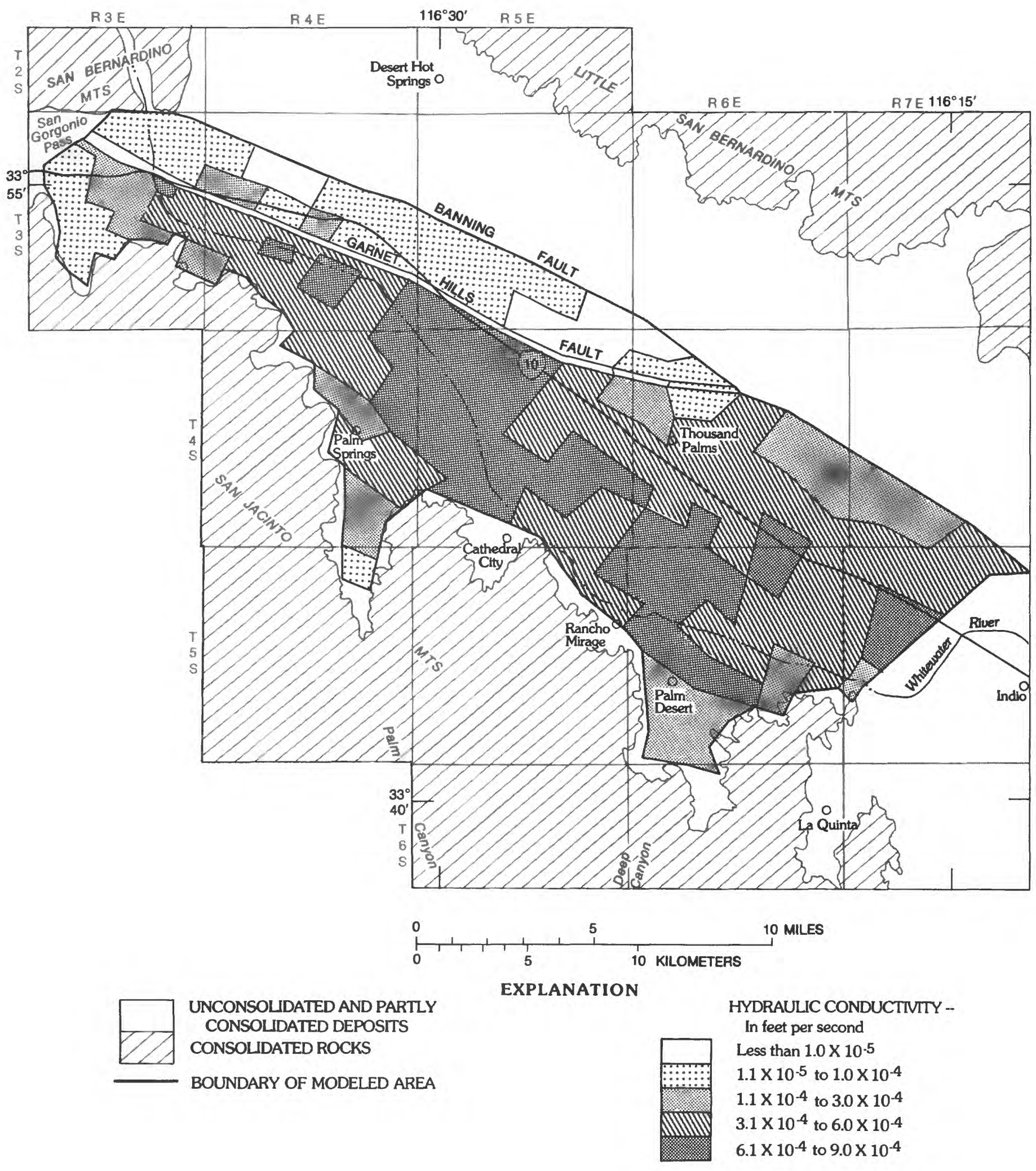

Figure 10. Hydraulic conductivity in the model area. 


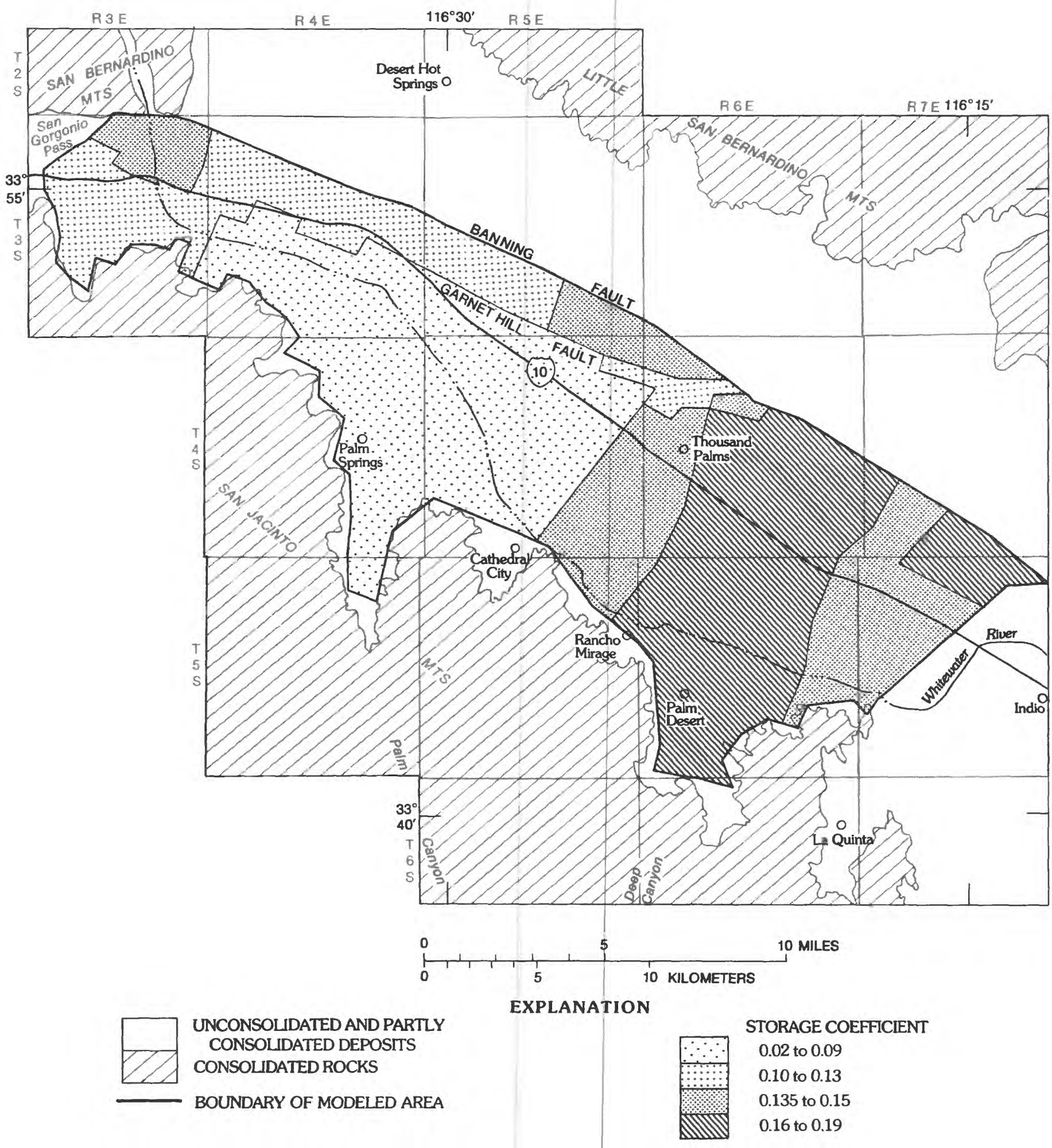

Figure 11. Storage-coefficient distribution used in existing model. 

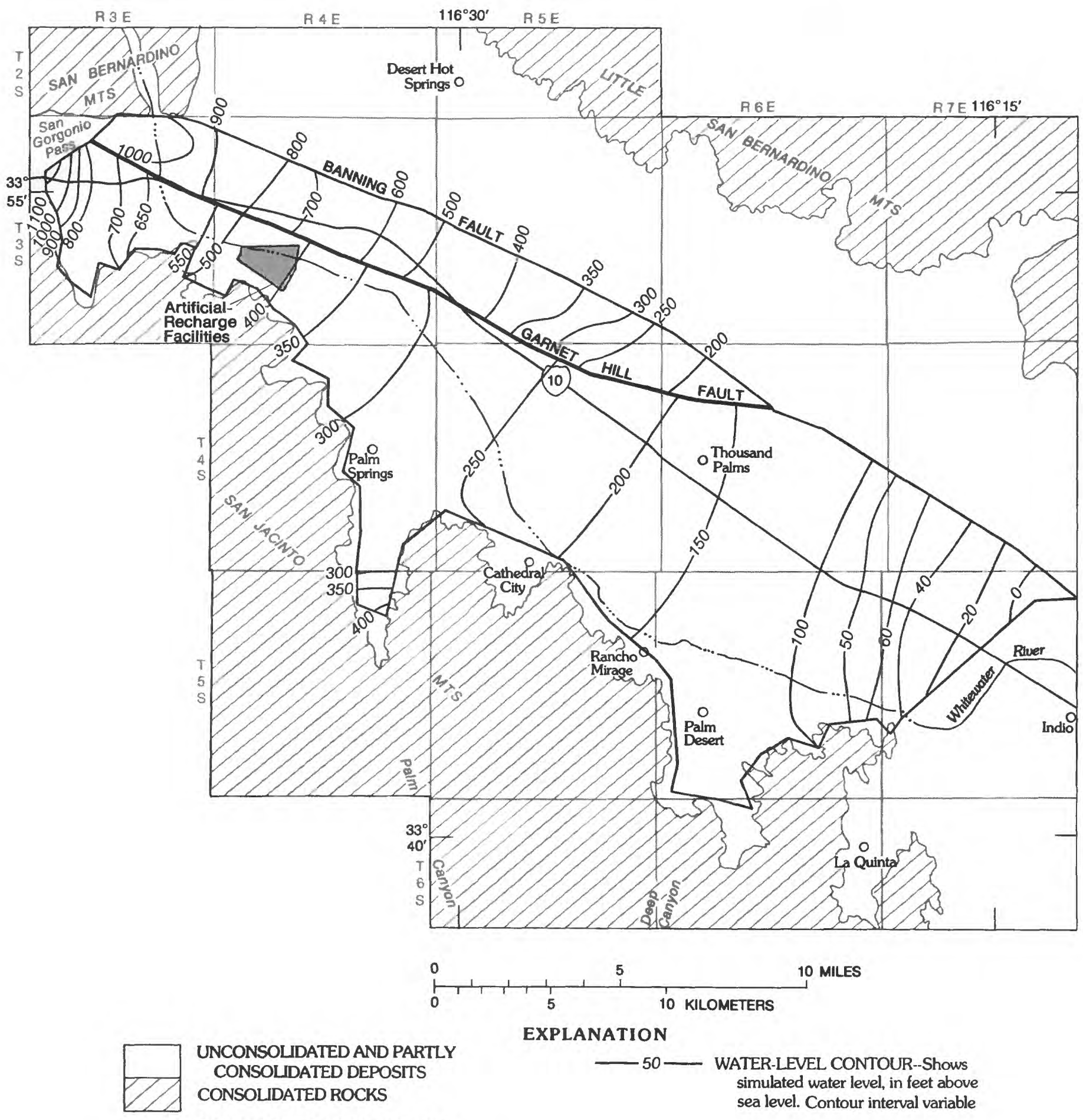

EXPLANATION

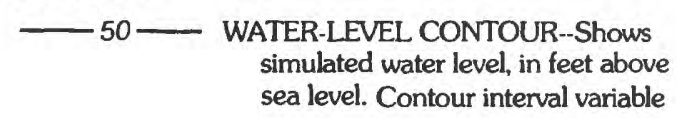

BOUNDARY OF MODELED AREA

Figure 12. Simulated steady-state (1936) water levels using SUTRA model. 


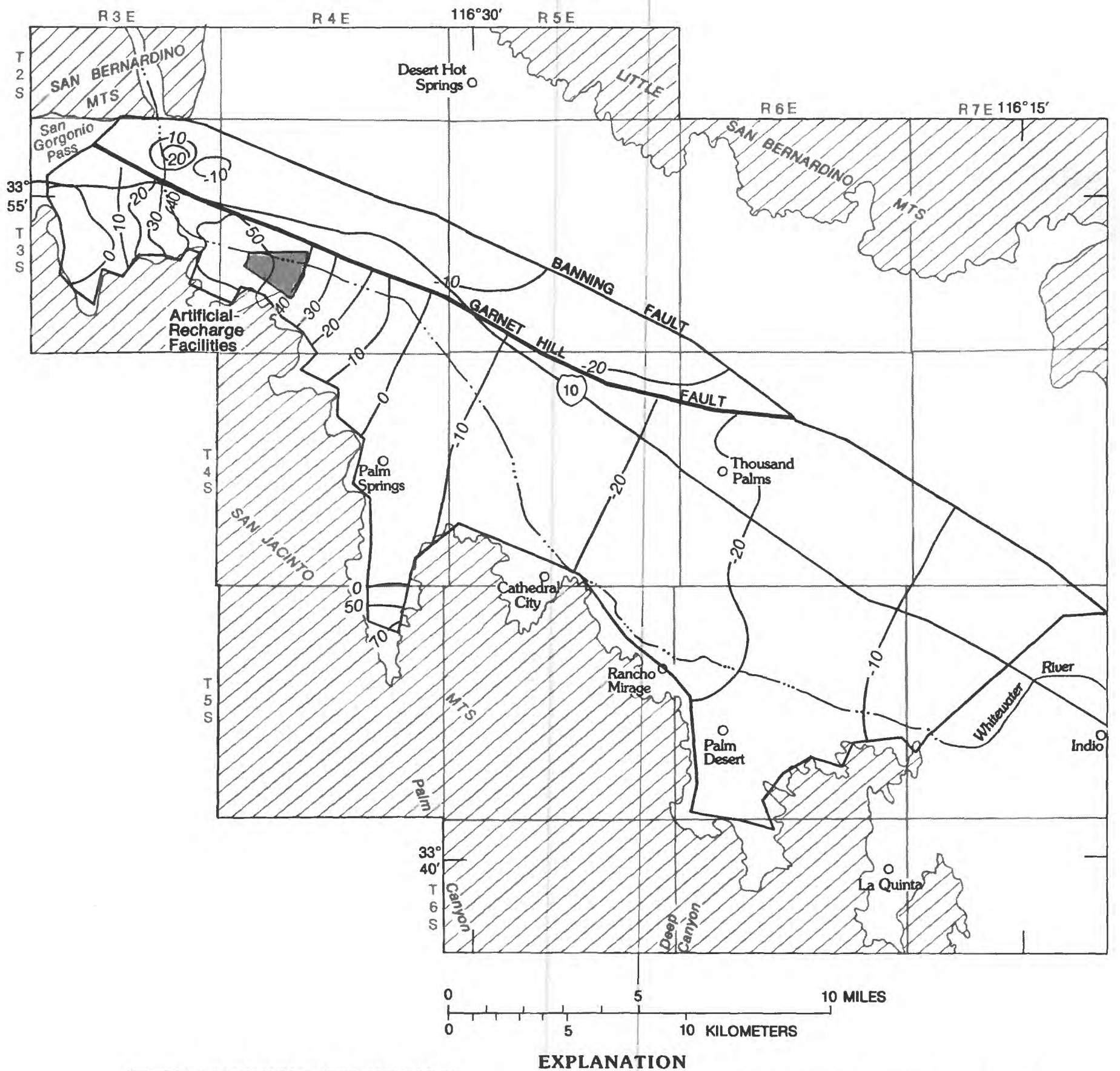

UNCONSOLIDATED AND PARTLY
CONSOLIDATED DEPOSITS
CONSOLIDATED ROCKS

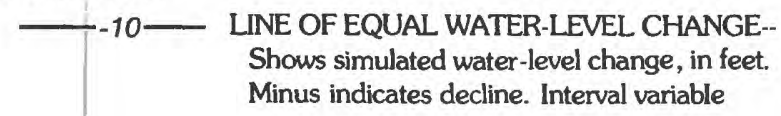

BOUNDARY OF MODELED AREA

Figure 13. Simulated water-level change, 1974-80, using SUTRA model. 
model is no longer valid, and it was replaced by a specified-head boundary. The heads specified for each year of the 8-year simulation period for the six nodes at the southeast end of the model are given in appendix 7. These heads were based on water levels measured at wells 5S/7E-16C1, $-19 \mathrm{H} 2$, and $-30 \mathrm{C} 2$ (see fig. 5). The initial head conditions for the 1979-86 simulation were generated from the 1974-80 SUTRA simulation with artificial recharge described in the previous section. The SUTRA "unit 55" input file containing these initial head values is shown in appendix 8.

For the 1979-86 simulation, simulated and measured water levels for selected wells are shown in figure 14. Simulated water levels for three wells, 3S/3E-10P1, 5S/4E-2G1, and $5 S / 6 E-32 G 1$, are less than measured water levels for the entire model period. The reasons for this underestimation are discussed below. The simulated water levels for other wells correlate reasonably well with measured water levels for the period 1979-84. The simulated rise in water levels in 1985 and 1986, however, is significantly larger than the measured rise (see wells 3S/4E-20D1 and-20F1, for example). There are three possible factors that may contribute to this divergence between model simulations and measured values: Transmissivity is underestimated, artificial-recharge rates are overestimated, or the storage coefficient is underestimated.

The 300- to 400-foot rise in water levels in the artificial-recharge area in 1985 and 1986 is a significant fraction of the assumed initial saturated thickness of the aquifer (see fig. 9). SUTRA, when used areally, cannot simulate conditions of varying saturated thickness. For this study, a simplified method of incorporating the effects of increased saturated thickness was employed. The saturated thickness for all model nodes in the artificial-recharge area was increased $350 \mathrm{ft}$ from values previously used in the model (see fig. 9). Clearly this overestimates saturated thickness prior to 1985; however, it grossly approximates the increased transmissivity during the years with the highest recharge rates. Simulated and measured 197986 water levels for the same wells when the effects of increased transmissivity (an increase in saturated thickness) are considered are shown in figure 15 . The simulated water-level rise in the artificial-recharge area in 1985 and 1986 still is much larger than the measured rise. Water levels are relatively insensitive to transmissivity values in this area.

Model simulations indicate that assumed artificial-recharge rates would have to be reduced to 150,000 acre-ft/yr in 1985 and 1986 to produce simulated water levels that reasonably match measured levels (see fig. 16), This implies recharge rates that are 60,000 to 100,000 acre-ft/yr lower than the rates given in table 1. However, it is unlikely that the artificial-recharge rates in table 1 are greatly overestimated. The amount of Colorado River water released into the Whitewater River is gaged, and local officials report that no water was "wasted" downstream of the ponds. Although evaporation rates are uncertain, it is not likely that 1985-86 evaporation was significantly higher than the 4,000 acre- $\mathrm{ft} / \mathrm{yr}$ value used in the model.

The average infiltration rates implied by the assumed artificial-recharge quantities given in table 1 are consistent with sparse available data. For example, the recharge of 258,000 acre-ft over a 700-acre area in 1986 implies an average infiltration rate of about $1 \mathrm{ft} / \mathrm{d}$. This is consistent with recent data collected by the Coachella Valley Water District (Levy, 1988) that indicate that the infiltration rate at a recharge pond declines from an initial value of about $20 \mathrm{ft} / \mathrm{d}$ to a long-term value of about $0.6 \mathrm{ft} / \mathrm{d}$.

Much of the difference between simulated and measured water levels in 1985 and 1986 in the artificial-recharge area likely is due to the storage-coefficient value of 0.02 assumed in that area (see fig. 11). Storage-coefficient values 


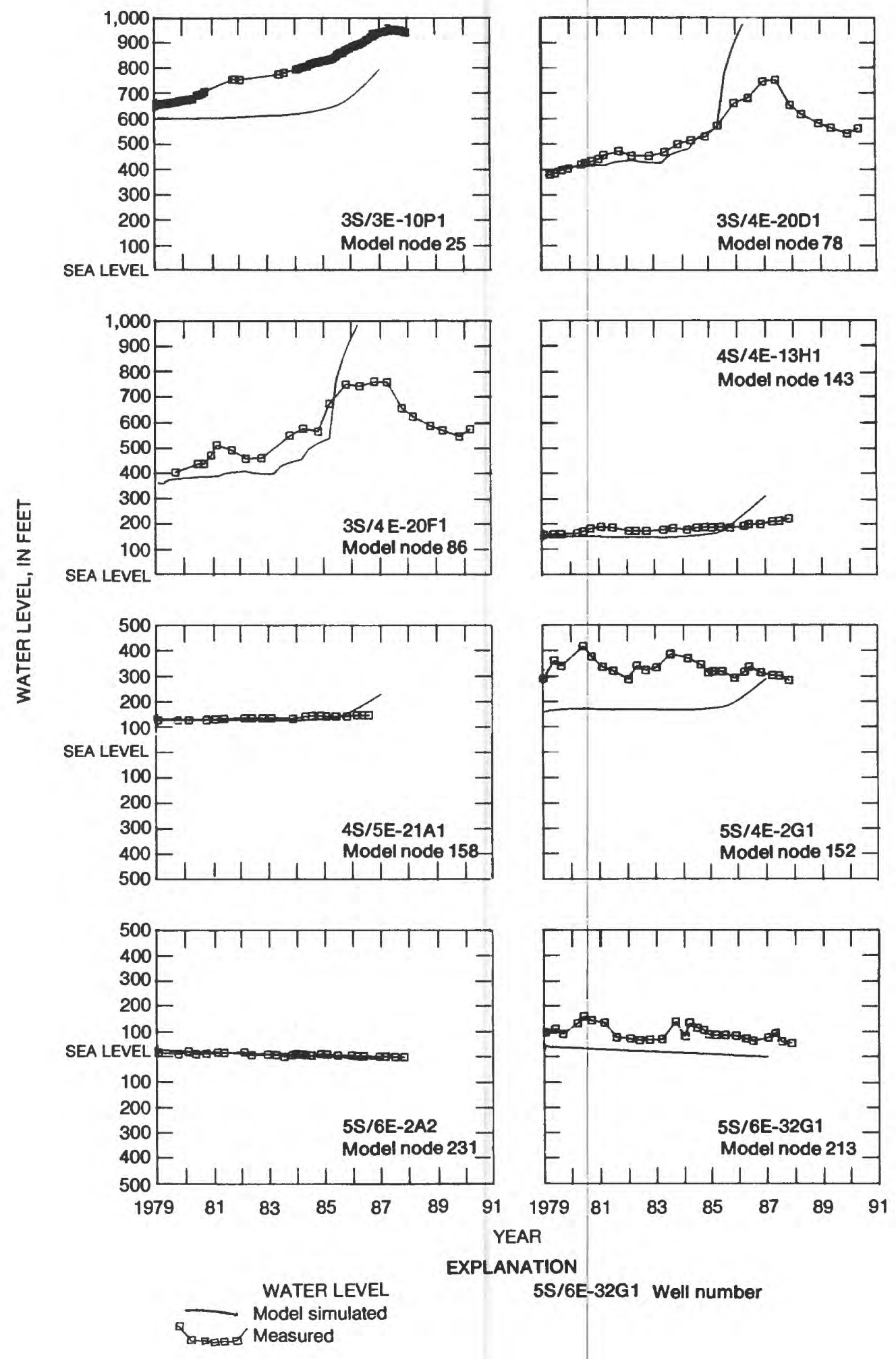

Figure 14. Simulated water levels, 1979-86, using all parameters from existing model, and measured water levels at selected wells. 

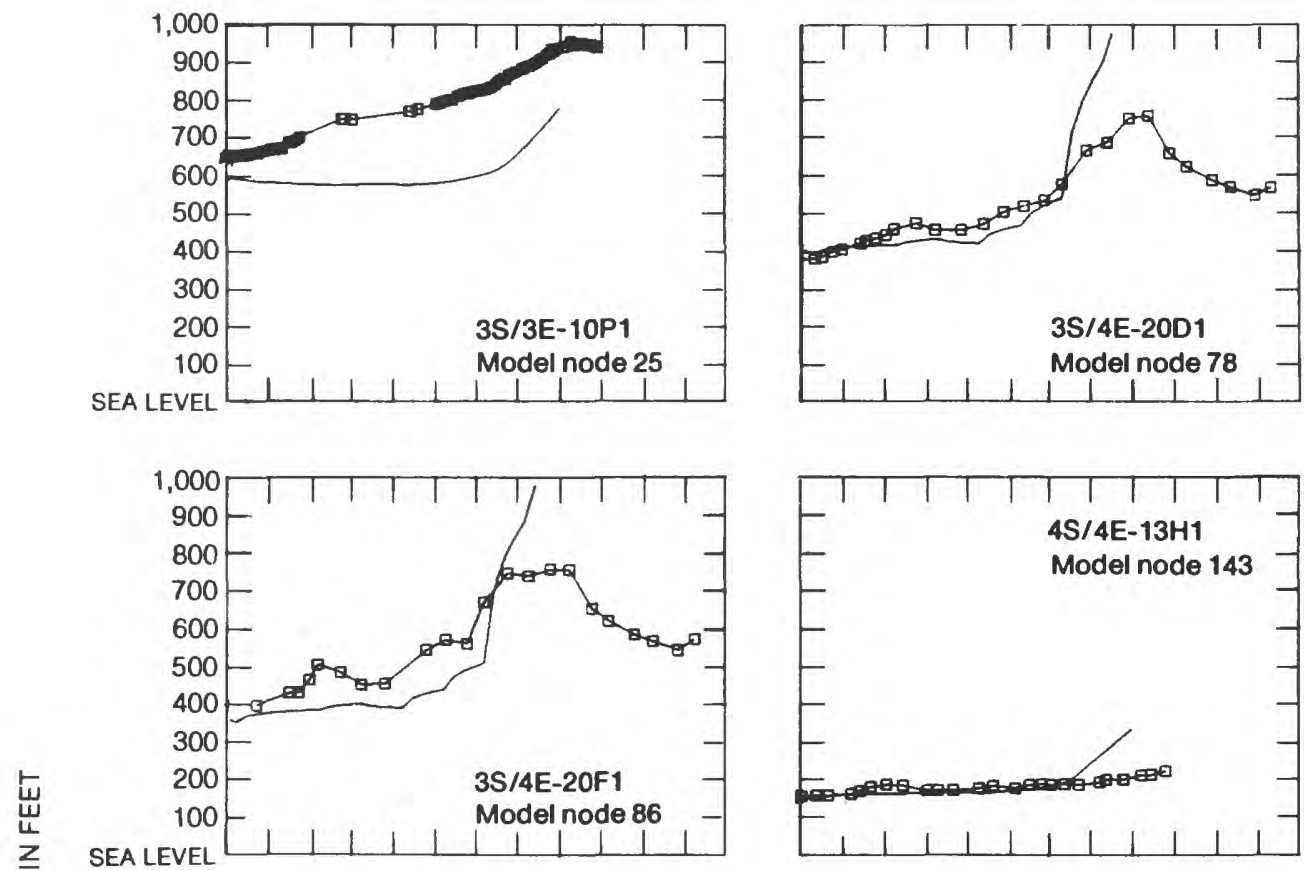

交

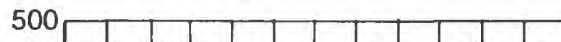
$400-$ $300-$

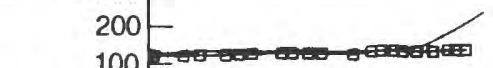
SEA LEVEL $100-$ 200 $300-$
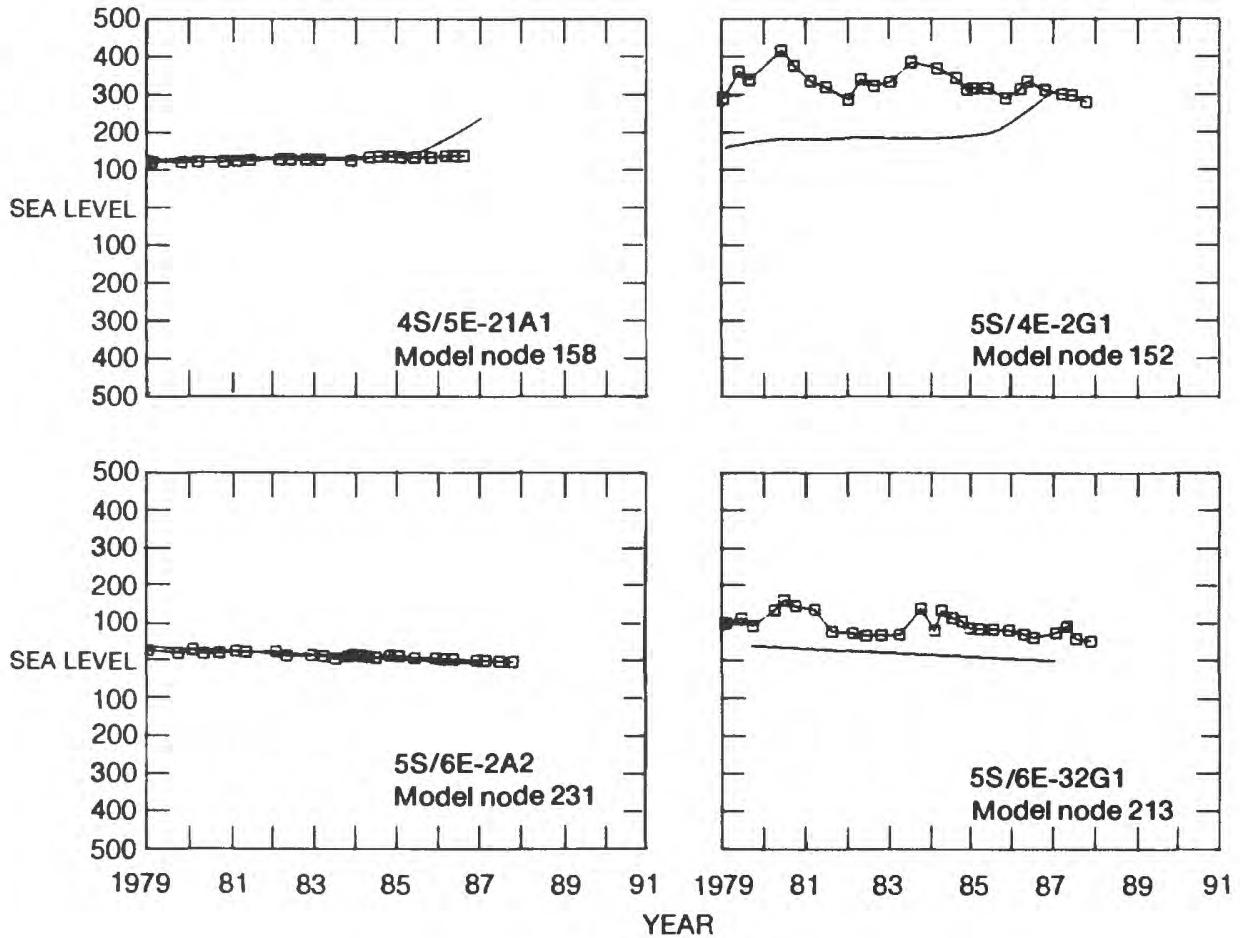

WATER LEVEL

EXPLANATION

Model simulated

5S/6E-32G1 Well number

Measured

Figure 15. Simulated water levels, 1979-86, using all parameters from existing model, except assuming 350-foot increase in saturated thickness of aquifer in the artificial-recharge area, and measured water levels at selected wells. 


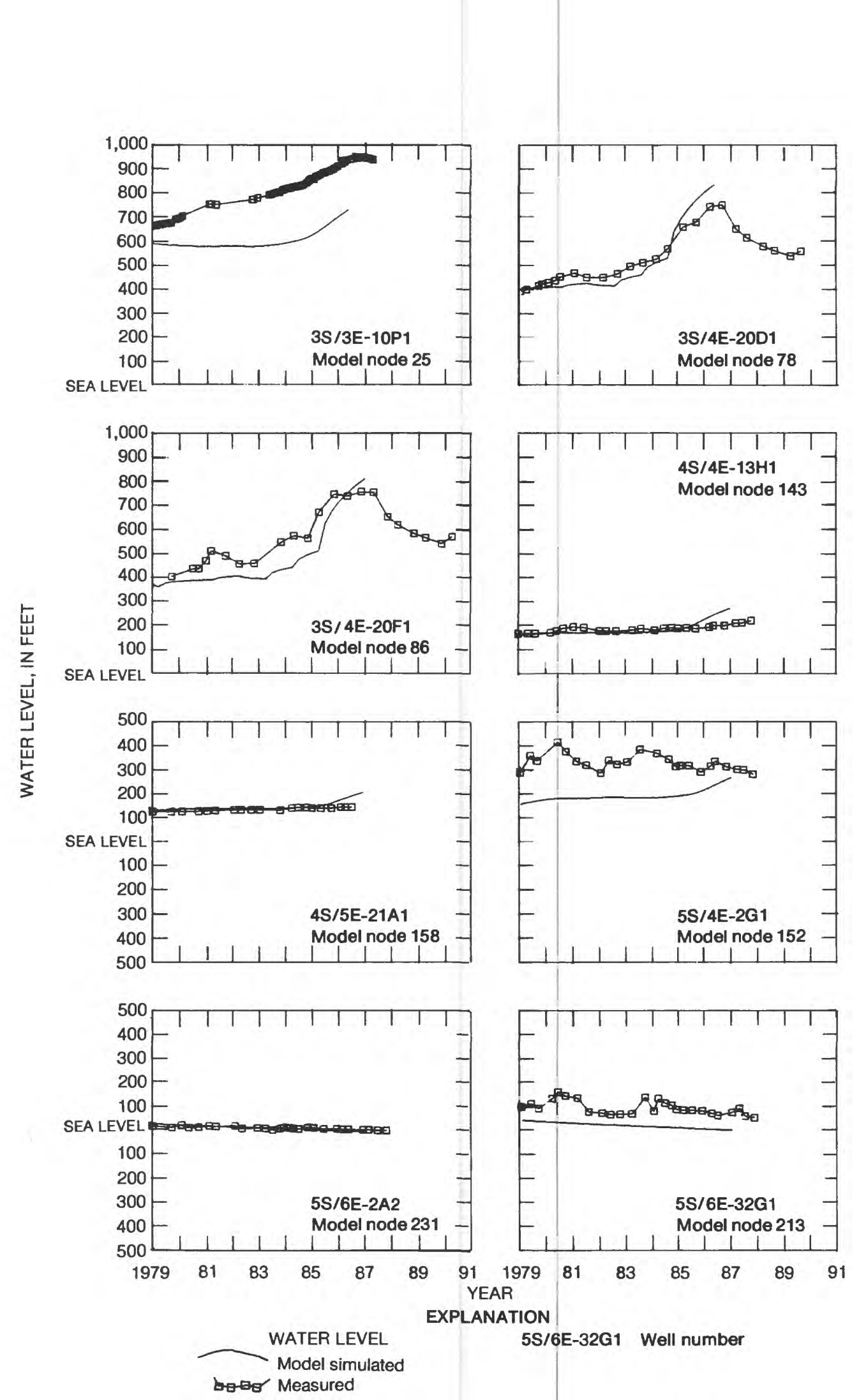

Figure 16. Simulated water levels, 1979-86, using all parameters from existing model, except assuming 350-foot increase in saturated thickness of aquifer in the artificial-recharge area and assuming a maximum of 150,000 acre-feet per year of artificial recharge, and measured water levels at selected wells. 
were adjusted upward in this area to $0.10-0.15$. These adjusted values were more consistent with the values in the rest of the model $(0.10$ $0.19)$. The revised storage-coefficient distribution is shown in figure 17. The SUTRA "unit 5" input file for the 1979-86 simulation using this revised distribution is given in appendix 9. Simulated and measured water levels are shown in figures 18 and 19. The match in the artificialrecharge area for the high-recharge years, 1985 and 1986, is much better than that using the previous storage-coefficient distribution (see fig. $15)$. Note, however, that the previous storagecoefficient distribution yields a slightly better match for the years 1979-84. This illustrates the limitations of modeling a complicated hydrogeologic system (see fig. 2) with a two-dimensional model. During the period of the original model calibration and during the first years of this model application, the water levels in wells in the artificial-recharge area showed a semiconfined response, with a storage-coefficient value of approximately 0.02 . However, the change in hydrologic conditions in 1985 and 1986 caused a significant change in aquifer characteristics. As much as $400 \mathrm{ft}$ of previously unsaturated deposits became saturated. Water levels during that period showed an unconfined response, with storage-coefficient values of 0.10-0.15.

Model simulations carried out for this study end in 1986, because pumpage data were not available for later years. Water levels during the period 1987-89 generally declined because, as described earlier, no artificial recharge occurred from spring 1987 through autumn 1989. When such pumpage data become available, the simulation could be extended to include this period. The match between simulated and measured water-level declines for 1987-89 using the revised storage-coefficient distribution then could be evaluated.

The remainder of this report deals only with model simulations using the revised storagecoefficient distribution, because the main emphasis is on the effects of the large recharge volumes. It is recognized that this distribution yields a poorer match of simulated and measured water levels than the previous storagecoefficient distribution for $1979-84$. As is discussed below, there are several other important disparities between simulated and measured water levels.

As shown in figures 18 and 19, the simulated water levels southeast of San Gorgonio Pass (see wells 3S/3E-10P1 and 3S/4E-20D1 and $-20 \mathrm{~F} 1$ for the period 1979-85) and in Palm and Deep Canyons (see wells 5S/4E-2G1 and 5S/6E-32G1) are too low. This seems to suggest that recharge from streams and underflow from San Gorgonio Pass in the 1980's has been greater than quantities assumed in the studies of Tyley (1974) and Swain (1978) (see table 1). Graphs of cumulative flow and cumulative departure from mean flow for several streams in the Coachella Valley (fig. 20) show that conditions during the mid-1970's to the mid1980 's were considerably wetter than those in the late 1960's and early 1970's when Tyley's and Swain's studies were carried out. Years 1980 and 1983 were particularly wet.

The underestimation of water levels by the model southeast of San Gorgonio Pass suggests that artificial recharge, at least during 1979-85, had a greater effect on water levels in that area than was calculated using the model. This indicates that the spatial distribution of artificial recharge used in the model may be incorrect.

\section{Solute-Transport Simulations for 1979-86}

The effect on water quality of the artificialrecharge water of higher dissolved-solids concentration was simulated with SUTRA using a smaller solute-transport submodel (see fig. 8). The model mesh contains 4,742 nodes and 4,552 elements with dimensions of 300 by 400 $\mathrm{ft}$. The nodes on the northwest and southeast boundaries of the submodel were set as time-varying specified-head boundaries on the 

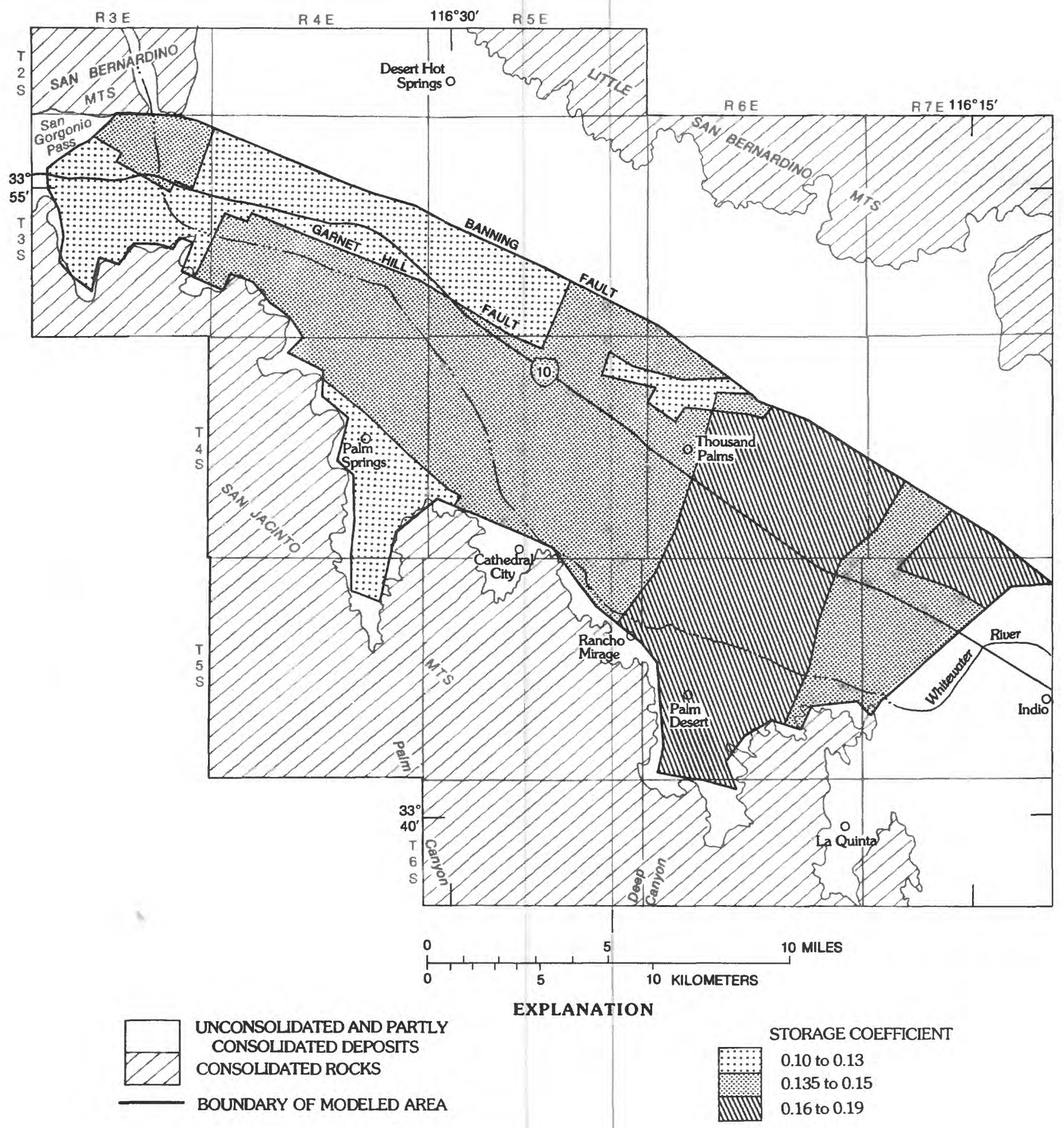

Figure 17. Revised storage-coefficient distribution used in SUTRA model. 

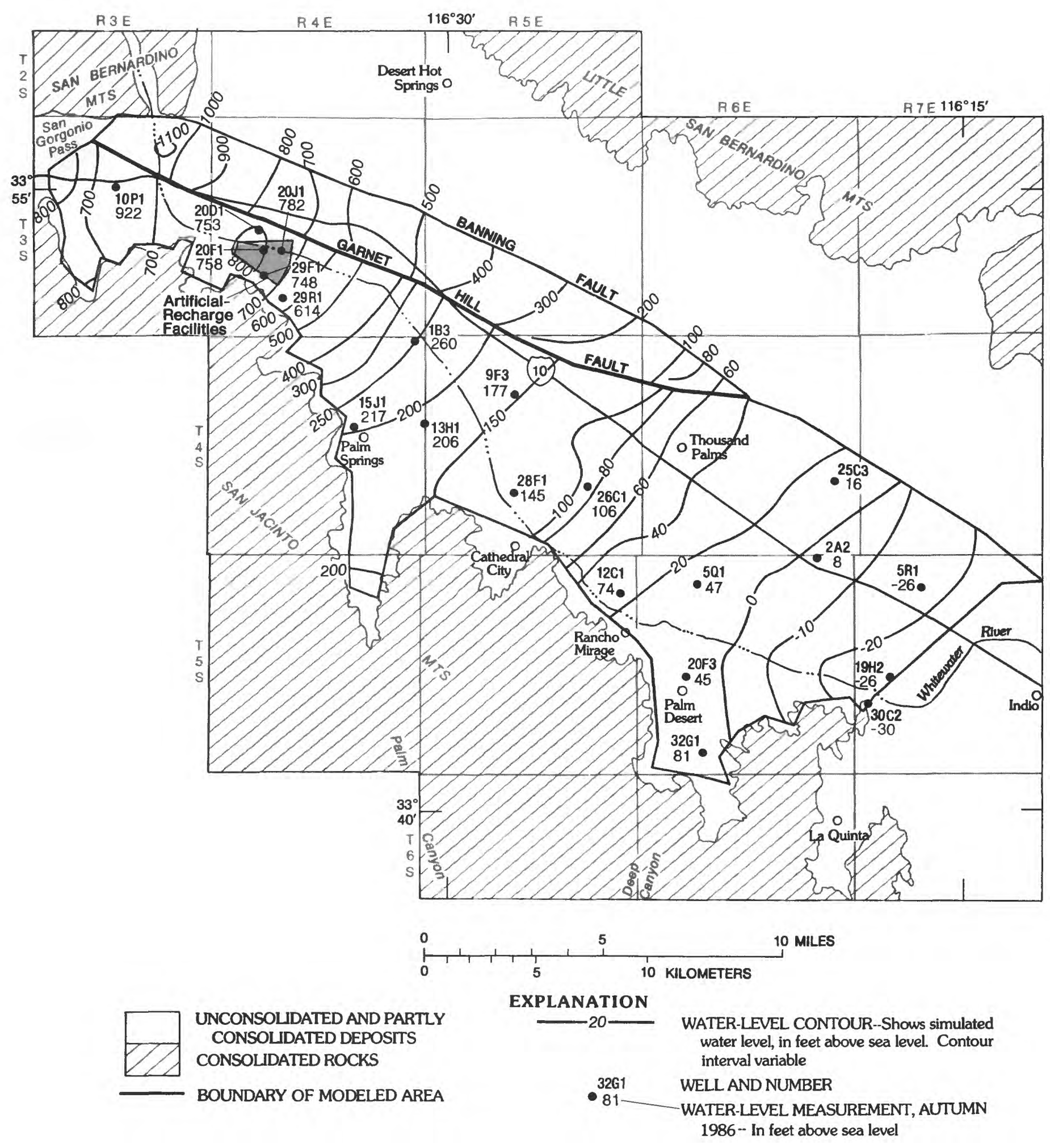

Figure 18. Simulated water levels, with revised storage-coefficient distribution used in SUTRA model, and measured water levels, autumn 1986. 


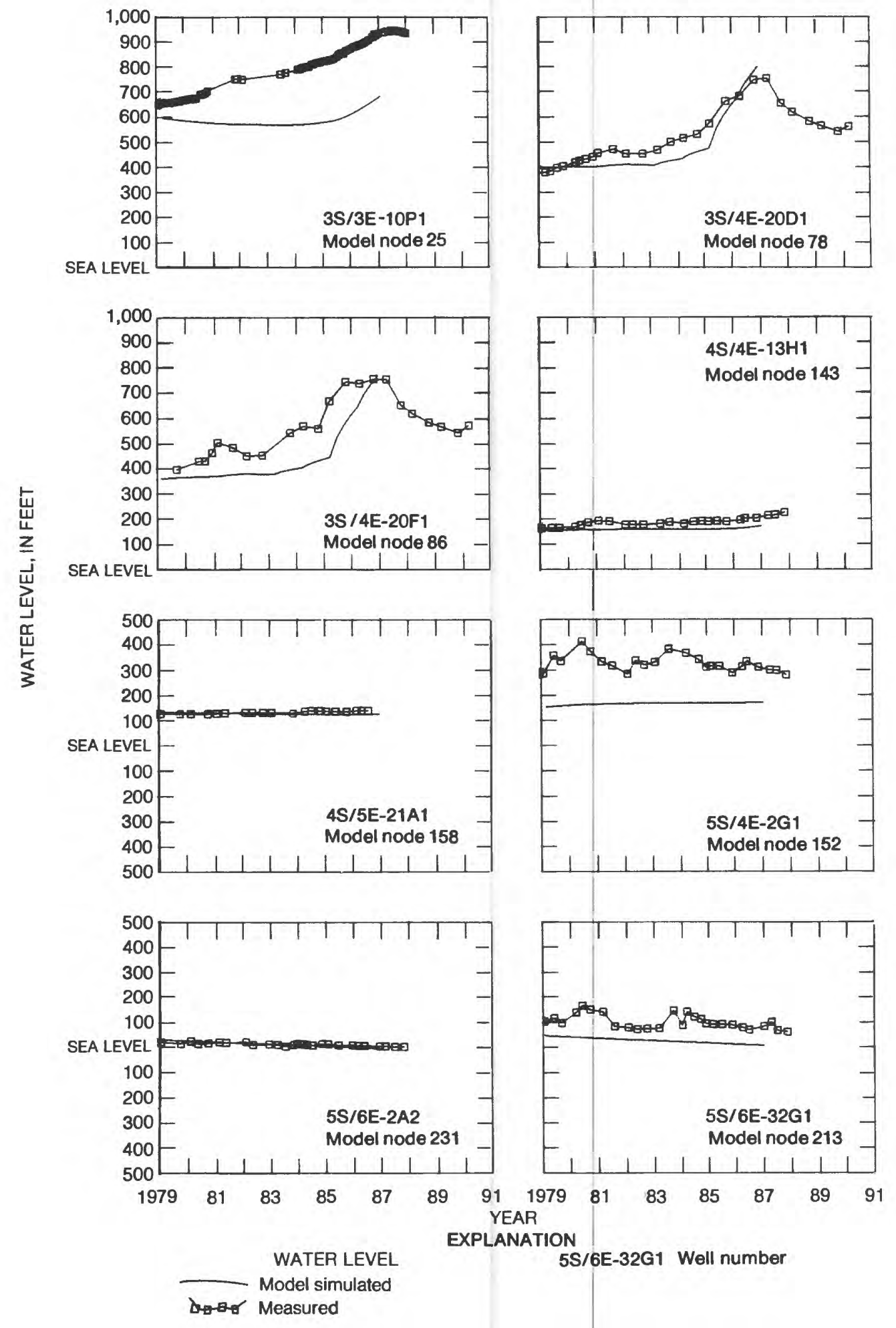

Figure 19. Simulated water levels, 1979-86, with revised storage-coefficient distribution used in SUTRA model, and measured water levels at selected wells. 

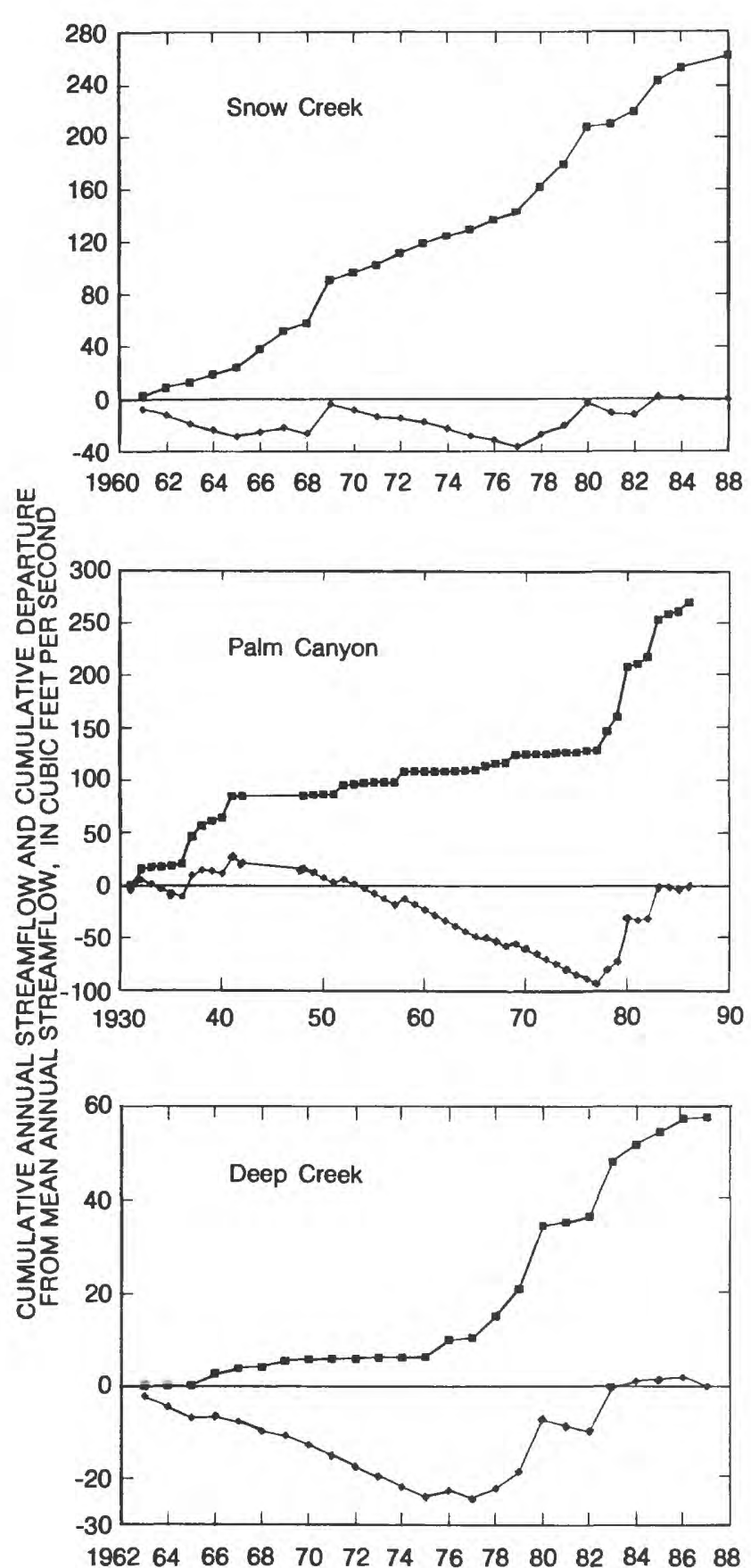

YEAR

EXPLANATION

- cumulative anNual streamflow

- cumulative deParture from mean anNual STREAMFLOW

Figure 20. Cumulative annual flow and cumulative departure from mean annual flow for three Coachella Valley streams. basis of results from the flow model just described. The northeast and southwest boundaries were set as no-flow boundaries. Specifying the northeast boundary, the Banning fault, as a no-flow boundary does not account for the outflow of artificial-recharge water that occurs across the fault. The boundary condition was chosen for reasons of numerical stability in the simulation. Submodel simulations were carried out in which this northeast boundary was set as a time-varying specified-head boundary rather than a no-flow boundary. The concentrations that were produced by these simulations were similar to the concentrations obtained using a no-flow boundary, except that numerical instabilities led to unreasonable computed concentration values near that boundary. The parameter values and source-sink rates from the flow model were used. Stresses from the flow model had to be distributed among nodes representing the same area in the submodel. Assuming no chemical reactions, SUTRA can be used to solve the two-dimensional advection dispersion equation (see Konikow and Bredehoeft, 1978, p. 5-6). Swain's (1978) values for porosity (0.25), longitudinal dispersivity $(100 \mathrm{ft})$, and transverse dispersivity (33 ft) were used.

The solute-transport submodel was used to simulate the increase in dissolved solids resulting from artificial recharge during 1979-86. Initial dissolved-solids concentration in ground water was assumed to be $200 \mathrm{mg} / \mathrm{L}$. Simulated and measured dissolved-solids concentrations at selected wells are shown in figures 21 and 22 . The perforated intervals of these wells are shown in figure 23 superimposed on geologic section $B-B^{\prime}$ (line of section is shown in fig. 21).

As shown in figures 21 and 22 , the model poorly simulates changes in dissolved-solids concentration. The high-dissolved-solids recharge water is moving much faster than is simulated by the model. For example, dissolvedsolids concentration clearly increased in wells 


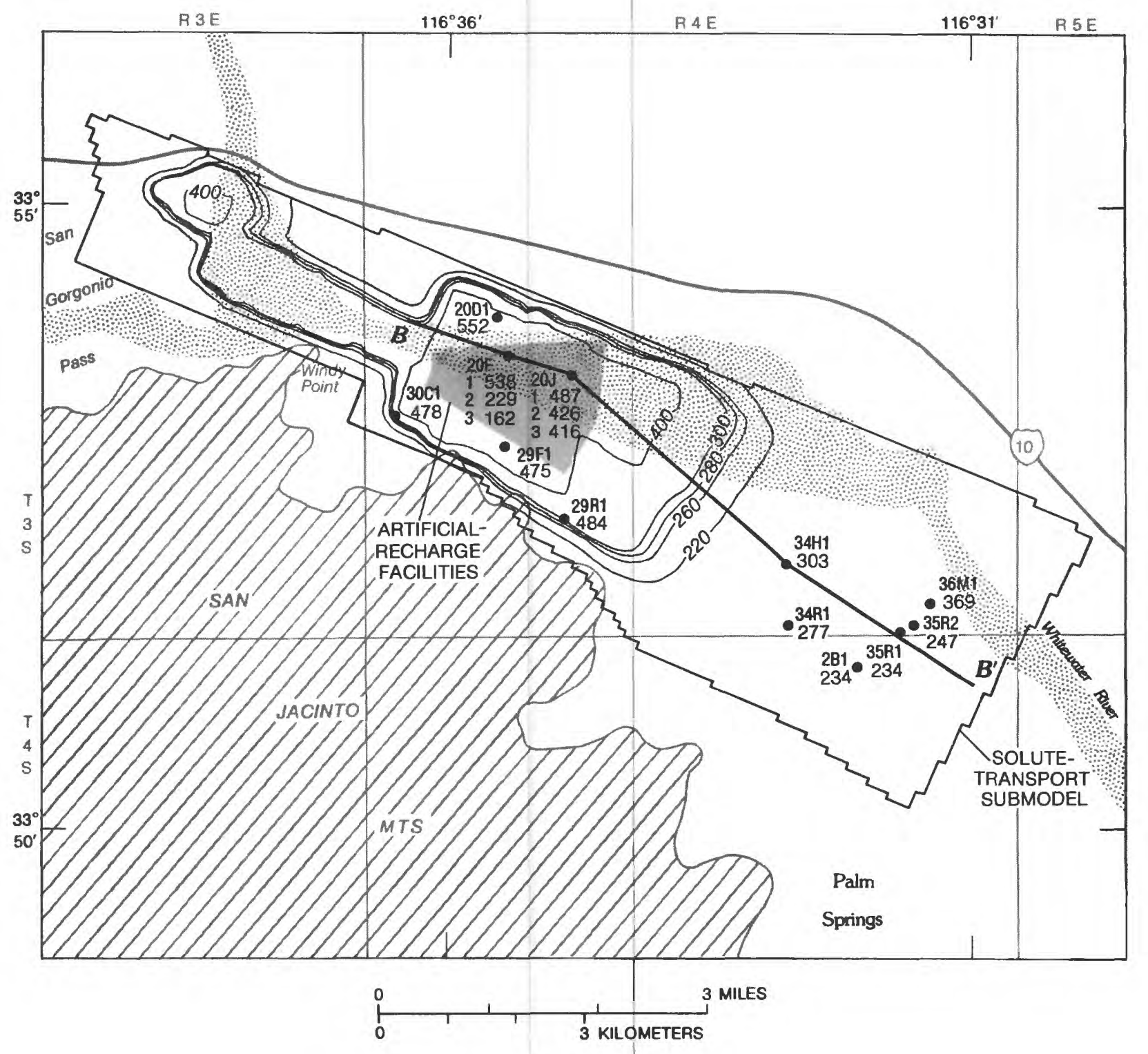

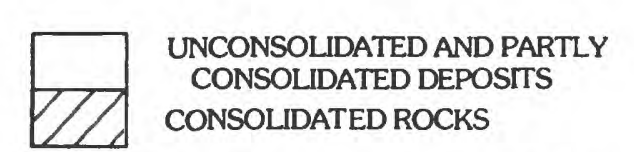

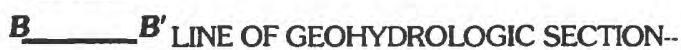

Shown in figure 23
EXPLANATION

Figure 21. Simulated and measured dissolved-solids concentration, autumn 1986, for base-case simulation using the solute-transport submodel. 

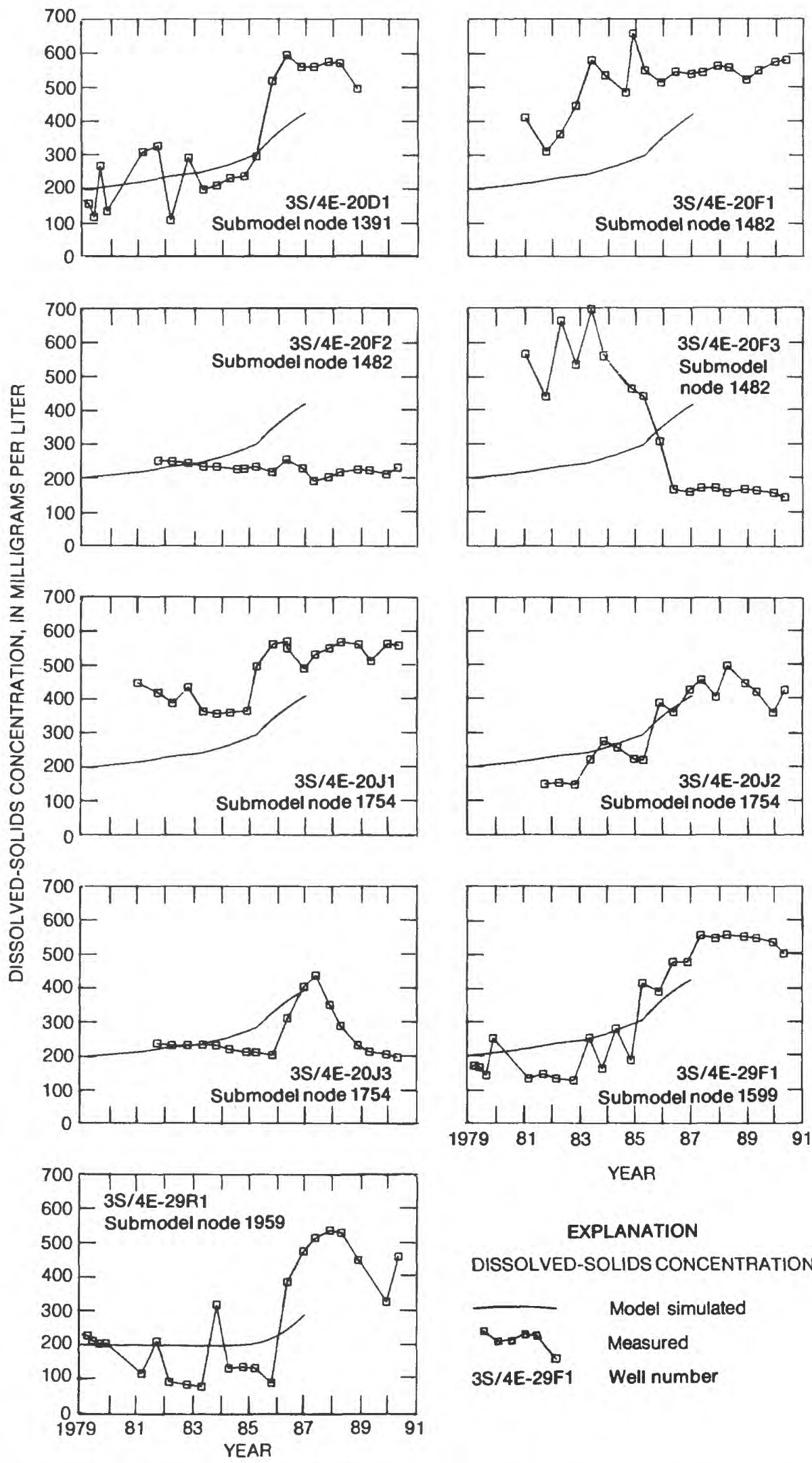

EXPLANATION

DISSOLVED-SOLIDS CONCENTRATION

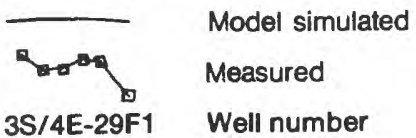

Figure 22. Simulated dissolved-solids concentration, 1979-86, for base-case simulation, and measured dissolved-solids concentration in water from selected wells--Continued on page 34. 

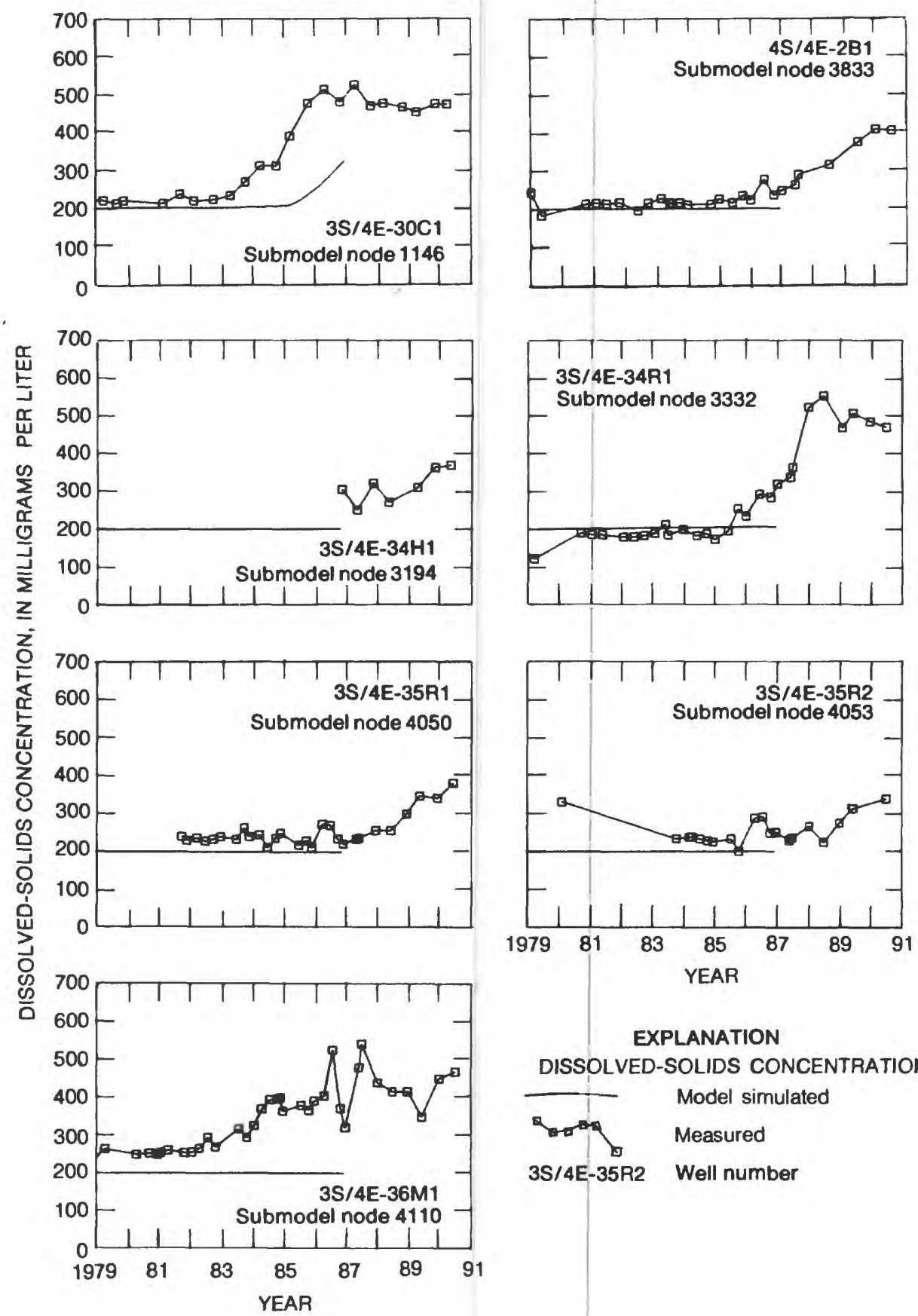

EXPLANATION

DISSOLVED-SOLIDS CONCENTRATION

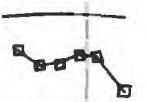

Model simulated

Measured

3S/4E-35R2

Well number

Figure 22. Simulated dissolved-solids concentration, 1979-86, for base-case simulation, and measured dissolved-solids concentration in water from selected wells--Continued. 


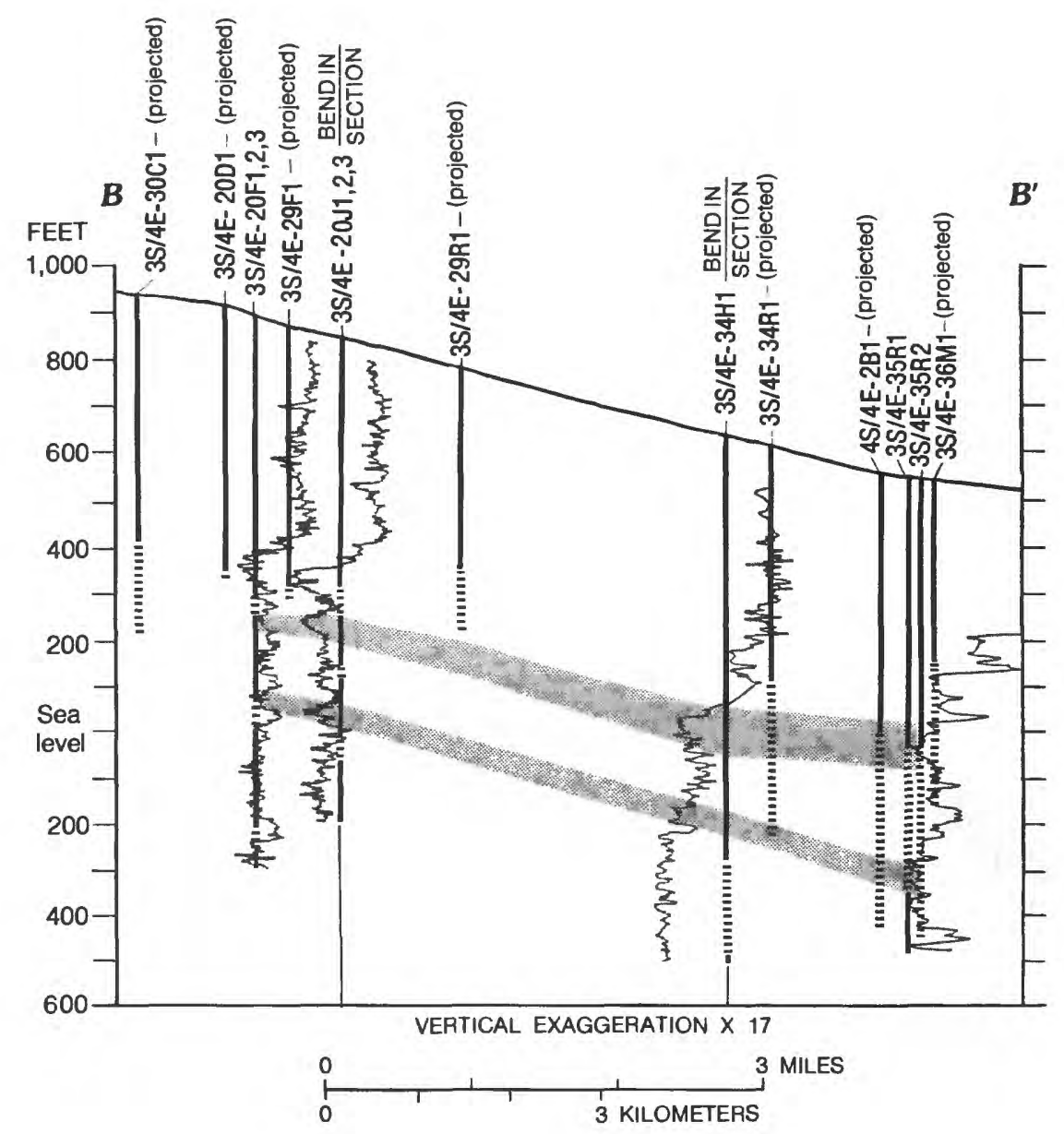

EXPLANATION

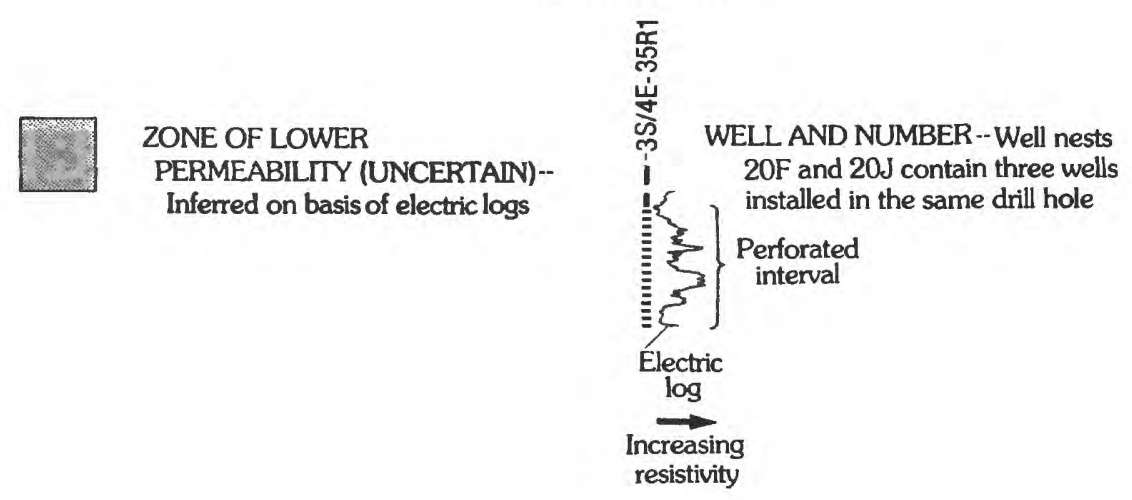

Figure 23. Generalized geohydrologic section showing perforated intervals of wells near the artificial-recharge area. 
3S/4E-34R1 and -36M1 during the modelsimulation period.

Examination of figure 23, along with the measured water-quality data, yields a possible explanation for this faster-than-expected rise in dissolved-solids concentration at some downgradient wells and for the differences in waterquality responses among wells in the same area. The suggested explanation is that the recharge water is moving much more rapidly through the uppermost part of the ground-water system than through deeper parts.

In the artificial-recharge area, all the shallow wells--those with perforations above the uppermost zone of lower permeability in figure 23-show significant increases in dissolved-solids concentration. The two multilevel well nests (3S/4E-20F and $-20 \mathrm{~J})$ provide information on the vertical differences in water quality in the artificial-recharge area. Each nest consists of three wells installed in the same drill hole. Dissolved-solids concentration of water from well 3S/4E-20F1 perforated at a shallow depth in nest $3 \mathrm{~S} / 4 \mathrm{E}-20 \mathrm{~F}$ rose in response to the artificial recharge. The other two wells (3S/4E20F2 and -20F3), perforated at greater depths, seem to show no concentration increase. The initially high dissolved-solids concentration in well 3S/4E-20F3 may be due to a different source, such as residual drilling mud, because these two wells were not fully developed until 1986. The dissolved-solids concentration of water from three wells in nest 3S/4E-20J increased during 1983-87; however, the concentration increase took longer to reach wells at greater depths, and the increase diminished in magnitude with depth.

Wells 3S/4E-34H1, -34R1, -35R1, -35R2, $-36 \mathrm{M} 1$, and $4 \mathrm{~S} / 4 \mathrm{E}-2 \mathrm{~B} 1$ are downgradient from the artificial-recharge area. Water from wells 3S/4E-34R1 and -36M1, which have the shallowest perforations, had the most rapid rise in dissolved-solids concentration. Water from wells
3S/4E-35R1, -35R2, and 4S/4E-2B1, which have slightly deeper perforations, had a more delayed water-quality response. Water from well 3S/4E-34H1, which has the deepest perforations, had a water-quality response similar to those of 3S/4E-35R1 and -35R2, although well $3 \mathrm{~S} / 4 \mathrm{E}-34 \mathrm{H} 1$ is about a mile closer to the recharge area. Any analysis of water quality at these six downgradient wells is complicated by the fact that they all are pumped wells. Waterquality differences among these wells may be due partly to differences in pumping rates (see appendix 2).

The sparse water-quality data suggest that transport occurs in the upper part of the aquifer at rates much higher than those predicted by the solute-transport submodel, which is a twodimensional areal model that assumes complete mixing through the entire depth of the aquifer. The existing model, however, can be used as a tool to qualitatively demonstrate the transport effects of a much more permeable upper zone. A second transport simulation was completed in which it was assumed that all flow occurs in the upper 20 percent of the aquifer. In model input, all thicknesses were divided by 5 and all conductivities were multiplied by 5 to maintain the same transmissivity. Storage-coefficient values were not affected (see appendix 6).

Measured dissolved-solids concentrations and those simulated when it is assumed that all transport occurs in the upper ( 20 percent) part of the aquifer are shown in figures 24 and 25. For several wells, this assumption seems to yield model results closer to measured values than those simulated under the assumption of complete mixing through the entire depth of the aquifer (see figs. 21 and 22). This is particularly true for the shallow wells in the artificialrecharge area. For downgradient wells (3S/4E$34 \mathrm{H} 1,-34 \mathrm{R} 1,-35 \mathrm{R} 1,-35 \mathrm{R} 2,-36 \mathrm{M} 1$, and $4 \mathrm{~S} / 4 \mathrm{E}-$ $2 \mathrm{~B} 1$ ), there is a divergence between simulated and measured dissolved-solids concentrations 

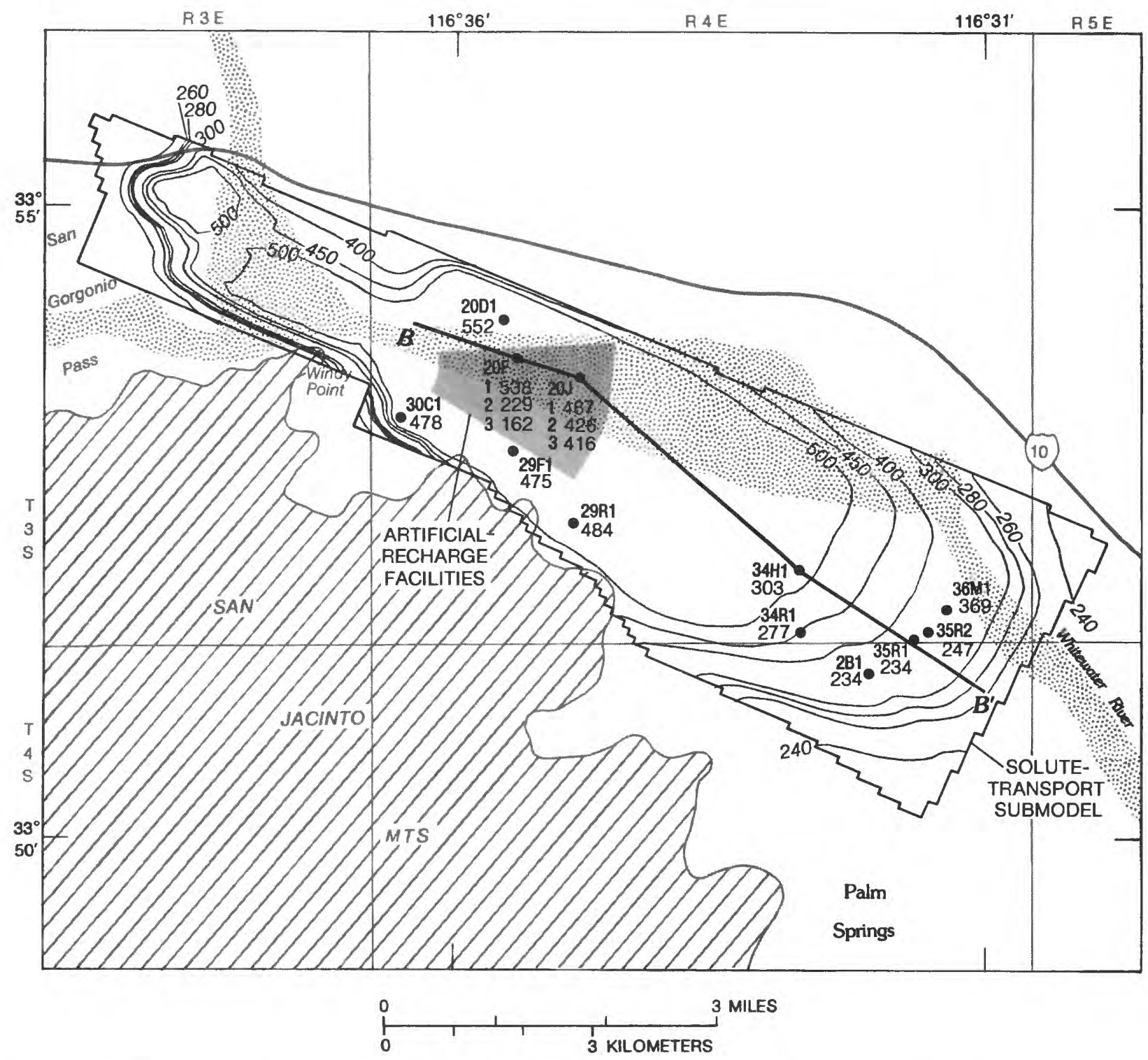

EXPLANATION

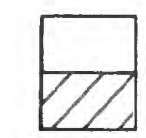

B

$\boldsymbol{B}^{\prime}$ LINE OF GEOHYDROLOGIC SECTION--

Shown in figure 23

UNCONSOLIDATED AND PARTLY CONSOLIDATED DEPOSITS CONSOLIDATED ROCKS

\author{
- 260 — LINE OF EQUAL SIMULATED DISSOLVED. \\ SOLIDS CONCENTRATION--Interval \\ variable, in milligrams per liter \\ - 2B1 WELL AND NUMBER-Well nests 20F and \\ $20 \mathrm{~J}$ contain three wells installed in the same \\ drill hole \\ MEASURED DISSOLVED-SOLIDS \\ CONCENTRATION--In milligrams per liter
}

Figure 24. Simulated dissolved-solids concentration, assuming that all transport occurs in the upper 20 percent of the aquifer, and measured dissolved-solids concentration, autumn 1986. 

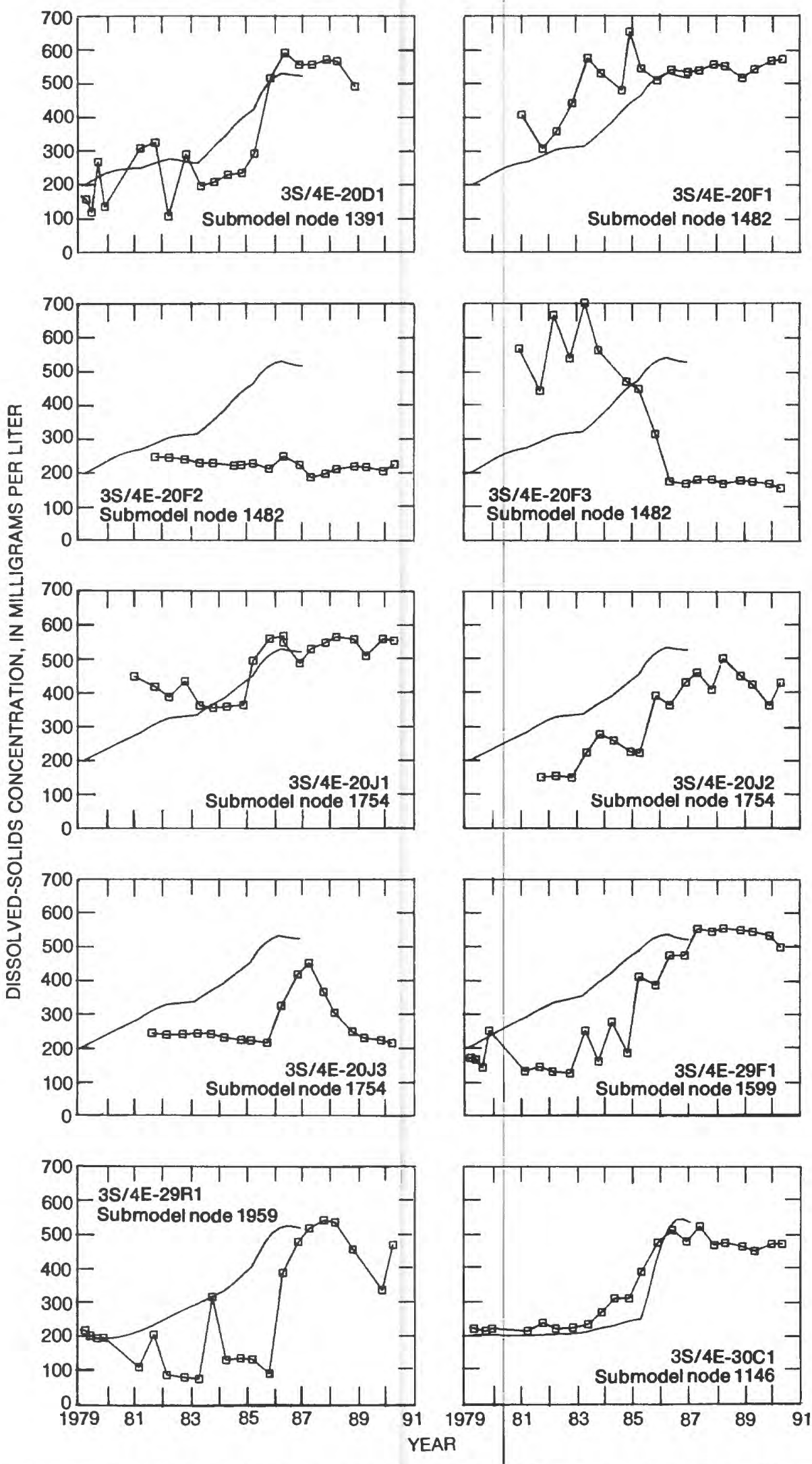

Figure 25. Simulated dissolved-solids concentration, 1979-86, assuming that all transport occurs in the upper 20 percent of the aquifer, and measured dissolved-solids concentration at selected wells. 


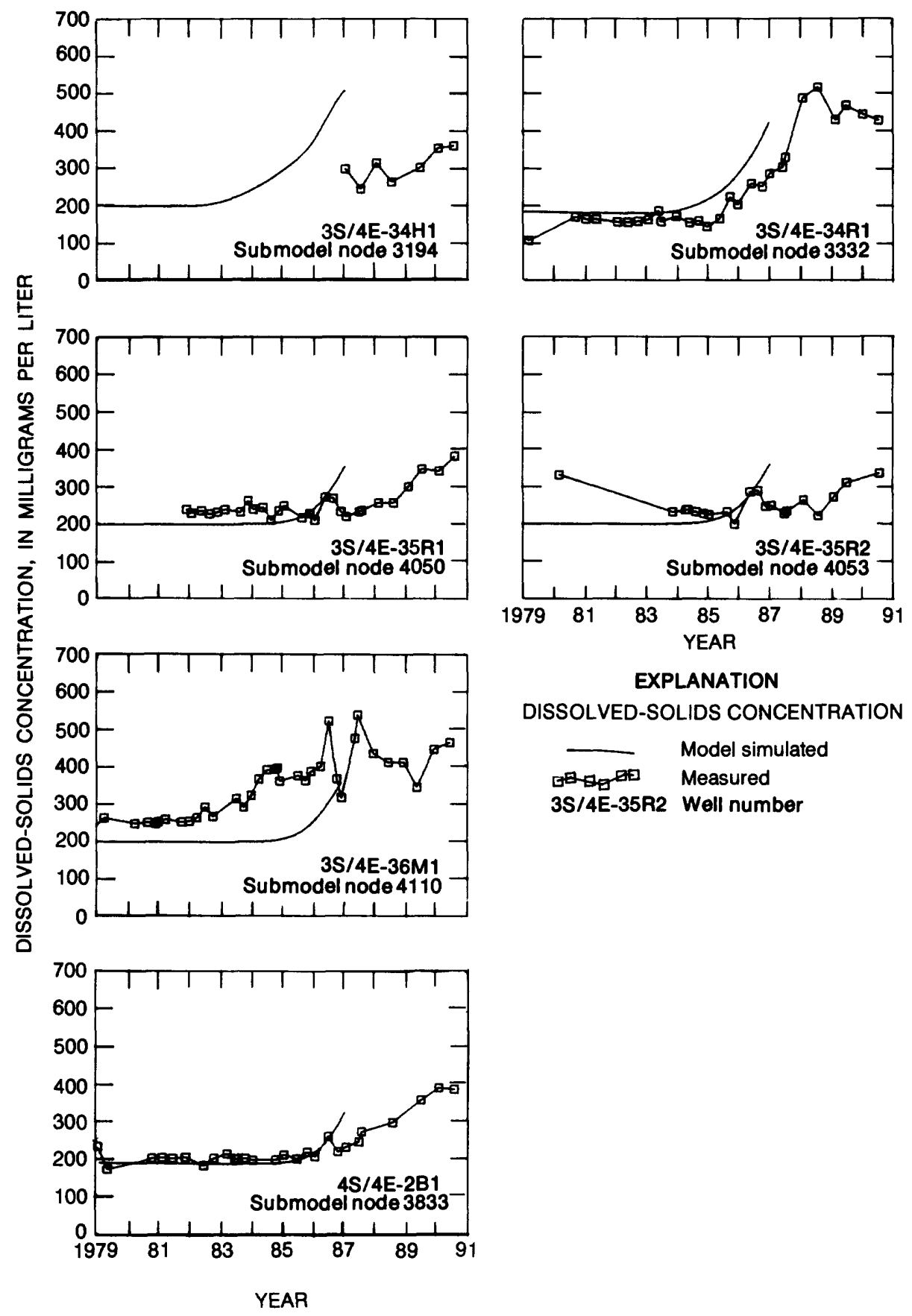

Figure 25. Continued. 
that correlates with the depth of the top of perforations. For example, the model overestimates the arrival time of the high dissolved-solids water at well 3S/4E-36M1, which is the shallowest of the downgradient wells, and underestimates the arrival time for all the deeper downgradient wells. The deeper the top of the perforations, the larger the underestimation error.

Although no quantitative conclusions can be drawn from the transport simulations, the results suggest that there are significant vertical differences in transport rates: Most of the artificial-recharge water appears to flow through the upper, more permeable part of the aquifer. To test this finding would require additional water-quality sampling and water-level measuring at multiple depths, as well as cross-sectional and (or) three-dimensional flow and transport modeling.

\section{CONCLUSIONS}

\section{Model Evaluation}

Several conclusions can be drawn from the model evaluation:

\section{General Conclusions--}

(1) Even extensively calibrated models may yield inaccurate results when used to simulate time periods in which stress conditions were significantly different from those of the calibration period.

(2) Vertical differences in solute-transport rates are likely to be very important, even when flow is adequately simulated with an areal two-dimensional model.

\section{Specific Conclusions--}

(1) The large rise in water levels in 198586 had a significant effect on the groundwater flow system. Saturated thickness in the artificial-recharge area increased by as much as $400 \mathrm{ft}$, causing an increase in transmissivity and a change in the storage coefficient.

(2) Extending the model to the true southeastern boundary of the flow system, the Salton Sea, would enable adequate simulation of the flow system. The assumed constant-head boundary at the southeastern end of the upper Coachella Valley is no longer appropriate. The specified-head boundary used for the retrospective 1979-86 simulations in this study is not appropriate for any predictive model simulations.

(3) Revised estimates for several components of the upper-valley water budget could allow more accurate analysis of the effects of future water development. In particular, the rates of recharge from the streams flowing out of the San Jacinto Mountains and underflow from the San Gorgonio Pass during 1979-86 seem to have been higher than the estimates used in previous studies.

(4) There is uncertainty about the factor to use to convert gross pumpage to net pumpage. The appropriate value to use in the Coachella Valley could be determined through additional study.

(5) The monthly water budget of the artificial-recharge ponds would need to be documented to enable determination of the actual amount of diverted water that recharges the ground-water system.

(6) Important vertical differences in transport are inadequately simulated by the areal two-dimensional solute-transport submodel. In particular, solutes may be moving much more rapidly in the uppermost part of the ground-water system relative to deeper parts. 


\section{Future Data Collection}

Additional data would add to the understanding of the three-dimensional flow system of the entire Coachella Valley. The drilling of several sets of nested wells would allow for measurement of dissolved-solids concentrations and water levels at varying depths. Such additional data would be most useful for the following areas:

- upgradient of the artificial-recharge area

- just downgradient of the artificial-recharge area

- at the base of Palm Canyon

- at the base of Deep Canyon

- at the lower end of the existing-model area (northwest of Indio)

- in the lower valley

- on both sides of major faults in the valley

Aquifer tests at wells in these areas would improve understanding of three-dimensional geohydrologic properties in the valley.

Existing water-quality data in the valley are sparse. A water-quality monitoring network could provide information on: (1) regional water-quality trends, (2) the effects of identified potential contamination sources on groundwater quality, and (3) vertical water-quality differences. The multilevel sampling of newly drilled test holes, along with regular waterquality sampling of a number of existing wells, could provide valuable information.

\section{Future Modeling}

A basinwide multilayer flow model and a set of submodels of chemical transport would be useful in evaluating the three-dimensional effects of ground-water development on water levels and water quality. A basinwide flow model would include both the upper and lower valleys. The modeling of the upper valley could be based, in part, on the model evaluated in this report, but it could be enhanced in several ways. First, it could incorporate vertical differences in the ground-water flow system. Second, it could allow simulation of unconfined conditions, where transmissivity is a function of water level. Third, the model parameters--storage coefficient, hydraulic conductivity, aquifer thickness, dispersivity, and porosity--could be reestimated in light of the results of this report and the analysis of new and preexisting data. Fourth, the assumed basin water budget could be revised on the basis of the results of this report and extended from two dimensions to three. As described above, refined estimates of stream recharge, artificial recharge, and return flow from pumpage would allow more accurate model simulations.

Extending the model to the basin's true southeastern boundary, the Salton Sea, could aid in understanding the valleywide hydrologic system. The lower valley traditionally has been described as a multiaquifer system consisting of an upper "perched" zone, a confining layer of fine-textured lake deposits, an upper aquifer, a confining unit, and a lower aquifer. Most production is from the lower aquifer. The existing data base of well logs, water-level measurements, and water quality is inadequate to determine the areal extent of hydraulically distinguishable layers. In addition, the water budget in the lower valley has not been defined adequately. Estimates of inflow from the various ungaged streams and of the large amount of irrigation water that is recharging the system are needed to accurately model the movement of ground water in the lower valley.

A second important modeling need is the development of a new transport model (or models). A new generalized regional transport model could help provide a greater understanding of regional water-quality trends. Regional water-quality issues include the effects of the artificially recharged Colorado River water in the upper valley, potential nonpoint agricultural contaminant sources in the lower valley, and "natural" geochemical changes in the ground 
water as it moves through the valley. As indicated in this report, the existing twodimensional model of the upper valley has proved helpful in analyzing the potential areal movement of the artificial-recharge water, but it cannot simulate the vertical differences in solute transport.

Several possible modeling approaches are available to address vertical differences in the movement of chemicals. One approach is to use cross-sectional models that can simulate chemical transport along ground-water flow lines. A cross-sectional model in the artificial-recharge area could be particularly helpful in testing the hypothesis that the high-dissolved-solids water is moving more rapidly in the upper part of the ground-water system.

A second, more computationally intensive approach is to use actual three-dimensional or layered models. Simulations carried out as part of this study indicate that cell size must be quite small (several hundred feet per side) to accurately model advective-dispersive transport. Areally extensive three-dimensional modeling may therefore be computationally infeasible.

A third approach to transport modeling is to focus strictly on advective transport. This could be done by linking a particle-tracking algorithm with a three-dimensional flow model (see, for example, Pollock, 1989). Given that advection is likely to be the dominant transport process and that dispersion is very difficult to quantify, such an approach seems potentially useful in the Coachella Valley.

\section{REFERENCES CITED}

Allen, C.R., 1957, San Andreas fault zone in San Gorgonio Pass, southern California: Geological Society of America Bulletin, v. 68, March, 1957, p. 315-350.

Brown, J.S., 1923, The Salton Sea region, California--a geographic, geologic, and hydrologic reconnaissance, with a guide to desert watering places: U.S. Geological Survey Water-Supply Paper 497, 292 p.

California Department of Water Resources, 1964, Coachella Valley investigation: Bulletin 108, 145 p.
California Department of Water Resources, 1979, Coachella Valley area well standards investigation: Southern District, $40 \mathrm{p}$.

--1987, California water: Looking to the future: Bulletin 160-87, 122 p.

----1988, California water: Looking to the future, statistical appendix: Bulletin 160-87, 30 p.

Dibblee, T.W., Jr., 1954, Geology of the Imperial Valley Region, in Jahns, R.H., ed., Geology of southern California: California Division of Mines Bulletin 170, chap. 2, p. 21-28.

Hely, A.G., and Peck, E.L., 1964, Precipitation, runoff, and water loss in the lower Colorado River-Salton Sea area: U.S. Geological Survey Professional Paper 486-B, 16 p.

Huberty, M.R., Pillsbury, A.F., and Sokoloff, V.P., 1948, Hydrologic studies in Coachella Valley, California: Berkeley, California, University of California, College of Agriculture, Agricultural Experiment Station, $31 \mathrm{p}$.

Konikow, L.F., and Bredehoeft, J.D., 1978, Computer model of two-dimensional solute transport and dispersion in ground water: U.S. Geological Survey Techniques of Water-Resources Investigations, Book 7 , chap. $\mathrm{C} 2,90 \mathrm{p}$.

Levy, T.E., 1988, Ground water basin management and artificial recharge: American Society of Civil Engineers, Irrigation Division, Symposium on Artificial Recharge of Ground Water, Anaheim, California, 1988, Proceedings, p. 455-464.

Loeltz, O.J., Irelan, Burdge, Robison, J.H., and Olmsted, F.H., 1975, Geohydrologic reconnaissance of the Imperial Valley, California: U.S. Geological Survey Professional Paper 486-K, 54 p.

Mendenhall, W.C., 1909, Ground waters of the Indio region, California, with a sketch of the California desert: U.S. Geological Survey Water-Supply Paper 225, $56 \mathrm{p}$.

Pollock, D.W., 1989, Documentation of computer programs to compute and display pathlines using results from the U.S. Geological Survey modular threedimensional finite difference ground-water flow model: U.S. Geological Survey Open-File Report 89-381, $188 \mathrm{p}$.

Proctor, R.J., 1968, Geology of the Desert Hot Springsupper Coachella Valley area, California: California Division of Mines, Special Report 94, 50 p.

Swain, L.A., 1978, Predicted water-level and water-quality effects of artificial recharge in the upper Coachella Valley, California, using a finite-element digital model: U.S. Geological Survey Water-Resources Investigations Report 77-29, 54 p.

Tyley, S.J., 1974, Analog model study of the ground-water basin of the upper Coachella Valley, California: U.S. Geological Survey Water-Supply Paper 2027, 77 p.

Voss, C.I., 1984, A finite-element simulation model for saltwater-unsaturated, fluid-density-dependent groundwater flow with energy transport on chemically-reactive single-species solute transport: U.S. Geological Survey Water-Resources Investigations Report 84-4369, 409 p. 
Appendix 1: List and description of wells in data base

[Remarks: L, water-level well; P, well with $1979-86$ pumpage data; Q, water-quality well; U, sampled by U.S. Geological Survey; --, no data]

$\begin{array}{ccccc}\text { Well } & \begin{array}{c}\text { Perforated } \\
\text { interval (depth, in } \\
\text { feet below land } \\
\text { surface) }\end{array} & \begin{array}{c}\text { Land-surface } \\
\text { altitude (feet } \\
\text { above or } \\
\text { below (-) sea } \\
\text { level) }\end{array} & \text { Latitude } & \text { Longitude }\end{array} \quad$\begin{tabular}{c}
$\begin{array}{c}\text { Closest } \\
\text { model node } \\
\text { for pumped } \\
\text { wells (in } \\
\text { model area } \\
\text { only) }\end{array}$ \\
\hline
\end{tabular}

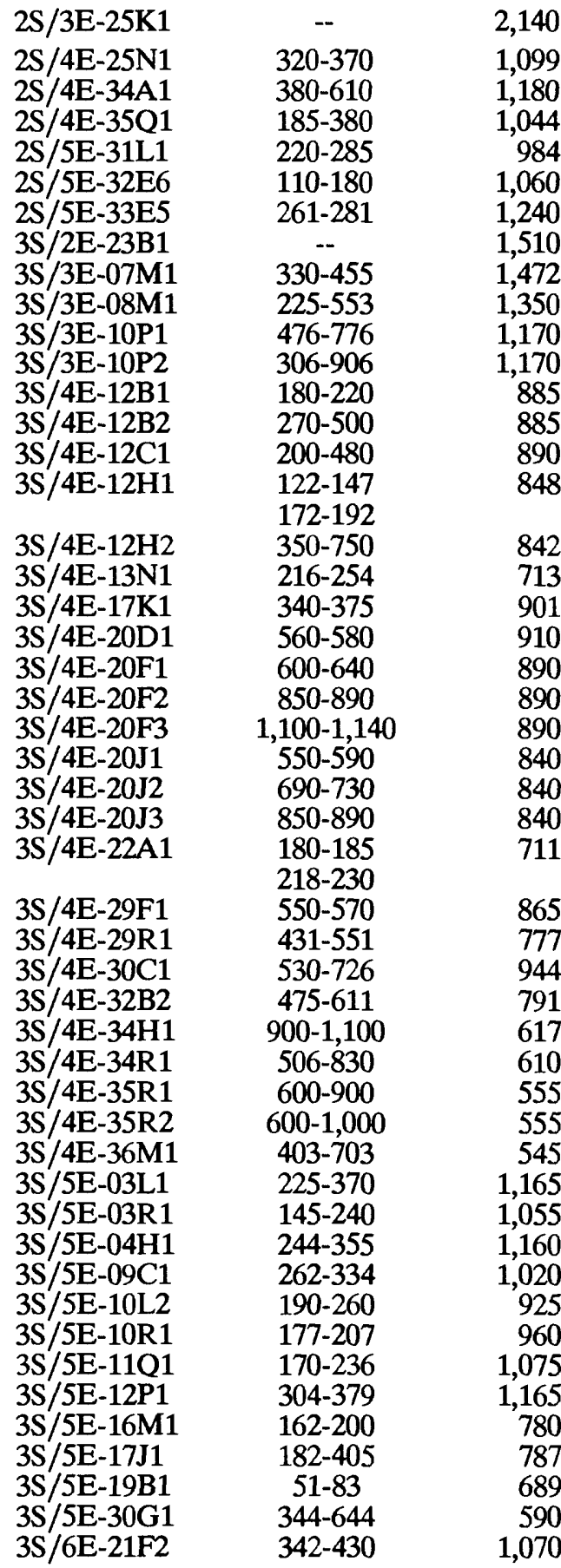

$33^{\circ} 58^{\prime} 04^{\prime \prime} \mathrm{N} \quad 116^{\circ} 37^{\prime} 10^{\prime \prime} \mathrm{W}$

$33^{\circ} 57^{\prime} 42^{\prime \prime} \mathrm{N} \quad 116^{\circ} 31^{\prime} 22^{\prime \prime} \mathrm{W}$

$33^{\circ} 57^{\prime} 34^{\prime \prime} \mathrm{N} \quad 116^{\circ} 32^{\prime} 49^{\prime \prime} \mathrm{W}$

$33^{\circ} 56^{\prime} 59^{\prime \prime} \mathrm{N} \quad 116^{\circ} 31^{\prime} 53^{\prime \prime} \mathrm{W}$

$33^{\circ} 57^{\prime} 08^{\prime \prime} \mathrm{N} \quad 116^{\circ} 30^{\prime} 09^{\prime \prime} \mathrm{W}$

$33^{\circ} 57^{\prime} 15^{\prime \prime} \mathrm{N} \quad 116^{\circ} 29^{\prime} 15^{\prime \prime} \mathrm{W}$

$33^{\circ} 57^{\prime} 17^{\prime \prime} \mathrm{N} \quad 116^{\circ} 28^{\prime} 21^{\prime \prime} \mathrm{W}$

$33^{\circ} 54^{\prime} 11^{\prime \prime} \mathrm{N} 116^{\circ} 44^{\prime} 34^{\prime \prime} \mathrm{W}$

$33^{\circ} 55^{\prime} 22^{\prime \prime} \mathrm{N} \quad 116^{\circ} 43^{\prime} 06^{\prime \prime} \mathrm{W}$

$33^{\circ} 55^{\prime} 19^{\prime \prime} \mathrm{N} \quad 116^{\circ} 41^{\prime} 54^{\prime \prime} \mathrm{W}$

$33^{\circ} 55^{\prime} 06^{\prime \prime} \mathrm{N} \quad 116^{\circ} 39^{\prime} 41^{\prime \prime} \mathrm{W}$

$33^{\circ} 55^{\prime} 06^{\prime \prime} \mathrm{N} \quad 116^{\circ} 39^{\prime} 42^{\prime \prime} \mathrm{W}$

$33^{\circ} 55^{\prime} 53^{\prime \prime} \mathrm{N} \quad 116^{\circ} 30^{\prime} 51^{\prime \prime} \mathrm{W}$

$33^{\circ} 55^{\prime} 55^{\prime \prime} \mathrm{N} \quad 116^{\circ} 30^{\prime} 51^{\prime \prime} \mathrm{W}$

$33^{\circ} 55^{\prime} 54^{\prime \prime} \mathrm{N} \quad 116^{\circ} 31^{\prime} 06^{\prime \prime} \mathrm{W}$

$33^{\circ} 55^{\prime} 34^{\prime \prime} \mathrm{N} 116^{\circ} 30^{\prime} 48^{\prime \prime} \mathrm{W}$

$33^{\circ} 55^{\prime} 36^{\prime \prime} \mathrm{N} \quad 116^{\circ} 30^{\prime} 42^{\prime \prime} \mathrm{W}$

$33^{\circ} 54^{\prime} 13^{\prime \prime} \mathrm{N} \quad 116^{\circ} 31^{\prime} 28^{\prime \prime} \mathrm{W}$

$33^{\circ} 54^{\prime} 36^{\prime \prime} \mathrm{N} \quad 116^{\circ} 35^{\prime} 13^{\prime \prime} \mathrm{W}$

$33^{\circ} 54^{\prime} 07^{\prime \prime} \mathrm{N} \quad 116^{\circ} 35^{\prime} 34^{\prime \prime} \mathrm{W}$

$33^{\circ} 53^{\prime} 47^{\prime \prime} \mathrm{N} \quad 116^{\circ} 35^{\prime} 27^{\prime \prime} \mathrm{W}$

$33^{\circ} 53^{\prime} 47^{\prime \prime} \mathrm{N} \quad 116^{\circ} 35^{\prime} 27^{\prime \prime} \mathrm{W}$

$33^{\circ} 53^{\prime} 47^{\prime \prime} \mathrm{N} \quad 116^{\circ} 35^{\prime} 27^{\prime \prime} \mathrm{W}$

$33^{\circ} 53^{\prime} 39^{\prime \prime} \mathrm{N} \quad 116^{\circ} 34^{\prime} 53^{\prime \prime} \mathrm{W}$

$33^{\circ} 53^{\prime} 39^{\prime \prime} \mathrm{N} 116^{\circ} 34^{\prime} 53^{\prime \prime} \mathrm{W}$

$33^{\circ} 53^{\prime} 39^{\prime \prime} \mathrm{N} \quad 116^{\circ} 34^{\prime} 53^{\prime \prime} \mathrm{W}$

$33^{\circ} 54^{\prime} 09^{\prime \prime} \mathrm{N} 116^{\circ} 32^{\prime} 43^{\prime \prime} \mathrm{W}$

$33^{\circ} 53^{\prime} 05^{\prime \prime} \mathrm{N} \quad 116^{\circ} 35^{\prime} 28^{\prime \prime} \mathrm{W}$

$33^{\circ} 52^{\prime} 30^{\prime \prime} \mathrm{N} 116^{\circ} 34^{\prime} 53^{\prime \prime} \mathrm{W}$

$33^{\circ} 53^{\prime} 20^{\prime \prime} \mathrm{N} \quad 116^{\circ} 36^{\prime} 32^{\prime \prime} \mathrm{W}$

$33^{\circ} 52^{\prime} 20^{\prime \prime} \mathrm{N} \quad 116^{\circ} 35^{\prime} 09^{\prime \prime} \mathrm{W}$

$33^{\circ} 52^{\prime} 09^{\prime \prime} \mathrm{N} 116^{\circ} 32^{\prime} 46^{\prime \prime} \mathrm{W}$

$33^{\circ} 51^{\prime} 39^{\prime \prime} \mathrm{N} \quad 116^{\circ} 32^{\prime} 46^{\prime \prime} \mathrm{W}$

$33^{\circ} 51^{\prime} 34^{\prime \prime} \mathrm{N} \quad 116^{\circ} 31^{\prime} 39^{\prime \prime} \mathrm{W}$

$33^{\circ} 51^{\prime} 41^{\prime \prime} \mathrm{N} \quad 116^{\circ} 31^{\prime} 39^{\prime \prime} \mathrm{W}$

$33^{\circ} 51^{\prime} 52^{\prime \prime} \mathrm{N} \quad 116^{\circ} 31^{\prime} 29^{\prime \prime} \mathrm{W}$

$33^{\circ} 56^{\prime} 21^{\prime \prime} \mathrm{N} \quad 116^{\circ} 27^{\prime} 08^{\prime \prime} \mathrm{W}$

$33^{\circ} 55^{\prime} 57^{\prime \prime} \mathrm{N} \quad 116^{\circ} 26^{\prime} 29^{\prime \prime} \mathrm{W}$

$33^{\circ} 56^{\prime} 25^{\prime \prime} \mathrm{N} \quad 116^{\circ} 27^{\prime} 26^{\prime \prime} \mathrm{W}$

$33^{\circ} 55^{\prime} 55^{\prime \prime} \mathrm{N} \quad 116^{\circ} 27^{\prime} 58^{\prime \prime} \mathrm{W}$

$33^{\circ} 55^{\prime} 18^{\prime \prime} \mathrm{N} \quad 116^{\circ} 27^{\prime} 03^{\prime \prime} \mathrm{W}$

$33^{\circ} 55^{\prime} 15^{\prime \prime} \mathrm{N} \quad 116^{\circ} 26^{\prime} 31^{\prime \prime} \mathrm{W}$

$33^{\circ} 55^{\prime} 15^{\prime \prime} \mathrm{N} \quad 116^{\circ} 25^{\prime} 42^{\prime \prime} \mathrm{W}$

$33^{\circ} 55^{\prime} 10^{\prime \prime} \mathrm{N} \quad 116^{\circ} 25^{\prime} 03^{\prime \prime} \mathrm{W}$

$33^{\circ} 54^{\prime} 27^{\prime \prime} \mathrm{N} \quad 116^{\circ} 28^{\prime} 24^{\prime \prime} \mathrm{W}$

$33^{\circ} 54^{\prime} 36^{\prime \prime} \mathrm{N} \quad 116^{\circ} 28^{\prime} 30^{\prime \prime} \mathrm{W}$

$33^{\circ} 54^{\prime} 08^{\prime \prime} \mathrm{N} \quad 116^{\circ} 29^{\prime} 48^{\prime \prime} \mathrm{W}$

$33^{\circ} 53^{\prime} 00^{\prime \prime} \mathrm{N} 116^{\circ} 29^{\prime} 53^{\prime \prime} \mathrm{W}$

$33^{\circ} 53^{\prime} 54^{\prime \prime} \mathrm{N} \quad 116^{\circ} 21^{\prime} 49^{\prime \prime} \mathrm{W}$
L

L

L

$\mathrm{L}$

L

$\mathrm{L}$

L

L

L

LPQ $\quad 25$

LP

LP

LP

L

L

L

$\mathrm{L}$

LQU

LQU

LQU

LQU

LQU

LQU

LQU

LQU

LQU

LPQU $\quad 76$

$\mathrm{L}$

$\begin{array}{ll}\text { LPQ } & 109 \\ \text { LPQ } & 119\end{array}$

LPQ 120

LPQ 120

LPQ 120

L

L

L

L

L

L

L

L

L.

L

L 
Appendix 1: List and description of wells in data base--Continued

\begin{tabular}{|c|c|c|c|c|c|c|}
\hline $\begin{array}{l}\text { Well } \\
\text { number }\end{array}$ & $\begin{array}{c}\text { Perforated } \\
\text { interval (depth, in } \\
\text { feet below land } \\
\text { surface) }\end{array}$ & $\begin{array}{l}\text { Land-surface } \\
\text { altitude (feet } \\
\text { above or } \\
\text { below (-) sea } \\
\text { level) }\end{array}$ & Latitude & Longitude & Remarks & $\begin{array}{l}\text { Closest } \\
\text { model node } \\
\text { for pumped } \\
\text { wells (in } \\
\text { model area } \\
\text { only) }\end{array}$ \\
\hline 3S/6E-25Q1 & $371-511$ & 955 & $33^{\circ} 52^{\prime} 31^{\prime \prime} \mathrm{N}$ & $116^{\circ} 18^{\prime} 27^{\prime \prime} \mathrm{W}$ & $\mathrm{L}$ & \\
\hline $3 S / 6 E-26 P 1$ & $204-310$ & 960 & $33^{\circ} 52^{\prime} 35^{\prime \prime} \mathrm{N}$ & $116^{\circ} 19^{\prime} 46^{\prime \prime} \mathrm{W}$ & $\mathrm{L}$ & \\
\hline $3 S / 6 E-28 A 1$ & $250-332$ & 1,000 & $33^{\circ} 53^{\prime} 10^{\prime \prime} \mathrm{N}$ & $116^{\circ} 21^{\prime} 40^{\prime \prime} \mathrm{W}$ & $\mathrm{L}$ & \\
\hline 3S'/6E-36P1 & -- & 772 & $33^{\circ} 51^{\prime} 38^{\prime \prime} \mathrm{N}$ & $116^{\circ} 18^{\prime} 43^{\prime \prime} \mathrm{W}$ & $\mathrm{L}$ & \\
\hline $4 \mathrm{~S} / 4 \mathrm{E}-01 \mathrm{~B} 1$ & -- & 510 & $33^{\circ} 51^{\prime} 27^{\prime \prime} \mathrm{N}$ & $116^{\circ} 30^{\prime} 59^{\prime \prime} \mathrm{W}$ & $\mathbf{P}$ & 131 \\
\hline 4S/4E-01B2 & -- & 510 & $33^{\circ} 51^{\prime} 26^{\prime \prime} \mathrm{N}$ & $116^{\circ} 30^{\prime} 59^{\prime \prime} \mathrm{W}$ & $\mathbf{P}$ & 131 \\
\hline $4 S / 4 E-01 B 3$ & $400-700$ & 510 & $33^{\circ} 51^{\prime} 28^{\prime \prime} \mathrm{N}$ & $116^{\circ} 31^{\prime} 01^{\prime \prime} \mathrm{W}$ & $\mathrm{L}$ & \\
\hline $4 \mathrm{~S} / 4 \mathrm{E}-01 \mathrm{~N} 2$ & $525-800$ & 500 & $33^{\circ} 50^{\prime} 42^{\prime \prime} \mathrm{N}$ & $116^{\circ} 31^{\prime} 26^{\prime \prime} \mathrm{W}$ & LPQ & 130 \\
\hline 4S/4E-02B1 & $570-1,003$ & 565 & $33^{\circ} 51^{\prime} 22^{\prime \prime} \mathrm{N}$ & $116^{\circ} 32^{\prime} 07^{\prime \prime} \mathrm{W}$ & LPQ & 119 \\
\hline $4 \mathrm{~S} / 4 \mathrm{E}-11 \mathrm{~K} 1$ & -- & 493 & $33^{\circ} 50^{\prime} 09^{\prime \prime} \mathrm{N}$ & $116^{\circ} 32^{\prime} 08^{\prime \prime} \mathrm{W}$ & LPQ & 128 \\
\hline $4 S^{\prime} / 4 \mathrm{E}-11 \mathrm{Q} 1$ & $302-402$ & 470 & $33^{\circ} 49^{\prime} 49^{\prime \prime} \mathrm{N}$ & $116^{\circ} 32^{\prime} 08^{\prime \prime} \mathrm{W}$ & LPQ & 128 \\
\hline $4 S / 4 E-11 Q 2$ & $535-948$ & 468 & $33^{\circ} 49^{\prime} 51^{\prime \prime} \mathrm{N}$ & $116^{\circ} 32^{\prime} 08^{\prime \prime} \mathrm{W}$ & LPQ & 128 \\
\hline $4 \mathrm{~S} / 4 \mathrm{E}-11 \mathrm{R} 1$ & $526-700$ & 458 & $33^{\circ} 49^{\prime} 50^{\prime \prime} \mathrm{N}$ & $116^{\circ} 31^{\prime} 38^{\prime \prime} \mathrm{W}$ & LPQ & 128 \\
\hline $4 S / 4 E-13 C 1$ & $512-912$ & 450 & $33^{\circ} 49^{\prime} 48^{\prime \prime} \mathrm{N}$ & $116^{\circ} 31^{\prime} 21^{\prime \prime} \mathrm{W}$ & LPQ & 129 \\
\hline $4 S^{\prime} / 4 \mathrm{E}-13 \mathrm{H} 1$ & $400-500$ & 418 & $33^{\circ} 49^{\prime} 28^{\prime \prime} \mathrm{N}$ & $116^{\circ} 30^{\prime} 42^{\prime \prime} \mathrm{W}$ & $\mathrm{L}$ & \\
\hline $4 \mathrm{~S} / 4 \mathrm{E}-13 \mathrm{P} 1$ & $\begin{array}{l}345-365 \\
398-408\end{array}$ & 410 & $33^{\circ} 49^{\prime} 06^{\prime \prime} \mathrm{N}$ & $116^{\circ} 31^{\prime} 22^{\prime \prime} \mathrm{W}$ & L & \\
\hline 4S/4E-13Q1 & -- & 400 & $33^{\circ} 49^{\prime} 03^{\prime \prime} \mathrm{N}$ & $116^{\circ} 30^{\prime} 59^{\prime \prime} \mathrm{W}$ & $\mathrm{PQ}$ & 142 \\
\hline 4S/4E-14Q1 & $\begin{array}{l}622-830 \\
850-958\end{array}$ & 425 & $33^{\circ} 49^{\prime} 05^{\prime \prime} \mathrm{N}$ & $116^{\circ} 32^{\prime} 07^{\prime \prime} \mathrm{W}$ & LPQ & 141 \\
\hline 4S/4E-14R1 & $560-800$ & 408 & $33^{\circ} 49^{\prime} 03^{\prime \prime} \mathrm{N}$ & $116^{\circ} 31^{\prime} 42^{\prime \prime} \mathrm{W}$ & LPQ & 142 \\
\hline $4 \mathrm{~S} / 4 \mathrm{E}-15 \mathrm{~J} 1$ & $\begin{array}{l}164-344 \\
415-435\end{array}$ & 453 & $33^{\circ} 49^{\prime} 20^{\prime \prime} \mathrm{N}$ & $116^{\circ} 32^{\prime} 48^{\prime \prime} \mathrm{W}$ & $\mathrm{L}$ & \\
\hline 4S/4E-23D1 & $190-408$ & 435 & $33^{\circ} 48^{\prime} 45^{\prime \prime} \mathrm{N}$ & $116^{\circ} 32^{\prime} 24^{\prime \prime} \mathrm{W}$ & LPQ & 141 \\
\hline 4S/4E-23E1 & $240-472$ & 435 & $33^{\circ} 48^{\prime} 42^{\prime \prime} \mathrm{N}$ & $116^{\circ} 32^{\prime} 28^{\prime \prime} \mathrm{W}$ & LPQ & 141 \\
\hline 4S/4E-24D1 & $580-980$ & 398 & $33^{\circ} 48^{\prime} 46^{\prime \prime} \mathrm{N}$ & $116^{\circ} 31^{\prime} 32^{\prime \prime} \mathrm{W}$ & LPQ & 141 \\
\hline $4 \mathrm{~S} / 4 \mathrm{E}-24 \mathrm{H} 1$ & $600-1,000$ & 390 & $33^{\circ} 48^{\prime} 34^{\prime \prime} \mathrm{N}$ & $116^{\circ} 30^{\prime} 38^{\prime \prime} \mathrm{W}$ & LPQ & 142 \\
\hline $4 \mathrm{~S} / 4 \mathrm{E}-26 \mathrm{~A} 1$ & $450-780$ & 428 & $33^{\circ} 47^{\prime} 54^{\prime \prime} \mathrm{N}$ & $116^{\circ} 31^{\prime} 39^{\prime \prime} \mathrm{W}$ & LPQ & 154 \\
\hline $4 S / 4 E-26 G 1$ & $450-800$ & 440 & $33^{\circ} 47^{\prime} 48^{\prime \prime} \mathrm{N}$ & $116^{\circ} 31^{\prime} 54^{\prime \prime} \mathrm{W}$ & LPQ & 154 \\
\hline 4S/4E-35A1 & -. & 510 & $33^{\circ} 47^{\prime} 06^{\prime \prime} \mathrm{N}$ & $116^{\circ} 31^{\prime} 46^{\prime \prime} \mathrm{W}$ & $\mathrm{P}$ & 153 \\
\hline $4 \mathrm{~S} / 4 \mathrm{E}-35 \mathrm{~B} 1$ & -- & 510 & $33^{\circ} 47^{\prime} 06^{\prime \prime} \mathrm{N}$ & $116^{\circ} 32^{\prime} 02^{\prime \prime} \mathrm{W}$ & $\mathbf{P}$ & 153 \\
\hline $4 \mathrm{~S} / 4 \mathrm{E}-35 \mathrm{~K} 1$ & $435-600$ & 554 & $33^{\circ} 46^{\prime} 42^{\prime \prime} \mathrm{N}$ & $116^{\circ} 32^{\prime} 08^{\prime \prime} \mathrm{W}$ & $\mathrm{L}$ & \\
\hline 4S/4E-35L1 & -- & 540 & $33^{\circ} 46^{\prime} 40^{\prime \prime} \mathrm{N}$ & $116^{\circ} 32^{\prime} 18^{\prime \prime} \mathrm{W}$ & $\mathbf{P}$ & 138 \\
\hline 4S/5E-03P1 & $288-600$ & 380 & $33^{\circ} 50^{\prime} 51^{\prime \prime} \mathrm{N}$ & $116^{\circ} 27^{\prime} 11^{\prime \prime} \mathrm{W}$ & LP & 161 \\
\hline $4 \mathrm{~S} / 5 \mathrm{E}-04 \mathrm{E} 2$ & -- & 435 & $33^{\circ} 51^{\prime} 11^{\prime \prime} \mathrm{N}$ & $116^{\circ} 28^{\prime} 25^{\prime \prime} \mathrm{W}$ & $\mathrm{L}$ & 146 \\
\hline $4 \mathrm{~S} / 5 \mathrm{E}-04 \mathrm{~F} 1$ & $276-576$ & 430 & $33^{\circ} 51^{\prime} 17^{\prime \prime} \mathrm{N}$ & $116^{\circ} 28^{\prime} 14^{\prime \prime} \mathrm{W}$ & LP & 147 \\
\hline $4 \mathrm{~S} / 5 \mathrm{E}-04 \mathrm{~N} 1$ & $\begin{array}{l}520-690 \\
790-810 \\
860-890\end{array}$ & 425 & $33^{\circ} 50^{\prime} 54^{\prime \prime} \mathrm{N}$ & $116^{\circ} 28^{\prime} 28^{\prime \prime} \mathrm{W}$ & LP & 146 \\
\hline $4 \mathrm{~S} / 5 \mathrm{E}-05 \mathrm{~K} 1$ & $408-748$ & 446 & $33^{\circ} 51^{\prime} 00^{\prime \prime} \mathrm{N}$ & $116^{\circ} 29^{\prime} 02^{\prime \prime} \mathrm{W}$ & LP & 145 \\
\hline $4 \mathrm{~S} / 5 \mathrm{E}-08 \mathrm{~A} 1$ & $368-1,100$ & 420 & $33^{\circ} 50^{\prime} 41^{\prime \prime} \mathrm{N}$ & $116^{\circ} 28^{\prime} 32^{\prime \prime} \mathrm{W}$ & LP & 145 \\
\hline $4 \mathrm{~S} / 5 \mathrm{E}-08 \mathrm{D} 1$ & $500-900$ & 441 & $33^{\circ} 50^{\prime} 39^{\prime \prime} \mathrm{N}$ & $116^{\circ} 29^{\prime} 30^{\prime \prime} \mathrm{W}$ & $\mathrm{L}$ & 145 \\
\hline $4 \mathrm{~S} / 5 \mathrm{E}-08 \mathrm{~L} 1$ & -. & 415 & $33^{\circ} 50^{\prime} 11^{\prime \prime} \mathrm{N}$ & $116^{\circ} 29^{\prime} 05^{\prime \prime} \mathrm{W}$ & LP & 145 \\
\hline $4 \mathrm{~S} / 5 \mathrm{E}-09 \mathrm{~B} 1$ & $398-806$ & 405 & $33^{\circ} 50^{\prime} 40^{\prime \prime} \mathrm{N}$ & $116^{\circ} 27^{\prime} 58^{\prime \prime} \mathrm{W}$ & LP & 146 \\
\hline $4 \mathrm{~S} / 5 \mathrm{E}-09 \mathrm{~F} 1$ & $227-616$ & 397 & $33^{\circ} 50^{\prime} 21^{\prime \prime} \mathrm{N}$ & $116^{\circ} 28^{\prime} 02^{\prime \prime} \mathrm{W}$ & $\mathrm{L}$ & \\
\hline $4 \mathrm{~S} / 5 \mathrm{E}-09 \mathrm{F3}$ & $410-670$ & 397 & $33^{\circ} 50^{\prime} 08^{\prime \prime} \mathrm{N}$ & $116^{\circ} 28^{\prime} 06^{\prime \prime} \mathrm{W}$ & LP & 159 \\
\hline $4 \mathrm{~S} / 5 \mathrm{E}-11 \mathrm{E} 1$ & $172-607$ & 327 & $33^{\circ} 50^{\prime} 20^{\prime \prime} \mathrm{N}$ & $116^{\circ} 26^{\prime} 18^{\prime \prime} \mathrm{W}$ & LP & 160 \\
\hline $4 \mathrm{~S} / 5 \mathrm{E}-14 \mathrm{~N} 1$ & $\begin{array}{l}330-640 \\
715-780\end{array}$ & 340 & $33^{\circ} 49^{\prime} 06^{\prime \prime} \mathrm{N}$ & $116^{\circ} 26^{\prime} 16^{\prime \prime} \mathrm{W}$ & LP & 169 \\
\hline $4 \mathrm{~S} / 5 \mathrm{E}-15 \mathrm{R} 1$ & $243-507$ & 345 & $33^{\circ} 49^{\prime} 03^{\prime \prime} \mathrm{N}$ & $116^{\circ} 26^{\prime} 25^{\prime \prime} \mathrm{W}$ & LP & 169 \\
\hline $4 \mathrm{~S} / 5 \mathrm{E}-15 \mathrm{R} 2$ & $400-560$ & 346 & $33^{\circ} 49^{\prime} 08^{\prime \prime} \mathrm{N}$ & $116^{\circ} 26^{\prime} 25^{\prime \prime} \mathrm{W}$ & LP & 169 \\
\hline
\end{tabular}


Appendix 1: List and description of wells in data base--Continued

\begin{tabular}{|c|c|c|c|c|c|c|}
\hline $\begin{array}{l}\text { Well } \\
\text { number }\end{array}$ & $\begin{array}{l}\text { Perforated } \\
\text { interval (depth, in } \\
\text { feet below land } \\
\text { surface) }\end{array}$ & $\begin{array}{l}\text { Land-surface } \\
\text { altitude (feet } \\
\text { above or } \\
\text { below (-) sea } \\
\text { level) }\end{array}$ & Latitude & Longitude & Remarks & $\begin{array}{c}\text { Closest } \\
\text { model node } \\
\text { for pumped } \\
\text { wells (in } \\
\text { model area } \\
\text { only) }\end{array}$ \\
\hline 4S/5E-17Q1 & $\begin{array}{c}600-720 \\
745-795 \\
815-895 \\
920-1,000\end{array}$ & 360 & $33^{\circ} 48^{\prime} 58^{\prime \prime} \mathrm{N}$ & $116^{\circ} 28^{\prime} 47^{\prime \prime} \mathrm{W}$ & & \\
\hline 4S/5E-19D1 & $340-440$ & 393 & $33^{\circ} 51^{\prime} 52^{\prime \prime} \mathrm{N}$ & $116^{\circ} 31^{\prime} 32^{\prime \prime} \mathrm{W}$ & LPQ & 142 \\
\hline $4 \mathrm{~S} / 5 \mathrm{E}-21 \mathrm{~A} 1$ & -- & 357 & $33^{\circ} 48^{\prime} 46^{\prime \prime} \mathrm{N}$ & $116^{\circ} 27^{\prime} 35^{\prime \prime} \mathrm{W}$ & $\mathrm{L}$ & \\
\hline $4 S^{\prime} / 5 E-21 J 2$ & $\begin{array}{c}440-470 \\
680-1,000\end{array}$ & 348 & $33^{\circ} 48^{\prime} 31^{\prime \prime} \mathrm{N}$ & $116^{\circ} 27^{\prime} 34^{\prime \prime} \mathrm{W}$ & LP & 168 \\
\hline 4S/5E-22A1 & -- & 347 & $33^{\circ} 48^{\prime} 56^{\prime \prime} \mathrm{N}$ & $116^{\circ} 26^{\prime} 31^{\prime \prime} \mathrm{W}$ & $\mathrm{L}$ & \\
\hline $4 \mathrm{~S} / 5 \mathrm{E}-22 \mathrm{~B} 1$ & -- & 352 & $33^{\circ} 48^{\prime} 54^{\prime \prime} \mathrm{N}$ & $116^{\circ} 26^{\prime} 52^{\prime \prime} \mathrm{W}$ & LP & 168 \\
\hline $4 \mathrm{~S} / 5 \mathrm{E}-23 \mathrm{R} 1$ & -- & 330 & $33^{\circ} 48^{\prime} 11^{\prime \prime} \mathrm{N}$ & $116^{\circ} 25^{\prime} 29^{\prime \prime} \mathrm{W}$ & & \\
\hline $4 \mathrm{~S} / 5 \mathrm{E}-24 \mathrm{E} 1$ & $600-1,000$ & 400 & $33^{\circ} 48^{\prime} 31^{\prime \prime} \mathrm{N}$ & $116^{\circ} 31^{\prime} 37^{\prime \prime} \mathrm{W}$ & & \\
\hline $4 S^{\prime} / 5 \mathrm{E}-25 \mathrm{D} 1$ & $540-850$ & 325 & $33^{\circ} 48^{\prime} 03^{\prime \prime} \mathrm{N}$ & $116^{\circ} 25^{\prime} 08^{\prime \prime} \mathrm{W}$ & LP & 178 \\
\hline 4S/5E-25F1 & -- & 325 & $33^{\circ} 47^{\prime} 46^{\prime \prime} \mathrm{N}$ & $116^{\circ} 24^{\prime} 58^{\prime \prime} \mathrm{W}$ & LP & 178 \\
\hline $4 \mathrm{~S} / 5 \mathrm{E}-25 \mathrm{P} 1$ & $\begin{array}{l}500-740 \\
790-840\end{array}$ & 320 & $33^{\circ} 47^{\prime} 20^{\prime \prime} \mathrm{N}$ & $116^{\circ} 24^{\prime} 58^{\prime \prime} \mathrm{W}$ & $\mathrm{L}$ & 178 \\
\hline $4 \mathrm{~S} / 5 \mathrm{E}-26 \mathrm{~A} 1$ & $1,050-1,400$ & 330 & $33^{\circ} 48^{\prime} 05^{\prime \prime} \mathrm{N}$ & $116^{\circ} 25^{\prime} 22^{\prime \prime} \mathrm{W}$ & LP & 178 \\
\hline $4 \mathrm{~S} / 5 \mathrm{E}-26 \mathrm{~B} 1$ & $1,050-1,360$ & 340 & $33^{\circ} 48^{\prime} 04^{\prime \prime} \mathrm{N}$ & $116^{\circ} 25^{\prime} 48^{\prime \prime} \mathrm{W}$ & LP & 178 \\
\hline $4 S / 5 E-26 C 1$ & $\begin{array}{l}430-500 \\
660-880\end{array}$ & 342 & $33^{\circ} 47^{\prime} 56^{\prime \prime} \mathrm{N}$ & $116^{\circ} 25^{\prime} 59^{\prime \prime} \mathrm{W}$ & LP & 178 \\
\hline 4S/5E-26D1 & $769-1,385$ & 345 & $33^{\circ} 48^{\prime} 04^{\prime \prime} \mathrm{N}$ & $116^{\circ} 26^{\prime} 21^{\prime \prime} \mathrm{W}$ & LP & 168 \\
\hline $4 \mathrm{~S} / 5 \mathrm{E}-26 \mathrm{H} 1$ & $816-1,360$ & 340 & $33^{\circ} 47^{\prime} 40^{\prime \prime} \mathrm{N}$ & $116^{\circ} 25^{\prime} 22^{\prime \prime} \mathrm{W}$ & LP & 178 \\
\hline $4 \mathrm{~S} / 5 \mathrm{E}-26 \mathrm{~K} 1$ & $400-820$ & 340 & $33^{\circ} 47^{\prime} 36^{\prime \prime} \mathrm{N}$ & $116^{\circ} 25^{\prime} 50^{\prime \prime} \mathrm{W}$ & LP & 178 \\
\hline $4 \mathrm{~S} / 5 \mathrm{E}-27 \mathrm{E} 1$ & 344-392 & 313 & $33^{\circ} 47^{\prime} 48^{\prime \prime} \mathrm{N}$ & $116^{\circ} 27^{\prime} 25^{\prime \prime} \mathrm{W}$ & $\mathrm{L}$ & 167 \\
\hline $4 \mathrm{~S} / 5 \mathrm{E}-27 \mathrm{E} 2$ & $456-835$ & 315 & $33^{\circ} 47^{\prime} 48^{\prime \prime} \mathrm{N}$ & $116^{\circ} 27^{\prime} 25^{\prime \prime} \mathrm{W}$ & LP & 167 \\
\hline $4 \mathrm{~S} / 5 \mathrm{E}-27 \mathrm{~L} 1$ & -- & 300 & $33^{\circ} 47^{\prime} 32^{\prime \prime} \mathrm{N}$ & $116^{\circ} 27^{\prime} 35^{\prime \prime} \mathrm{W}$ & $\mathrm{P}$ & 167 \\
\hline $4 S / 5 E-28 F 1$ & $500-925$ & 322 & $33^{\circ} 47^{\prime} 49^{\prime \prime} \mathrm{N}$ & $116^{\circ} 28^{\prime} 07^{\prime \prime} \mathrm{W}$ & LP & 167 \\
\hline $4 \mathrm{~S} / 5 \mathrm{E}-28 \mathrm{~F} 2$ & $500-925$ & 312 & $33^{\circ} 47^{\prime} 41^{\prime \prime} \mathrm{N}$ & $116^{\circ} 28^{\prime} 08^{\prime \prime} \mathrm{W}$ & LP & 166 \\
\hline $4 S^{\prime} / 5 \mathrm{E}-28 \mathrm{M} 1$ & -- & 310 & $33^{\circ} 47^{\prime} 33^{\prime \prime} \mathrm{N}$ & $116^{\circ} 28^{\prime} 21^{\prime \prime} \mathrm{W}$ & $\mathrm{P}$ & 166 \\
\hline 4S'/5E-29A1 & $600-1,000$ & 327 & $33^{\circ} 48^{\prime} 03^{\prime \prime} \mathrm{N}$ & $116^{\circ} 28^{\prime} 33^{\prime \prime} \mathrm{W}$ & LPQ & 157 \\
\hline $4 \mathrm{~S} / 5 \mathrm{E}-29 \mathrm{~F} 1$ & $100-407$ & 338 & $33^{\circ} 47^{\prime} 50^{\prime \prime} \mathrm{N}$ & $116^{\circ} 29^{\prime} 07^{\prime \prime} \mathrm{W}$ & L & \\
\hline $4 \mathrm{~S} / 5 \mathrm{E}-29 \mathrm{~F} 2$ & -. & 330 & $33^{\circ} 47^{\prime} 45^{\prime \prime} \mathrm{N}$ & $116^{\circ} 29^{\prime} 08^{\prime \prime} \mathrm{W}$ & $\mathbf{P}$ & 156 \\
\hline $4 \mathrm{~S} / 5 \mathrm{E}-29 \mathrm{H} 1$ & $590-990$ & 320 & $33^{\circ} 47^{\prime} 41^{\prime \prime} \mathrm{N}$ & $116^{\circ} 28^{\prime} 32^{\prime \prime} \mathrm{W}$ & LPO & 166 \\
\hline $4 S^{\prime} / 5 \mathrm{E}-29 \mathrm{~K} 1$ & -- & 325 & $33^{\circ} 47^{\prime} 32^{\prime \prime} \mathrm{N}$ & $116^{\circ} 28^{\prime} 59^{\prime \prime} \mathrm{W}$ & LP & 156 \\
\hline $4 S^{\prime} / 5 E-30 C 1$ & $300-440$ & 357 & $33^{\circ} 47^{\prime} 51^{\prime \prime} \mathrm{N}$ & $116^{\circ} 30^{\prime} 00^{\prime \prime} \mathrm{W}$ & LPQ & 156 \\
\hline $4 \mathrm{~S} / 5 \mathrm{E}-33 \mathrm{~B} 1$ & $268-288$ & 302 & $33^{\circ} 47^{\prime} 09^{\prime \prime} \mathrm{N}$ & $116^{\circ} 27^{\prime} 56^{\prime \prime} \mathrm{W}$ & L & \\
\hline $4 \mathrm{~S} / 5 \mathrm{E}-33 \mathrm{~B} 4$ & $429-843$ & 291 & $33^{\circ} 47^{\prime} 12^{\prime \prime} \mathrm{N}$ & $116^{\circ} 27^{\prime} 57^{\prime \prime} \mathrm{W}$ & LPQ & 166 \\
\hline $4 \mathrm{~S} / 5 \mathrm{E}-33 \mathrm{G} 1$ & $172-436$ & 294 & $33^{\circ} 46^{\prime} 57^{\prime \prime} \mathrm{N}$ & $116^{\circ} 27^{\prime} 57^{\prime \prime} \mathrm{W}$ & LQ & \\
\hline $4 S / 5 E-34 C 1$ & $240-500$ & 290 & $33^{\circ} 47^{\prime} 07^{\prime \prime} \mathrm{N}$ & $116^{\circ} 27^{\prime} 08^{\prime \prime} \mathrm{W}$ & LP & 176 \\
\hline $4 \mathrm{~S} / 5 \mathrm{E}-34 \mathrm{~J} 1$ & $240-500$ & 272 & $33^{\circ} 46^{\prime} 44^{\prime \prime} \mathrm{N}$ & $116^{\circ} 26^{\prime} 34^{\prime \prime} \mathrm{W}$ & LP & 176 \\
\hline $4 \mathrm{~S} / 5 \mathrm{E}-35 \mathrm{D} 2$ & $215-407$ & 268 & $33^{\circ} 47^{\prime} 06^{\prime \prime} \mathrm{N}$ & $116^{\circ} 26^{\prime} 22^{\prime \prime} \mathrm{W}$ & L & \\
\hline $4 \mathrm{~S} / 5 \mathrm{E}-35 \mathrm{E} 1$ & -- & 268 & $33^{\circ} 46^{\prime} 59^{\prime \prime} \mathrm{N}$ & $116^{\circ} 26^{\prime} 22^{\prime \prime} \mathrm{W}$ & LP & 177 \\
\hline $4 \mathrm{~S} / 5 \mathrm{E}-35 \mathrm{G} 3$ & -- & 262 & $33^{\circ} 46^{\prime} 55^{\prime \prime} \mathrm{N}$ & $116^{\circ} 25^{\prime} 48^{\prime \prime} \mathrm{W}$ & $\mathrm{L}$ & \\
\hline 4S'/5E-35G4 & $600-1,000$ & 262 & $33^{\circ} 46^{\prime} 55^{\prime \prime} \mathrm{N}$ & $116^{\circ} 25^{\prime} 50^{\prime \prime} \mathrm{W}$ & LP & 177 \\
\hline $4 S^{\prime} / 5 \mathrm{E}-35 \mathrm{Q} 1$ & $171-518$ & 250 & $33^{\circ} 46^{\prime} 23^{\prime \prime} \mathrm{N}$ & $116^{\circ} 25^{\prime} 50^{\prime \prime} \mathrm{W}$ & LP & 186 \\
\hline $4 \mathrm{~S} / 5 \mathrm{E}-35 \mathrm{Q} 2$ & $340-600$ & 255 & $33^{\circ} 46^{\prime} 29^{\prime \prime} \mathrm{N}$ & $116^{\circ} 25^{\prime} 42^{\prime \prime} \mathrm{W}$ & LP & 187 \\
\hline $4 \mathrm{~S} / 5 \mathrm{E}-36 \mathrm{D} 1$ & $\begin{array}{l}470-492 \\
658-678\end{array}$ & 320 & $33^{\circ} 47^{\prime} 07^{\prime \prime} \mathrm{N}$ & $116^{\circ} 25^{\prime} 13^{\prime \prime} \mathrm{W}$ & $\mathrm{L}$ & \\
\hline 4S/5E-36L1 & $408-840$ & 300 & $33^{\circ} 46^{\prime} 46^{\prime \prime} \mathrm{N}$ & $116^{\circ} 24^{\prime} 54^{\prime \prime} \mathrm{W}$ & LP & 187 \\
\hline $4 \mathrm{~S} / 5 \mathrm{E}-36 \mathrm{~L} 2$ & $252-650$ & 300 & $33^{\circ} 46^{\prime} 40^{\prime \prime} \mathrm{N}$ & $116^{\circ} 24^{\prime} 58^{\prime \prime} \mathrm{W}$ & LP & 187 \\
\hline $4 \mathrm{~S} / 5 \mathrm{E}-36 \mathrm{M} 1$ & $315-627$ & 257 & $33^{\circ} 51^{\prime} 52^{\prime \prime} \mathrm{N}$ & $116^{\circ} 31^{\prime} 32^{\prime \prime} \mathrm{W}$ & LP & 187 \\
\hline $4 \mathrm{~S} / 6 \mathrm{E}-08 \mathrm{~L} 1$ & -. & 378 & $33^{\circ} 50^{\prime} 17^{\prime \prime} \mathrm{N}$ & $116^{\circ} 22^{\prime} 44^{\prime \prime}$ W & $\mathrm{L}$ & \\
\hline 4S/6E-17R1 & $166-817$ & 215 & $33^{\circ} 49^{\prime} 02^{\prime \prime} \mathrm{N}$ & $116^{\circ} 22^{\prime} 23^{\prime \prime} \mathrm{W}$ & LP & 192 \\
\hline 4S'/6E-18P1 & $216-454$ & 232 & $33^{\circ} 49^{\prime} 08^{\prime \prime} \mathrm{N}$ & $116^{\circ} 24^{\prime} 00^{\prime \prime} \mathrm{W}$ & LP & 180 \\
\hline
\end{tabular}


Appendix 1: List and description of wells in data base--Continued

\begin{tabular}{|c|c|c|c|c|c|c|}
\hline $\begin{array}{c}\text { Well } \\
\text { number }\end{array}$ & $\begin{array}{c}\text { Perforated } \\
\text { interval (depth, in } \\
\text { feet below land } \\
\text { surface) }\end{array}$ & $\begin{array}{c}\text { Land-surface } \\
\text { altitude (feet } \\
\text { above or } \\
\text { below (-) sea } \\
\text { level) }\end{array}$ & Latitude & Longitude & Remarks & $\begin{array}{l}\text { Closest } \\
\text { model node } \\
\text { for pumped } \\
\text { wells (in } \\
\text { model area } \\
\text { only) }\end{array}$ \\
\hline $\begin{array}{l}\text { 4S/6E-18Q2 } \\
\text { 4S/6E-18Q5 }\end{array}$ & $\begin{array}{l}125-255 \\
480-560 \\
610-640 \\
670-700 \\
780-830 \\
930-990\end{array}$ & $\begin{array}{l}242 \\
240\end{array}$ & $\begin{array}{l}33^{\circ} 49^{\prime} 10^{\prime \prime} \mathrm{N} \\
33^{\circ} 49^{\prime} 10^{\prime \prime} \mathrm{N}\end{array}$ & $\begin{array}{l}116^{\circ} 23^{\prime} 31^{\prime \prime} \mathrm{W} \\
116^{\circ} 23^{\prime} 29^{\prime \prime} \mathrm{W}\end{array}$ & $\underset{\mathbf{L}}{\mathbf{L P}}$ & 190 \\
\hline $\begin{array}{l}\text { 4S/6E-18R1 } \\
4 \mathrm{~S} / 6 \mathrm{E}-19 J 2\end{array}$ & $\begin{array}{l}479-518 \\
171-500\end{array}$ & $\begin{array}{l}240 \\
212\end{array}$ & $\begin{array}{l}33^{\circ} 49^{\prime} 09^{\prime \prime} \mathrm{N} \\
33^{\circ} 48^{\prime} 31^{\prime \prime} \mathrm{N}\end{array}$ & $\begin{array}{l}116^{\circ} 23^{\prime} 20^{\prime \prime} \mathrm{W} \\
116^{\circ} 23^{\prime} 28^{\prime \prime} \mathrm{W}\end{array}$ & $\underset{\mathrm{LP}}{\mathrm{LP}}$ & 190 \\
\hline $4 S / 6 E-20 A 1$ & $178-844$ & 205 & $33^{\circ} 48^{\prime} 46^{\prime \prime} \mathrm{N}$ & $116^{\circ} 22^{\prime} 28^{\prime \prime} \mathrm{W}$ & $\bar{L}$ & \\
\hline $4 S^{\prime} / 6 \mathrm{E}-20 \mathrm{C} 1$ & -- & 220 & $33^{\circ} 48^{\prime} 53^{\prime \prime} \mathrm{N}$ & $116^{\circ} 22^{\prime} 50^{\prime \prime} \mathrm{W}$ & $\mathbf{P}$ & 190 \\
\hline $4 S^{\prime} / 6 \mathrm{E}-20 \mathrm{~L} 1$ & $530-900$ & 198 & $33^{\circ} 48^{\prime} 21^{\prime \prime} \mathrm{N}$ & $116^{\circ} 22^{\prime} 51^{\prime \prime} \mathrm{W}$ & $\mathbf{L P}$ & 200 \\
\hline $4 \mathrm{~S} / 6 \mathrm{E}-20 \mathrm{M} 1$ & $460-960$ & 205 & $33^{\circ} 48^{\prime} 31^{\prime \prime} \mathrm{N}$ & $116^{\circ} 23^{\prime} 10^{\prime \prime} \mathrm{W}$ & LP & 190 \\
\hline $4 S / 6 E-22 C 1$ & $\begin{array}{l}208-211 \\
272-304\end{array}$ & 218 & $33^{\circ} 48^{\prime} 59^{\prime \prime} \mathrm{N}$ & $116^{\circ} 20^{\prime} 45^{\prime \prime} \mathrm{W}$ & LP & 210 \\
\hline $4 \mathrm{~S} / 6 \mathrm{E}-22 \mathrm{C} 2$ & $\begin{array}{l}189-213 \\
233-305\end{array}$ & 219 & $33^{\circ} 48^{\prime} 59^{\prime \prime} \mathrm{N}$ & $116^{\circ} 20^{\prime} 41^{\prime \prime} \mathrm{W}$ & L & \\
\hline $4 \mathrm{~S} / 6 \mathrm{E}-22 \mathrm{~J} 1$ & -- & 230 & $33^{\circ} 48^{\prime} 32^{\prime \prime} \mathrm{N}$ & $116^{\circ} 20^{\prime} 08^{\prime \prime} \mathrm{W}$ & L & \\
\hline $4 \mathrm{~S} / 6 \mathrm{E}-22 \mathrm{~K} 1$ & $150-495$ & 215 & $33^{\circ} 48^{\prime} 33^{\prime \prime} \mathrm{N}$ & $116^{\circ} 20^{\prime} 23^{\prime \prime} \mathrm{W}$ & $\bar{L}$ & \\
\hline $4 S / 6 E-25 C 2$ & $540-700$ & 193 & $33^{\circ} 47^{\prime} 55^{\prime \prime} \mathrm{N}$ & $116^{\circ} 18^{\prime} 47^{\prime \prime} \mathrm{W}$ & LP & 232 \\
\hline $4 S^{\prime} / 6 E-25 C 3$ & $540-700$ & 199 & $33^{\circ} 48^{\prime} 06^{\prime \prime} \mathrm{N}$ & $116^{\circ} 18^{\prime} 46^{\prime \prime} \mathrm{W}$ & LP & 221 \\
\hline $4 \mathrm{~S} / 6 \mathrm{E}-25 \mathrm{~J} 2$ & $110-340$ & 158 & $33^{\circ} 47^{\prime} 39^{\prime \prime} \mathrm{N}$ & $116^{\circ} 18^{\prime} 03^{\prime \prime} \mathrm{W}$ & LP & 232 \\
\hline $4 \mathrm{~S} / 6 \mathrm{E}-25 \mathrm{R} 1$ & $110-340$ & 150 & $33^{\circ} 47^{\prime} 22^{\prime \prime} \mathrm{N}$ & $116^{\circ} 18^{\prime} 03^{\prime \prime} \mathrm{W}$ & $\mathbf{L P}$ & 232 \\
\hline $4 S / 6 E-27 N 1$ & $506-563$ & 165 & $33^{\circ} 47^{\prime} 27^{\prime \prime} \mathrm{N}$ & $116^{\circ} 21^{\prime} 06^{\prime \prime} \mathrm{W}$ & L & \\
\hline $4 S / 6 E-28 A 2$ & $180-750$ & 175 & $33^{\circ} 48^{\prime} 01^{\prime \prime} \mathrm{N}$ & $116^{\circ} 21^{\prime} 18^{\prime \prime} \mathrm{W}$ & L & \\
\hline $4 \mathrm{~S} / 6 \mathrm{E}-28 \mathrm{E} 1$ & $106-452$ & 177 & $33^{\circ} 47^{\prime} 53^{\prime \prime} \mathrm{N}$ & $116^{\circ} 22^{\prime} 12^{\prime \prime} \mathrm{W}$ & LP & 200 \\
\hline $4 \mathrm{~S} / 6 \mathrm{E}-28 \mathrm{E} 3$ & $460-880$ & 177 & $33^{\circ} 47^{\prime} 55^{\prime \prime} \mathrm{N}$ & $116^{\circ} 22^{\prime} 10^{\prime \prime} \mathrm{W}$ & LP & 200 \\
\hline $4 S / 6 E-28 G 2$ & $106-822$ & 168 & $33^{\circ} 47^{\prime} 41^{\prime \prime} \mathrm{N}$ & $116^{\circ} 21^{\prime} 29^{\prime \prime} \mathrm{W}$ & LP & 209 \\
\hline $4 \mathrm{~S} / 6 \mathrm{E}-28 \mathrm{H} 1$ & $192-980$ & 167 & $33^{\circ} 47^{\prime} 43^{\prime \prime} \mathrm{N}$ & $116^{\circ} 21^{\prime} 12^{\prime \prime} \mathrm{W}$ & LP & 209 \\
\hline $4 \mathrm{~S} / 6 \mathrm{E}-28 \mathrm{~J} 2$ & -- & 166 & $33^{\circ} 47^{\prime} 28^{\prime \prime} \mathrm{N}$ & $116^{\circ} 21^{\prime} 21^{\prime \prime} \mathrm{W}$ & $\mathrm{L}$ & \\
\hline $4 \mathrm{~S} / 6 \mathrm{E}-28 \mathrm{~J} 3$ & $157-600$ & 164 & $33^{\circ} 47^{\prime} 29^{\prime \prime} \mathrm{N}$ & $116^{\circ} 21^{\prime} 11^{\prime \prime} \mathrm{W}$ & LP & 209 \\
\hline $4 S^{\prime} / 6 \mathrm{E}-28 J 7$ & $\begin{array}{l}500-680 \\
730-780 \\
810-880\end{array}$ & 165 & $33^{\circ} 47^{\prime} 34^{\prime \prime} \mathrm{N}$ & $116^{\circ} 21^{\prime} 10^{\prime \prime} \mathrm{W}$ & LP & 209 \\
\hline & $900-1,080$ & & & & & \\
\hline $4 \mathrm{~S} / 6 \mathrm{E}-28 \mathrm{~K} 4$ & $496-796$ & 168 & $33^{\circ} 47^{\prime} 40^{\prime \prime} \mathrm{N}$ & $116^{\circ} 21^{\prime} 39^{\prime \prime} \mathrm{W}$ & LP & 209 \\
\hline $4 \mathrm{~S} / 6 \mathrm{E}-29 \mathrm{~A} 1$ & $178-844$ & 179 & $33^{\circ} 48^{\prime} 05^{\prime \prime} \mathrm{N}$ & $116^{\circ} 22^{\prime} 13^{\prime \prime} \mathrm{W}$ & $\mathrm{L}$ & \\
\hline $4 S / 6 E-34 A 1$ & $300-1,015$ & 158 & $33^{\circ} 47^{\prime} 13^{\prime \prime} \mathrm{N}$ & $116^{\circ} 20^{\prime} 22^{\prime \prime} \mathrm{W}$ & LP & 220 \\
\hline $4 S / 6 E-34 C 1$ & $108-495$ & 163 & $33^{\circ} 47^{\prime} 13^{\prime \prime} \mathrm{N}$ & $116^{\circ} 20^{\prime} 49^{\prime \prime} \mathrm{W}$ & $\mathrm{P}$ & 220 \\
\hline 4S/6E-34D1 & -- & 162 & $33^{\circ} 47^{\prime} 13^{\prime \prime} \mathrm{N}$ & $116^{\circ} 21^{\prime} 08^{\prime \prime} \mathrm{W}$ & $\mathrm{L}$ & \\
\hline $4 S / 6 E-34 D 2$ & $312-880$ & 162 & $33^{\circ} 47^{\prime} 09^{\prime \prime} \mathrm{N}$ & $116^{\circ} 20^{\prime} 56^{\prime \prime} \mathrm{W}$ & LP & 220 \\
\hline $4 \mathrm{~S} / 6 \mathrm{E}-34 \mathrm{~F} 1$ & -- & 161 & $33^{\circ} 46^{\prime} 59^{\prime \prime} \mathrm{N}$ & $116^{\circ} 20^{\prime} 39^{\prime \prime} \mathrm{W}$ & L & \\
\hline $4 \mathrm{~S} / 6 \mathrm{E}-34 \mathrm{~K} 1$ & -- & 158 & $33^{\circ} 46^{\prime} 47^{\prime \prime} \mathrm{N}$ & $116^{\circ} 20^{\prime} 23^{\prime \prime} \mathrm{W}$ & LP & 220 \\
\hline $4 \mathrm{~S} / 6 \mathrm{E}-34 \mathrm{~K} 2$ & $716-1,150$ & 161 & $33^{\circ} 46^{\prime} 41^{\prime \prime} \mathrm{N}$ & $116^{\circ} 20^{\prime} 36^{\prime \prime} \mathrm{W}$ & LP & 219 \\
\hline $4 S / 6 E-34 L 1$ & $168-805$ & 165 & $33^{\circ} 46^{\prime} 47^{\prime \prime} \mathrm{N}$ & $116^{\circ} 20^{\prime} 44^{\prime \prime} \mathrm{W}$ & LP & 219 \\
\hline $4 S / 6 E-34 M 1$ & $450-1,000$ & 165 & $33^{\circ} 46^{\prime} 47^{\prime \prime} \mathrm{N}$ & $116^{\circ} 21^{\prime} 06^{\prime \prime} \mathrm{W}$ & LP & 219 \\
\hline $4 \mathrm{~S} / 6 \mathrm{E}-34 \mathrm{Q} 1$ & $171-602$ & 168 & $33^{\circ} 46^{\prime} 33^{\prime \prime} \mathrm{N}$ & $116^{\circ} 20^{\prime} 36^{\prime \prime} \mathrm{W}$ & $\mathrm{L}$ & \\
\hline $4 \mathrm{~S} / 6 \mathrm{E}-35 \mathrm{P} 1$ & $500-600$ & 150 & $33^{\circ} 46^{\prime} 27^{\prime \prime} \mathrm{N}$ & $116^{\circ} 19^{\prime} 45^{\prime \prime} \mathrm{W}$ & L & \\
\hline $4 \mathrm{~S} / 7 \mathrm{E}-14 \mathrm{E} 1$ & $382-520$ & 1,100 & $33^{\circ} 49^{\prime} 28^{\prime \prime} \mathrm{N}$ & $116^{\circ} 13^{\prime} 42^{\prime \prime} \mathrm{W}$ & $\mathbf{L}$ & \\
\hline 4S/7E-29E1 & $330-700$ & 118 & $33^{\circ} 47^{\prime} 41^{\prime \prime} \mathrm{N}$ & $116^{\circ} 16^{\prime} 49^{\prime \prime} \mathrm{W}$ & LP & 241 \\
\hline 4S/7E-29M1 & $570-680$ & 107 & $33^{\circ} 47^{\prime} 27^{\prime \prime} \mathrm{N}$ & $116^{\circ} 16^{\prime} 52^{\prime \prime} \mathrm{W}$ & LP & 241 \\
\hline $4 S / 7 E-29 N 2$ & $118-263$ & 103 & $33^{\circ} 47^{\prime} 16^{\prime \prime} \mathrm{N}$ & $116^{\circ} 16^{\prime} 52^{\prime \prime} \mathrm{W}$ & LP & 240 \\
\hline $4 S / 7 E-30 E 3$ & $180-360$ & 161 & $33^{\circ} 47^{\prime} 47^{\prime \prime} \mathrm{N}$ & $116^{\circ} 17^{\prime} 59^{\prime \prime} \mathrm{W}$ & LP & 232 \\
\hline $4 S / 7 E-30 L 1$ & $200-568$ & 150 & $33^{\circ} 47^{\prime} 37^{\prime \prime} \mathrm{N}$ & $116^{\circ} 17^{\prime} 44^{\prime \prime} \mathrm{W}$ & LP & 232 \\
\hline 4S/7E-30M1 & -- & 158 & $33^{\circ} 47^{\prime} 37^{\prime \prime} \mathrm{N}$ & $116^{\circ} 17^{\prime} 58^{\prime \prime} \mathrm{W}$ & $\mathbf{L}$ & \\
\hline
\end{tabular}


Appendix 1: List and description of wells in data base--Continued

\begin{tabular}{|c|c|c|c|c|c|c|}
\hline $\begin{array}{l}\text { Well } \\
\text { number }\end{array}$ & $\begin{array}{c}\text { Perforated } \\
\text { interval (depth, in } \\
\text { feet below land } \\
\text { surface) }\end{array}$ & $\begin{array}{l}\text { Land-surface } \\
\text { altitude (feet } \\
\text { above or } \\
\text { below (-) sea } \\
\text { level) }\end{array}$ & Latitude & Longitude & Remarks & $\begin{array}{c}\text { Closest } \\
\text { model node } \\
\text { for pumped } \\
\text { wells (in } \\
\text { model area } \\
\text { only) }\end{array}$ \\
\hline 4S/7E-30M2 & -- & 148 & $33^{\circ} 47^{\prime} 38^{\prime \prime} \mathrm{N}$ & $116^{\circ} 17^{\prime} 51^{\prime \prime} \mathrm{W}$ & LP & 232 \\
\hline 4S/7E-30M6 & -- & 150 & $33^{\circ} 47^{\prime} 32^{\prime \prime} \mathrm{N}$ & $116^{\circ} 17^{\prime} 49^{\prime \prime} \mathrm{W}$ & LP & 232 \\
\hline 4S/7E-30P1 & $148-360$ & 140 & $33^{\circ} 47^{\prime} 24^{\prime \prime} \mathrm{N}$ & $116^{\circ} 17^{\prime} 41^{\prime \prime} \mathrm{W}$ & LP & 232 \\
\hline $4 \mathrm{~S} / 7 \mathrm{E}-31 \mathrm{Q} 3$ & $\begin{array}{l}236-356 \\
590-710\end{array}$ & 97 & $33^{\circ} 46^{\prime} 32^{\prime \prime} \mathrm{N}$ & $116^{\circ} 17^{\prime} 26^{\prime \prime} \mathrm{W}$ & LP & 240 \\
\hline 4S/7E-32F2 & -- & 80 & $33^{\circ} 46^{\prime} 55^{\prime \prime} \mathrm{N}$ & $116^{\circ} 16^{\prime} 34^{\prime \prime} \mathrm{W}$ & $\mathbf{P}$ & 240 \\
\hline $4 \mathrm{~S} / 7 \mathrm{E}-32 \mathrm{G} 2$ & $\begin{array}{l}280-320 \\
380-400\end{array}$ & 73 & $33^{\circ} 46^{\prime} 50^{\prime \prime} \mathrm{N}$ & $116^{\circ} 16^{\prime} 20^{\prime \prime} \mathrm{W}$ & L & \\
\hline 4S/7E-32N2 & $195-462$ & 73 & $33^{\circ} 46^{\prime} 25^{\prime \prime} \mathrm{N}$ & $116^{\circ} 16^{\prime} 54^{\prime \prime} \mathrm{W}$ & LP & 240 \\
\hline 4S/7E-33N1 & $100-412$ & 54 & $33^{\circ} 46^{\prime} 31^{\prime \prime} \mathrm{N}$ & $116^{\circ} 15^{\prime} 51^{\prime \prime} \mathrm{W}$ & $\mathrm{L}$ & \\
\hline $4 S^{\prime} / 7 E-33 Q 1$ & $240-460$ & 48 & $33^{\circ} 46^{\prime} 24^{\prime \prime} \mathrm{N}$ & $116^{\circ} 15^{\prime} 22^{\prime \prime} \mathrm{W}$ & $\mathrm{L}$ & \\
\hline $4 \mathrm{~S} / 8 \mathrm{E}-31 \mathrm{R} 2$ & -- & 280 & $33^{\circ} 46^{\prime} 27^{\prime \prime} \mathrm{N}$ & $116^{\circ} 10^{\prime} 47^{\prime \prime} \mathrm{W}$ & $\mathrm{L}$ & \\
\hline $5 \mathrm{~S} / 4 \mathrm{E}-02 \mathrm{G} 1$ & $300-500$ & 580 & $33^{\circ} 46^{\prime} 05^{\prime \prime} \mathrm{N}$ & $116^{\circ} 32^{\prime} 14^{\prime \prime} \mathrm{W}$ & $\mathrm{L}$ & \\
\hline $5 \mathrm{~S} / 5 \mathrm{E}-01 \mathrm{~B} 1$ & $420-800$ & 247 & $33^{\circ} 46^{\prime} 10^{\prime \prime} \mathrm{N}$ & $116^{\circ} 24^{\prime} 54^{\prime \prime} \mathrm{W}$ & LP & 187 \\
\hline $5 \mathrm{~S} / 5 \mathrm{E}-01 \mathrm{C} 1$ & $216-350$ & 244 & $33^{\circ} 46^{\prime} 09^{\prime \prime} \mathrm{N}$ & $116^{\circ} 24^{\prime} 59^{\prime \prime} \mathrm{W}$ & $\mathrm{L}$ & \\
\hline $5 \mathrm{~S} / 5 \mathrm{E}-01 \mathrm{D} 2$ & -- & 250 & $33^{\circ} 46^{\prime} 16^{\prime \prime} \mathrm{N}$ & $116^{\circ} 25^{\prime} 22^{\prime \prime} \mathrm{W}$ & $\overline{\mathrm{L}}$ & \\
\hline $5 \mathrm{~S} / 5 \mathrm{E}-01 \mathrm{D} 3$ & -- & 250 & $33^{\circ} 46^{\prime} 14^{\prime \prime} \mathrm{N}$ & $116^{\circ} 25^{\prime} 21^{\prime \prime} \mathrm{W}$ & LP & 187 \\
\hline $5 \mathrm{~S} / 5 \mathrm{E}-01 \mathrm{~L} 5$ & $500-740$ & 242 & $33^{\circ} 45^{\prime} 43^{\prime \prime} \mathrm{N}$ & $116^{\circ} 25^{\prime} 10^{\prime \prime} \mathrm{W}$ & LP & 187 \\
\hline $5 \mathrm{~S} / 5 \mathrm{E}-01 \mathrm{M} 4$ & $150-502$ & 250 & $33^{\circ} 45^{\prime} 54^{\prime \prime} \mathrm{N}$ & $116^{\circ} 25^{\prime} 26^{\prime \prime} \mathrm{W}$ & LP & 186 \\
\hline $5 S^{\prime} / 5 E-01 N 1$ & -- & 235 & $33^{\circ} 45^{\prime} 29^{\prime \prime} \mathrm{N}$ & $116^{\circ} 25^{\prime} 19^{\prime \prime} \mathrm{W}$ & LP & 186 \\
\hline $5 \mathrm{~S} / 5 \mathrm{E}-01 \mathrm{P} 1$ & $108-192$ & 240 & $33^{\circ} 45^{\prime} 41^{\prime \prime} \mathrm{N}$ & $116^{\circ} 25^{\prime} 01^{\prime \prime} \mathrm{W}$ & $\mathrm{L}$ & \\
\hline $5 \mathrm{~S} / 5 \mathrm{E}-01 \mathrm{Q} 2$ & $400-835$ & 235 & $33^{\circ} 45^{\prime} 37^{\prime \prime} \mathrm{N}$ & $116^{\circ} 24^{\prime} 51^{\prime \prime} \mathrm{W}$ & LP & 186 \\
\hline $5 \mathrm{~S} / 5 \mathrm{E}-01 \mathrm{Q} 3$ & $400-820$ & 235 & $33^{\circ} 45^{\prime} 32^{\prime \prime} \mathrm{N}$ & $116^{\circ} 24^{\prime} 51^{\prime \prime} \mathrm{W}$ & LP & 186 \\
\hline $5 \mathrm{~S} / 5 \mathrm{E}-02 \mathrm{~A} 1$ & -- & 249 & $33^{\circ} 46^{\prime} 14^{\prime \prime} \mathrm{N}$ & $116^{\circ} 25^{\prime} 37^{\prime \prime} \mathrm{W}$ & LP & 186 \\
\hline $5 \mathrm{~S} / 5 \mathrm{E}-02 \mathrm{~B} 1$ & $450-600$ & 252 & $33^{\circ} 46^{\prime} 18^{\prime \prime} \mathrm{N}$ & $116^{\circ} 25^{\prime} 54^{\prime \prime} \mathrm{W}$ & LP & 186 \\
\hline $5 \mathrm{~S} / 5 \mathrm{E}-02 \mathrm{~F} 2$ & $250-495$ & 242 & $33^{\circ} 45^{\prime} 56^{\prime \prime} \mathrm{N}$ & $116^{\circ} 26^{\prime} 05^{\prime \prime} \mathrm{W}$ & $\mathbf{L}$ & 186 \\
\hline $5 \mathrm{~S} / 5 \mathrm{E}-02 \mathrm{H} 1$ & -- & 249 & $33^{\circ} 45^{\prime} 56^{\prime \prime} \mathrm{N}$ & $116^{\circ} 26^{\prime} 51^{\prime \prime} \mathrm{W}$ & LP & 186 \\
\hline $5 \mathrm{~S} / 5 \mathrm{E}-02 \mathrm{~L} 1$ & $\begin{array}{l}130-140 \\
200-220\end{array}$ & 252 & $33^{\circ} 45^{\prime} 52^{\prime \prime} \mathrm{N}$ & $116^{\circ} 26^{\prime} 14^{\prime \prime} \mathrm{W}$ & $\mathrm{L}$ & \\
\hline $5 \mathrm{~S} / 5 \mathrm{E}-02 \mathrm{Q} 1$ & $220-450$ & 239 & $33^{\circ} 45^{\prime} 40^{\prime \prime} \mathrm{N}$ & $116^{\circ} 25^{\prime} 47^{\prime \prime} \mathrm{W}$ & LP & 186 \\
\hline $5 \mathrm{~S} / 5 \mathrm{E}-03 \mathrm{~A} 1$ & $\begin{array}{l}110-130 \\
238-320\end{array}$ & 260 & $33^{\circ} 46^{\prime} 20^{\prime \prime} \mathrm{N}$ & $116^{\circ} 26^{\prime} 35^{\prime \prime} \mathrm{W}$ & $\mathrm{L}$ & \\
\hline 5S/5E-11A1 & -- & 234 & $33^{\circ} 45^{\prime} 20^{\prime \prime} \mathrm{N}$ & $116^{\circ} 25^{\prime} 40^{\prime \prime} \mathrm{W}$ & $\mathrm{L}$ & \\
\hline $5 \mathrm{~S} / 5 \mathrm{E}-11 \mathrm{~A} 3$ & $250-440$ & 239 & $33^{\circ} 45^{\prime} 26^{\prime \prime} \mathrm{N}$ & $116^{\circ} 25^{\prime} 32^{\prime \prime} \mathrm{W}$ & $\mathbf{L P}$ & 186 \\
\hline $5 S / 5 E-12 B 3$ & $410-800$ & 230 & $33^{\circ} 45^{\prime} 27^{\prime \prime} \mathrm{N}$ & $116^{\circ} 24^{\prime} 54^{\prime \prime} \mathrm{W}$ & LP & 186 \\
\hline $5 \mathrm{~S} / 5 \mathrm{E}-12 \mathrm{C} 1$ & $163-503$ & 233 & $33^{\circ} 45^{\prime} 24^{\prime \prime} \mathrm{N}$ & $116^{\circ} 25^{\prime} 01^{\prime \prime} \mathrm{W}$ & L & \\
\hline $5 \mathrm{~S} / 5 \mathrm{E}-12 \mathrm{C} 2$ & -- & 230 & $33^{\circ} 45^{\prime} 16^{\prime \prime} \mathrm{N}$ & $116^{\circ} 25^{\prime} 11^{\prime \prime} \mathrm{W}$ & $\mathbf{L}$ & \\
\hline $5 \mathrm{~S} / 5 \mathrm{E}-12 \mathrm{D} 1$ & $125-360$ & 239 & $33^{\circ} 45^{\prime} 26^{\prime \prime} \mathrm{N}$ & $116^{\circ} 25^{\prime} 25^{\prime \prime} \mathrm{W}$ & LP & 186 \\
\hline $5 \mathrm{~S} / 5 \mathrm{E}-12 \mathrm{D} 2$ & $278-498$ & 236 & $33^{\circ} 45^{\prime} 24^{\prime \prime} \mathrm{N}$ & $116^{\circ} 25^{\prime} 24^{\prime \prime} \mathrm{W}$ & LP & 186 \\
\hline $5 \mathrm{~S} / 5 \mathrm{E}-12 \mathrm{H} 2$ & $432-590$ & 220 & $33^{\circ} 45^{\prime} 07^{\prime \prime} \mathrm{N}$ & $116^{\circ} 24^{\prime} 27^{\prime \prime} \mathrm{W}$ & $\mathrm{L}$ & \\
\hline $5 S / 5 E-12 J 1$ & $400-880$ & 221 & $33^{\circ} 45^{\prime} 00^{\prime \prime} \mathrm{N}$ & $116^{\circ} 24^{\prime} 27^{\prime \prime} \mathrm{W}$ & LP & 196 \\
\hline $5 \mathrm{~S} / 5 \mathrm{E}-12 \mathrm{~L} 2$ & $208-461$ & 240 & $33^{\circ} 44^{\prime} 38^{\prime \prime} \mathrm{N}$ & $116^{\circ} 25^{\prime} 15^{\prime \prime} \mathrm{W}$ & LP & 195 \\
\hline $5 \mathrm{~S} / 5 \mathrm{E}-12 \mathrm{Q} 1$ & -- & 235 & $33^{\circ} 44^{\prime} 46^{\prime \prime} \mathrm{N}$ & $116^{\circ} 24^{\prime} 51^{\prime \prime} \mathrm{W}$ & LP & 195 \\
\hline $5 \mathrm{~S} / 6 \mathrm{E}-01 \mathrm{C} 1$ & $126-1,250$ & 125 & $33^{\circ} 46^{\prime} 21^{\prime \prime} \mathrm{N}$ & $116^{\circ} 18^{\prime} 47^{\prime \prime} \mathrm{W}$ & LP & 231 \\
\hline $5 \mathrm{~S} / 6 \mathrm{E}-01 \mathrm{D} 2$ & $\begin{array}{l}410-440 \\
510-626 \\
678-716 \\
774-801 \\
840-902 \\
920-980\end{array}$ & 132 & $33^{\circ} 46^{\prime} 19^{\prime \prime} \mathrm{N}$ & $116^{\circ} 19^{\prime} 05^{\prime \prime} \mathrm{W}$ & LP & 2.31 \\
\hline $5 S / 6 E-01 J 1$ & $500-730$ & 112 & $33^{\circ} 45^{\prime} 47^{\prime \prime} \mathrm{N}$ & $116^{\circ} 18^{\prime} 20^{\prime \prime} \mathrm{W}$ & LP & 239 \\
\hline $5 \mathrm{~S} / 6 \mathrm{E}-01 \mathrm{~J} 2$ & $320-620$ & 116 & $33^{\circ} 45^{\prime} 49^{\prime \prime} \mathrm{N}$ & $116^{\circ} 18^{\prime} 19^{\prime \prime} \mathrm{W}$ & LP & 239 \\
\hline $5 \mathrm{~S} / 6 \mathrm{E}-01 \mathrm{~K} 2$ & $481-601$ & 120 & $33^{\circ} 45^{\prime} 55^{\prime \prime} \mathrm{N}$ & $116^{\circ} 18^{\prime} 40^{\prime \prime} \mathrm{W}$ & LP & 239 \\
\hline $5 S / 6 E-02 A 1$ & $200-618$ & 140 & $33^{\circ} 46^{\prime} 18^{\prime \prime} \mathrm{N}$ & $116^{\circ} 19^{\prime} 14^{\prime \prime} \mathrm{W}$ & $\mathrm{L}$ & \\
\hline
\end{tabular}


Appendix 1: List and description of wells in data base--Continued

\begin{tabular}{|c|c|c|c|c|c|c|}
\hline $\begin{array}{l}\text { Well } \\
\text { number }\end{array}$ & $\begin{array}{c}\text { Perforated } \\
\text { interval (depth, in } \\
\text { feet below land } \\
\text { surface) }\end{array}$ & $\begin{array}{l}\text { Land-surface } \\
\text { altitude (feet } \\
\text { above or } \\
\text { below (-) sea } \\
\text { level) }\end{array}$ & Latitude & Longitude & Remarks & $\begin{array}{l}\text { Closest } \\
\text { model node } \\
\text { for pumped } \\
\text { wells (in } \\
\text { model area } \\
\text { only) }\end{array}$ \\
\hline $5 \mathrm{~S} / 6 \mathrm{E}-02 \mathrm{~A} 2$ & $450-650$ & 140 & $33^{\circ} 46^{\prime} 15^{\prime \prime} \mathrm{N}$ & $116^{\circ} 19^{\prime} 17^{\prime \prime} \mathrm{W}$ & $\mathbf{L}$ & \\
\hline $5 S / 6 E-02 D 1$ & $350-1,250$ & 155 & $33^{\circ} 46^{\prime} 15^{\prime \prime} \mathrm{N}$ & $116^{\circ} 20^{\prime} 08^{\prime \prime} \mathrm{W}$ & LP & 230 \\
\hline $5 \mathrm{~S} / 6 \mathrm{E}-02 \mathrm{G} 1$ & $\begin{array}{c}470-600 \\
670-690 \\
710-770 \\
890-1,000\end{array}$ & 140 & $33^{\circ} 46^{\prime} 02^{\prime \prime} \mathrm{N}$ & $116^{\circ} 19^{\prime} 37^{\prime \prime} \mathrm{W}$ & LP & 230 \\
\hline $5 \mathrm{~S} / 6 \mathrm{E}-02 \mathrm{M} 1$ & $665-1,305$ & 170 & $33^{\circ} 45^{\prime} 44^{\prime \prime} \mathrm{N}$ & $116^{\circ} 19^{\prime} 57^{\prime \prime} \mathrm{W}$ & LP & 230 \\
\hline $5 S / 6 E-03 A 1$ & $\begin{array}{l}520-560 \\
610-680 \\
780-826 \\
906-980\end{array}$ & 164 & $33^{\circ} 46^{\prime} 21^{\prime \prime} \mathrm{N}$ & $116^{\circ} 20^{\prime} 28^{\prime \prime} \mathrm{W}$ & LP & 219 \\
\hline 5S/6E-03B1 & $480-1,040$ & 170 & $33^{\circ} 46^{\prime} 19^{\prime \prime} \mathrm{N}$ & $116^{\circ} 20^{\prime} 35^{\prime \prime} \mathrm{W}$ & L & \\
\hline $5 \mathrm{~S} / 6 \mathrm{E}-03 \mathrm{H} 1$ & $520-1,100$ & 195 & $33^{\circ} 46^{\prime} 05^{\prime \prime} \mathrm{N}$ & $116^{\circ} 20^{\prime} 30^{\prime \prime} \mathrm{W}$ & LP & 219 \\
\hline $5 \mathrm{~S} / 6 \mathrm{E}-03 \mathrm{~K} 1$ & $\begin{array}{c}710-770 \\
800-860 \\
910-990 \\
1,020-1,090\end{array}$ & 230 & $33^{\circ} 45^{\prime} 47^{\prime \prime} \mathrm{N}$ & $116^{\circ} 20^{\prime} 36^{\prime \prime} \mathrm{W}$ & LP & 218 \\
\hline 5S/6E-03L1 & -- & 240 & $33^{\circ} 45^{\prime} 48^{\prime \prime} \mathrm{N}$ & $116^{\circ} 20^{\prime} 55^{\prime \prime} \mathrm{W}$ & LP & 218 \\
\hline $5 \mathrm{~S} / 6 \mathrm{E}-03 \mathrm{~N} 1$ & $750-950$ & 260 & $33^{\circ} 45^{\prime} 39^{\prime \prime} \mathrm{N}$ & $116^{\circ} 21^{\prime} 14^{\prime \prime} \mathrm{W}$ & LP & 218 \\
\hline $5 \mathrm{~S} / 6 \mathrm{E}-03 \mathrm{P} 1$ & $570-970$ & 260 & $33^{\circ} 45^{\prime} 35^{\prime \prime} \mathrm{N}$ & $116^{\circ} 20^{\prime} 55^{\prime \prime} \mathrm{W}$ & LP & 218 \\
\hline $5 \mathrm{~S} / 6 \mathrm{E}-05 \mathrm{~A} 1$ & $520-1,220$ & 282 & $33^{\circ} 46^{\prime} 18^{\prime \prime} \mathrm{N}$ & $116^{\circ} 22^{\prime} 29^{\prime \prime} \mathrm{W}$ & LP & 207 \\
\hline $5 \mathrm{~S} / 6 \mathrm{E}-05 \mathrm{~B} 1$ & $850-1,045$ & 285 & $33^{\circ} 46^{\prime} 18^{\prime \prime} \mathrm{N}$ & $116^{\circ} 22^{\prime} 51^{\prime \prime} \mathrm{W}$ & LP & 198 \\
\hline $5 \mathrm{~S} / 6 \mathrm{E}-05 \mathrm{~K} 1$ & $1,036-1,300$ & 260 & $33^{\circ} 45^{\prime} 52^{\prime \prime} \mathrm{N}$ & $116^{\circ} 22^{\prime} 42^{\prime \prime} W$ & LP & 197 \\
\hline $5 \mathrm{~S} / 6 \mathrm{E}-05 \mathrm{M} 1$ & $540-1,040$ & 262 & $33^{\circ} 45^{\prime} 54^{\prime \prime} \mathrm{N}$ & $116^{\circ} 23^{\prime} 09^{\prime \prime} \mathrm{W}$ & LP & 197 \\
\hline $5 \mathrm{~S} / 6 \mathrm{E}-05 \mathrm{Q} 1$ & $\begin{array}{l}525-730 \\
800-890\end{array}$ & 250 & $33^{\circ} 45^{\prime} 36^{\prime \prime} \mathrm{N}$ & $116^{\circ} 22^{\prime} 45^{\prime \prime} \mathrm{W}$ & LP & 197 \\
\hline & $1,020-1,135$ & & & & & \\
\hline $\begin{array}{l}5 \mathrm{~S} / 6 \mathrm{E}-06 \mathrm{B3} \\
5 \mathrm{~S} / 6 \mathrm{E}-06 \mathrm{C1}\end{array}$ & $455-890$ & 290 & $33^{\circ} 46^{\prime} 17^{\prime \prime} \mathrm{N}$ & $116^{\circ} 23^{\prime} 47^{\prime \prime} \mathrm{W}$ & LP & 197 \\
\hline $5 S / 6 \mathrm{E}-06 \mathrm{C} 2$ & $\begin{array}{l}-- \\
--\end{array}$ & $\begin{array}{l}285 \\
284\end{array}$ & $\begin{array}{l}33^{\circ} 46^{\prime} 14^{\prime \prime} \mathrm{N} \\
33^{\circ} 46^{\prime} 14^{\prime \prime} \mathrm{N}\end{array}$ & $\begin{array}{l}116^{\circ} 24^{\prime} 22^{\prime \prime} \mathrm{W} \\
116^{\circ} 24^{\prime} 22^{\prime \prime} \mathrm{W}\end{array}$ & $\begin{array}{l}\text { LP } \\
\text { LP }\end{array}$ & $\begin{array}{l}187 \\
187\end{array}$ \\
\hline $5 \mathrm{~S} / 6 \mathrm{E}-06 \mathrm{M} 1$ & $420-820$ & 233 & $33^{\circ} 45^{\prime} 50^{\prime \prime} \mathrm{N}$ & $116^{\circ} 24^{\prime} 21^{\prime \prime} \mathrm{W}$ & LP & 187 \\
\hline $5 \mathrm{~S} / 6 \mathrm{E}-06 \mathrm{M} 2$ & $420-1,000$ & 230 & $33^{\circ} 45^{\prime} 50^{\prime \prime} \mathrm{N}$ & $116^{\circ} 24^{\prime} 21^{\prime \prime} \mathrm{W}$ & LP & 187 \\
\hline 5S/6E-06Q1 & $668-1,202$ & 220 & $33^{\circ} 45^{\prime} 31^{\prime \prime} \mathrm{N}$ & $116^{\circ} 23^{\prime} 53^{\prime \prime} \mathrm{W}$ & LP & 197 \\
\hline $5 \mathrm{~S} / 6 \mathrm{E}-07 \mathrm{~B} 1$ & -- & 215 & $33^{\circ} 45^{\prime} 22^{\prime \prime} \mathrm{N}$ & $116^{\circ} 23^{\prime} 47^{\prime \prime} \mathrm{W}$ & LP & 196 \\
\hline $5 \mathrm{~S} / 6 \mathrm{E}-07 \mathrm{C} 2$ & $320-500$ & 220 & $33^{\circ} 45^{\prime} 27^{\prime \prime} \mathrm{N}$ & $116^{\circ} 24^{\prime} 09^{\prime \prime} \mathrm{W}$ & LP & 196 \\
\hline 5S/6E-07J1 & $460-560$ & 210 & $33^{\circ} 44^{\prime} 50^{\prime \prime} \mathrm{N}$ & $116^{\circ} 23^{\prime} 38^{\prime \prime} \mathrm{W}$ & LP & 196 \\
\hline 5S/6E-07M1 & -- & 220 & $33^{\circ} 44^{\prime} 38^{\prime \prime} \mathrm{N}$ & $116^{\circ} 24^{\prime} 30^{\prime \prime} \mathrm{W}$ & LP & 195 \\
\hline $5 \mathrm{~S} / 6 \mathrm{E}-07 \mathrm{~N} 1$ & $144-200$ & 220 & $33^{\circ} 44^{\prime} 47^{\prime \prime} \mathrm{N}$ & $116^{\circ} 24^{\prime} 24^{\prime \prime} W$ & LP & 196 \\
\hline $5 S / 6 \mathrm{E}-07 \mathrm{Q} 2$ & $300-500$ & 210 & $33^{\circ} 44^{\prime} 48^{\prime \prime} \mathrm{N}$ & $116^{\circ} 23^{\prime} 46^{\prime \prime} \mathrm{W}$ & $\mathrm{L}$ & \\
\hline $5 \mathrm{~S} / 6 \mathrm{E}-07 \mathrm{Q} 3$ & $380-500$ & 210 & $33^{\circ} 44^{\prime} 47^{\prime \prime} \mathrm{N}$ & $116^{\circ} 23^{\prime} 53^{\prime \prime} \mathrm{W}$ & $\mathrm{L}$ & \\
\hline $5 S / 6 E-07 R 1$ & $364-604$ & 206 & $33^{\circ} 44^{\prime} 25^{\prime \prime} \mathrm{N}$ & $116^{\circ} 23^{\prime} 46^{\prime \prime} \mathrm{W}$ & LP & 205 \\
\hline $5 S / 6 \mathrm{E}-08 \mathrm{M} 2$ & $280-520$ & 205 & $33^{\circ} 44^{\prime} 50^{\prime \prime} \mathrm{N}$ & $116^{\circ} 23^{\prime} 21^{\prime \prime} \mathrm{W}$ & LP & 205 \\
\hline $5 \mathrm{~S} / 6 \mathrm{E}-08 \mathrm{M} 3$ & $650-920$ & 205 & $33^{\circ} 44^{\prime} 38^{\prime \prime} \mathrm{N}$ & $116^{\circ} 23^{\prime} 32^{\prime \prime} \mathrm{W}$ & LP & 205 \\
\hline $5 \mathrm{~S} / 6 \mathrm{E}-08 \mathrm{~N} 2$ & $450-780$ & 210 & $33^{\circ} 44^{\prime} 36^{\prime \prime} \mathrm{N}$ & $116^{\circ} 23^{\prime} 24^{\prime \prime} \mathrm{W}$ & LP & 205 \\
\hline $5 \mathrm{~S} / 6 \mathrm{E}-08 \mathrm{~N} 3$ & $390-690$ & 200 & $33^{\circ} 44^{\prime} 47^{\prime \prime} \mathrm{N}$ & $116^{\circ} 23^{\prime} 19^{\prime \prime} \mathrm{W}$ & LP & 205 \\
\hline 5S/6E-08P1 & -- & 200 & $33^{\circ} 44^{\prime} 48^{\prime \prime} \mathrm{N}$ & $116^{\circ} 22^{\prime} 55^{\prime \prime} \mathrm{W}$ & $\mathrm{L}$ & \\
\hline 5S/6E-08Q1 & -- & 195 & $33^{\circ} 44^{\prime} 20^{\prime \prime} \mathrm{N}$ & $116^{\circ} 23^{\prime} 08^{\prime \prime} \mathrm{W}$ & LP & 205 \\
\hline $5 \mathrm{~S} / 6 \mathrm{E}-09 \mathrm{~B} 1$ & -- & 262 & $33^{\circ} 45^{\prime} 17^{\prime \prime} \mathrm{N}$ & $116^{\circ} 21^{\prime} 34^{\prime \prime} \mathrm{W}$ & LP & 218 \\
\hline 5S/6E-09E1 & $\begin{array}{l}550-580 \\
630-730\end{array}$ & 285 & $33^{\circ} 45^{\prime} 09^{\prime \prime} \mathrm{N}$ & $116^{\circ} 22^{\prime} 13^{\prime \prime} \mathrm{W}$ & $\mathrm{L}$ & \\
\hline 5S/6E-09F1 & $\begin{array}{l}450-490 \\
530-670 \\
770-830\end{array}$ & 280 & $33^{\circ} 45^{\prime} 08^{\prime \prime} \mathrm{N}$ & $116^{\circ} 21^{\prime} 58^{\prime \prime} \mathrm{W}$ & L & \\
\hline 5S/6E-09M1 & -- & 200 & $33^{\circ} 44^{\prime} 54^{\prime \prime} \mathrm{N}$ & $116^{\circ} 22^{\prime} 14^{\prime \prime} \mathrm{W}$ & & \\
\hline
\end{tabular}


Appendix 1: List and description of wells in data base--Continued

\begin{tabular}{|c|c|c|c|c|c|c|}
\hline $\begin{array}{l}\text { Well } \\
\text { number }\end{array}$ & $\begin{array}{c}\text { Perforated } \\
\text { interval (depth, in } \\
\text { feet below land } \\
\text { surface) }\end{array}$ & $\begin{array}{l}\text { Land-surface } \\
\text { altitude (feet } \\
\text { above or } \\
\text { below (-) sea } \\
\text { level) }\end{array}$ & Latitude & Longitude & Remarks & $\begin{array}{c}\text { Closest } \\
\text { model node } \\
\text { for pumped } \\
\text { wells (in } \\
\text { model area } \\
\text { only) }\end{array}$ \\
\hline 5S/6E-09Q1 & $740-1,010$ & 200 & $33^{\circ} 44^{\prime} 39^{\prime \prime} \mathrm{N}$ & $116^{\circ} 21^{\prime} 48^{\prime \prime} \mathrm{W}$ & LP & 217 \\
\hline $5 \mathrm{~S} / 6 \mathrm{E}-10 \mathrm{D} 1$ & $530-1,030$ & 260 & $33^{\circ} 45^{\prime} 24^{\prime \prime} \mathrm{N}$ & $116^{\circ} 21^{\prime} 15^{\prime \prime} \mathrm{W}$ & LP & 218 \\
\hline $5 \mathrm{~S} / 6 \mathrm{E}-10 \mathrm{D} 2$ & -- & 260 & $33^{\circ} 45^{\prime} 24^{\prime \prime} \mathrm{N}$ & $116^{\circ} 20^{\prime} 21^{\prime \prime} \mathrm{W}$ & LP & 229 \\
\hline $5 \mathrm{~S} / 6 \mathrm{E}-10 \mathrm{E} 1$ & $\begin{array}{l}480-670 \\
820-925\end{array}$ & 265 & $33^{\circ} 44^{\prime} 54^{\prime \prime} \mathrm{N}$ & $116^{\circ} 22^{\prime} 14^{\prime \prime} \mathrm{W}$ & LP & 218 \\
\hline $5 S / 6 E-12 G 1$ & $\begin{array}{l}192-272 \\
472-604\end{array}$ & 121 & $33^{\circ} 45^{\prime} 15^{\prime \prime} \mathrm{N}$ & $116^{\circ} 18^{\prime} 39^{\prime \prime} \mathrm{W}$ & $\mathbf{L}$ & \\
\hline 5S/6E-12M1 & $140-650$ & 158 & $33^{\circ} 45^{\prime} 00^{\prime \prime} \mathrm{N}$ & $116^{\circ} 19^{\prime} 11^{\prime \prime} \mathrm{W}$ & $\mathbf{L P}$ & 237 \\
\hline $5 \mathrm{~S} / 6 \mathrm{E}-12 \mathrm{M} 2$ & $200-500$ & 150 & $33^{\circ} 45^{\prime} 00^{\prime \prime} \mathrm{N}$ & $116^{\circ} 19^{\prime} 05^{\prime \prime} \mathrm{W}$ & LP & 237 \\
\hline $5 \mathrm{~S} / 6 \mathrm{E}-12 \mathrm{~N} 1$ & $470-946$ & 178 & $33^{\circ} 44^{\prime} 36^{\prime \prime} \mathrm{N}$ & $116^{\circ} 19^{\prime} 11^{\prime \prime} \mathrm{W}$ & LP & 237 \\
\hline $5 \mathrm{~S} / 6 \mathrm{E}-12 \mathrm{Q} 1$ & -- & 160 & $33^{\circ} 44^{\prime} 41^{\prime \prime} \mathrm{N}$ & $116^{\circ} 18^{\prime} 34^{\prime \prime} \mathrm{W}$ & LP & 237 \\
\hline $5 \mathrm{~S} / 6 \mathrm{E}-12 \mathrm{Q} 2$ & -- & 160 & $33^{\circ} 44^{\prime} 41^{\prime \prime} \mathrm{N}$ & $116^{\circ} 18^{\prime} 34^{\prime \prime} \mathrm{W}$ & LP & 237 \\
\hline $5 \mathrm{~S} / 6 \mathrm{E}-13 \mathrm{~B} 1$ & $302-310$ & 145 & $33^{\circ} 44^{\prime} 28^{\prime \prime} \mathrm{N}$ & $116^{\circ} 18^{\prime} 30^{\prime \prime} \mathrm{W}$ & LP & 237 \\
\hline $5 S / 6 E-13 D 1$ & $\begin{array}{l}518-566 \\
614-878\end{array}$ & 169 & $33^{\circ} 44^{\prime} 25^{\prime \prime} \mathrm{N}$ & $116^{\circ} 18^{\prime} 59^{\prime \prime} \mathrm{W}$ & LP & 237 \\
\hline $5 \mathrm{~S} / 6 \mathrm{E}-13 \mathrm{M} 1$ & $\begin{array}{l}339-369 \\
387-400 \\
517-626 \\
740-900\end{array}$ & 197 & $33^{\circ} 44^{\prime} 08^{\prime \prime} \mathrm{N}$ & $116^{\circ} 19^{\prime} 10^{\prime \prime} \mathrm{W}$ & LP & 237 \\
\hline $5 S / 6 E-13 R 1$ & $470-650$ & 150 & $33^{\circ} 43^{\prime} 49^{\prime \prime} \mathrm{N}$ & $116^{\circ} 18^{\prime} 20^{\prime \prime} \mathrm{W}$ & LP & 244 \\
\hline $5 \mathrm{~S} / 6 \mathrm{E}-14 \mathrm{G} 1$ & $\begin{array}{l}520-600 \\
630-670\end{array}$ & 206 & $33^{\circ} 44^{\prime} 10^{\prime \prime} \mathrm{N}$ & $116^{\circ} 19^{\prime} 44^{\prime \prime} \mathrm{W}$ & LP & 228 \\
\hline $5 S / 6 E-14 G 2$ & -- & 210 & $33^{\circ} 44^{\prime} 14^{\prime \prime} \mathrm{N}$ & $116^{\circ} 19^{\prime} 38^{\prime \prime} \mathrm{W}$ & LP & 237 \\
\hline $5 S / 6 E-14 Q 1$ & $\begin{array}{l}440-600 \\
706-810\end{array}$ & 158 & $33^{\circ} 43^{\prime} 45^{\prime \prime} \mathrm{N}$ & $116^{\circ} 19^{\prime} 43^{\prime \prime} \mathrm{W}$ & LP & 228 \\
\hline $5 S / 6 E-16 A 1$ & $360-560$ & 181 & $33^{\circ} 44^{\prime} 22^{\prime \prime} \mathrm{N}$ & $116^{\circ} 21^{\prime} 23^{\prime \prime} \mathrm{W}$ & LP & 217 \\
\hline $5 S / 6 E-16 A 2$ & $\begin{array}{l}445-530 \\
730-965\end{array}$ & 190 & $33^{\circ} 44^{\prime} 22^{\prime \prime} \mathrm{N}$ & $116^{\circ} 21^{\prime} 34^{\prime \prime} \mathrm{W}$ & LP & 217 \\
\hline $5 \mathrm{~S} / 6 \mathrm{E}-16 \mathrm{C} 1$ & -- & 200 & $33^{\circ} 44^{\prime} 28^{\prime \prime} \mathrm{N}$ & $116^{\circ} 22^{\prime} 00^{\prime \prime} \mathrm{W}$ & LP & 217 \\
\hline $5 \mathrm{~S} / 6 \mathrm{E}-16 \mathrm{H} 1$ & $336-552$ & 165 & $33^{\circ} 44^{\prime} 14^{\prime \prime} \mathrm{N}$ & $116^{\circ} 21^{\prime} 21^{\prime \prime} \mathrm{W}$ & LP & 217 \\
\hline $5 \mathrm{~S} / 6 \mathrm{E}-16 \mathrm{~K} 1$ & $302-366$ & 165 & $33^{\circ} 44^{\prime} 03^{\prime \prime} \mathrm{N}$ & $116^{\circ} 21^{\prime} 44^{\prime \prime} \mathrm{W}$ & $\mathbf{L P}$ & 216 \\
\hline $5 \mathrm{~S} / 6 \mathrm{E}-16 \mathrm{M} 1$ & $350-500$ & 179 & $33^{\circ} 44^{\prime} 04^{\prime \prime} \mathrm{N}$ & $116^{\circ} 22^{\prime} 17^{\prime \prime} \mathrm{W}$ & LP & 216 \\
\hline $5 \mathrm{~S} / 6 \mathrm{E}-16 \mathrm{~N} 2$ & $564-904$ & 180 & $33^{\circ} 43^{\prime} 44^{\prime \prime} \mathrm{N}$ & $116^{\circ} 22^{\prime} 18^{\prime \prime} \mathrm{W}$ & LP & 216 \\
\hline $5 \mathrm{~S} / 6 \mathrm{E}-16 \mathrm{Q} 1$ & $156-300$ & 165 & $33^{\circ} 43^{\prime} 51^{\prime \prime} \mathrm{N}$ & $116^{\circ} 21^{\prime} 40^{\prime \prime} \mathrm{W}$ & LP & 216 \\
\hline $5 S / 6 E-16 Q 2$ & $242-354$ & 165 & $33^{\circ} 43^{\prime} 48^{\prime \prime} \mathrm{N}$ & $116^{\circ} 21^{\prime} 47^{\prime \prime} \mathrm{W}$ & LP & 216 \\
\hline $5 \mathrm{~S} / 6 \mathrm{E}-17 \mathrm{~F} 1$ & $290-590$ & 198 & $33^{\circ} 44^{\prime} 13^{\prime \prime} \mathrm{N}$ & $116^{\circ} 23^{\prime} 09^{\prime \prime} \mathrm{W}$ & LP & 205 \\
\hline $5 \mathrm{~S} / 6 \mathrm{E}-17 \mathrm{G} 2$ & $550-890$ & 195 & $33^{\circ} 44^{\prime} 10^{\prime \prime} \mathrm{N}$ & $116^{\circ} 22^{\prime} 40^{\prime \prime} \mathrm{W}$ & LP & 205 \\
\hline $5 \mathrm{~S} / 6 \mathrm{E}-17 \mathrm{M} 2$ & $376-700$ & 195 & $33^{\circ} 44^{\prime} 04^{\prime \prime} \mathrm{N}$ & $116^{\circ} 23^{\prime} 19^{\prime \prime} \mathrm{W}$ & LP & 205 \\
\hline $5 \mathrm{~S} / 6 \mathrm{E}-17 \mathrm{P} 2$ & $420-700$ & 190 & $33^{\circ} 43^{\prime} 44^{\prime \prime} \mathrm{N}$ & $116^{\circ} 22^{\prime} 58^{\prime \prime} \mathrm{W}$ & LP & 216 \\
\hline $5 S / 6 E-18 C 2$ & $300-650$ & 220 & $33^{\circ} 44^{\prime} 23^{\prime \prime} \mathrm{N}$ & $116^{\circ} 24^{\prime} 09^{\prime \prime} \mathrm{W}$ & LP & 195 \\
\hline $5 \mathrm{~S} / 6 \mathrm{E}-18 \mathrm{C} 3$ & -- & 200 & $33^{\circ} 44^{\prime} 13^{\prime \prime} \mathrm{N}$ & $116^{\circ} 24^{\prime} 16^{\prime \prime} \mathrm{W}$ & LP & 204 \\
\hline $5 \mathrm{~S} / 6 \mathrm{E}-18 \mathrm{G} 1$ & $377-497$ & 200 & $33^{\circ} 44^{\prime} 11^{\prime \prime} \mathrm{N}$ & $116^{\circ} 23^{\prime} 54^{\prime \prime}$ W & LP & 204 \\
\hline $5 \mathrm{~S} / 6 \mathrm{E}-18 \mathrm{~L} 2$ & $317-509$ & 198 & $33^{\circ} 44^{\prime} 02^{\prime \prime} \mathrm{N}$ & $116^{\circ} 23^{\prime} 58^{\prime \prime} \mathrm{W}$ & LP & 204 \\
\hline 5S/6E-18R1 & $296-450$ & 193 & $33^{\circ} 43^{\prime} 55^{\prime \prime} \mathrm{N}$ & $116^{\circ} 23^{\prime} 27^{\prime \prime} \mathrm{W}$ & $\mathbf{L}$ & \\
\hline $5 \mathrm{~S} / 6 \mathrm{E}-18 \mathrm{R} 2$ & $425-800$ & 193 & $33^{\circ} 43^{\prime} 55^{\prime \prime} \mathrm{N}$ & $116^{\circ} 23^{\prime} 27^{\prime \prime} \mathrm{W}$ & LP & 204 \\
\hline $5 \mathrm{~S} / 6 \mathrm{E}-20 \mathrm{~A} 1$ & $435-522$ & 200 & $33^{\circ} 43^{\prime} 30^{\prime \prime} \mathrm{N}$ & $116^{\circ} 22^{\prime} 23^{\prime \prime} \mathrm{W}$ & $\overline{L P}$ & 216 \\
\hline $5 \mathrm{~S} / 6 \mathrm{E}-20 \mathrm{~F} 2$ & $420-497$ & 210 & $33^{\circ} 43^{\prime} 10^{\prime \prime} \mathrm{N}$ & $116^{\circ} 23^{\prime} 16^{\prime \prime} \mathrm{W}$ & LP & 215 \\
\hline $5 \mathrm{~S} / 6 \mathrm{E}-20 \mathrm{F3}$ & $625-925$ & 210 & $33^{\circ} 43^{\prime} 23^{\prime \prime} \mathrm{N}$ & $116^{\circ} 23^{\prime} 04^{\prime \prime} \mathrm{W}$ & LP & 215 \\
\hline $5 \mathrm{~S} / 6 \mathrm{E}-20 \mathrm{P} 1$ & $300-588$ & 267 & $33^{\circ} 43^{\prime} 02^{\prime \prime} \mathrm{N}$ & $116^{\circ} 23^{\prime} 02^{\prime \prime} \mathrm{W}$ & LP & 215 \\
\hline $5 S / 6 E-21 G 1$ & $167-237$ & 210 & $33^{\circ} 43^{\prime} 18^{\prime \prime} \mathrm{N}$ & $116^{\circ} 21^{\prime} 42^{\prime \prime} \mathrm{W}$ & LP & 216 \\
\hline $5 \mathrm{~S} / 6 \mathrm{E}-21 \mathrm{G} 3$ & $195-500$ & 195 & $33^{\circ} 43^{\prime} 24^{\prime \prime} \mathrm{N}$ & $116^{\circ} 21^{\prime} 45^{\prime \prime} \mathrm{W}$ & LP & 216 \\
\hline $5 \mathrm{~S} / 6 \mathrm{E}-21 \mathrm{H} 3$ & $210-550$ & 200 & $33^{\circ} 43^{\prime} 17^{\prime \prime} \mathrm{N}$ & $116^{\circ} 21^{\prime} 33^{\prime \prime} \mathrm{W}$ & LP & 216 \\
\hline $5 \mathrm{~S} / 6 \mathrm{E}-21 \mathrm{H} 5$ & $400-600$ & 200 & $33^{\circ} 43^{\prime} 18^{\prime \prime} \mathrm{N}$ & $116^{\circ} 21^{\prime} 27^{\prime \prime} \mathrm{W}$ & LP & 216 \\
\hline $5 S / 6 E-21 J 1$ & $172-278$ & 200 & $33^{\circ} 43^{\prime} 14^{\prime \prime} \mathrm{N}$ & $116^{\circ} 21^{\prime} 33^{\prime \prime} \mathrm{W}$ & LP & 216 \\
\hline
\end{tabular}


Appendix 1: List and description of wells in data base--Continued

\begin{tabular}{|c|c|c|c|c|c|c|}
\hline $\begin{array}{l}\text { Well } \\
\text { number }\end{array}$ & $\begin{array}{c}\text { Perforated } \\
\text { interval (depth, in } \\
\text { feet below land } \\
\text { surface) }\end{array}$ & $\begin{array}{l}\text { Land-surface } \\
\text { altitude (feet } \\
\text { above or } \\
\text { below (-) sea } \\
\text { level) }\end{array}$ & Latitude & Longitude & Remarks & $\begin{array}{c}\text { Closest } \\
\text { model node } \\
\text { for pumped } \\
\text { wells (in } \\
\text { model area } \\
\text { only) }\end{array}$ \\
\hline $5 S / 6 E-21 L 1$ & $250-578$ & 240 & $33^{\circ} 43^{\prime} 08^{\prime \prime} \mathrm{N}$ & $116^{\circ} 22^{\prime} 05^{\prime \prime} \mathrm{W}$ & L & \\
\hline $5 S / 6 E-21 N 1$ & $200-243$ & 248 & $33^{\circ} 43^{\prime} 03^{\prime \prime} \mathrm{N}$ & $116^{\circ} 22^{\prime} 11^{\prime \prime} \mathrm{W}$ & $\mathrm{L}$ & \\
\hline $5 S / 6 E-21 N 2$ & $400-820$ & 248 & $33^{\circ} 43^{\prime} 04^{\prime \prime} \mathrm{N}$ & $116^{\circ} 22^{\prime} 10^{\prime \prime} \mathrm{W}$ & LP & 215 \\
\hline $5 S / 6 E-21 P 1$ & $212-433$ & 260 & $33^{\circ} 42^{\prime} 52^{\prime \prime} \mathrm{N}$ & $116^{\circ} 21^{\prime} 57^{\prime \prime} \mathrm{W}$ & LP & 215 \\
\hline $5 \mathrm{~S} / 6 \mathrm{E}-21 \mathrm{Q} 3$ & $350-700$ & 240 & $33^{\circ} 42^{\prime} 59^{\prime \prime} \mathrm{N}$ & $116^{\circ} 21^{\prime} 43^{\prime \prime} \mathrm{W}$ & LP & 215 \\
\hline $5 \mathrm{~S} / 6 \mathrm{E}-21 \mathrm{R} 1$ & $250-480$ & 217 & $33^{\circ} 42^{\prime} 59^{\prime \prime} \mathrm{N}$ & $116^{\circ} 21^{\prime} 30^{\prime \prime} \mathrm{W}$ & LP & 226 \\
\hline $5 \mathrm{~S} / 6 \mathrm{E}-22 \mathrm{~B} 1$ & $\begin{array}{l}350-390 \\
416-452\end{array}$ & 160 & $33^{\circ} 43^{\prime} 33^{\prime \prime} \mathrm{N}$ & $116^{\circ} 20^{\prime} 47^{\prime \prime} \mathrm{W}$ & LP & 227 \\
\hline $5 \mathrm{~S} / 6 \mathrm{E}-22 \mathrm{~B} 2$ & $\begin{array}{l}468-502 \\
548-704 \\
752-872\end{array}$ & 150 & $33^{\circ} 43^{\prime} 42^{\prime \prime} \mathrm{N}$ & $116^{\circ} 20^{\prime} 43^{\prime \prime} \mathrm{W}$ & LP & 228 \\
\hline $5 \mathrm{~S} / 6 \mathrm{E}-22 \mathrm{C} 1$ & $183-510$ & 160 & $33^{\circ} 43^{\prime} 38^{\prime \prime} \mathrm{N}$ & $116^{\circ} 20^{\prime} 54^{\prime \prime} \mathrm{W}$ & & \\
\hline $5 \mathrm{~S} / 6 \mathrm{E}-22 \mathrm{G} 2$ & $288-474$ & 165 & $33^{\circ} 43^{\prime} 24^{\prime \prime} \mathrm{N}$ & $116^{\circ} 20^{\prime} 47^{\prime \prime} \mathrm{W}$ & LP & 227 \\
\hline $5 \mathrm{~S} / 6 \mathrm{E}-22 \mathrm{H} 1$ & $291-507$ & 150 & $33^{\circ} 43^{\prime} 26^{\prime \prime} \mathrm{N}$ & $116^{\circ} 20^{\prime} 25^{\prime \prime} \mathrm{W}$ & LP & 227 \\
\hline $5 \mathrm{~S} / 6 \mathrm{E}-22 \mathrm{H} 2$ & $772-855$ & 150 & $33^{\circ} 43^{\prime} 25^{\prime \prime} \mathrm{N}$ & $116^{\circ} 20^{\prime} 20^{\prime \prime} \mathrm{W}$ & LP & 227 \\
\hline $5 \mathrm{~S} / 6 \mathrm{E}-22 \mathrm{~J} 1$ & $\begin{array}{l}342-372 \\
388-392 \\
416-518 \\
524-554\end{array}$ & 175 & $33^{\circ} 43^{\prime} 05^{\prime \prime} \mathrm{N}$ & $116^{\circ} 20^{\prime} 31^{\prime \prime} \mathrm{W}$ & LP & 227 \\
\hline $5 \mathrm{~S} / 6 \mathrm{E}-22 \mathrm{M} 1$ & $\begin{array}{l}222-260 \\
287-340\end{array}$ & 200 & $33^{\circ} 43^{\prime} 06^{\prime \prime} \mathrm{N}$ & $116^{\circ} 21^{\prime} 13^{\prime \prime} \mathrm{W}$ & LP & 227 \\
\hline $5 \mathrm{~S} / 6 \mathrm{E}-22 \mathrm{P} 1$ & $\begin{array}{l}254-292 \\
305-370\end{array}$ & 198 & $33^{\circ} 42^{\prime} 59^{\prime \prime} \mathrm{N}$ & $116^{\circ} 21^{\prime} 02^{\prime \prime} \mathrm{W}$ & LP & 227 \\
\hline $5 \mathrm{~S} / 6 \mathrm{E}-22 \mathrm{P} 2$ & $264-370$ & 205 & $33^{\circ} 42^{\prime} 52^{\prime \prime} \mathrm{N}$ & $116^{\circ} 20^{\prime} 56^{\prime \prime} \mathrm{W}$ & LP & 227 \\
\hline $5 \mathrm{~S} / 6 \mathrm{E}-23 \mathrm{E} 2$ & $\begin{array}{l}374-383 \\
400-413 \\
482-509\end{array}$ & 144 & $33^{\circ} 43^{\prime} 21^{\prime \prime} \mathrm{N}$ & $116^{\circ} 20^{\prime} 15^{\prime \prime} \mathrm{W}$ & LP & 227 \\
\hline $5 \mathrm{~S} / 6 \mathrm{E}-23 \mathrm{E} 3$ & $275-420$ & 140 & $33^{\circ} 43^{\prime} 22^{\prime \prime} \mathrm{N}$ & $116^{\circ} 20^{\prime} 10^{\prime \prime} \mathrm{W}$ & LP & 227 \\
\hline $5 \mathrm{~S} / 6 \mathrm{E}-23 \mathrm{~F} 1$ & $778-794$ & 140 & $33^{\circ} 43^{\prime} 23^{\prime \prime} \mathrm{N}$ & $116^{\circ} 19^{\prime} 51^{\prime \prime} \mathrm{W}$ & LP & 228 \\
\hline $5 \mathrm{~S} / 6 \mathrm{E}-23 \mathrm{H} 1$ & & 125 & $33^{\circ} 43^{\prime} 22^{\prime \prime} \mathrm{N}$ & $116^{\circ} 19^{\prime} 29^{\prime \prime} \mathrm{W}$ & $\mathrm{L}$ & 236 \\
\hline $5 \mathrm{~S} / 6 \mathrm{E}-23 \mathrm{~K} 2$ & $476-492$ & 135 & $33^{\circ} 43^{\prime} 13^{\prime \prime} \mathrm{N}$ & $116^{\circ} 19^{\prime} 40^{\prime \prime} \mathrm{W}$ & LP & 235 \\
\hline $5 \mathrm{~S} / 6 \mathrm{E}-23 \mathrm{~L} 1$ & $\begin{array}{l}110-168 \\
280-352 \\
355-426\end{array}$ & 129 & $33^{\circ} 43^{\prime} 15^{\prime \prime} \mathrm{N}$ & $116^{\circ} 19^{\prime} 59^{\prime \prime} \mathrm{W}$ & LP & 227 \\
\hline $5 S / 6 E-23 L 3$ & $255-312$ & 130 & $33^{\circ} 43^{\prime} 12^{\prime \prime} \mathrm{N}$ & $116^{\circ} 19^{\prime} 57^{\prime \prime} \mathrm{W}$ & $\mathrm{L}$ & \\
\hline $5 \mathrm{~S} / 6 \mathrm{E}-23 \mathrm{M} 1$ & $\begin{array}{l}384-480 \\
576-776\end{array}$ & 160 & $33^{\circ} 43^{\prime} 07^{\prime \prime} \mathrm{N}$ & $116^{\circ} 20^{\prime} 15^{\prime \prime} \mathrm{W}$ & LP & 227 \\
\hline $5 \mathrm{~S} / 6 \mathrm{E}-23 \mathrm{~N} 1$ & $504-577$ & 160 & $33^{\circ} 43^{\prime} 01^{\prime \prime} \mathrm{N}$ & $116^{\circ} 20^{\prime} 03^{\prime \prime} \mathrm{W}$ & LP & 227 \\
\hline $5 \mathrm{~S} / 6 \mathrm{E}-24 \mathrm{D} 1$ & -. & 160 & $33^{\circ} 43^{\prime} 41^{\prime \prime} \mathrm{N}$ & $116^{\circ} 19^{\prime} 09^{\prime \prime} \mathrm{W}$ & LP & 236 \\
\hline $5 S / 6 E-24 F 1$ & $440-690$ & 155 & $33^{\circ} 43^{\prime} 29^{\prime \prime} \mathrm{N}$ & $116^{\circ} 18^{\prime} 55^{\prime \prime} \mathrm{W}$ & LP & 236 \\
\hline $5 \mathrm{~S} / 6 \mathrm{E}-24 \mathrm{G} 1$ & $\begin{array}{l}480-680 \\
810-930\end{array}$ & 110 & $33^{\circ} 43^{\prime} 18^{\prime \prime} \mathrm{N}$ & $116^{\circ} 18^{\prime} 32^{\prime \prime} \mathrm{W}$ & LP & 243 \\
\hline $5 \mathrm{~S} / 6 \mathrm{E}-24 \mathrm{M} 1$ & $\begin{array}{l}396-564 \\
604-628 \\
668-778\end{array}$ & 115 & $33^{\circ} 43^{\prime} 13^{\prime \prime} \mathrm{N}$ & $116^{\circ} 18^{\prime} 59^{\prime \prime} W$ & LP & 236 \\
\hline $5 \mathrm{~S} / 6 \mathrm{E}-24 \mathrm{R} 1$ & -- & 94 & $33^{\circ} 43^{\prime} 02^{\prime \prime} \mathrm{N}$ & $116^{\circ} 18^{\prime} 25^{\prime \prime} \mathrm{W}$ & $\mathrm{L}$ & \\
\hline $5 \mathrm{~S} / 6 \mathrm{E}-25 \mathrm{~A} 1$ & $400-656$ & 91 & $33^{\circ} 42^{\prime} 45^{\prime \prime} \mathrm{N}$ & $116^{\circ} 18^{\circ} 11^{\prime \prime} \mathrm{W}$ & LP & 242 \\
\hline $5 \mathrm{~S} / 6 \mathrm{E}-25 \mathrm{~A} 2$ & $430-650$ & 80 & $33^{\circ} 42^{\prime} 47^{\prime \prime} \mathrm{N}$ & $116^{\circ} 18^{\prime} 17^{\prime \prime} \mathrm{W}$ & LP & 242 \\
\hline $5 \mathrm{~S} / 6 \mathrm{E}-27 \mathrm{~A} 1$ & $458-596$ & 160 & $33^{\circ} 42^{\prime} 45^{\prime \prime} \mathrm{N}$ & $116^{\circ} 20^{\prime} 17^{\prime \prime} \mathrm{W}$ & LP & 227 \\
\hline $5 S / 6 E-27 A 2$ & $322-482$ & 170 & $33^{\circ} 42^{\prime} 48^{\prime \prime} \mathrm{N}$ & $116^{\circ} 20^{\prime} 25^{\prime \prime} \mathrm{W}$ & LP & 227 \\
\hline $5 \mathrm{~S} / 6 \mathrm{E}-27 \mathrm{~B} 2$ & $178-298$ & 200 & $33^{\circ} 42^{\prime} 50^{\prime \prime} \mathrm{N}$ & $116^{\circ} 20^{\prime} 37^{\prime \prime} \mathrm{W}$ & LP & 227 \\
\hline $5 S / 6 E-27 C 1$ & $138-292$ & 204 & $33^{\circ} 42^{\prime} 49^{\prime \prime} \mathrm{N}$ & $116^{\circ} 20^{\prime} 50^{\prime \prime} \mathrm{W}$ & LP & 227 \\
\hline $5 \mathrm{~S} / 6 \mathrm{E}-27 \mathrm{C} 2$ & -. & 211 & $33^{\circ} 42^{\prime} 49^{\prime \prime} \mathrm{N}$ & $116^{\circ} 21^{\prime} 02^{\prime \prime} \mathrm{W}$ & LP & 226 \\
\hline $5 S / 6 \mathrm{E}-27 \mathrm{D} 1$ & $266-392$ & 225 & $33^{\circ} 42^{\prime} 44^{\prime \prime} \mathrm{N}$ & $116^{\circ} 21^{\prime} 12^{\prime \prime} \mathrm{W}$ & LP & 226 \\
\hline
\end{tabular}


Appendix 1: List and description of wells in data base--Continued

\begin{tabular}{|c|c|c|c|c|c|c|}
\hline $\begin{array}{c}\text { Well } \\
\text { number }\end{array}$ & $\begin{array}{c}\text { Perforated } \\
\text { interval (depth, in } \\
\text { feet below land } \\
\text { surface) }\end{array}$ & $\begin{array}{c}\text { Land-surface } \\
\text { altitude (feet } \\
\text { above or } \\
\text { below (-) sea } \\
\text { level) }\end{array}$ & Latitude & Longitude & Remarks & $\begin{array}{c}\text { Closest } \\
\text { model node } \\
\text { for pumped } \\
\text { wells (in } \\
\text { model area } \\
\text { only) }\end{array}$ \\
\hline $5 S / 6 E-27 D 2$ & $170-370$ & 230 & $33^{\circ} 42^{\prime} 39^{\prime \prime} \mathrm{N}$ & $116^{\circ} 21^{\prime} 15^{\prime \prime} \mathrm{W}$ & LP & 226 \\
\hline $5 \mathrm{~S} / 6 \mathrm{E}-28 \mathrm{C} 1$ & $204-252$ & 262 & $33^{\circ} 42^{\prime} 50^{\prime \prime} \mathrm{N}$ & $116^{\circ} 21^{\prime} 52^{\prime \prime} \mathrm{W}$ & $\mathrm{L}$ & \\
\hline $5 \mathrm{~S} / 6 \mathrm{E}-28 \mathrm{C} 2$ & $410-680$ & 262 & $33^{\circ} 42^{\prime} 49^{\prime \prime} \mathrm{N}$ & $116^{\circ} 21^{\prime} 52^{\prime \prime} \mathrm{W}$ & LP & 215 \\
\hline $5 \mathrm{~S} / 6 \mathrm{E}-28 \mathrm{E} 1$ & $247-346$ & 332 & $33^{\circ} 42^{\prime} 25^{\prime \prime} \mathrm{N}$ & $116^{\circ} 22^{\prime} 19^{\prime \prime} \mathrm{W}$ & $\mathrm{L}$ & \\
\hline $5 \mathrm{~S} / 6 \mathrm{E}-28 \mathrm{E} 2$ & $320-470$ & 310 & $33^{\circ} 42^{\prime} 35^{\prime \prime} \mathrm{N}$ & $116^{\circ} 22^{\prime} 19^{\prime \prime} \mathrm{W}$ & LP & 215 \\
\hline $5 \mathrm{~S} / 6 \mathrm{E}-28 \mathrm{H} 1$ & $194-373$ & 260 & $33^{\circ} 42^{\prime} 29^{\prime \prime} \mathrm{N}$ & $116^{\circ} 21^{\prime} 29^{\prime \prime} \mathrm{W}$ & LP & 226 \\
\hline $5 \mathrm{~S} / 6 \mathrm{E}-29 \mathrm{~B} 1$ & $351-615$ & 290 & $33^{\circ} 42^{\prime} 48^{\prime \prime} \mathrm{N}$ & $116^{\circ} 22^{\prime} 43^{\prime \prime} \mathrm{W}$ & LP & 215 \\
\hline $5 S / 6 E-29 C 1$ & $243-531$ & 337 & $33^{\circ} 42^{\prime} 41^{\prime \prime} \mathrm{N}$ & $116^{\circ} 23^{\prime} 01^{\prime \prime} \mathrm{W}$ & $\mathrm{L}$ & \\
\hline $5 S / 6 E-29 C 2$ & $530-670$ & 337 & $33^{\circ} 42^{\prime} 40^{\prime \prime} \mathrm{N}$ & $116^{\circ} 23^{\prime} 01^{\prime \prime} \mathrm{W}$ & LP & 214 \\
\hline $5 \mathrm{~S} / 6 \mathrm{E}-29 \mathrm{H} 1$ & -. & 320 & $33^{\circ} 42^{\prime} 34^{\prime \prime} \mathrm{N}$ & $116^{\circ} 22^{\prime} 42^{\prime \prime} \mathrm{W}$ & LP & 214 \\
\hline $5 \mathrm{~S} / 6 \mathrm{E}-29 \mathrm{M} 1$ & $457-723$ & 405 & $33^{\circ} 42^{\prime} 23^{\prime \prime} \mathrm{N}$ & $116^{\circ} 23^{\prime} 10^{\prime \prime} \mathrm{W}$ & LP & 214 \\
\hline $5 \mathrm{~S} / 6 \mathrm{E}-29 \mathrm{P} 1$ & $500-700$ & 454 & $33^{\circ} 42^{\prime} 07^{\prime \prime} \mathrm{N}$ & $116^{\circ} 23^{\prime} 08^{\prime \prime} \mathrm{W}$ & LP & 214 \\
\hline $5 \mathrm{~S} / 6 \mathrm{E}-32 \mathrm{~B} 1$ & $450-700$ & 440 & $33^{\circ} 41^{\prime} 53^{\prime \prime} \mathrm{N}$ & $116^{\circ} 22^{\prime} 44^{\prime \prime} \mathrm{W}$ & LP & 213 \\
\hline $5 \mathrm{~S} / 6 \mathrm{E}-32 \mathrm{~B} 2$ & $460-700$ & 440 & $33^{\circ} 41^{\prime} 53^{\prime \prime} \mathrm{N}$ & $116^{\circ} 22^{\prime} 44^{\prime \prime} \mathrm{W}$ & LP & 213 \\
\hline $5 \mathrm{~S} / 6 \mathrm{E}-32 \mathrm{~B} 3$ & $460-652$ & 440 & $33^{\circ} 41^{\prime} 53^{\prime \prime} \mathrm{N}$ & $116^{\circ} 22^{\prime} 44^{\prime \prime} \mathrm{W}$ & LP & 213 \\
\hline $5 \mathrm{~S} / 6 \mathrm{E}-32 \mathrm{G} 1$ & $459-736$ & 455 & $33^{\circ} 41^{\prime} 33^{\prime \prime} \mathrm{N}$ & $116^{\circ} 22^{\prime} 38^{\prime \prime} \mathrm{W}$ & $\mathrm{L}$ & \\
\hline $5 \mathrm{~S} / 7 \mathrm{E}-03 \mathrm{~K} 1$ & $580-740$ & 44 & $33^{\circ} 45^{\prime} 50^{\prime \prime} \mathrm{N}$ & $116^{\circ} 14^{\prime} 24^{\prime \prime} \mathrm{W}$ & $\overline{\mathrm{L}}$ & \\
\hline $5 \mathrm{~S} / 7 \mathrm{E}-04 \mathrm{~A} 1$ & $147-367$ & 47 & $33^{\circ} 46^{\prime} 22^{\prime \prime} \mathrm{N}$ & $116^{\circ} 15^{\prime} 14^{\prime \prime} \mathrm{W}$ & L & \\
\hline $5 S / 7 E-04 C 1$ & $520-800$ & 50 & $33^{\circ} 46^{\prime} 19^{\prime \prime} \mathrm{N}$ & $116^{\circ} 15^{\prime} 42^{\prime \prime} \mathrm{W}$ & $\mathrm{L}$ & \\
\hline 5S/7E-04D1 & $100-370$ & 58 & $33^{\circ} 46^{\prime} 19^{\prime \prime} \mathrm{N}$ & $116^{\circ} 15^{\prime} 56^{\prime \prime} \mathrm{W}$ & L & \\
\hline $5 \mathrm{~S} / 7 \mathrm{E}-04 \mathrm{H} 1$ & $570-840$ & 42 & $33^{\circ} 46^{\prime} 08^{\prime \prime} \mathrm{N}$ & $116^{\circ} 15^{\prime} 07^{\prime \prime} \mathrm{W}$ & $\mathbf{L}$ & \\
\hline $5 \mathrm{~S} / 7 \mathrm{E}-04 \mathrm{M} 1$ & $192-678$ & 51 & $33^{\circ} 45^{\prime} 55^{\prime \prime} \mathrm{N}$ & $116^{\circ} 16^{\prime} 01^{\prime \prime} \mathrm{W}$ & $\bar{L}$ & \\
\hline $5 \mathrm{~S} / 7 \mathrm{E}-04 \mathrm{~N} 1$ & $147-411$ & 51 & $33^{\circ} 45^{\prime} 36^{\prime \prime} \mathrm{N}$ & $116^{\circ} 15^{\prime} 20^{\prime \prime} \mathrm{W}$ & $\overline{\mathrm{L}}$ & \\
\hline $5 S / 7 E-04 Q 1$ & $123-363$ & 38 & $33^{\circ} 45^{\prime} 36^{\prime \prime} \mathrm{N}$ & $116^{\circ} 15^{\prime} 20^{\prime \prime} \mathrm{W}$ & L & \\
\hline $5 S / 7 E-04 Q 3$ & $240-320$ & 40 & $33^{\circ} 45^{\prime} 39^{\prime \prime} \mathrm{N}$ & $116^{\circ} 15^{\prime} 31^{\prime \prime} \mathrm{W}$ & $\mathrm{L}$ & \\
\hline $5 S / 7 E-05 C 1$ & $260-400$ & 69 & $33^{\circ} 46^{\prime} 19^{\prime \prime} \mathrm{N}$ & $116^{\circ} 16^{\prime} 45^{\prime \prime} \mathrm{W}$ & $\mathrm{L}$ & \\
\hline $5 \mathrm{~S} / 7 \mathrm{E}-05 \mathrm{~K} 1$ & -- & 61 & $33^{\circ} 45^{\prime} 43^{\prime \prime} \mathrm{N}$ & $116^{\circ} 16^{\prime} 28^{\prime \prime} \mathrm{W}$ & LP & 246 \\
\hline $5 \mathrm{~S} / 7 \mathrm{E}-05 \mathrm{R} 1$ & $230-350$ & 59 & $33^{\circ} 45^{\prime} 33^{\prime \prime} \mathrm{N}$ & $116^{\circ} 16^{\prime} 15^{\prime \prime} \mathrm{W}$ & $\mathrm{L}$ & \\
\hline 5S/7E-06B1 & $\begin{array}{l}168-180 \\
280-303\end{array}$ & 92 & $33^{\circ} 46^{\prime} 21^{\prime \prime} \mathrm{N}$ & $116^{\circ} 17^{\prime} 25^{\prime \prime} \mathrm{W}$ & LP & 240 \\
\hline 5S/7E-06B3 & $300-660$ & 90 & $33^{\circ} 46^{\prime} 19^{\prime \prime} \mathrm{N}$ & $116^{\circ} 17^{\prime} 30^{\prime \prime} \mathrm{W}$ & LP & 239 \\
\hline $5 \mathrm{~S}^{\prime} / 7 \mathrm{E}-06 \mathrm{H} 1$ & $400-480$ & 82 & $33^{\circ} 46^{\prime} 06^{\prime \prime} \mathrm{N}$ & $116^{\circ} 17^{\prime} 12^{\prime \prime} \mathrm{W}$ & $\mathbf{L}$ & \\
\hline 5S/7E-06M1 & $\begin{array}{l}195-363 \\
555-651\end{array}$ & 103 & $33^{\circ} 45^{\prime} 48^{\prime \prime} \mathrm{N}$ & $116^{\circ} 18^{\prime} 00^{\prime \prime} \mathrm{W}$ & LP & 239 \\
\hline 5S/7E-07F1 & $147-459$ & 102 & $33^{\circ} 45^{\prime} 15^{\prime \prime} \mathrm{N}$ & $116^{\circ} 17^{\prime} 51^{\prime \prime} \mathrm{W}$ & LP & 245 \\
\hline $5 \mathrm{~S} / 7 \mathrm{E}-07 \mathrm{~J} 1$ & $147-363$ & 100 & $33^{\circ} 45^{\prime} 01^{\prime \prime} \mathrm{N}$ & $116^{\circ} 17^{\prime} 15^{\prime \prime} \mathrm{W}$ & LP & 246 \\
\hline $5 \mathrm{~S} / 7 \mathrm{E}-07 \mathrm{P} 1$ & $144-450$ & 101 & $33^{\circ} 44^{\prime} 42^{\prime \prime} \mathrm{N}$ & $116^{\circ} 17^{\prime} 44^{\prime \prime} \mathrm{W}$ & LP & 245 \\
\hline $5 \mathrm{~S} / 7 \mathrm{E}-08 \mathrm{~A} 2$ & $286-650$ & 55 & $33^{\circ} 45^{\prime} 18^{\prime \prime} \mathrm{N}$ & $116^{\circ} 16^{\prime} 07^{\prime \prime} \mathrm{W}$ & $\mathrm{L}$ & \\
\hline $5 \mathrm{~S} / 7 \mathrm{E}-08 \mathrm{G} 1$ & -- & 85 & $33^{\circ} 45^{\prime} 06^{\prime \prime} \mathrm{N}$ & $116^{\circ} 16^{\prime} 24^{\prime \prime} \mathrm{W}$ & $\mathbf{L}$ & \\
\hline $5 \mathrm{~S} / 7 \mathrm{E}-08 \mathrm{Q} 1$ & $203-654$ & 54 & $33^{\circ} 44^{\prime} 49^{\prime \prime} \mathrm{N}$ & $116^{\circ} 16^{\prime} 20^{\prime \prime} \mathrm{W}$ & $\mathrm{L}$ & \\
\hline $5 S / 7 E-09 F 1$ & $130-310$ & 39 & $33^{\circ} 45^{\prime} 11^{\prime \prime} \mathrm{N}$ & $116^{\circ} 15^{\prime} 34^{\prime \prime} \mathrm{W}$ & $\mathrm{L}$ & \\
\hline $5 \mathrm{~S} / 7 \mathrm{E}-09 \mathrm{~K} 1$ & $147-387$ & 33 & $33^{\circ} 44^{\prime} 55^{\prime \prime} \mathrm{N}$ & $116^{\circ} 15^{\prime} 27^{\prime \prime} \mathrm{W}$ & $\mathrm{L}$ & \\
\hline $5 \mathrm{~S} / 7 \mathrm{E}-09 \mathrm{~L} 2$ & $147-319$ & 41 & $33^{\circ} 44^{\prime} 56^{\prime \prime} \mathrm{N}$ & $116^{\circ} 15^{\prime} 42^{\prime \prime} \mathrm{W}$ & $\mathrm{L}$ & \\
\hline $5 \mathrm{~S} / 7 \mathrm{E}-10 \mathrm{D} 2$ & $200-530$ & 32 & $33^{\circ} 45^{\prime} 23^{\prime \prime} \mathrm{N}$ & $116^{\circ} 14^{\prime} 54^{\prime \prime} \mathrm{W}$ & $\mathrm{L}$ & \\
\hline $5 \mathrm{~S} / 7 \mathrm{E}-10 \mathrm{E} 1$ & $70-360$ & 28 & $33^{\circ} 45^{\prime} 17^{\prime \prime} \mathrm{N}$ & $116^{\circ} 14^{\prime} 58^{\prime \prime} \mathrm{W}$ & $\mathbf{L}$ & \\
\hline $5 S / 7 E-11 C 1$ & $323-443$ & 29 & $33^{\circ} 45^{\prime} 22^{\prime \prime} \mathrm{N}$ & $116^{\circ} 13^{\prime} 35^{\prime \prime} \mathrm{W}$ & $\mathrm{L}$ & \\
\hline $5 \mathrm{~S} / 7 \mathrm{E}-12 \mathrm{P} 1$ & $280-400$ & 3 & $33^{\circ} 44^{\prime} 44^{\prime \prime} \mathrm{N}$ & $116^{\circ} 12^{\prime} 34^{\prime \prime} \mathrm{W}$ & $\mathbf{L}$ & \\
\hline 5S/7E-13D1 & $\begin{array}{c}580-583 \\
980-1,022\end{array}$ & -11 & $33^{\circ} 44^{\prime} 32^{\prime \prime} \mathrm{N}$ & $116^{\circ} 12^{\prime} 51^{\prime \prime} \mathrm{W}$ & $\mathrm{L}$ & \\
\hline $5 \mathrm{~S} / 7 \mathrm{E}-14 \mathrm{~J} 2$ & $232-350$ & -12 & $33^{\circ} 44^{\prime} 05^{\prime \prime} \mathrm{N}$ & $116^{\circ} 13^{\prime} 04^{\prime \prime} \mathrm{W}$ & $\mathrm{L}$ & \\
\hline $5 S / 7 E-14 K 1$ & $264-411$ & -5 & $33^{\circ} 44^{\prime} 05^{\prime \prime} \mathrm{N}$ & $116^{\circ} 13^{\prime} 23^{\prime \prime} \mathrm{W}$ & $\mathrm{L}$ & \\
\hline $5 \mathrm{~S} / 7 \mathrm{E}-15 \mathrm{Q} 1$ & $264-464$ & 5 & $33^{\circ} 43^{\prime} 52^{\prime \prime} \mathrm{N}$ & $116^{\circ} 14^{\prime} 19^{\prime \prime} \mathrm{W}$ & $\mathbf{L}$ & \\
\hline $5 \mathrm{~S} / 7 \mathrm{E}-16 \mathrm{C} 1$ & $147-355$ & 37 & $33^{\circ} 44^{\prime} 25^{\prime \prime} \mathrm{N}$ & $116^{\circ} 15^{\prime} 41^{\prime \prime} \mathrm{W}$ & $\mathbf{L}$ & \\
\hline $5 S / 7 E-16 K 2$ & $200-415$ & 27 & $33^{\circ} 44^{\prime} 10^{\prime \prime} \mathrm{N}$ & $116^{\circ} 15^{\prime} 26^{\prime \prime} \mathrm{W}$ & LP & \\
\hline
\end{tabular}


Appendix 1: List and description of wells in data base--Continued

\begin{tabular}{|c|c|c|c|c|c|c|}
\hline $\begin{array}{c}\text { Well } \\
\text { number }\end{array}$ & $\begin{array}{c}\text { Perforated } \\
\text { interval (depth, in } \\
\text { feet below land } \\
\text { surface) }\end{array}$ & $\begin{array}{c}\text { Land-surface } \\
\text { altitude (feet } \\
\text { above or } \\
\text { below (-) sea } \\
\text { level) }\end{array}$ & Latitude & Longitude & Remarks & $\begin{array}{c}\text { Closest } \\
\text { model node } \\
\text { for pumped } \\
\text { wells (in } \\
\text { model area } \\
\text { only) }\end{array}$ \\
\hline 5S/7E-17E1 & $459-603$ & 82 & $33^{\circ} 44^{\prime} 13^{\prime \prime} \mathrm{N}$ & $116^{\circ} 16^{\prime} 52^{\prime \prime} \mathrm{W}$ & L & \\
\hline $5 \mathrm{~S} / 7 \mathrm{E}-17 \mathrm{~F} 1$ & $200-720$ & 80 & $33^{\circ} 44^{\prime} 18^{\prime \prime} \mathrm{N}$ & $116^{\circ} 16^{\prime} 46^{\prime \prime} \mathrm{W}$ & $\mathbf{L}$ & \\
\hline $5 \mathrm{~S} / 7 \mathrm{E}-17 \mathrm{~L} 1$ & $212-600$ & 67 & $33^{\circ} 44^{\prime} 03^{\prime \prime} \mathrm{N}$ & $116^{\circ} 16^{\prime} 45^{\prime \prime} \mathrm{W}$ & $\overline{\mathbf{L}}$ & \\
\hline $5 S / 7 E-18 D 1$ & $160-200$ & 129 & $33^{\circ} 44^{\prime} 25^{\prime \prime} \mathrm{N}$ & $116^{\circ} 18^{\prime} 04^{\prime \prime} \mathrm{W}$ & $\bar{L}$ & \\
\hline $5 \mathrm{~S} / 7 \mathrm{E}-18 \mathrm{~F} 1$ & -- & 112 & $33^{\circ} 44^{\prime} 15^{\prime \prime} \mathrm{N}$ & $116^{\circ} 17^{\prime} 48^{\prime \prime} \mathrm{W}$ & LP & 244 \\
\hline $5 S / 7 E-18 M 2$ & $168-264$ & 127 & $33^{\circ} 44^{\prime} 01^{\prime \prime} \mathrm{N}$ & $116^{\circ} 17^{\prime} 55^{\prime \prime} \mathrm{W}$ & $\mathbf{L}$ & \\
\hline 5S/7E-19D1 & $\begin{array}{l}440-650 \\
710-770 \\
840-920\end{array}$ & 140 & $33^{\circ} 43^{\prime} 37^{\prime \prime} \mathrm{N}$ & $116^{\circ} 18^{\prime} 33^{\prime \prime} \mathrm{W}$ & LP & 244 \\
\hline $5 \mathrm{~S} / 7 \mathrm{E}-19 \mathrm{H} 2$ & $460-660$ & 104 & $33^{\circ} 43^{\prime} 22^{\prime \prime} \mathrm{N}$ & $116^{\circ} 17^{\prime} 11^{\prime \prime} \mathrm{W}$ & L & \\
\hline $5 \mathrm{~S} / 7 \mathrm{E}-20 \mathrm{P} 2$ & $150-350$ & 75 & $33^{\circ} 42^{\prime} 58^{\prime \prime} \mathrm{N}$ & $116^{\circ} 16^{\prime} 44^{\prime \prime} \mathrm{W}$ & $\mathbf{L}$ & \\
\hline $5 \mathrm{~S} / 7 \mathrm{E}-21 \mathrm{~F} 2$ & $82-614$ & 28 & $33^{\circ} 43^{\prime} 22^{\prime \prime} \mathrm{N}$ & $116^{\circ} 15^{\prime} 34^{\prime \prime} \mathrm{W}$ & $\bar{L}$ & \\
\hline $5 \mathrm{~S} / 7 \mathrm{E}-21 \mathrm{Q} 1$ & -- & 40 & $33^{\circ} 42^{\prime} 55^{\prime \prime} \mathrm{N}$ & $116^{\circ} 15^{\prime} 30^{\prime \prime} \mathrm{W}$ & $\mathbf{L}$ & \\
\hline $5 \mathrm{~S} / 7 \mathrm{E}-22 \mathrm{H} 2$ & $506-1,100$ & 5 & $33^{\circ} 43^{\prime} 31^{\prime \prime} \mathrm{N}$ & $116^{\circ} 14^{\prime} 06^{\prime \prime} \mathrm{W}$ & L & \\
\hline $5 \mathrm{~S} / 7 \mathrm{E}-23 \mathrm{D} 2$ & $483-882$ & 0 & $33^{\circ} 43^{\prime} 39^{\prime \prime} \mathrm{N}$ & $116^{\circ} 13^{\prime} 54^{\prime \prime} \mathrm{W}$ & $\mathrm{L}$ & \\
\hline $5 \mathrm{~S} / 7 \mathrm{E}-24 \mathrm{M} 4$ & $250-660$ & -17 & $33^{\circ} 43^{\prime} 14^{\prime \prime} \mathrm{N}$ & $116^{\circ} 12^{\prime} 44^{\prime \prime} \mathrm{W}$ & L & \\
\hline $5 \mathrm{~S} / 7 \mathrm{E}-25 \mathrm{R} 1$ & -. & -30 & $33^{\circ} 42^{\prime} 07^{\prime \prime} \mathrm{N}$ & $116^{\circ} 12^{\prime} 14^{\prime \prime} \mathrm{W}$ & $\bar{L}$ & \\
\hline $5 S / 7 E-26 E 3$ & $515-1,110$ & 5 & $33^{\circ} 42^{\prime} 27^{\prime \prime} \mathrm{N}$ & $116^{\circ} 13^{\prime} 50^{\prime \prime} \mathrm{W}$ & L & \\
\hline $5 \mathrm{~S} / 7 \mathrm{E}-27 \mathrm{~B} 1$ & $236-500$ & 16 & $33^{\circ} 42^{\prime} 40^{\prime \prime} \mathrm{N}$ & $116^{\circ} 14^{\prime} 28^{\prime \prime} \mathrm{W}$ & $\mathrm{L}$ & \\
\hline $5 \mathrm{~S} / 7 \mathrm{E}-27 \mathrm{~L} 1$ & $516-600$ & 20 & $33^{\circ} 42^{\prime} 22^{\prime \prime} \mathrm{N}$ & $116^{\circ} 14^{\prime} 38^{\prime \prime} \mathrm{W}$ & $\mathrm{L}$ & \\
\hline $5 S / 7 E-28 E 1$ & $128-198$ & 46 & $33^{\circ} 42^{\prime} 35^{\prime \prime} \mathrm{N}$ & $116^{\circ} 15^{\prime} 56^{\prime \prime} \mathrm{W}$ & LP & \\
\hline $5 S / 7 E-29 K 1$ & -. & 60 & $33^{\circ} 42^{\prime} 19^{\prime \prime} \mathrm{N}$ & $116^{\circ} 16^{\prime} 28^{\prime \prime} \mathrm{W}$ & L & \\
\hline $5 \mathrm{~S} / 7 \mathrm{E}-30 \mathrm{C} 2$ & $\begin{array}{l}115-235 \\
427-489\end{array}$ & 73 & $33^{\circ} 42^{\prime} 42^{\prime \prime} \mathrm{N}$ & $116^{\circ} 17^{\prime} 46^{\prime \prime} \mathrm{W}$ & $\mathbf{L}$ & \\
\hline 5S/7E-30F1 & $\begin{array}{c}77-84 \\
104-126 \\
140-144 \\
180-192\end{array}$ & 66 & $33^{\circ} 42^{\prime} 30^{\prime \prime} \mathrm{N}$ & $116^{\circ} 17^{\prime} 40^{\prime \prime} \mathrm{W}$ & L & \\
\hline $5 \mathrm{~S} / 7 \mathrm{E}-30 \mathrm{~F} 2$ & -- & 67 & $33^{\circ} 42^{\prime} 30^{\prime \prime} \mathrm{N}$ & $116^{\circ} 17^{\prime} 40^{\prime \prime} \mathrm{W}$ & $\mathbf{L}$ & \\
\hline $5 \mathrm{~S} / 7 \mathrm{E}-30 \mathrm{~J} 1$ & $500-900$ & 68 & $33^{\circ} 42^{\prime} 23^{\prime \prime} \mathrm{N}$ & $116^{\circ} 17^{\prime} 08^{\prime \prime} \mathrm{W}$ & LP & \\
\hline $5 \mathrm{~S} / 7 \mathrm{E}-31 \mathrm{~N} 1$ & $110-772$ & 49 & $33^{\circ} 41^{\prime} 09^{\prime \prime} \mathrm{N}$ & $116^{\circ} 17^{\prime} 53^{\prime \prime} \mathrm{W}$ & LP & \\
\hline $5 \mathrm{~S} / 7 \mathrm{E}-31 \mathrm{P} 1$ & $110-680$ & 55 & $33^{\circ} 41^{\prime} 15^{\prime \prime} \mathrm{N}$ & $116^{\circ} 17^{\prime} 51^{\prime \prime} \mathrm{W}$ & LP & \\
\hline $5 S / 7 E-31 Q 1$ & -. & 57 & $33^{\circ} 41^{\prime} 18^{\prime \prime} \mathrm{N}$ & $116^{\circ} 17^{\prime} 34^{\prime \prime} \mathrm{W}$ & $\mathrm{L}$ & \\
\hline $5 \mathrm{~S} / 7 \mathrm{E}-31 \mathrm{Q} 2$ & $\begin{array}{l}380-420 \\
760-920\end{array}$ & 60 & $33^{\circ} 41^{\prime} 12^{\prime \prime} \mathrm{N}$ & $116^{\circ} 17^{\prime} 25^{\prime \prime} \mathrm{W}$ & LP & \\
\hline $5 S / 7 E-32 R 1$ & $180-500$ & 40 & $33^{\circ} 41^{\prime} 18^{\prime \prime} \mathrm{N}$ & $116^{\circ} 16^{\prime} 09^{\prime \prime} \mathrm{W}$ & L & \\
\hline $5 S / 7 E-33 D 2$ & $398-518$ & 43 & $33^{\circ} 41^{\prime} 52^{\prime \prime} \mathrm{N}$ & $116^{\circ} 15^{\prime} 49^{\prime \prime} \mathrm{W}$ & L & \\
\hline $5 S / 7 E-33 F 2$ & $400-540$ & 40 & $33^{\circ} 41^{\prime} 45^{\prime \prime} \mathrm{N}$ & $116^{\circ} 15^{\prime} 40^{\prime \prime} \mathrm{W}$ & $\mathrm{L}$ & \\
\hline $5 \mathrm{~S} / 7 \mathrm{E}-33 \mathrm{~J} 1$ & $500-650$ & 35 & $33^{\circ} 41^{\prime} 32^{\prime \prime} \mathrm{N}$ & $116^{\circ} 15^{\prime} 05^{\prime \prime} \mathrm{W}$ & L & \\
\hline $5 \mathrm{~S} / 7 \mathrm{E}-33 \mathrm{M} 1$ & $388-517$ & 40 & $33^{\circ} 41^{\prime} 30^{\prime \prime} \mathrm{N}$ & $116^{\circ} 15^{\prime} 58^{\prime \prime} \mathrm{W}$ & $\mathbf{L}$ & \\
\hline $5 \mathrm{~S} / 7 \mathrm{E}-34 \mathrm{Q} 2$ & $188-550$ & 5 & $33^{\circ} 41^{\prime} 13^{\prime \prime} \mathrm{N}$ & $116^{\circ} 14^{\prime} 22^{\prime \prime} \mathrm{W}$ & $\mathrm{L}$ & \\
\hline $5 \mathrm{~S} / 7 \mathrm{E}-35 \mathrm{~F} 2$ & $510-800$ & -5 & $33^{\circ} 41^{\prime} 45^{\prime \prime} \mathrm{N}$ & $116^{\circ} 13^{\prime} 40^{\prime \prime} \mathrm{W}$ & L & \\
\hline $5 \mathrm{~S} / 7 \mathrm{E}-36 \mathrm{D} 1$ & $152-756$ & -21 & $33^{\circ} 41^{\prime} 59^{\prime \prime} \mathrm{N}$ & $116^{\circ} 12^{\prime} 53^{\prime \prime} \mathrm{W}$ & $\mathrm{L}$ & \\
\hline $5 S^{\prime} / 7 \mathrm{E}-36 \mathrm{G} 1$ & $125-345$ & -32 & $33^{\circ} 41^{\prime} 44^{\prime \prime} \mathrm{N}$ & $116^{\circ} 12^{\prime} 10^{\prime \prime} \mathrm{W}$ & L & \\
\hline $5 S / 7 E-36 Q 1$ & $147-375$ & -34 & $33^{\circ} 41^{\prime} 20^{\prime \prime} \mathrm{N}$ & $116^{\circ} 12^{\prime} 22^{\prime \prime} \mathrm{W}$ & L & \\
\hline $5 \mathrm{~S} / 8 \mathrm{E}-17 \mathrm{~N} 1$ & $278-398$ & 30 & $33^{\circ} 43^{\prime} 56^{\prime \prime} \mathrm{N}$ & $116^{\circ} 10^{\prime} 45^{\prime \prime} \mathrm{W}$ & $\mathrm{L}$ & \\
\hline $5 \mathrm{~S} / 8 \mathrm{E}-18 \mathrm{H} 1$ & $300-500$ & -25 & $33^{\circ} 44^{\prime} 15^{\prime \prime} \mathrm{N}$ & $116^{\circ} 10^{\prime} 59^{\prime \prime} \mathrm{W}$ & $\overline{\mathrm{L}}$ & \\
\hline $5 S^{\prime} / 8 \mathrm{E}-19 \mathrm{H} 2$ & $402-690$ & 0 & $33^{\circ} 43^{\prime} 29^{\prime \prime} \mathrm{N}$ & $116^{\circ} 10^{\prime} 51^{\prime \prime} \mathrm{W}$ & $\mathrm{L}$ & \\
\hline $5 \mathrm{~S} / 8 \mathrm{E}-20 \mathrm{C2}$ & $278-438$ & -20 & $33^{\circ} 43^{\prime} 41^{\prime \prime} \mathrm{N}$ & $116^{\circ} 10^{\prime} 27^{\prime \prime} \mathrm{W}$ & $\mathrm{L}$ & \\
\hline $5 \mathrm{~S} / 8 \mathrm{E}-20 \mathrm{M} 1$ & $400-450$ & -10 & $33^{\circ} 43^{\prime} 10^{\prime \prime} \mathrm{N}$ & $116^{\circ} 10^{\prime} 36^{\prime \prime} \mathrm{W}$ & $\overline{\mathrm{L}}$ & \\
\hline $5 \mathrm{~S} / 8 \mathrm{E}-28 \mathrm{M} 1$ & $388-460$ & -15 & $33^{\circ} 42^{\prime} 39^{\prime \prime} \mathrm{N}$ & $116^{\circ} 09^{\prime} 37^{\prime \prime} \mathrm{W}$ & $\mathrm{L}$ & \\
\hline $5 \mathrm{~S} / 8 \mathrm{E}-28 \mathrm{M} 2$ & $208-268$ & -40 & $33^{\circ} 42^{\prime} 23^{\prime \prime} \mathrm{N}$ & $116^{\circ} 09^{\prime} 38^{\prime \prime} \mathrm{W}$ & $\mathrm{L}$ & \\
\hline $5 \mathrm{~S} / 8 \mathrm{E}-29 \mathrm{E} 2$ & -- & -30 & $33^{\circ} 42^{\prime} 33^{\prime \prime} \mathrm{N}$ & $116^{\circ} 10^{\prime} 42^{\prime \prime} \mathrm{W}$ & L & \\
\hline $5 \mathrm{~S} / 8 \mathrm{E}-29 \mathrm{G} 1$ & $230-278$ & -28 & $33^{\circ} 42^{\prime} 38^{\prime \prime} \mathrm{N}$ & $116^{\circ} 10^{\prime} 11^{\prime \prime} \mathrm{W}$ & $\bar{L}$ & \\
\hline
\end{tabular}


Appendix 1: List and description of wells in data base--Continued

\begin{tabular}{|c|c|c|c|c|c|c|}
\hline $\begin{array}{l}\text { Well } \\
\text { number }\end{array}$ & $\begin{array}{c}\text { Perforated } \\
\text { interval (depth, in } \\
\text { feet below land } \\
\text { surface) }\end{array}$ & $\begin{array}{c}\text { Land-surface } \\
\text { altitude (feet } \\
\text { above or } \\
\text { below (-) sea } \\
\text { level) }\end{array}$ & Latitude & Longitude & Remarks & $\begin{array}{l}\text { Closest } \\
\text { model node } \\
\text { for pumped } \\
\text { wells (in } \\
\text { model area } \\
\text { only) }\end{array}$ \\
\hline 5S/8E-29R1 & $400-592$ & -50 & $33^{\circ} 42^{\prime} 14^{\prime \prime} \mathrm{N}$ & $116^{\circ} 09^{\prime} 55^{\prime \prime} \mathrm{W}$ & L & \\
\hline $5 \mathrm{~S} / 8 \mathrm{E}-31 \mathrm{C} 3$ & $513-818$ & -40 & $33^{\circ} 41^{\prime} 59^{\prime \prime} \mathrm{N}$ & $116^{\circ} 11^{\prime} 22^{\prime \prime} \mathrm{W}$ & $\mathbf{L}$ & \\
\hline $5 \mathrm{~S} / 8 \mathrm{E}-31 \mathrm{~J} 1$ & $240-302$ & -52 & $33^{\circ} 41^{\prime} 32^{\prime \prime} \mathrm{N}$ & $116^{\circ} 11^{\prime} 05^{\prime \prime} \mathrm{W}$ & $\bar{L}$ & \\
\hline $5 \mathrm{~S} / 8 \mathrm{E}-33 \mathrm{D} 1$ & $521-810$ & -55 & $33^{\circ} 41^{\prime} 54^{\prime \prime} \mathrm{N}$ & $116^{\circ} 09^{\prime} 44^{\prime \prime} \mathrm{W}$ & $\vec{L}$ & \\
\hline $5 \mathrm{~S} / 8 \mathrm{E}-34 \mathrm{G} 1$ & $490-789$ & 25 & $33^{\circ} 41^{\prime} 46^{\prime \prime} \mathrm{N}$ & $116^{\circ} 07^{\prime} 58^{\prime \prime} \mathrm{W}$ & L & \\
\hline $6 \mathrm{~S} / 6 \mathrm{E}-01 \mathrm{G} 1$ & $205-296$ & 50 & $33^{\circ} 40^{\prime} 41^{\prime \prime} \mathrm{N}$ & $116^{\circ} 18^{\prime} 30^{\prime \prime} \mathrm{W}$ & $\vec{L}$ & \\
\hline $6 \mathrm{~S} / 6 \mathrm{E}-01 \mathrm{Q} 1$ & -- & 55 & $33^{\circ} 40^{\prime} 25^{\prime \prime} \mathrm{N}$ & $116^{\circ} 18^{\prime} 27^{\prime \prime} \mathrm{W}$ & LP & \\
\hline 6S/6E-06M1 & $264-364$ & 940 & $33^{\circ} 40^{\prime} 27^{\prime \prime} \mathrm{N}$ & $116^{\circ} 24^{\prime} 21^{\prime \prime} \mathrm{W}$ & $\mathrm{L}$ & \\
\hline $6 \mathrm{~S} / 6 \mathrm{E}-12 \mathrm{~F} 1$ & -- & 118 & $33^{\circ} 40^{\prime} 00^{\prime \prime} \mathrm{N}$ & $116^{\circ} 18^{\prime} 46^{\prime \prime} \mathrm{W}$ & LP & \\
\hline $6 \mathrm{~S} / 6 \mathrm{E}-12 \mathrm{G} 1$ & -- & 90 & $33^{\circ} 40^{\prime} 00^{\prime \prime} \mathrm{N}$ & $116^{\circ} 18^{\prime} 27^{\prime \prime} \mathrm{W}$ & LP & \\
\hline $6 \mathrm{~S} / 6 \mathrm{E}-17 \mathrm{~K} 1$ & $480-580$ & 975 & $33^{\circ} 38^{\prime} 52^{\prime \prime} \mathrm{N}$ & $116^{\circ} 22^{\prime} 41^{\prime \prime} \mathrm{W}$ & $\mathrm{L}$ & \\
\hline $6 \mathrm{~S} / 7 \mathrm{E}-01 \mathrm{H} 1$ & $525-595$ & -45 & $33^{\circ} 40^{\prime} 53^{\prime \prime} \mathrm{N}$ & $116^{\circ} 12^{\prime} 00^{\prime \prime} \mathrm{W}$ & L & \\
\hline $6 \mathrm{~S} / 7 \mathrm{E}-01 \mathrm{P} 1$ & -- & -50 & $33^{\circ} 40^{\prime} 16^{\prime \prime} \mathrm{N}$ & $116^{\circ} 12^{\prime} 38^{\prime \prime} \mathrm{W}$ & $\bar{L}$ & \\
\hline 6S/7E-01Q1 & $460-500$ & -50 & $33^{\circ} 40^{\prime} 18^{\prime \prime} \mathrm{N}$ & $116^{\circ} 12^{\prime} 11^{\prime \prime} \mathrm{W}$ & $\mathrm{L}$ & \\
\hline $6 \mathrm{~S} / 7 \mathrm{E}-02 \mathrm{D} 2$ & -- & -7 & $33^{\circ} 41^{\prime} 01^{\prime \prime} \mathrm{N}$ & $116^{\circ} 13^{\prime} 51^{\prime \prime} \mathrm{W}$ & $\mathrm{L}$ & \\
\hline $6 \mathrm{~S} / 7 \mathrm{E}-02 \mathrm{G} 1$ & $160-363$ & -11 & $33^{\circ} 40^{\prime} 47^{\prime \prime} \mathrm{N}$ & $116^{\circ} 13^{\prime} 25^{\prime \prime} \mathrm{W}$ & $\mathrm{L}$ & \\
\hline $6 \mathrm{~S} / 7 \mathrm{E}-02 \mathrm{~N} 1$ & $426-570$ & -10 & $33^{\circ} 40^{\prime} 27^{\prime \prime} \mathrm{N}$ & $116^{\circ} 13^{\prime} 57^{\prime \prime} \mathrm{W}$ & L & \\
\hline $6 \mathrm{~S} / 7 \mathrm{E}-04 \mathrm{D} 2$ & $472-592$ & -32 & $33^{\circ} 41^{\prime} 03^{\prime \prime} \mathrm{N}$ & $116^{\circ} 15^{\prime} 56^{\prime \prime} \mathrm{W}$ & $\mathrm{L}$ & \\
\hline $6 \mathrm{~S} / 7 \mathrm{E}-04 \mathrm{R} 1$ & -- & 10 & $33^{\circ} 40^{\prime} 21^{\prime \prime} \mathrm{N}$ & $116^{\circ} 15^{\prime} 10^{\prime \prime} \mathrm{W}$ & $\bar{L}$ & \\
\hline $6 \mathrm{~S} / 7 \mathrm{E}-05 \mathrm{~B} 1$ & $368-560$ & 38 & $33^{\circ} 41^{\prime} 03^{\prime \prime} \mathrm{N}$ & $116^{\circ} 16^{\prime} 23^{\prime \prime} \mathrm{W}$ & $\mathrm{L}$ & \\
\hline $6 \mathrm{~S} / 7 \mathrm{E}-06 \mathrm{~B} 1$ & $580-800$ & 48 & $33^{\circ} 40^{\prime} 57^{\prime \prime} \mathrm{N}$ & $116^{\circ} 17^{\prime} 35^{\prime \prime} \mathrm{W}$ & LP & \\
\hline 6S/7E-06N1 & -- & 58 & $33^{\circ} 40^{\prime} 21^{\prime \prime} \mathrm{N}$ & $116^{\circ} 18^{\prime} 24^{\prime \prime} \mathrm{W}$ & LP & \\
\hline 6S/7E-07B1 & $200-480$ & 50 & $33^{\circ} 40^{\prime} 06^{\prime \prime} \mathrm{N}$ & $116^{\circ} 17^{\prime} 35^{\prime \prime} \mathrm{W}$ & $\mathrm{L}$ & \\
\hline $6 \mathrm{~S} / 7 \mathrm{E}-08 \mathrm{D} 2$ & $316-476$ & 31 & $33^{\circ} 40^{\prime} 13^{\prime \prime} \mathrm{N}$ & $116^{\circ} 16^{\prime} 54^{\prime \prime} \mathrm{W}$ & $\overline{\mathrm{L}}$ & \\
\hline $6 \mathrm{~S} / 7 \mathrm{E}-09 \mathrm{~A} 1$ & -- & 8 & $33^{\circ} 40^{\prime} 08^{\prime \prime} \mathrm{N}$ & $116^{\circ} 15^{\prime} 10^{\prime \prime} \mathrm{W}$ & $\mathrm{L}$ & \\
\hline 6S/7E-09L2 & $225-300$ & 9 & $33^{\circ} 39^{\prime} 47^{\prime \prime} \mathrm{N}$ & $116^{\circ} 15^{\prime} 32^{\prime \prime} \mathrm{W}$ & L & \\
\hline $6 \mathrm{~S} / 7 \mathrm{E}-10 \mathrm{G} 1$ & $100-360$ & -15 & $33^{\circ} 39^{\prime} 53^{\prime \prime} \mathrm{N}$ & $116^{\circ} 14^{\prime} 28^{\prime \prime} \mathrm{W}$ & $\mathrm{L}$ & \\
\hline $6 \mathrm{~S} / 7 \mathrm{E}-12 \mathrm{E} 1$ & $120-600$ & -45 & $33^{\circ} 39^{\prime} 51^{\prime \prime} \mathrm{N}$ & $116^{\circ} 12^{\prime} 52^{\prime \prime} \mathrm{W}$ & $\mathrm{L}$ & \\
\hline $6 \mathrm{~S} / 7 \mathrm{E}-12 \mathrm{H} 3$ & $290-350$ & -70 & $33^{\circ} 39^{\prime} 56^{\prime \prime} \mathrm{N}$ & $116^{\circ} 12^{\prime} 24^{\prime \prime} \mathrm{W}$ & $\overrightarrow{\mathrm{L}}$ & \\
\hline $6 \mathrm{~S} / 7 \mathrm{E}-13 \mathrm{M} 2$ & $146-386$ & -56 & $33^{\circ} 38^{\prime} 53^{\prime \prime} \mathrm{N}$ & $116^{\circ} 12^{\prime} 53^{\prime \prime} \mathrm{W}$ & LP & \\
\hline $6 \mathrm{~S} / 7 \mathrm{E}-13 \mathrm{M} 4$ & $480-600$ & -56 & $33^{\circ} 38^{\prime} 51^{\prime \prime} \mathrm{N}$ & $116^{\circ} 12^{\prime} 42^{\prime \prime} \mathrm{W}$ & LP & \\
\hline $6 \mathrm{~S} / 7 \mathrm{E}-16 \mathrm{~A} 2$ & $\begin{array}{l}350-530 \\
650-800\end{array}$ & -5 & $33^{\circ} 39^{\prime} 17^{\prime \prime} \mathrm{N}$ & $116^{\circ} 15^{\prime} 07^{\prime \prime} \mathrm{W}$ & $\mathrm{L}$ & \\
\hline 6S/7E-16D1 & $\begin{array}{l}420-510 \\
550-700\end{array}$ & 7 & $33^{\circ} 39^{\prime} 17^{\prime \prime} \mathrm{N}$ & $116^{\circ} 15^{\prime} 54^{\prime \prime} \mathrm{W}$ & LP & \\
\hline & $1,040-1,110$ & & & & & \\
\hline 6S/7E-17R1 & -- & -5 & $33^{\circ} 38^{\prime} 43^{\prime \prime} \mathrm{N}$ & $116^{\circ} 16^{\prime} 16^{\prime \prime} \mathrm{W}$ & $\mathrm{L}$ & \\
\hline $6 \mathrm{~S} / 7 \mathrm{E}-21 \mathrm{D} 1$ & $\begin{array}{l}550-610 \\
670-800\end{array}$ & -10 & $33^{\circ} 38^{\prime} 24^{\prime \prime} \mathrm{N}$ & $116^{\circ} 15^{\prime} 54^{\prime \prime} \mathrm{W}$ & LP & \\
\hline & $800-1,020$ & & & & & \\
\hline 6S/7E-22B1 & $1,088-1,365$ & -42 & $33^{\circ} 38^{\prime} 30^{\prime \prime} \mathrm{N}$ & $116^{\circ} 14^{\prime} 21^{\prime \prime} \mathrm{W}$ & $\mathrm{L}$ & \\
\hline $6 \mathrm{~S} / 7 \mathrm{E}-23 \mathrm{D} 3$ & $380-600$ & -52 & $33^{\circ} 38^{\prime} 29^{\prime \prime} \mathrm{N}$ & $116^{\circ} 13^{\prime} 42^{\prime \prime} \mathrm{W}$ & $\mathbf{L}$ & \\
\hline $6 \mathrm{~S} / 7 \mathrm{E}-23 \mathrm{~F} 1$ & $312-375$ & -55 & $33^{\circ} 38^{\prime} 17^{\prime \prime} \mathrm{N}$ & $116^{\circ} 13^{\prime} 38^{\prime \prime} \mathrm{W}$ & $\vec{L}$ & \\
\hline $6 \mathrm{~S} / 7 \mathrm{E}-24 \mathrm{D} 5$ & -. & -63 & $33^{\circ} 38^{\prime} 24^{\prime \prime} \mathrm{N}$ & $116^{\circ} 12^{\prime} 47^{\prime \prime} \mathrm{W}$ & $\mathrm{L}$ & \\
\hline 6S/7E-33Q1 & -- & 90 & $33^{\circ} 35^{\prime} 59^{\prime \prime} \mathrm{N}$ & $116^{\circ} 15^{\prime} 22^{\prime \prime} \mathrm{W}$ & $\mathrm{L}$ & \\
\hline 6S/7E-33R1 & -- & 70 & $33^{\circ} 35^{\prime} 59^{\prime \prime} \mathrm{N}$ & $116^{\circ} 15^{\prime} 07^{\prime \prime} \mathrm{W}$ & $\mathrm{L}$ & \\
\hline $6 \mathrm{~S} / 7 \mathrm{E}-33 \mathrm{R} 2$ & -- & 80 & $33^{\circ} 35^{\prime} 58^{\prime \prime} \mathrm{N}$ & $116^{\circ} 15^{\prime} 07^{\prime \prime} \mathrm{W}$ & $\mathrm{L}$ & \\
\hline $6 \mathrm{~S} / 7 \mathrm{E}-33 \mathrm{R} 3$ & -- & 75 & $33^{\circ} 35^{\prime} 58^{\prime \prime} \mathrm{N}$ & $116^{\circ} 15^{\prime} 07^{\prime \prime} \mathrm{W}$ & $\mathrm{L}$ & \\
\hline $6 \mathrm{~S} / 8 \mathrm{E}-02 \mathrm{D} 1$ & $292-760$ & 9 & $33^{\circ} 41^{\prime} 00^{\prime \prime} \mathrm{N}$ & $116^{\circ} 07^{\prime} 35^{\prime \prime} \mathrm{W}$ & $\mathrm{L}$ & \\
\hline 6S/8E-02F1 & $540-1,015$ & 5 & $33^{\circ} 40^{\prime} 54^{\prime \prime} \mathrm{N}$ & $116^{\circ} 07^{\prime} 13^{\prime \prime} \mathrm{W}$ & $\mathrm{L}$ & \\
\hline $6 \mathrm{~S} / 8 \mathrm{E}-03 \mathrm{C} 1$ & $508-1,140$ & -69 & $33^{\circ} 41^{\prime} 06^{\prime \prime} \mathrm{N}$ & $116^{\circ} 08^{\prime} 15^{\prime \prime} \mathrm{W}$ & L & \\
\hline 6S/8E-03D1 & -- & -84 & $33^{\circ} 41^{\prime} 01^{\prime \prime} \mathrm{N}$ & $116^{\circ} 08^{\prime} 37^{\prime \prime} \mathrm{W}$ & $\mathbf{L}$ & \\
\hline $6 \mathrm{~S} / 8 \mathrm{E}-05 \mathrm{P} 1$ & $216-264$ & -75 & $33^{\circ} 40^{\prime} 17^{\prime \prime} \mathrm{N}$ & $116^{\circ} 10^{\prime} 33^{\prime \prime} \mathrm{W}$ & $\bar{L}$ & \\
\hline $6 \mathrm{~S} / 8 \mathrm{E}-05 \mathrm{R} 1$ & $206-640$ & -80 & $33^{\circ} 40^{\prime} 27^{\prime \prime} \mathrm{N}$ & $116^{\circ} 10^{\prime} 02^{\prime \prime} \mathrm{W}$ & LP & \\
\hline
\end{tabular}


Appendix 1: List and description of wells in data base--Continued

\begin{tabular}{|c|c|c|c|c|c|c|}
\hline $\begin{array}{l}\text { Well } \\
\text { number }\end{array}$ & $\begin{array}{c}\text { Perforated } \\
\text { interval (depth, in } \\
\text { feet below land } \\
\text { surface) }\end{array}$ & $\begin{array}{l}\text { Land-surface } \\
\text { altitude (feet } \\
\text { above or } \\
\text { below (-) sea } \\
\text { level) }\end{array}$ & Latitude & Longitude & Remarks & $\begin{array}{l}\text { Closest } \\
\text { model node } \\
\text { for pumped } \\
\text { wells (in } \\
\text { model area } \\
\text { only) }\end{array}$ \\
\hline 6S/8E-05R2 & $540-750$ & -82 & $33^{\circ} 40^{\prime} 26^{\prime \prime} \mathrm{N}$ & $116^{\circ} 09^{\prime} 54^{\prime \prime} \mathrm{W}$ & LP & \\
\hline $6 \mathrm{~S} / 8 \mathrm{E}-05 \mathrm{R} 3$ & $520-650$ & -80 & $33^{\circ} 40^{\prime} 28^{\prime \prime} \mathrm{N}$ & $116^{\circ} 10^{\prime} 00^{\prime \prime} \mathrm{W}$ & LP & \\
\hline $6 \mathrm{~S} / 8 \mathrm{E}-06 \mathrm{G3}$ & $200-260$ & -62 & $33^{\circ} 40^{\prime} 42^{\prime \prime} \mathrm{N}$ & $116^{\circ} 11^{\prime} 06^{\prime \prime} \mathrm{W}$ & $\mathrm{L}$ & \\
\hline $6 \mathrm{~S} / 8 \mathrm{E}-06 \mathrm{M} 1$ & -- & -55 & $33^{\circ} 40^{\prime} 35^{\prime \prime} \mathrm{N}$ & $116^{\circ} 11^{\prime} 45^{\prime \prime} \mathrm{W}$ & $\mathrm{L}$ & \\
\hline $6 \mathrm{~S} / 8 \mathrm{E}-08 \mathrm{~L} 1$ & -- & -85 & $33^{\circ} 39^{\prime} 43^{\prime \prime} \mathrm{N}$ & $116^{\circ} 10^{\prime} 27^{\prime \prime} \mathrm{W}$ & $L$ & \\
\hline $6 \mathrm{~S}^{\prime} / 8 \mathrm{E}-09 \mathrm{~K} 2$ & $468-548$ & -98 & $33^{\circ} 39^{\prime} 41^{\prime \prime} \mathrm{N}$ & $116^{\circ} 09^{\prime} 01^{\prime \prime} \mathrm{W}$ & L & \\
\hline $6 \mathrm{~S} / 8 \mathrm{E}-09 \mathrm{Q} 2$ & $620-1,025$ & -102 & $33^{\circ} 39^{\prime} 24^{\prime \prime} \mathrm{N}$ & $116^{\circ} 09^{\prime} 14^{\prime \prime} \mathrm{W}$ & $\mathbf{L}$ & \\
\hline $6 \mathrm{~S} / 8 \mathrm{E}-09 \mathrm{Q} 4$ & $580-680$ & -102 & $33^{\circ} 39^{\prime} 25^{\prime \prime} \mathrm{N}$ & $116^{\circ} 09^{\prime} 15^{\prime \prime} \mathrm{W}$ & $\mathbf{L}$ & \\
\hline $6 \mathrm{~S} / 8 \mathrm{E}-10 \mathrm{~F} 1$ & $506-583$ & -99 & $33^{\circ} 40^{\prime} 01^{\prime \prime} \mathrm{N}$ & $116^{\circ} 08^{\prime} 17^{\prime \prime} \mathrm{W}$ & $\mathbf{L}$ & \\
\hline $6 \mathrm{~S} / 8 \mathrm{E}-11 \mathrm{~A} 1$ & $400-842$ & -25 & $33^{\circ} 40^{\prime} 08^{\prime \prime} \mathrm{N}$ & $116^{\circ} 06^{\prime} 48^{\prime \prime} \mathrm{W}$ & L & \\
\hline $6 \mathrm{~S} / 8 \mathrm{E}-11 \mathrm{E} 1$ & -- & -100 & $33^{\circ} 40^{\prime} 01^{\prime \prime} \mathrm{N}$ & $116^{\circ} 07^{\prime} 35^{\prime \prime} \mathrm{W}$ & $\mathbf{L}$ & \\
\hline 6S/8E-17R1 & $470-550$ & -109 & $33^{\circ} 38^{\prime} 34^{\prime \prime} \mathrm{N}$ & $116^{\circ} 10^{\prime} 01^{\prime \prime} \mathrm{W}$ & L & \\
\hline 6S/8E-19D1 & $1,196-1,413$ & -85 & $33^{\circ} 38^{\prime} 29^{\prime \prime} \mathrm{N}$ & $116^{\circ} 11^{\prime} 47^{\prime \prime} \mathrm{W}$ & L & \\
\hline $6 \mathrm{~S} / 8 \mathrm{E}-19 \mathrm{D} 2$ & $450-570$ & -87 & $33^{\circ} 38^{\prime} 29^{\prime \prime} \mathrm{N}$ & $116^{\circ} 11^{\prime} 39^{\prime \prime} \mathrm{W}$ & $\mathbf{L}$ & \\
\hline $6 \mathrm{~S} / 8 \mathrm{E}-19 \mathrm{R} 1$ & -- & -105 & $33^{\circ} 37^{\prime} 49^{\prime \prime} \mathrm{N}$ & $116^{\circ} 10^{\prime} 59^{\prime \prime} \mathrm{W}$ & $L$ & \\
\hline $6 \mathrm{~S} / 8 \mathrm{E}-20 \mathrm{H} 1$ & $430-670$ & -117 & $33^{\circ} 38^{\prime} 09^{\prime \prime} \mathrm{N}$ & $116^{\circ} 09^{\prime} 57^{\prime \prime} \mathrm{W}$ & LP & \\
\hline $6 \mathrm{~S} / 8 \mathrm{E}-22 \mathrm{D} 2$ & $\begin{array}{c}500-680 \\
900-1,100\end{array}$ & -120 & $33^{\circ} 38^{\prime} 25^{\prime \prime} \mathrm{N}$ & $116^{\circ} 08^{\prime} 31^{\prime \prime} \mathrm{W}$ & LP & \\
\hline $6 \mathrm{~S} / 8 \mathrm{E}-22 \mathrm{~K} 1$ & $500-1,030$ & -128 & $33^{\circ} 38^{\prime} 02^{\prime \prime} \mathrm{N}$ & $116^{\circ} 08^{\prime} 12^{\prime \prime} \mathrm{W}$ & $\mathbf{L}$ & \\
\hline $6 \mathrm{~S} / 8 \mathrm{E}-25 \mathrm{P} 1$ & $478-658$ & -140 & $33^{\circ} 36^{\prime} 46^{\prime \prime} \mathrm{N}$ & $116^{\circ} 06^{\prime} 16^{\prime \prime} \mathrm{W}$ & $\vec{L}$ & \\
\hline $6 \mathrm{~S} / 8 \mathrm{E}-27 \mathrm{C1}$ & $670-1,070$ & -135 & $33^{\circ} 37^{\prime} 38^{\prime \prime} \mathrm{N}$ & $116^{\circ} 08^{\prime} 14^{\prime \prime} \mathrm{W}$ & $\mathrm{L}$ & \\
\hline $6 \mathrm{~S} / 8 \mathrm{E}-27 \mathrm{~N} 1$ & $312-438$ & -145 & $33^{\circ} 36^{\prime} 46^{\prime \prime} \mathrm{N}$ & $116^{\circ} 08^{\prime} 36^{\prime \prime} \mathrm{W}$ & $\mathrm{L}$ & \\
\hline $6 \mathrm{~S} / 8 \mathrm{E}-32 \mathrm{R} 1$ & -- & -140 & $33^{\circ} 36^{\prime} 05^{\prime \prime} \mathrm{N}$ & $116^{\circ} 10^{\prime} 02^{\prime \prime} \mathrm{W}$ & L & \\
\hline $6 \mathrm{~S} / 8 \mathrm{E}-34 \mathrm{C} 1$ & $447-545$ & -146 & $33^{\circ} 36^{\prime} 44^{\prime \prime} \mathrm{N}$ & $116^{\circ} 08^{\prime} 13^{\prime \prime} \mathrm{W}$ & $\mathbf{L}$ & \\
\hline $6 \mathrm{~S} / 8 \mathrm{E}-35 \mathrm{~J} 1$ & 514-564 & -155 & $33^{\circ} 36^{\prime} 16^{\prime \prime} \mathrm{N}$ & $116^{\circ} 06^{\prime} 58^{\prime \prime} \mathrm{W}$ & $\mathrm{L}$ & \\
\hline $6 \mathrm{~S} / 8 \mathrm{E}-36 \mathrm{M} 1$ & $1,540-1,880$ & -155 & $33^{\circ} 36^{\prime} 17^{\prime \prime} \mathrm{N}$ & $116^{\circ} 06^{\prime} 42^{\prime \prime} \mathrm{W}$ & $\bar{L}$ & \\
\hline 6S/9E-19L1 & $430-730$ & -35 & $33^{\circ} 37^{\prime} 54^{\prime \prime} \mathrm{N}$ & $116^{\circ} 05^{\prime} 10^{\prime \prime} \mathrm{W}$ & $\mathrm{L}$ & \\
\hline $6 \mathrm{~S} / 9 \mathrm{E}-30 \mathrm{~A} 1$ & $220-355$ & -51 & $33^{\circ} 37^{\prime} 38^{\prime \prime} \mathrm{N}$ & $116^{\circ} 04^{\prime} 53^{\prime \prime} \mathrm{W}$ & $\mathrm{L}$ & \\
\hline $6 \mathrm{~S} / 9 \mathrm{E}-32 \mathrm{~A} 1$ & $218-598$ & 20 & $33^{\circ} 36^{\prime} 45^{\prime \prime} \mathrm{N}$ & $116^{\circ} 03^{\prime} 39^{\prime \prime} \mathrm{W}$ & $\mathrm{L}$ & \\
\hline $6 \mathrm{~S} / 9 \mathrm{E}-32 \mathrm{Q} 1$ & $244-284$ & -100 & $33^{\circ} 36^{\prime} 00^{\prime \prime} \mathrm{N}$ & $116^{\circ} 03^{\prime} 57^{\prime \prime} \mathrm{W}$ & $L$ & \\
\hline $6 \mathrm{~S} / 9 \mathrm{E}-33 \mathrm{~K} 1$ & $240-402$ & 40 & $33^{\circ} 36^{\prime} 14^{\prime \prime} \mathrm{N}$ & $116^{\circ} 02^{\prime} 53^{\prime \prime} \mathrm{W}$ & L & \\
\hline $7 \mathrm{~S} / 7 \mathrm{E}-01 \mathrm{C} 1$ & $240-380$ & -112 & $33^{\circ} 35^{\prime} 49^{\prime \prime} \mathrm{N}$ & $116^{\circ} 12^{\prime} 24^{\prime \prime} \mathrm{W}$ & $L$ & \\
\hline 7S/7E-01M1 & -- & -111 & $33^{\circ} 35^{\prime} 25^{\prime \prime} \mathrm{N}$ & $116^{\circ} 12^{\prime} 52^{\prime \prime} \mathrm{W}$ & $\mathbf{L}$ & \\
\hline $7 \mathrm{~S} / 7 \mathrm{E}-02 \mathrm{H} 1$ & $278-326$ & -105 & $33^{\circ} 35^{\prime} 37^{\prime \prime} \mathrm{N}$ & $116^{\circ} 13^{\prime} 10^{\prime \prime} \mathrm{W}$ & $\mathbf{L}$ & \\
\hline $7 \mathrm{~S} / 7 \mathrm{E}-03 \mathrm{~A} 1$ & $250-452$ & -72 & $33^{\circ} 35^{\prime} 44^{\prime \prime} \mathrm{N}$ & $116^{\circ} 14^{\prime} 03^{\prime \prime} \mathrm{W}$ & $\mathbf{L}$ & \\
\hline $7 \mathrm{~S} / 8 \mathrm{E}-02 \mathrm{~B} 1$ & $520-575$ & -161 & $33^{\circ} 35^{\prime} 50^{\prime \prime} \mathrm{N}$ & $116^{\circ} 07^{\prime} 02^{\prime \prime} \mathrm{W}$ & $\overline{\mathrm{L}}$ & \\
\hline $7 \mathrm{~S} / 8 \mathrm{E}-03 \mathrm{~A} 1$ & $400-500$ & -159 & $33^{\circ} 35^{\prime} 52^{\prime \prime} \mathrm{N}$ & $116^{\circ} 07^{\prime} 55^{\prime \prime} \mathrm{W}$ & $\mathbf{L}$ & \\
\hline $7 \mathrm{~S} / 8 \mathrm{E}-07 \mathrm{R} 1$ & -- & -90 & $33^{\circ} 34^{\prime} 09^{\prime \prime} \mathrm{N}$ & $116^{\circ} 10^{\prime} 53^{\prime \prime} \mathrm{W}$ & $\mathbf{L}$ & \\
\hline $7 \mathrm{~S} / 8 \mathrm{E}-08 \mathrm{~N} 1$ & $300-360$ & -92 & $33^{\circ} 34^{\prime} 09^{\prime \prime} \mathrm{N}$ & $116^{\circ} 10^{\prime} 46^{\prime \prime} \mathrm{W}$ & L & \\
\hline $7 \mathrm{~S} / 8 \mathrm{E}-09 \mathrm{M} 1$ & $540-600$ & -147 & $33^{\circ} 34^{\prime} 21^{\prime \prime} \mathrm{N}$ & $116^{\circ} 09^{\prime} 48^{\prime \prime} \mathrm{W}$ & $\mathbf{L}$ & \\
\hline $7 \mathrm{~S} / 8 \mathrm{E}-15 \mathrm{P} 1$ & $310-1,060$ & -140 & $33^{\circ} 33^{\prime} 17^{\prime \prime} \mathrm{N}$ & $116^{\circ} 08^{\prime} 32^{\prime \prime} \mathrm{W}$ & $\mathbf{L}$ & \\
\hline $7 \mathrm{~S} / 8 \mathrm{E}-17 \mathrm{~A} 1$ & $800-1,100$ & -118 & $33^{\circ} 34^{\prime} 01^{\prime \prime} \mathrm{N}$ & $116^{\circ} 09^{\prime} 58^{\prime \prime} \mathrm{W}$ & $\mathrm{L}$ & \\
\hline $7 \mathrm{~S} / 8 \mathrm{E}-17 \mathrm{~F} 1$ & $265-325$ & -79 & $33^{\circ} 33^{\prime} 47^{\prime \prime} \mathrm{N}$ & $116^{\circ} 10^{\prime} 22^{\prime \prime} \mathrm{W}$ & $L$ & \\
\hline $7 \mathrm{~S} / 8 \mathrm{E}-17 \mathrm{G} 1$ & $400-750$ & -78 & $33^{\circ} 33^{\prime} 44^{\prime \prime} \mathrm{N}$ & $116^{\circ} 10^{\prime} 14^{\prime \prime} \mathrm{W}$ & LP & \\
\hline $7 \mathrm{~S} / 8 \mathrm{E}-18 \mathrm{C} 1$ & -- & .73 & $33^{\circ} 34^{\prime} 03^{\prime \prime} \mathrm{N}$ & $116^{\circ} 11^{\prime} 36^{\prime \prime} \mathrm{W}$ & $L$ & \\
\hline $7 \mathrm{~S} / 8 \mathrm{E}-18 \mathrm{Q} 1$ & $300-500$ & 2 & $33^{\circ} 33^{\prime} 16^{\prime \prime} \mathrm{N}$ & $116^{\circ} 11^{\prime} 23^{\prime \prime} \mathrm{W}$ & $\mathbf{L}$ & \\
\hline $7 \mathrm{~S} / 8 \mathrm{E}-19 \mathrm{G} 1$ & -- & 7 & $33^{\circ} 32^{\prime} 56^{\prime \prime} \mathrm{N}$ & $116^{\circ} 11^{\prime} 14^{\prime \prime} \mathrm{W}$ & $\mathbf{L}$ & \\
\hline $7 \mathrm{~S} / 8 \mathrm{E}-20 \mathrm{~B} 1$ & $210-501$ & -2 & $33^{\circ} 33^{\prime} 04^{\prime \prime} \mathrm{N}$ & $116^{\circ} 10^{\prime} 20^{\prime \prime} \mathrm{W}$ & $\mathrm{L}$ & \\
\hline $7 \mathrm{~S} / 8 \mathrm{E}-20 \mathrm{H} 1$ & $260-486$ & -22 & $33^{\circ} 32^{\prime} 53^{\prime \prime} \mathrm{N}$ & $116^{\circ} 09^{\prime} 52^{\prime \prime} \mathrm{W}$ & L & \\
\hline $7 \mathrm{~S} / 8 \mathrm{E}-22 \mathrm{~K} 1$ & $446-775$ & -124 & $33^{\circ} 32^{\prime} 39^{\prime \prime} \mathrm{N}$ & $116^{\circ} 08^{\prime} 17^{\prime \prime} \mathrm{W}$ & L & \\
\hline $7 \mathrm{~S} / 8 \mathrm{E}-23 \mathrm{Q} 1$ & $316-416$ & -181 & $33^{\circ} 32^{\prime} 24^{\prime \prime} \mathrm{N}$ & $116^{\circ} 07^{\prime} 03^{\prime \prime} \mathrm{W}$ & $\bar{L}$ & \\
\hline $7 \mathrm{~S} / 8 \mathrm{E}-23 \mathrm{Q} 2$ & $365-425$ & -171 & $33^{\circ} 32^{\prime} 24^{\prime \prime} \mathrm{N}$ & $116^{\circ} 07^{\prime} 15^{\prime \prime} \mathrm{W}$ & $\mathrm{L}$ & \\
\hline $7 \mathrm{~S} / 8 \mathrm{E}-28 \mathrm{G} 1$ & $195-295$ & -16 & $33^{\circ} 31^{\prime} 59^{\prime \prime} \mathrm{N}$ & $116^{\circ} 09^{\prime} 14^{\prime \prime} \mathrm{W}$ & $\mathbf{L}$ & \\
\hline $7 S / 8 E-29 D 1$ & $360-650$ & 95 & $33^{\circ} 32^{\prime} 16^{\prime \prime} \mathrm{N}$ & $116^{\circ} 10^{\prime} 45^{\prime \prime} \mathrm{W}$ & $\mathbf{L}$ & \\
\hline
\end{tabular}


Appendix 1: List and description of wells in data base--Continued

\begin{tabular}{|c|c|c|c|c|c|c|}
\hline $\begin{array}{l}\text { Well } \\
\text { number }\end{array}$ & $\begin{array}{l}\text { Perforated } \\
\text { interval (depth, in } \\
\text { feet below land } \\
\text { surface) }\end{array}$ & $\begin{array}{l}\text { Land-surface } \\
\text { altitude (feet } \\
\text { above or } \\
\text { below (-) sea } \\
\text { level) }\end{array}$ & Latitude & Longitude & Remarks & $\begin{array}{l}\text { Closest } \\
\text { model node } \\
\text { for pumped } \\
\text { wells (in } \\
\text { model area } \\
\text { only) }\end{array}$ \\
\hline 7S/8E-29G1 & -- & 80 & $33^{\circ} 32^{\prime} 03^{\prime \prime} \mathrm{N}$ & $116^{\circ} 10^{\prime} 14^{\prime \prime} \mathrm{W}$ & $\mathbf{L}$ & \\
\hline $7 \mathrm{~S} / 8 \mathrm{E}-31 \mathrm{R} 1$ & -- & 24 & $33^{\circ} 30^{\prime} 46^{\prime \prime} \mathrm{N}$ & $116^{\circ} 10^{\prime} 58^{\prime \prime} \mathrm{W}$ & $\mathbf{L}$ & \\
\hline $7 \mathrm{~S} / 8 \mathrm{E}-33 \mathrm{~B} 1$ & $243-522$ & 21 & $33^{\circ} 31^{\prime} 18^{\prime \prime} \mathrm{N}$ & $116^{\circ} 09^{\prime} 17^{\prime \prime} \mathrm{W}$ & $\mathrm{L}$ & \\
\hline $7 \mathrm{~S} / 8 \mathrm{E}-33 \mathrm{E} 1$ & $318-702$ & 75 & $33^{\circ} 31^{\prime} 17^{\prime \prime} \mathrm{N}$ & $116^{\circ} 09^{\prime} 49^{\prime \prime} \mathrm{W}$ & $\mathrm{L}$ & \\
\hline $7 \mathrm{~S} / 8 \mathrm{E}-33 \mathrm{~N} 2$ & - & 75 & $33^{\circ} 30^{\prime} 45^{\prime \prime} \mathrm{N}$ & $116^{\circ} 09^{\prime} 43^{\prime \prime} \mathrm{W}$ & $\mathrm{L}$ & \\
\hline $7 \mathrm{~S} / 8 \mathrm{E}-34 \mathrm{G} 1$ & -- & -92 & $33^{\circ} 31^{\prime} 10^{\prime \prime} \mathrm{N}$ & $116^{\circ} 08^{\prime} 07^{\prime \prime} \mathrm{W}$ & $\mathrm{L}$ & \\
\hline $7 \mathrm{~S} / 8 \mathrm{E}-34 \mathrm{~K} 1$ & $300-895$ & -84 & $33^{\circ} 30^{\prime} 55^{\prime \prime} \mathrm{N}$ & $116^{\circ} 08^{\prime} 12^{\prime \prime} \mathrm{W}$ & $\bar{L}$ & \\
\hline $7 \mathrm{~S} / 8 \mathrm{E}-35 \mathrm{~K} 1$ & -- & -161 & $33^{\circ} 30^{\prime} 52^{\prime \prime} \mathrm{N}$ & $116^{\circ} 07^{\prime} 14^{\prime \prime} \mathrm{W}$ & $\mathrm{L}$ & \\
\hline $7 \mathrm{~S} / 9 \mathrm{E}-03 \mathrm{D} 1$ & $320-600$ & 31 & $33^{\circ} 35^{\prime} 48^{\prime \prime} \mathrm{N}$ & $116^{\circ} 02^{\prime} 27^{\prime \prime} \mathrm{W}$ & $\mathrm{L}$ & \\
\hline $7 \mathrm{~S} / 9 \mathrm{E}-04 \mathrm{C} 1$ & $300-600$ & -42 & $33^{\circ} 35^{\prime} 50^{\prime \prime} \mathrm{N}$ & $116^{\circ} 03^{\prime} 15^{\prime \prime} \mathrm{W}$ & $\mathrm{L}$ & \\
\hline $7 \mathrm{~S} / 9 \mathrm{E}-04 \mathrm{~K} 1$ & $300-600$ & -65 & $33^{\circ} 35^{\prime} 17^{\prime \prime} \mathrm{N}$ & $116^{\circ} 03^{\prime} 06^{\prime \prime} \mathrm{W}$ & $\vec{L}$ & \\
\hline $7 \mathrm{~S} / 9 \mathrm{E}-05 \mathrm{M} 1$ & $460-940$ & -152 & $33^{\circ} 35^{\prime} 24^{\prime \prime} \mathrm{N}$ & $116^{\circ} 04^{\prime} 26^{\prime \prime} \mathrm{W}$ & $\mathbf{L}$ & \\
\hline $7 \mathrm{~S} / 9 \mathrm{E}-07 \mathrm{H} 2$ & $451-566$ & -188 & $33^{\circ} 34^{\prime} 37^{\prime \prime} \mathrm{N}$ & $116^{\circ} 04^{\prime} 57^{\prime \prime} \mathrm{W}$ & L & \\
\hline \multirow[t]{2}{*}{$7 \mathrm{~S} / 9 \mathrm{E}-07 \mathrm{~J} 1$} & $\begin{array}{l}410-470 \\
500-570\end{array}$ & -185 & $33^{\circ} 34^{\prime} 31^{\prime \prime} \mathrm{N}$ & $116^{\circ} 04^{\prime} 46^{\prime \prime} \mathrm{W}$ & LP & \\
\hline & $830-870$ & & & & & \\
\hline 7S/9E-08P1 & $430-590$ & -180 & $33^{\circ} 34^{\prime} 16^{\prime \prime} \mathrm{N}$ & $116^{\circ} 04^{\prime} 13^{\prime \prime} \mathrm{W}$ & LP & \\
\hline $7 \mathrm{~S} / 9 \mathrm{E}-13 \mathrm{~N} 1$ & $90-306$ & -101 & $33^{\circ} 33^{\prime} 19^{\prime \prime} \mathrm{N}$ & $116^{\circ} 00^{\prime} 28^{\prime \prime} \mathrm{W}$ & L & \\
\hline $7 \mathrm{~S} / 9 \mathrm{E}-16 \mathrm{M} 2$ & $530-590$ & -186 & $33^{\circ} 33^{\prime} 34^{\prime \prime} \mathrm{N}$ & $116^{\circ} 03^{\prime} 29^{\prime \prime} W$ & $\mathrm{~L}$ & \\
\hline $7 \mathrm{~S} / 9 \mathrm{E}-17 \mathrm{~K} 1$ & $\begin{array}{l}420-570 \\
590-620\end{array}$ & -195 & $33^{\circ} 33^{\prime} 41^{\prime \prime} \mathrm{N}$ & $116^{\circ} 04^{\prime} 05^{\prime \prime} \mathrm{W}$ & LP & \\
\hline & $\begin{array}{l}530-0<0 \\
830-900\end{array}$ & & & & & \\
\hline $7 \mathrm{~S} / 9 \mathrm{E}-22 \mathrm{G} 2$ & $560-620$ & -173 & $33^{\circ} 32^{\prime} 56^{\prime \prime} \mathrm{N}$ & $116^{\circ} 01^{\prime} 52^{\prime \prime} \mathrm{W}$ & $\mathbf{L}$ & \\
\hline $7 \mathrm{~S} / 9 \mathrm{E}-23 \mathrm{~N} 1$ & $530-560$ & -187 & $33^{\circ} 32^{\prime} 25^{\prime \prime} \mathrm{N}$ & $116^{\circ} 01^{\prime} 31^{\prime \prime} \mathrm{W}$ & LP & \\
\hline $7 \mathrm{~S} / 9 \mathrm{E}-26 \mathrm{G} 2$ & $336-432$ & -205 & $33^{\circ} 31^{\prime} 59^{\prime \prime} \mathrm{N}$ & $116^{\circ} 01^{\prime} 00^{\prime \prime} \mathrm{W}$ & $\mathbf{L}$ & \\
\hline $7 \mathrm{~S} / 9 \mathrm{E}-30 \mathrm{M} 1$ & -- & -213 & $33^{\circ} 31^{\prime} 58^{\prime \prime} \mathrm{N}$ & $116^{\circ} 05^{\prime} 37^{\prime \prime} \mathrm{W}$ & L & \\
\hline $7 \mathrm{~S} / 10 \mathrm{E}-27 \mathrm{~A} 1$ & -- & 34 & $33^{\circ} 32^{\prime} 18^{\prime \prime} \mathrm{N}$ & $115^{\circ} 55^{\prime} 24^{\prime \prime} \mathrm{W}$ & $\bar{L}$ & \\
\hline $8 \mathrm{~S} / 8 \mathrm{E}-03 \mathrm{~B} 1$ & $485-680$ & -100 & $33^{\circ} 30^{\prime} 34^{\prime \prime} \mathrm{N}$ & $116^{\circ} 08^{\prime} 02^{\prime \prime} \mathrm{W}$ & $\bar{L}$ & \\
\hline $8 \mathrm{~S} / 8 \mathrm{E}-03 \mathrm{~L} 1$ & $402-618$ & -59 & $33^{\circ} 30^{\prime} 11^{\prime \prime} \mathrm{N}$ & $116^{\circ} 08^{\prime} 29^{\prime \prime} \mathrm{W}$ & L & \\
\hline $8 \mathrm{~S} / 8 \mathrm{E}-05 \mathrm{H1}$ & $250-560$ & 9 & $33^{\circ} 30^{\prime} 19^{\prime \prime} \mathrm{N}$ & $116^{\circ} 09^{\prime} 58^{\prime \prime} \mathrm{W}$ & $\mathrm{L}$ & \\
\hline $8 \mathrm{~S} / 8 \mathrm{E}-11 \mathrm{~A} 4$ & $300-380$ & -157 & $33^{\circ} 29^{\prime} 48^{\prime \prime} \mathrm{N}$ & $116^{\circ} 06^{\prime} 50^{\prime \prime} \mathrm{W}$ & $\mathrm{L}$ & \\
\hline $8 \mathrm{~S} / 8 \mathrm{E}-11 \mathrm{H} 1$ & $560-876$ & -166 & $33^{\circ} 29^{\prime} 36^{\prime \prime} \mathrm{N}$ & $116^{\circ} 06^{\prime} 51^{\prime \prime} \mathrm{W}$ & $\overrightarrow{\mathrm{L}}$ & \\
\hline $8 \mathrm{~S} / 8 \mathrm{E}-15 \mathrm{G} 1$ & .. & 55 & $33^{\circ} 28^{\prime} 43^{\prime \prime} \mathrm{N}$ & $116^{\circ} 08^{\prime} 09^{\prime \prime} \mathrm{W}$ & $\mathrm{L}$ & \\
\hline $8 \mathrm{~S} / 8 \mathrm{E}-24 \mathrm{~A} 1$ & $800-900$ & -155 & $33^{\circ} 28^{\prime} 04^{\prime \prime} \mathrm{N}$ & $116^{\circ} 05^{\prime} 48^{\prime \prime} \mathrm{W}$ & $\mathrm{L}$ & \\
\hline $8 \mathrm{~S} / 8 \mathrm{E}-24 \mathrm{~A} 2$ & -. & -154 & $33^{\circ} 28^{\prime} 01^{\prime \prime} \mathrm{N}$ & $116^{\circ} 05^{\prime} 51^{\prime \prime} \mathrm{W}$ & $\mathrm{L}$ & \\
\hline $8 \mathrm{~S} / 8 \mathrm{E}-24 \mathrm{~J} 1$ & $216-312$ & -148 & $33^{\circ} 27^{\prime} 43^{\prime \prime} \mathrm{N}$ & $116^{\circ} 05^{\prime} 41^{\prime \prime} \mathrm{W}$ & $\mathrm{L}$ & \\
\hline $8 \mathrm{~S} / 8 \mathrm{E}-24 \mathrm{~L} 1$ & - & -110 & $33^{\circ} 27^{\prime} 37^{\prime \prime} \mathrm{N}$ & $116^{\circ} 06^{\prime} 19^{\prime \prime} \mathrm{W}$ & $\mathrm{L}$ & \\
\hline $8 \mathrm{~S} / 9 \mathrm{E}-30 \mathrm{~A} 1$ & $315-595$ & -152 & $33^{\circ} 27^{\prime} 08^{\prime \prime} \mathrm{N}$ & $116^{\circ} 04^{\prime} 54^{\prime \prime} \mathrm{W}$ & $\mathrm{L}$ & \\
\hline $8 \mathrm{~S} / 9 \mathrm{E}-31 \mathrm{Q} 1$ & $230-350$ & -6 & $33^{\circ} 25^{\prime} 35^{\prime \prime} \mathrm{N}$ & $116^{\circ} 04^{\prime} 59^{\prime \prime} \mathrm{W}$ & $\mathbf{L P}$ & \\
\hline 8S/9E-31R1 & $182-278$ & -17 & $33^{\circ} 25^{\prime} 35^{\prime \prime} \mathrm{N}$ & $116^{\circ} 04^{\prime} 41^{\prime \prime} \mathrm{W}$ & LP & \\
\hline 8S/9E-31R2 & $180-348$ & -18 & $33^{\circ} 25^{\prime} 36^{\prime \prime} \mathrm{N}$ & $116^{\circ} 04^{\prime} 44^{\prime \prime} \mathrm{W}$ & LP & \\
\hline $8 \mathrm{~S} / 9 \mathrm{E}-33 \mathrm{~N} 1$ & - & -133 & $33^{\circ} 25^{\prime} 36^{\prime \prime} \mathrm{N}$ & $116^{\circ} 03^{\prime} 33^{\prime \prime}$ W & $\mathrm{L}$ & \\
\hline
\end{tabular}




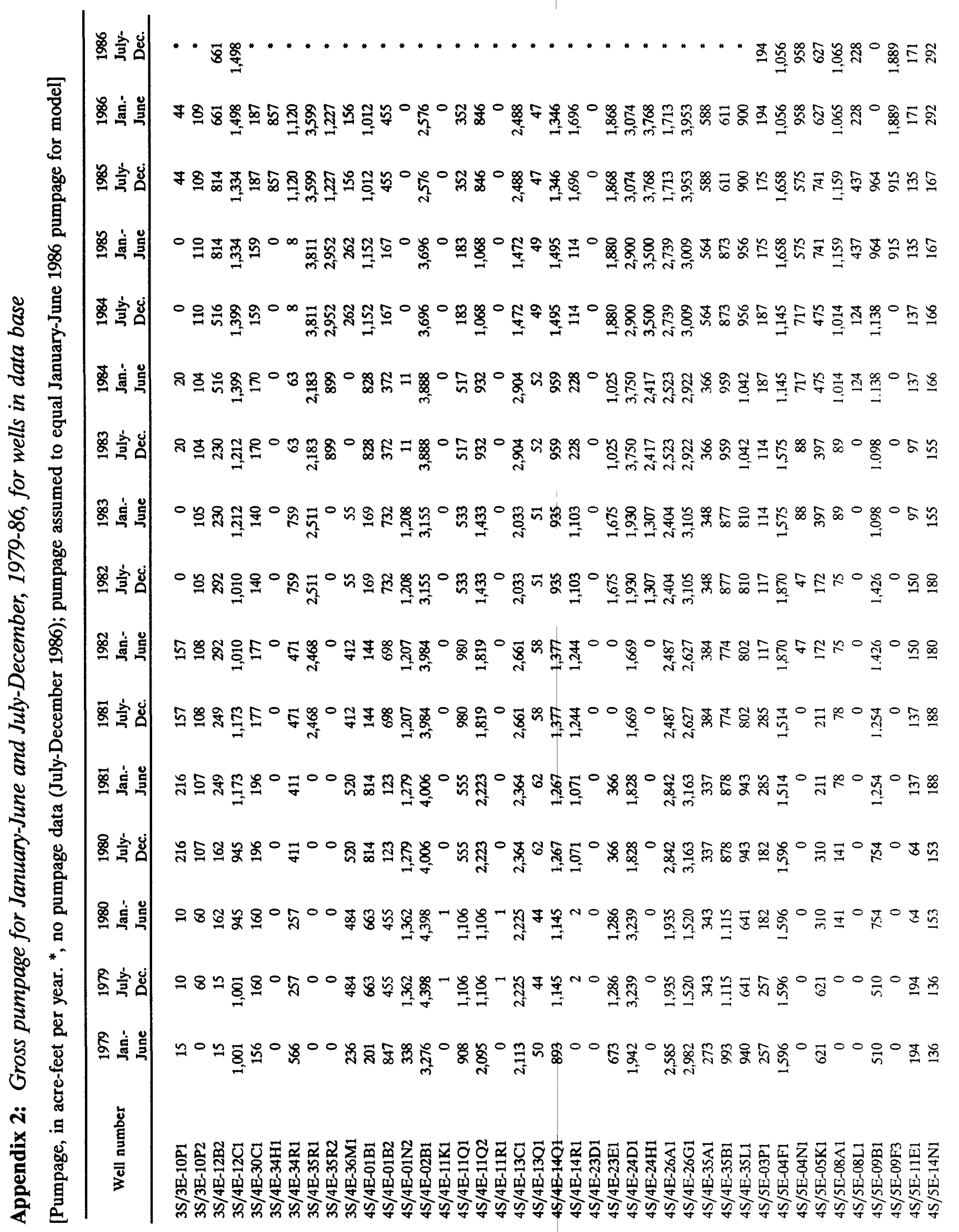




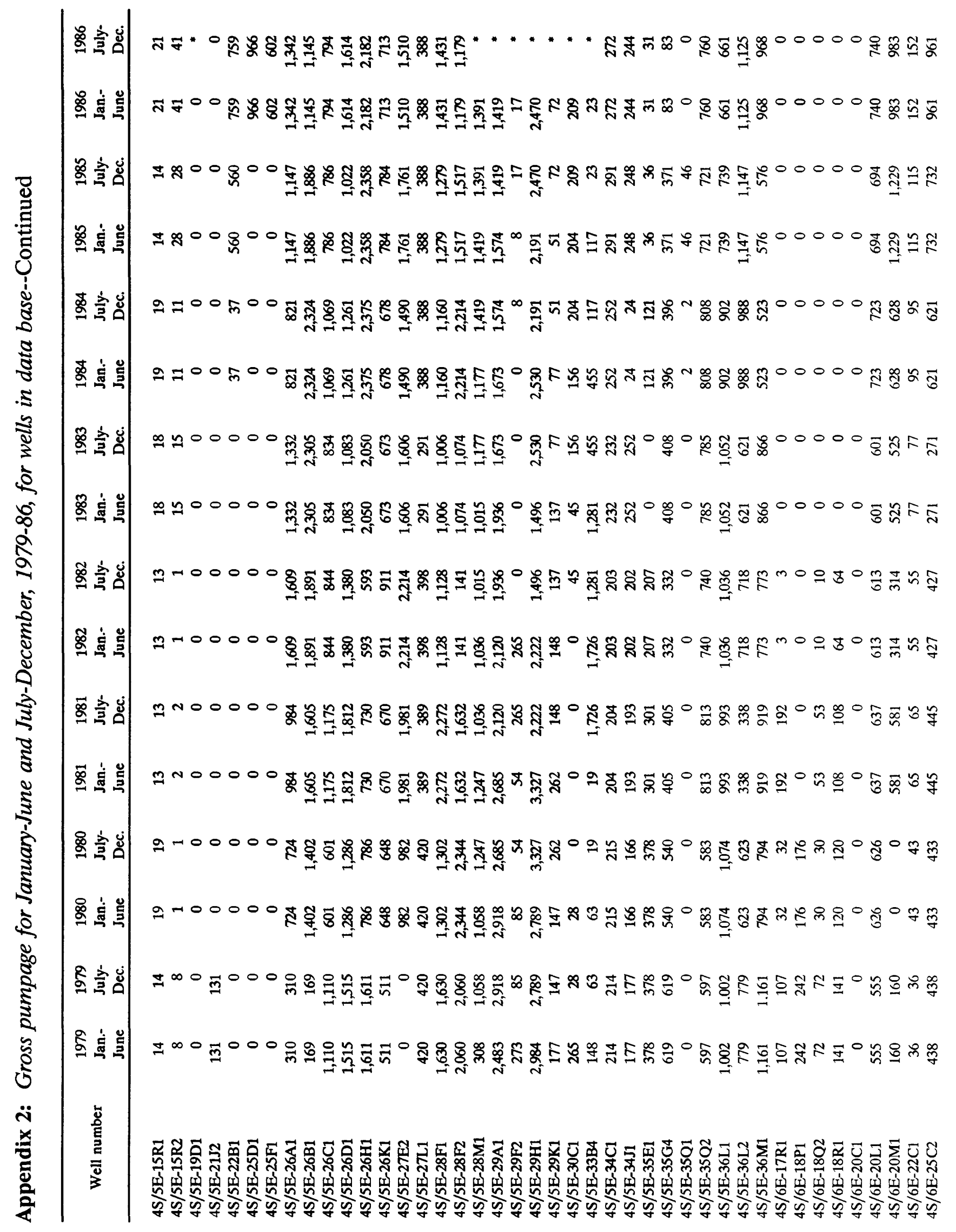




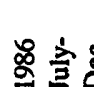
\&

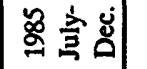

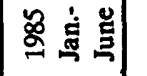

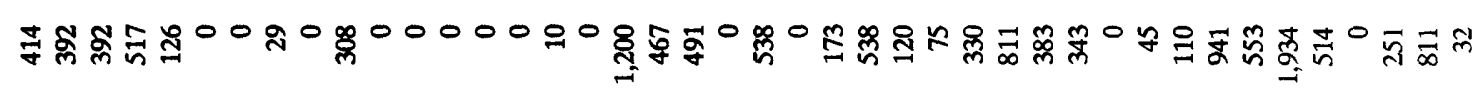

ล̊

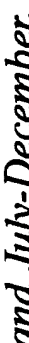

$\stackrel{\frac{1}{3}}{1}$

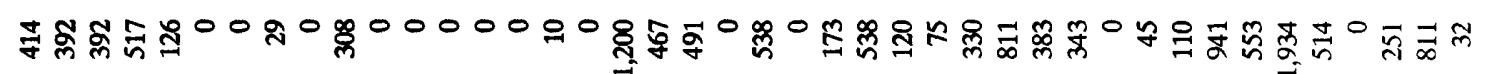

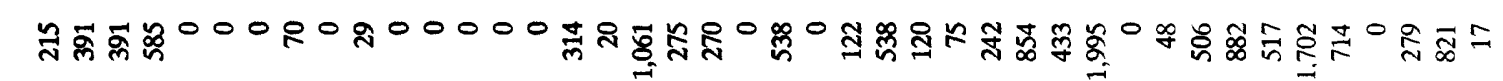

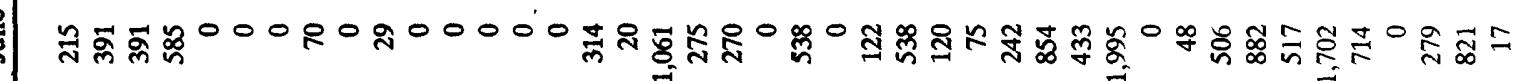

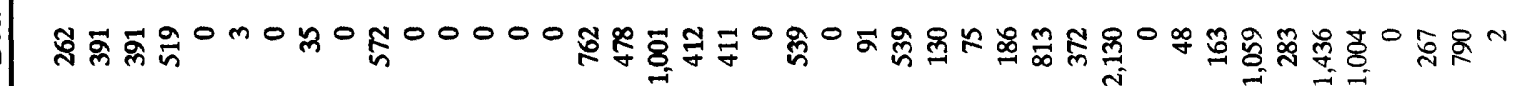

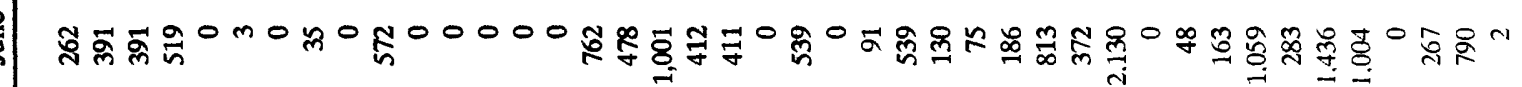
$R$ ROO

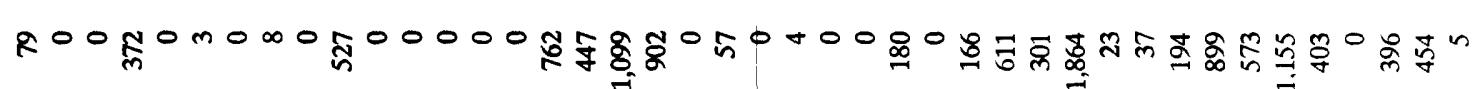
乐 융

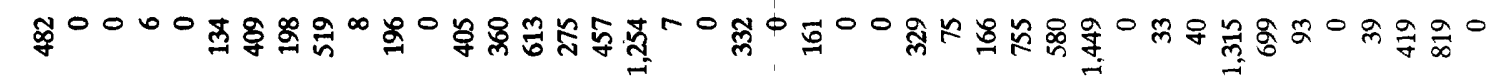

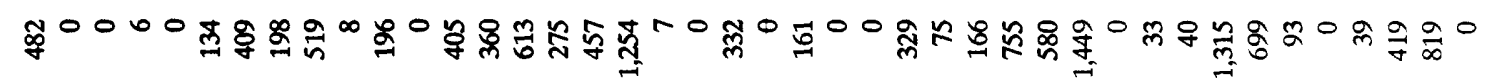
采0000反

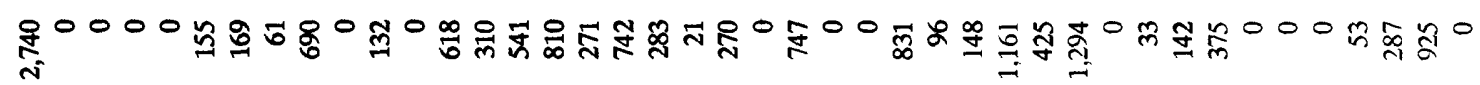

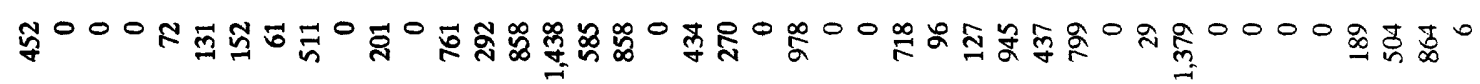

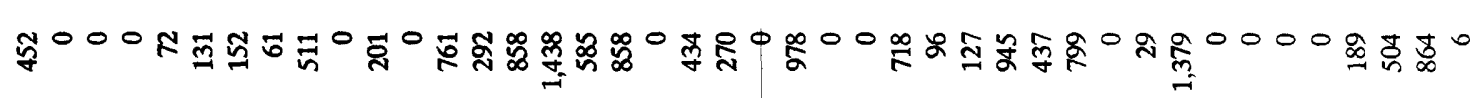

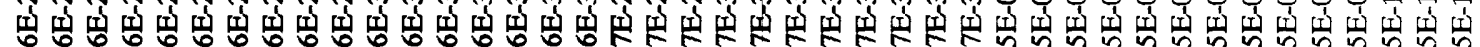

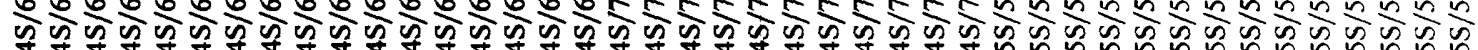




\begin{tabular}{|c|c|}
\hline & 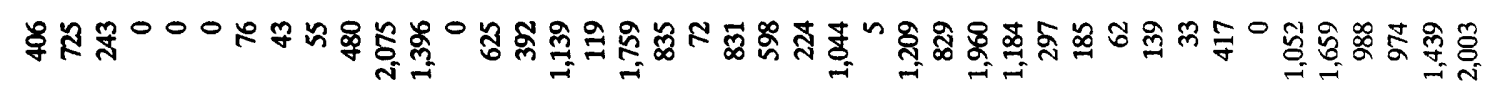 \\
\hline & 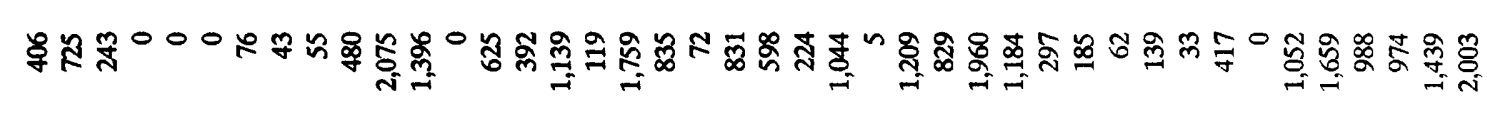 \\
\hline & 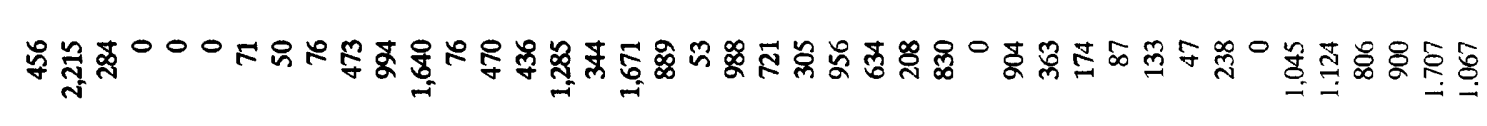 \\
\hline & 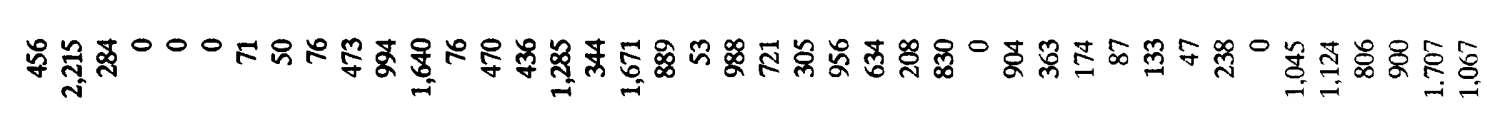 \\
\hline & 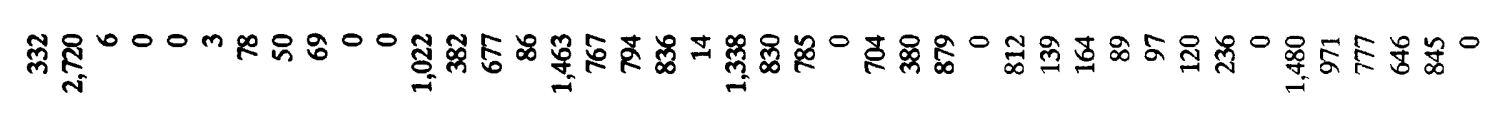 \\
\hline & 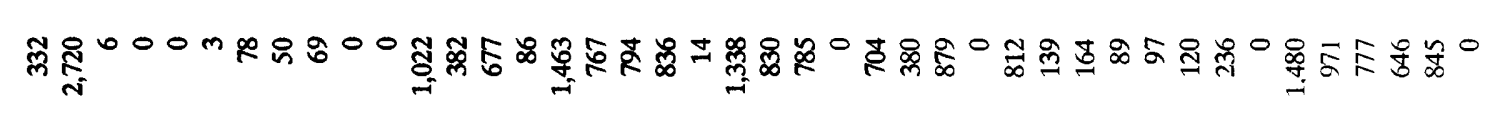 \\
\hline & 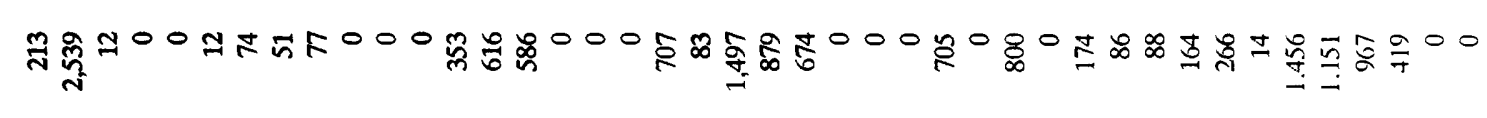 \\
\hline & 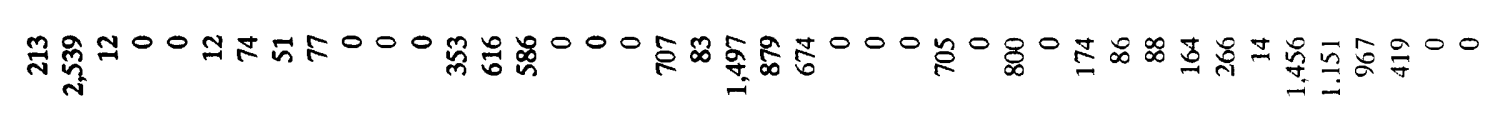 \\
\hline & 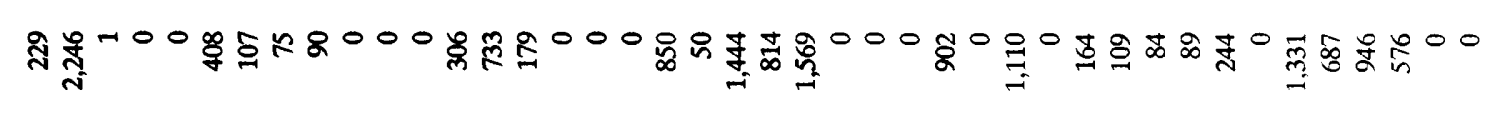 \\
\hline & 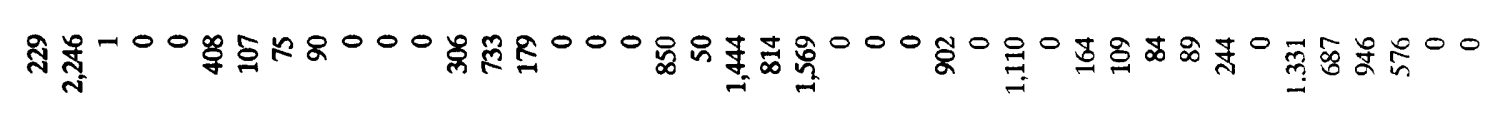 \\
\hline & 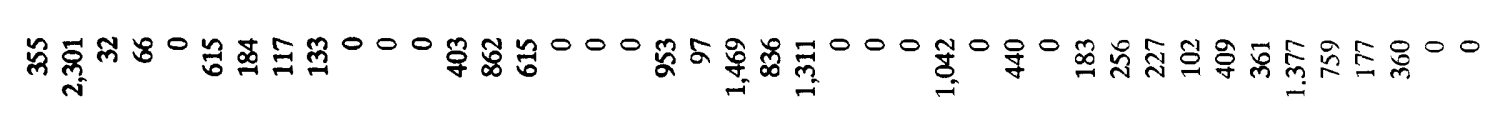 \\
\hline & 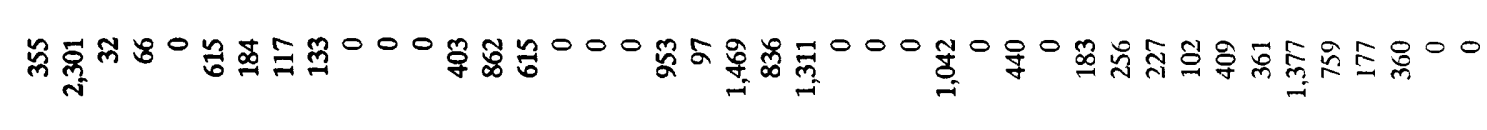 \\
\hline & 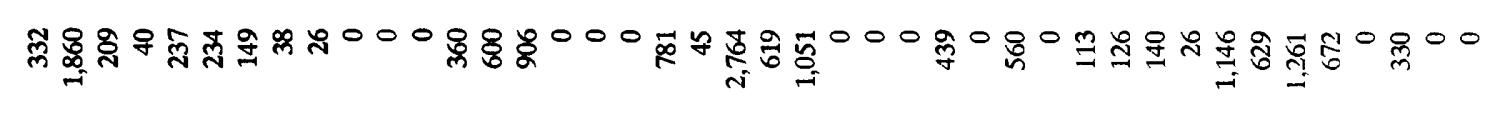 \\
\hline & 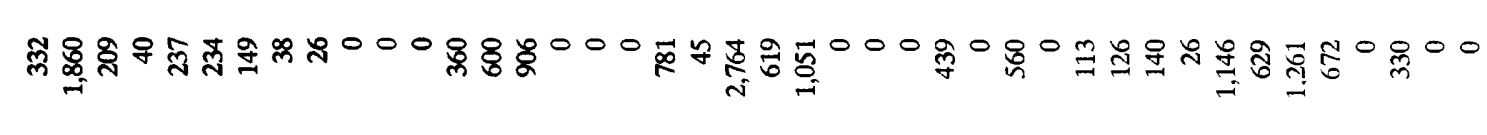 \\
\hline & 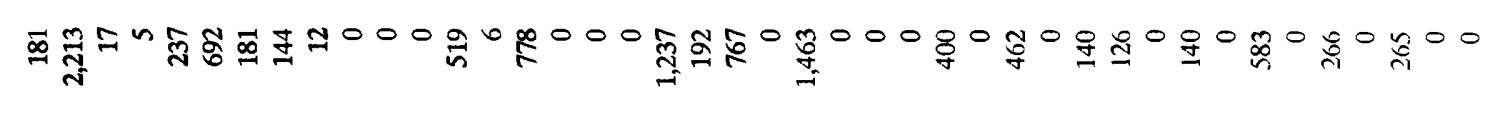 \\
\hline & 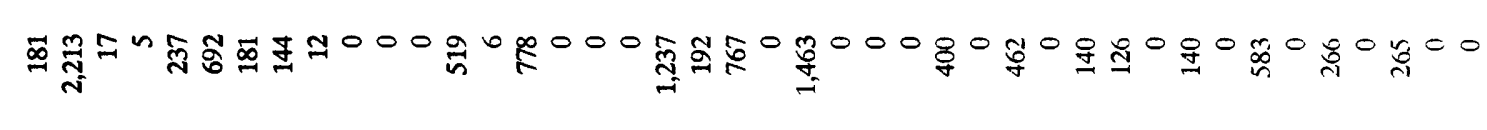 \\
\hline & 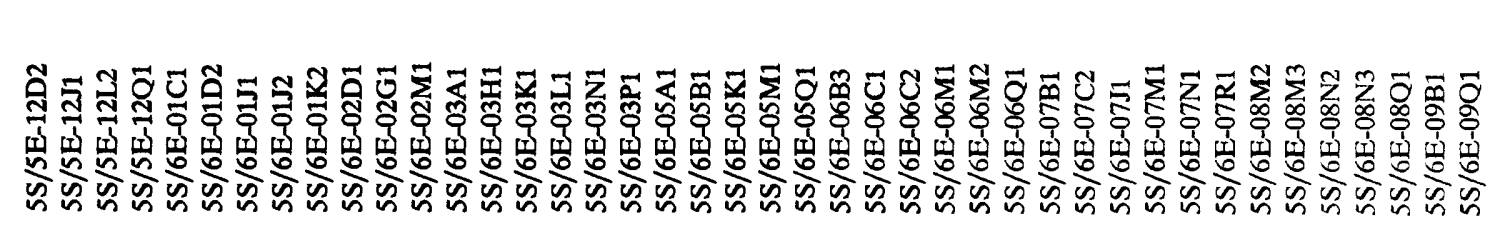 \\
\hline
\end{tabular}




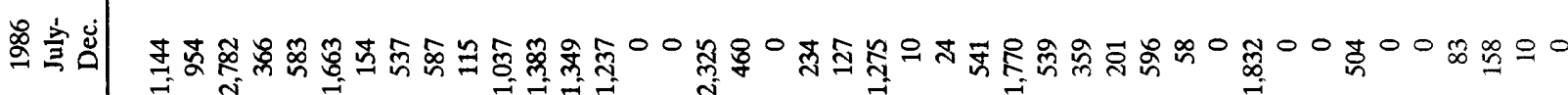

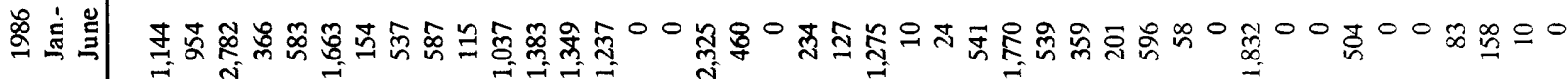

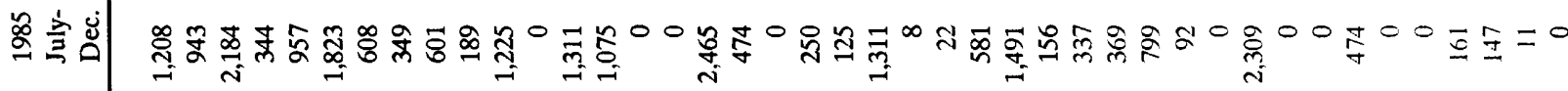

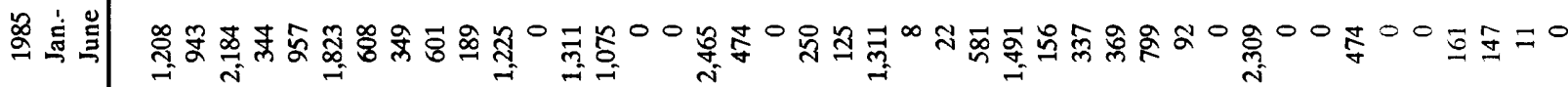

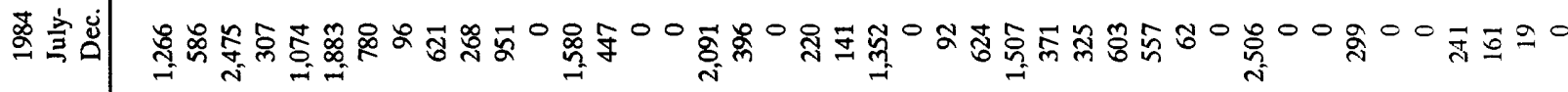

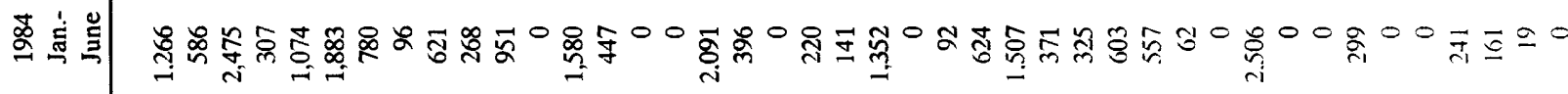

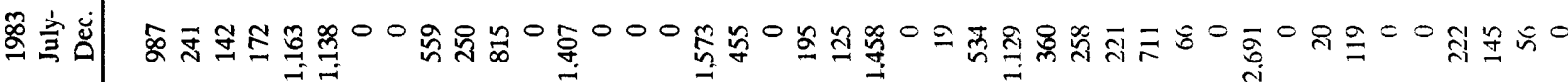

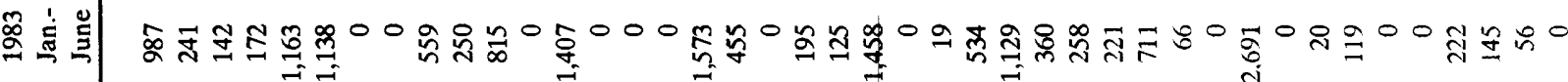

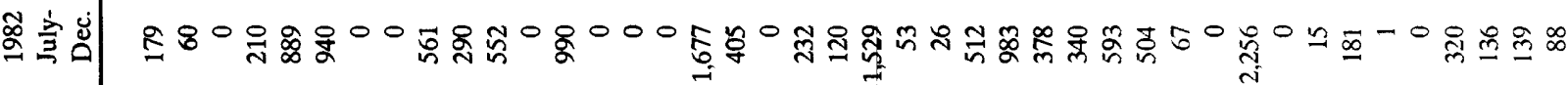

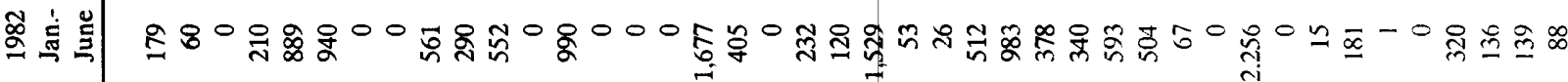

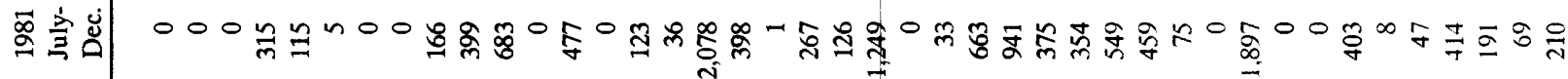

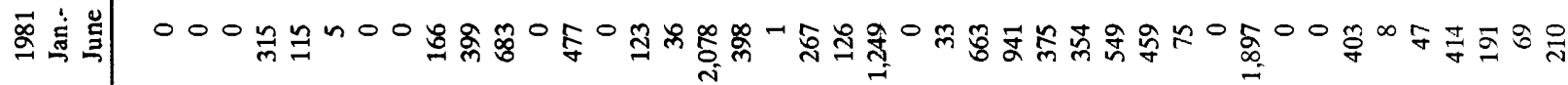

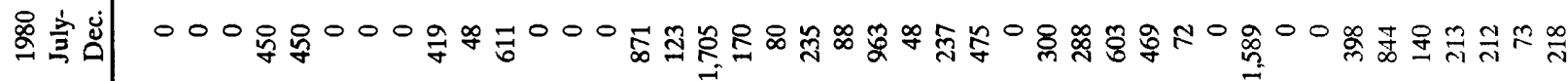

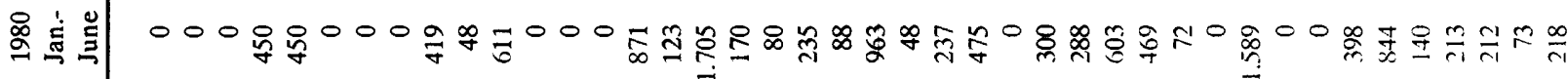

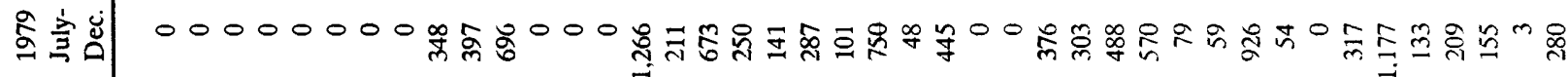

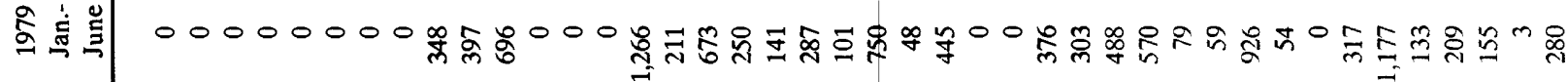

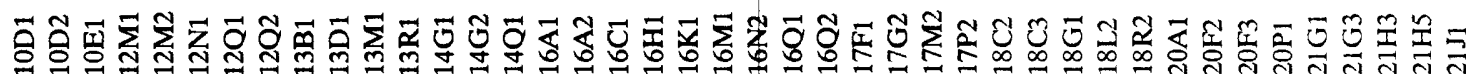

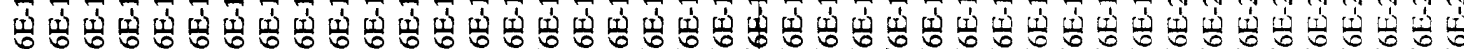

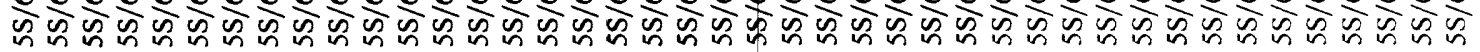




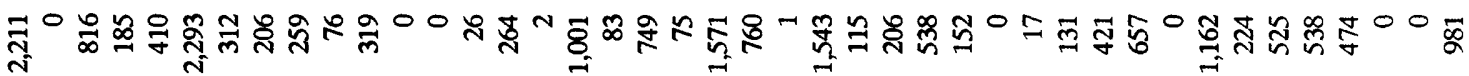

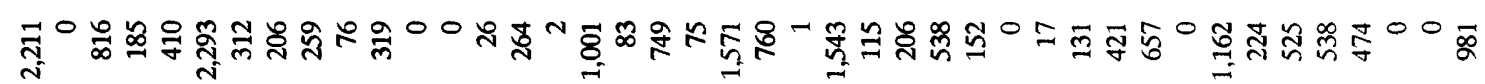

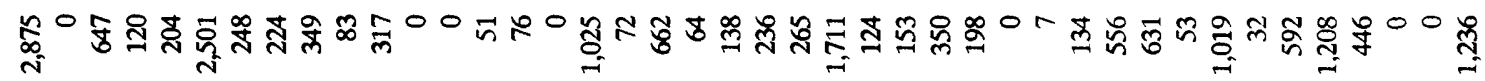

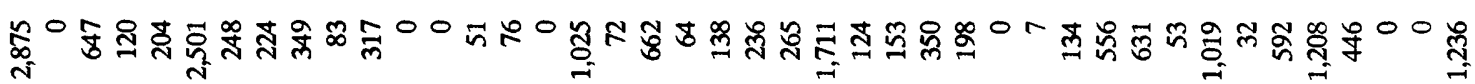

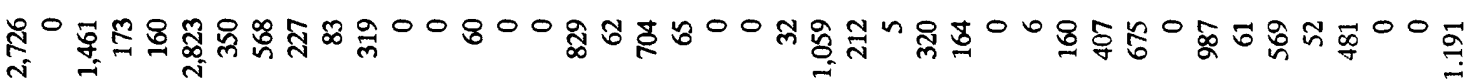

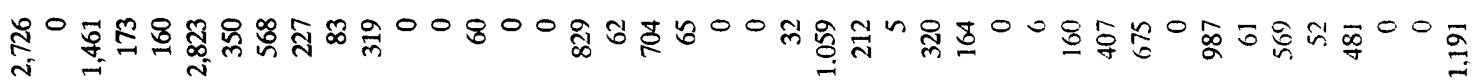

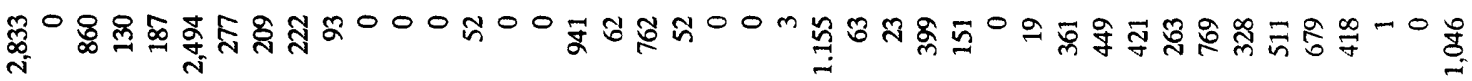

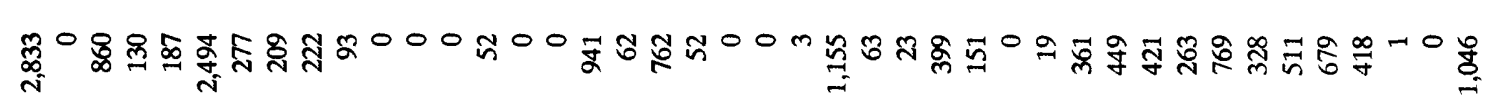

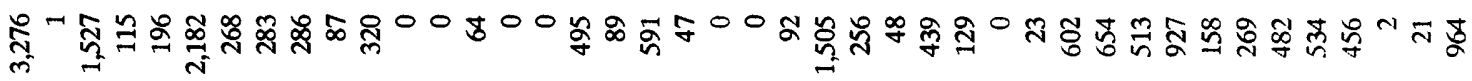

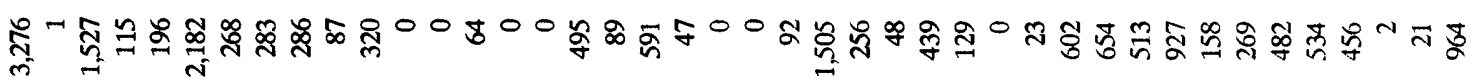

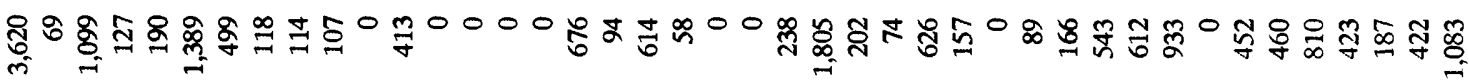

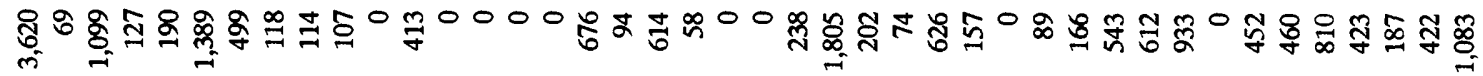

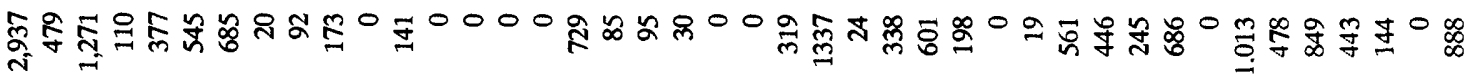

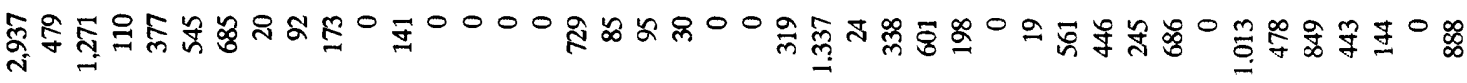

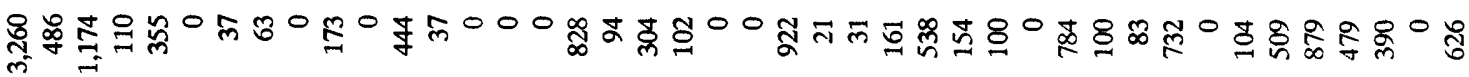

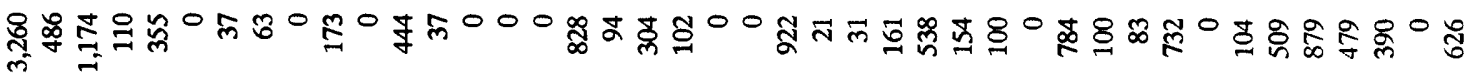

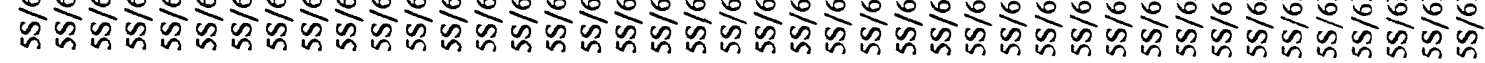




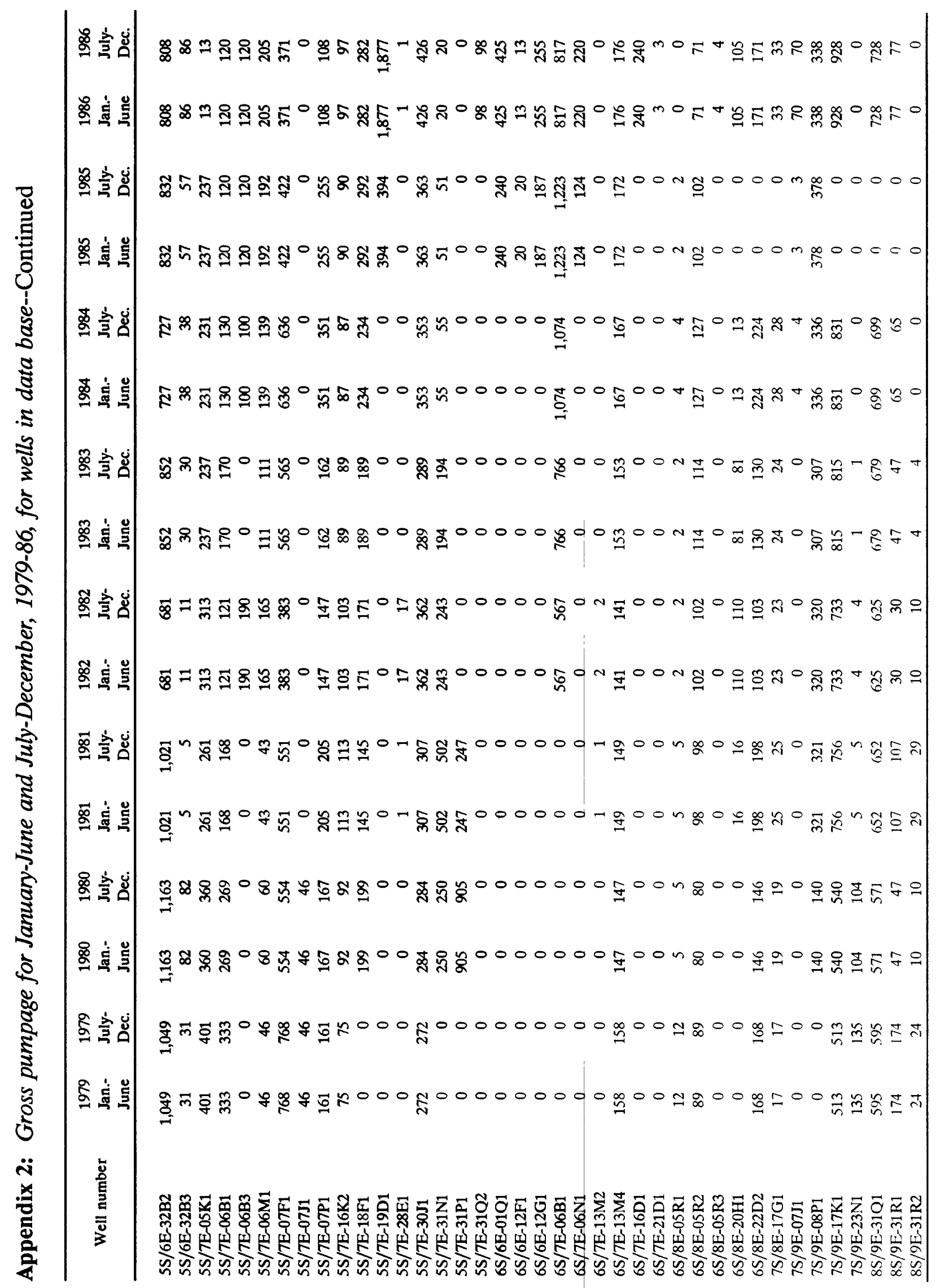


Appendix 3: Ground-water levels, autumn 1978

[Altitude of water level in feet above or below (-) sea level]

\begin{tabular}{|c|c|c|c|c|c|c|c|}
\hline $\begin{array}{c}\text { Well } \\
\text { number }\end{array}$ & $\begin{array}{c}\text { Perforated } \\
\text { interval } \\
\text { (depth, in feet } \\
\text { below land surface) }\end{array}$ & Date & $\begin{array}{c}\text { Altitude } \\
\text { of water } \\
\text { level }\end{array}$ & $\begin{array}{c}\text { Well } \\
\text { number }\end{array}$ & $\begin{array}{c}\text { Perforated } \\
\text { interval } \\
\text { (depth, in feet } \\
\text { below land surface) }\end{array}$ & Date & $\begin{array}{c}\text { Altitude } \\
\text { of water } \\
\text { level }\end{array}$ \\
\hline $2 \mathrm{~S} / 3 \mathrm{E}-25 \mathrm{~K} 1$ & -- & $09 / 78$ & 1,994 & 4S/4E-23E1 & $240-472$ & $10 / 78$ & 175 \\
\hline $2 \mathrm{~S} / 4 \mathrm{E}-34 \mathrm{~A} 1$ & $380-610$ & $09 / 78$ & 760 & $4 \mathrm{~S} / 4 \mathrm{E}-26 \mathrm{~A} 1$ & $450-780$ & $10 / 78$ & 148 \\
\hline 2S/4E-35Q1 & $185-380$ & $09 / 78$ & 748 & $4 \mathrm{~S} / 4 \mathrm{E}-35 \mathrm{~K} 1$ & $435-600$ & $09 / 78$ & 229 \\
\hline 2S/5E-31L1 & $220-285$ & $09 / 78$ & 748 & $4 \mathrm{~S} / 5 \mathrm{E}-04 \mathrm{~F} 1$ & $276-576$ & $10 / 78$ & 156 \\
\hline $2 \mathrm{~S} / 5 \mathrm{E}-32 \mathrm{E} 6$ & $110-180$ & $09 / 78$ & 1,107 & $4 \mathrm{~S} / 5 \mathrm{E}-05 \mathrm{~K} 1$ & $408-748$ & $10 / 78$ & 163 \\
\hline $2 \mathrm{~S} / 5 \mathrm{E}-33 \mathrm{E} 5$ & $261-281$ & $09 / 78$ & 1,071 & 4S/5E-09B1 & $398-806$ & $10 / 78$ & 160 \\
\hline $3 \mathrm{~S} / 2 \mathrm{E}-23 \mathrm{~B} 1$ & -- & $09 / 78$ & 1,220 & $4 \mathrm{~S} / 5 \mathrm{E}-09 \mathrm{~F} 1$ & $227-616$ & $10 / 78$ & 149 \\
\hline $3 \mathrm{~S} / 3 \mathrm{E}-08 \mathrm{M} 1$ & $225-553$ & $09 / 78$ & 1,128 & $4 \mathrm{~S} / 5 \mathrm{E}-11 \mathrm{E} 1$ & $172-607$ & $10 / 78$ & 122 \\
\hline 3S/3E-10P1 & $476-776$ & $10 / 78$ & 648 & $4 \mathrm{~S} / 5 \mathrm{E}-19 \mathrm{D} 1$ & $240-440$ & $10 / 78$ & 161 \\
\hline $3 S / 3 E-10 P 2$ & $306-906$ & $09 / 78$ & 656 & $4 \mathrm{~S} / 5 \mathrm{E}-21 \mathrm{~A} 1$ & -- & $10 / 78$ & 127 \\
\hline $3 \mathrm{~S} / 4 \mathrm{E}-12 \mathrm{~B} 1$ & $180-220$ & $10 / 78$ & 743 & $4 \mathrm{~S} / 5 \mathrm{E}-21 \mathrm{~J} 2$ & $440-470$ & $10 / 78$ & 128 \\
\hline $3 S / 4 E-12 B 2$ & $270-500$ & $10 / 78$ & 747 & & $680-1,000$ & & \\
\hline $3 \mathrm{~S} / 4 \mathrm{E}-12 \mathrm{C} 1$ & $200-480$ & $10 / 78$ & 743 & $4 \mathrm{~S} / 5 \mathrm{E}-22 \mathrm{~A} 1$ & -- & $09 / 78$ & 123 \\
\hline \multirow[t]{2}{*}{$3 \mathrm{~S} / 4 \mathrm{E}-12 \mathrm{H} 1$} & $122-147$ & $10 / 78$ & 747 & $4 \mathrm{~S} / 5 \mathrm{E}-27 \mathrm{E} 1$ & $344-392$ & $10 / 78$ & 118 \\
\hline & $172-192$ & & & $4 \mathrm{~S} / 5 \mathrm{E}-29 \mathrm{~K} 1$ & -- & $09 / 78$ & 134 \\
\hline $3 \mathrm{~S} / 4 \mathrm{E}-13 \mathrm{~N} 1$ & $216-254$ & $09 / 78$ & 485 & $4 \mathrm{~S} / 5 \mathrm{E}-30 \mathrm{C} 1$ & $300-440$ & $09 / 78$ & 141 \\
\hline $3 S / 4 E-17 K 1$ & $340-375$ & $09 / 78$ & 564 & $4 \mathrm{~S} / 5 \mathrm{E}-33 \mathrm{~B} 1$ & $268-288$ & $10 / 78$ & 118 \\
\hline 3S/4E-20D1 & $560-580$ & $10 / 78$ & 372 & $4 \mathrm{~S} / 5 \mathrm{E}-35 \mathrm{D} 2$ & $215-407$ & $09 / 78$ & 94 \\
\hline \multirow[t]{2}{*}{$3 \mathrm{~S} / 4 \mathrm{E}-22 \mathrm{~A} 1$} & $180-185$ & $09 / 78$ & 546 & $4 \mathrm{~S} / 5 \mathrm{E}-35 \mathrm{G} 3$ & -- & $10 / 78$ & 84 \\
\hline & $218-230$ & & & $4 \mathrm{~S} / 5 \mathrm{E}-35 \mathrm{G} 4$ & $600-1,000$ & $10 / 78$ & 83 \\
\hline $3 S / 4 E-29 F 1$ & $550-570$ & $10 / 78$ & 375 & $4 \mathrm{~S} / 5 \mathrm{E}-36 \mathrm{D} 1$ & $470-492$ & $09 / 78$ & 88 \\
\hline 3S/4E-29R1 & $431-551$ & $10 / 78$ & 276 & & $658-678$ & & \\
\hline $3 \mathrm{~S} / 4 \mathrm{E}-30 \mathrm{C} 1$ & $530-726$ & $10 / 78$ & 392 & $4 \mathrm{~S} / 5 \mathrm{E}-36 \mathrm{M} 1$ & $315-627$ & $10 / 78$ & 86 \\
\hline $3 \mathrm{~S} / 4 \mathrm{E}-34 \mathrm{R} 1$ & $506-830$ & $10 / 78$ & 196 & $4 \mathrm{~S} / 6 \mathrm{E}-08 \mathrm{~L} 1$ & -- & $09 / 78$ & 83 \\
\hline $3 S / 5 E-03 L 1$ & $225-370$ & $09 / 78$ & 945 & $4 \mathrm{~S} / 6 \mathrm{E}-17 \mathrm{R} 1$ & $166-817$ & $10 / 78$ & 75 \\
\hline 3S/5E-03R1 & $145-240$ & $09 / 78$ & 905 & $4 \mathrm{~S} / 6 \mathrm{E}-18 \mathrm{P} 1$ & $216-454$ & $10 / 78$ & 84 \\
\hline $3 \mathrm{~S} / 5 \mathrm{E}-04 \mathrm{H} 1$ & $244-355$ & $09 / 78$ & 914 & $4 \mathrm{~S} / 6 \mathrm{E}-18 \mathrm{Q} 2$ & $125-255$ & $10 / 78$ & 86 \\
\hline 3S/5E-09C1 & $262-334$ & $09 / 78$ & 756 & $4 \mathrm{~S} / 6 \mathrm{E}-18 \mathrm{R} 1$ & $479-518$ & $10 / 78$ & 83 \\
\hline $3 S / 5 E-10 L 2$ & $190-260$ & $10 / 78$ & 750 & $4 \mathrm{~S} / 6 \mathrm{E}-19 \mathrm{~J} 2$ & $171-500$ & $09 / 78$ & 85 \\
\hline $3 S / 5 E-10 R 1$ & $177-207$ & $09 / 78$ & 861 & $4 \mathrm{~S} / 6 \mathrm{E}-20 \mathrm{M} 1$ & $+60-960$ & $10 / 78$ & 76 \\
\hline $3 \mathrm{~S} / 5 \mathrm{E}-11 \mathrm{Q} 1$ & $170-236$ & $09 / 78$ & 882 & $4 \mathrm{~S} / 6 \mathrm{E}-22 \mathrm{C} 1$ & $208-211$ & $10 / 78$ & 61 \\
\hline $3 \mathrm{~S} / 5 \mathrm{E}-12 \mathrm{P} 1$ & $304-379$ & $09 / 78$ & 859 & & $272-304$ & & \\
\hline $3 \mathrm{~S} / 5 \mathrm{E}-17 \mathrm{~J} 1$ & $182-405$ & $10 / 78$ & 740 & $4 \mathrm{~S} / 6 \mathrm{E}-22 \mathrm{C} 2$ & $189-213$ & $10 / 78$ & 67 \\
\hline 3S/5E-19B1 & $51-83$ & $09 / 78$ & 692 & & $233-305$ & & \\
\hline $3 S / 5 E-30 G 1$ & $344-644$ & $09 / 78$ & 387 & $4 \mathrm{~S} / 6 \mathrm{E}-22 \mathrm{~J} 1$ & -- & $09 / 78$ & 69 \\
\hline $3 \mathrm{~S} / 6 \mathrm{E}-25 \mathrm{Q} 1$ & $371-511$ & $09 / 78$ & 728 & $4 \mathrm{~S} / 6 \mathrm{E}-22 \mathrm{~K} 1$ & $150-495$ & $09 / 78$ & 80 \\
\hline $3 \mathrm{~S} / 6 \mathrm{E}-26 \mathrm{P} 1$ & 204-310 & $09 / 78$ & 711 & $4 \mathrm{~S} / 6 \mathrm{E}-27 \mathrm{~N} 1$ & $506-563$ & $09 / 78$ & 46 \\
\hline $3 \mathrm{~S} / 6 \mathrm{E}-28 \mathrm{~A} 1$ & $250-332$ & $09 / 78$ & 745 & $4 \mathrm{~S} / 6 \mathrm{E}-28 \mathrm{~A} 2$ & $180-750$ & $10 / 78$ & 61 \\
\hline $3 S / 6 E-36 P 1$ & -- & $09 / 78$ & 689 & $4 \mathrm{~S} / 6 \mathrm{E}-28 \mathrm{~J} 2$ & -- & $09 / 78$ & 57 \\
\hline 4S/4E-01B3 & $400-700$ & $10 / 78$ & 160 & $4 \mathrm{~S} / 6 \mathrm{E}-29 \mathrm{~A} 1$ & $178-844$ & $09 / 78$ & 70 \\
\hline 4S/4E-01N2 & $525-800$ & $09 / 78$ & 175 & $4 \mathrm{~S} / 6 \mathrm{E}-34 \mathrm{D} 1$ & -- & $10 / 78$ & 46 \\
\hline 4S/4E-11R1 & $526-700$ & $10 / 78$ & 166 & $4 \mathrm{~S} / 6 \mathrm{E}-34 \mathrm{~F} 1$ & -- & $10 / 78$ & 81 \\
\hline $4 \mathrm{~S} / 4 \mathrm{E}-13 \mathrm{H} 1$ & $400-500$ & $09 / 78$ & 163 & $4 \mathrm{~S} / 6 \mathrm{E}-34 \mathrm{Q} 1$ & $171-602$ & $09 / 78$ & 92 \\
\hline \multirow[t]{2}{*}{$4 \mathrm{~S} / 4 \mathrm{E}-13 \mathrm{P} 1$} & $345-365$ & $09 / 78$ & 170 & 4S/7E-14E1 & $382-520$ & $09 / 78$ & 726 \\
\hline & $398-408$ & & & 4S/7E-30M1 & -. & $11 / 78$ & 30 \\
\hline \multirow[t]{2}{*}{ 4S/4E-15J1 } & $164-344$ & $09 / 78$ & 167 & 4S/7E-31Q3 & $236-356$ & $09 / 78$ & 10 \\
\hline & $415-435$ & & & & $590-710$ & & \\
\hline
\end{tabular}




\begin{tabular}{|c|c|c|c|c|c|c|c|}
\hline $\begin{array}{l}\text { Well } \\
\text { number }\end{array}$ & $\begin{array}{c}\text { Perforated } \\
\text { interval } \\
\text { (depth, in feet } \\
\text { below land surface) }\end{array}$ & Date & $\begin{array}{c}\text { Altitude } \\
\text { of water } \\
\text { level }\end{array}$ & $\begin{array}{l}\text { Well } \\
\text { number }\end{array}$ & $\begin{array}{c}\text { Perforaled } \\
\text { interval } \\
\text { (depth, in fect } \\
\text { below land surface) }\end{array}$ & Date & $\begin{array}{l}\text { Altitude } \\
\text { ol waler } \\
\text { level }\end{array}$ \\
\hline $4 \mathrm{~S} / 7 \mathrm{E}-32 \mathrm{~N} 2$ & $195-462$ & $10 / 78$ & 2 & $5 \mathrm{~S} / 6 \mathrm{E}-22 \mathrm{P} 1$ & $254-292$ & $11 / 78$ & 34 \\
\hline $4 \mathrm{~S} / 7 \mathrm{E}-33 \mathrm{~N} 1$ & $100-412$ & $09 / 78$ & 4 & & $305-370$ & & \\
\hline 5S/5E-01D2 & -- & $09 / 78$ & 82 & $5 \mathrm{~S} / 6 \mathrm{E}-22 \mathrm{P} 2$ & $264-370$ & $11 / 78$ & 47 \\
\hline 5S/5E-01L5 & $500-740$ & $10 / 78$ & 72 & $5 \mathrm{~S} / 6 \mathrm{E}-23 \mathrm{~L} 3$ & $255-312$ & $11 / 78$ & 23 \\
\hline $5 \mathrm{~S} / 5 \mathrm{E}-01 \mathrm{P} 1$ & $108-192$ & $10 / 78$ & 75 & 5S/6E-23M1 & $384-480$ & $11 / 78$ & 32 \\
\hline $5 \mathrm{~S} / 5 \mathrm{E}-02 \mathrm{~F} 2$ & $250-495$ & $10 / 78$ & 73 & & $576-776$ & & \\
\hline \multirow[t]{2}{*}{$5 \mathrm{~S} / 5 \mathrm{E}-02 \mathrm{~L} 1$} & $130-140$ & $09 / 78$ & 82 & 5S/6E-24G1 & $480-680$ & $11 / 78$ & 6 \\
\hline & $200-220$ & & & & $810-930$ & & \\
\hline $5 \mathrm{~S} / 5 \mathrm{E}-02 \mathrm{Q} 1$ & $220-450$ & $09 / 78$ & 71 & $5 \mathrm{~S} / 6 \mathrm{E}-25 \mathrm{~A} 1$ & $40(0-6.56$ & (0) $/ 78$ & 5 \\
\hline \multirow[t]{2}{*}{$5 \mathrm{~S} / 5 \mathrm{E}-03 \mathrm{~A} 1$} & $110-130$ & $09 / 78$ & 88 & $5 S / 6 E-27 C 1$ & $138-292$ & $10 / 78$ & 46 \\
\hline & $238-320$ & & & $5 S / 6 E-27 C 2$ & -- & $11 / 78$ & 46 \\
\hline $5 \mathrm{~S} / 5 \mathrm{E}-11 \mathrm{~A} 1$ & -- & $10 / 78$ & 56 & $5 \mathrm{~S} / 6 \mathrm{E}-28 \mathrm{C} 1$ & $204-252$ & $11 / 78$ & 47 \\
\hline $5 \mathrm{~S} / 5 \mathrm{E}-12 \mathrm{C} 1$ & $162-503$ & $10 / 78$ & 78 & $5 \mathrm{~S} / 6 \mathrm{E}-28 \mathrm{C} 2$ & $410-680$ & $11 / 78$ & 48 \\
\hline $5 \mathrm{~S} / 5 \mathrm{E}-12 \mathrm{C} 2$ & -- & $09 / 78$ & 64 & $5 \mathrm{~S} / 6 \mathrm{E}-28 \mathrm{E} 1$ & $247-346$ & $09 / 78$ & 45 \\
\hline $5 \mathrm{~S} / 5 \mathrm{E}-12 \mathrm{D} 1$ & $125-360$ & $09 / 78$ & 73 & $5 \mathrm{~S} / 6 \mathrm{E}-29 \mathrm{~B} 1$ & $351-615$ & $09 / 78$ & 47 \\
\hline $5 \mathrm{~S} / 5 \mathrm{E}-12 \mathrm{H} 2$ & $432-590$ & $10 / 78$ & 57 & $5 S / 6 E-29 C 1$ & $243-531$ & $10 / 78$ & 29 \\
\hline $5 \mathrm{~S} / 5 \mathrm{E}-12 \mathrm{~J} 1$ & $400-880$ & $10 / 78$ & 46 & $5 S / 6 E-29 C 2$ & $530-670$ & $10 / 78$ & 38 \\
\hline $5 \mathrm{~S} / 5 \mathrm{E}-12 \mathrm{~L} 2$ & $208-461$ & $10 / 78$ & 70 & $5 \mathrm{~S} / 6 \mathrm{E}-29 \mathrm{M} 1$ & $457-723$ & $11 / 78$ & 49 \\
\hline $5 \mathrm{~S} / 5 \mathrm{E}-12 \mathrm{Q} 1$ & -- & $10 / 78$ & 68 & 5S/6E-29P1 & $500-700$ & $10 / 78$ & 43 \\
\hline $5 \mathrm{~S} / 6 \mathrm{E}-02 \mathrm{~A} 1$ & $200-618$ & $10 / 78$ & 38 & $5 S / 6 E-32 G 1$ & $459-7.36$ & $10 / 78$ & 120 \\
\hline $5 \mathrm{~S} / 6 \mathrm{E}-02 \mathrm{~A} 2$ & $450-650$ & $10 / 78$ & 21 & $5 S / 7 E-04 A 1$ & $147-367$ & $09 / 78$ & -3 \\
\hline $5 \mathrm{~S} / 6 \mathrm{E}-06 \mathrm{Q} 1$ & $668-1,202$ & $11 / 78$ & 61 & $5 S / 7 E-04 D 1$ & $100-370$ & $09 / 78$ & 0 \\
\hline 5S/6E-07J1 & $460-560$ & $10 / 78$ & 59 & 5S/7E-04M1 & $192-678$ & $09 / 78$ & -3 \\
\hline $5 \mathrm{~S} / 6 \mathrm{E}-07 \mathrm{Q} 2$ & $300-500$ & $10 / 78$ & 55 & $5 \mathrm{~S} / 7 \mathrm{E}-05 \mathrm{~K} 1$ & -- & $10 / 78$ & $-9)$ \\
\hline $5 \mathrm{~S} / 6 \mathrm{E}-07 \mathrm{Q} 3$ & $380-500$ & $10 / 78$ & 57 & 5S/7E-06B1 & $168-180$ & $10 / 78$ & 8 \\
\hline $5 \mathrm{~S} / 6 \mathrm{E}-08 \mathrm{~N} 2$ & $450-780$ & $09 / 78$ & 65 & & $280-303$ & & \\
\hline \multirow[t]{2}{*}{$5 \mathrm{~S} / 6 \mathrm{E}-12 \mathrm{G} 1$} & $192-272$ & $10 / 78$ & 8 & $5 \mathrm{~S} / 7 \mathrm{E}-06 \mathrm{H} 1$ & 40()$-480$ & $09 / 78$ & -2 \\
\hline & $472-604$ & & & 5S/7E-06M1 & $195-363$ & $10 / 78$ & 11 \\
\hline \multirow[t]{2}{*}{$5 \mathrm{~S} / 6 \mathrm{E}-13 \mathrm{D} 1$} & $518-566$ & $10 / 78$ & 3 & & $555-651$ & & \\
\hline & $614-878$ & & & $5 \mathrm{~S} / 7 \mathrm{E}-07 \mathrm{~F} 1$ & $147-459$ & $09 / 78$ & 14 \\
\hline \multirow[t]{2}{*}{$5 \mathrm{~S} / 6 \mathrm{E}-14 \mathrm{Q} 1$} & $440-600$ & $11 / 78$ & 22 & $5 S / 7 E-07 J 1$ & $147-363$ & $09 / 78$ & -14 \\
\hline & $706-810$ & & & 5S/7E-07P1 & $144-450$ & 10/7s & 3 \\
\hline $5 \mathrm{~S} / 6 \mathrm{E}-16 \mathrm{~A} 1$ & $360-560$ & $11 / 78$ & 40 & 5S/7E-()8(j1 & -- & $11 / 78$ & -2 \\
\hline \multirow[t]{2}{*}{$5 \mathrm{~S} / 6 \mathrm{E}-16 \mathrm{~A} 2$} & $445-530$ & $10 / 78$ & 47 & 5S/7E-08Q1 & $203-6.54$ & $09 / 78$ & -13 \\
\hline & $730-965$ & & & 5S/7E-09F1 & $130-310$ & $09 / 78$ & -16 \\
\hline $5 \mathrm{~S} / 6 \mathrm{E}-16 \mathrm{H} 1$ & $336-552$ & $10 / 78$ & 33 & $5 \mathrm{~S} / 7 \mathrm{E}-10 \mathrm{E} 1$ & $70-360$ & $09 / 78$ & -18 \\
\hline $5 \mathrm{~S} / 6 \mathrm{E}-16 \mathrm{M} 1$ & $350-500$ & $09 / 78$ & 42 & $5 \mathrm{~S} / 7 \mathrm{E}-11 \mathrm{C} 1$ & $323-43.3$ & $09 / 78$ & -12 \\
\hline $5 \mathrm{~S} / 6 \mathrm{E}-18 \mathrm{~L} 2$ & $317-509$ & $11 / 78$ & 44 & $5 \mathrm{~S} / 7 \mathrm{E}-12 \mathrm{P} 1$ & $280-400$ & $09 / 78$ & -28 \\
\hline $5 \mathrm{~S} / 6 \mathrm{E}-18 \mathrm{R} 1$ & $296-450$ & $10 / 78$ & 43 & 5S/7E-13D1 & $580-583$ & $09 / 78$ & -30 \\
\hline $5 \mathrm{~S} / 6 \mathrm{E}-18 \mathrm{R} 2$ & $425-800$ & $10 / 78$ & 41 & & $980-1,020$ & & \\
\hline $5 \mathrm{~S} / 6 \mathrm{E}-20 \mathrm{P} 1$ & $300-588$ & $11 / 78$ & 37 & $5 \mathrm{~S} / 7 \mathrm{E}-14 \mathrm{~J} 2$ & $232-350$ & $09 / 78$ & -27 \\
\hline $5 \mathrm{~S} / 6 \mathrm{E}-21 \mathrm{~L} 1$ & $250-578$ & $10 / 78$ & 45 & $5 \mathrm{~S} / 7 \mathrm{E}-14 \mathrm{~K} 1$ & $264-411$ & $09 / 78$ & -29 \\
\hline $5 S / 6 E-21 N 2$ & $400-820$ & $11 / 78$ & 45 & $5 \mathrm{~S} / 7 \mathrm{E}-15 \mathrm{Q} 1$ & $264-464$ & $09 / 78$ & -24 \\
\hline $5 S / 6 E-21 Q 3$ & $350-700$ & $11 / 78$ & 46 & $5 \mathrm{~S} / 7 \mathrm{E}-16 \mathrm{C} 1$ & $147-355$ & $10 / 78$ & -11 \\
\hline \multirow[t]{4}{*}{$5 \mathrm{~S} / 6 \mathrm{E}-22 \mathrm{~B} 1$} & $350-390$ & $9 / 78$ & 26 & $5 \mathrm{~S} / 7 \mathrm{E}-16 \mathrm{~K} 2$ & $200-415$ & $10 / 78$ & -18 \\
\hline & $416-452$ & & & 5S/7E-18D1 & $160-200$ & $09 / 78$ & .9 \\
\hline & $468-502$ & & & $5 \mathrm{~S} / 7 \mathrm{E}-18 \mathrm{M} 2$ & $168-204$ & $10 / 78$ & 4 \\
\hline & & & & $5 \mathrm{~S} / 7 \mathrm{E}-22 \mathrm{H} 2$ & $506-1,100$ & $10 / 78$ & -42 \\
\hline
\end{tabular}




\begin{tabular}{|c|c|c|c|c|c|c|c|}
\hline $\begin{array}{c}\text { Well } \\
\text { number }\end{array}$ & $\begin{array}{c}\text { Perforated } \\
\text { interval } \\
\text { (depth, in feet } \\
\text { below land surface) }\end{array}$ & Date & $\begin{array}{c}\text { Altitude } \\
\text { of water } \\
\text { level }\end{array}$ & $\begin{array}{c}\text { Well } \\
\text { number }\end{array}$ & $\begin{array}{c}\text { Perlorated } \\
\text { interval } \\
\text { (depth, in feet } \\
\text { below land surface) }\end{array}$ & Datc & $\begin{array}{c}\text { Altitude } \\
\text { of water } \\
\text { level }\end{array}$ \\
\hline 5S/7E-27B1 & $236-500$ & $10 / 78$ & -29 & $6 \mathrm{~S} / 8 \mathrm{E}-05 \mathrm{P} 1$ & $216-264$ & $10 / 78$ & -83 \\
\hline $5 S / 7 E-27 L 1$ & $516-600$ & $09 / 78$ & -44 & 6S/8E-05R1 & $206-640$ & $10 / 78$ & -86 \\
\hline $5 S / 7 E-28 E 1$ & $128-198$ & $10 / 78$ & -20 & $6 \mathrm{~S} / 8 \mathrm{E}-05 \mathrm{R} 2$ & $540-750$ & $10 / 78$ & -88 \\
\hline \multirow[t]{2}{*}{$5 S / 7 E-30 C 2$} & $115-235$ & $09 / 78$ & -14 & $6 \mathrm{~S} / 8 \mathrm{E}-06 \mathrm{G} 3$ & $200-260$ & $10 / 78$ & .72 \\
\hline & $427-489$ & & & $6 \mathrm{~S} / 8 \mathrm{E}-09 \mathrm{~K} 2$ & $468-548$ & $10 / 78$ & -98 \\
\hline \multirow[t]{3}{*}{$5 S / 7 E-30 F 1$} & $77-84$ & $10 / 78$ & -17 & $6 \mathrm{~S} / 8 \mathrm{E}-09 \mathrm{Q} 4$ & $580-680$ & $10 / 78$ & -98 \\
\hline & $104-126$ & & & $6 \mathrm{~S} / 8 \mathrm{E}-10 \mathrm{~F} 1$ & $506-583$ & $10 / 78$ & -99 \\
\hline & $140-144$ & & & $6 \mathrm{~S} / 8 \mathrm{E}-17 \mathrm{R} 1$ & $470-550$ & $10 / 78$ & -98 \\
\hline $5 \mathrm{~S} / 7 \mathrm{E}-30 \mathrm{~F} 2$ & -- & $10 / 78$ & -15 & 6S/8E-19D1 & $1,196-1,413$ & $10 / 78$ & -75 \\
\hline $5 \mathrm{~S} / 7 \mathrm{E}-30 \mathrm{~J} 1$ & $500-900$ & $10 / 78$ & -22 & 6S/8E-19D2 & $450-570$ & $10 / 78$ & -94 \\
\hline 5S/7E-31N1 & $110-772$ & $11 / 78$ & -28 & 6S/8E-19R1 & $000-350$ & $10 / 78$ & -75 \\
\hline $5 S / 7 E-31 P 1$ & $110-680$ & $11 / 78$ & -19 & $6 \mathrm{~S} / 8 \mathrm{E}-22 \mathrm{D} 2$ & $500-680$ & $10 / 78$ & -118 \\
\hline $5 S / 7 E-33 D 2$ & $398-518$ & $09 / 78$ & -37 & & $900-1,100$ & & \\
\hline $5 \mathrm{~S} / 7 \mathrm{E}-33 \mathrm{~F} 2$ & $400-540$ & $09 / 78$ & -28 & $6 \mathrm{~S} / 8 \mathrm{E}-22 \mathrm{~K} 1$ & $500-1,030$ & $10 / 78$ & -134 \\
\hline 5S/7E-33M1 & $388-517$ & $10 / 78$ & -35 & $6 \mathrm{~S} / 8 \mathrm{E}-25 \mathrm{P} 1$ & $478-658$ & $10 / 78$ & -159 \\
\hline 5S/7E-36D1 & $152-756$ & $09 / 78$ & -45 & $6 \mathrm{~S} / 8 \mathrm{E}-27 \mathrm{C} 1$ & $670-1,070$ & $10 / 78$ & -121 \\
\hline $5 \mathrm{~S} / 7 \mathrm{E}-36 \mathrm{G} 1$ & $125-345$ & $09 / 78$ & -45 & $6 \mathrm{~S} / 8 \mathrm{E}-27 \mathrm{~N} 1$ & $312-438$ & $10 / 78$ & -138 \\
\hline 5S/7E-36Q1 & $147-375$ & $10 / 78$ & -49 & $6 \mathrm{~S} / 8 \mathrm{E}-32 \mathrm{R} 1$ & $0(0)-1,400)$ & $10 / 78$ & -101 \\
\hline $5 \mathrm{~S} / 8 \mathrm{E}-17 \mathrm{~N} 1$ & $278-398$ & $10 / 78$ & -32 & $6 \mathrm{~S} / 8 \mathrm{E}-34 \mathrm{C} 1$ & $447-545$ & $10 / 78$ & -1.35 \\
\hline $5 \mathrm{~S} / 8 \mathrm{E}-19 \mathrm{H} 2$ & $402-690$ & $10 / 78$ & -66 & $6 \mathrm{~S} / 8 \mathrm{E}-35 \mathrm{~J} 1$ & 514-564 & $10 / 78$ & -149 \\
\hline $5 S / 8 E-20 C 2$ & $278-438$ & $10 / 78$ & -53 & $6 \mathrm{~S} / 8 \mathrm{E}-36 \mathrm{M} 1$ & $1,540-1,880$ & $10 / 78$ & -144 \\
\hline 5S/8E-20M1 & $400-450$ & $10 / 78$ & -59 & $6 \mathrm{~S} / 9 \mathrm{E}-30 \mathrm{~A} 1$ & $220-355$ & $10 / 78$ & -104 \\
\hline 5S/8E-28M1 & $388-460$ & $10 / 78$ & -27 & $6 \mathrm{~S} / 9 \mathrm{E}-32 \mathrm{~A} 1$ & $218-598$ & $10 / 78$ & -185 \\
\hline $5 \mathrm{~S} / 8 \mathrm{E}-28 \mathrm{M} 2$ & $208-268$ & $10 / 78$ & 14 & $6 \mathrm{~S} / 9 \mathrm{E}-32 \mathrm{Q} 1$ & $244-284$ & $10 / 78$ & -175 \\
\hline 5S/8E-29G1 & $230-278$ & $10 / 78$ & 0 & $6 \mathrm{~S} / 9 \mathrm{E}-33 \mathrm{~K} 1$ & $240-402$ & $10 / 78$ & -169 \\
\hline $5 \mathrm{~S} / 8 \mathrm{E}-29 \mathrm{R} 1$ & $400-592$ & $10 / 78$ & 29 & $7 \mathrm{~S} / 7 \mathrm{E}-03 \mathrm{~A} 1$ & $250-452$ & $09 / 78$ & -90 \\
\hline $5 \mathrm{~S} / 8 \mathrm{E}-31 \mathrm{~J} 1$ & $240-302$ & $10 / 78$ & -63 & $7 \mathrm{~S} / 8 \mathrm{E}-02 \mathrm{~B} 1$ & $520-575$ & $10 / 78$ & -155 \\
\hline $5 S / 8 E-34 G 1$ & -- & $10 / 78$ & -100 & $7 \mathrm{~S} / 8 \mathrm{E}-(03 \mathrm{~A} 1$ & $400-500$ & $10 / 78$ & -149 \\
\hline 6S/6E-01G1 & $205-296$ & $10 / 78$ & -38 & 7S/8E-07R1 & -. & $10 / 78$ & -129 \\
\hline 6S/6E-01Q1 & -- & $10 / 78$ & -28 & $7 \mathrm{~S} / 8 \mathrm{E}-(09 \mathrm{M} 1$ & 54()$-60()$ & $10 / 78$ & -124 \\
\hline $6 \mathrm{~S} / 6 \mathrm{E}-12 \mathrm{G} 1$ & -- & $10 / 78$ & -38 & $7 \mathrm{~S} / 8 \mathrm{E}-17 \mathrm{~A} 1$ & $8(0)-1,100$ & $10 / 78$ & -126 \\
\hline $6 \mathrm{~S} / 6 \mathrm{E}-17 \mathrm{~K} 1$ & $480-580$ & $09 / 78$ & 794 & $7 \mathrm{~S} / 8 \mathrm{E}-17 \mathrm{~F} 1$ & $265-32.5$ & $10 / 78$ & -122 \\
\hline 6S/7E-(01H1 & $525-595$ & $10 / 78$ & -70 & $7 \mathrm{~S} / 8 \mathrm{E}-17 \mathrm{G} 1$ & $40(0-750)$ & $10 / 78$ & -122 \\
\hline $6 \mathrm{~S} / 7 \mathrm{E}-01 \mathrm{P} 1$ & -- & $10 / 78$ & -56 & $7 \mathrm{~S} / 8 \mathrm{E}-18 \mathrm{C} / \mathrm{I}$ & -- & $10 / 78$ & -120 \\
\hline 6S/7E-02G1 & $160-363$ & $10 / 78$ & -33 & $7 \mathrm{~S} / 8 \mathrm{E}-20 \mathrm{~B} 1$ & $210-501$ & $10 / 78$ & -1.31 \\
\hline 6S/7E-07B1 & $200-480$ & $10 / 78$ & -128 & $7 \mathrm{~S} / 8 \mathrm{E}-22 \mathrm{~K} 1$ & $446-775$ & $10 / 78$ & -1.36 \\
\hline 6S/7E-09L2 & $225-300$ & $10 / 78$ & -23 & $7 \mathrm{~S} / 8 \mathrm{E}-23 \mathrm{Q} 1$ & $316-416$ & $10 / 78$ & -165 \\
\hline 6S/7E-10G1 & $100-360$ & $10 / 78$ & -27 & $7 \mathrm{~S} / 8 \mathrm{E}-23 \mathrm{Q} 2$ & $365-425$ & $10 / 78$ & -165 \\
\hline $6 \mathrm{~S} / 7 \mathrm{E}-12 \mathrm{E} 1$ & $120-600$ & $10 / 78$ & -54 & $7 \mathrm{~S} / 8 \mathrm{E}-28 \mathrm{G} 1$ & $195-295$ & $09 / 78$ & -130 \\
\hline $6 \mathrm{~S} / 7 \mathrm{E}-13 \mathrm{M} 2$ & $146-386$ & $10 / 78$ & -65 & $7 \mathrm{~S} / 8 \mathrm{E}-29 \mathrm{G} 1$ & -- & $09 / 78$ & -129 \\
\hline $6 \mathrm{~S} / 7 \mathrm{E}-13 \mathrm{M} 4$ & $480-600$ & $10 / 78$ & -75 & $7 \mathrm{~S} / 8 \mathrm{E}-33 \mathrm{~B} 1$ & $343-522$ & $09 / 78$ & -1.39 \\
\hline $6 \mathrm{~S} / 7 \mathrm{E}-17 \mathrm{R} 1$ & -. & $10 / 78$ & -57 & $7 \mathrm{~S} / 8 \mathrm{E}-33 \mathrm{E} 1$ & $318-702$ & $(09 / 78$ & -117 \\
\hline $6 \mathrm{~S} / 7 \mathrm{E}-22 \mathrm{~B} 1$ & $1,088-1,365$ & $10 / 78$ & -57 & 7S/8E-34G1 & -- & $09 / 78$ & -1.34 \\
\hline $6 \mathrm{~S} / 7 \mathrm{E}-23 \mathrm{D} 3$ & $380-600$ & $10 / 78$ & -75 & $7 \mathrm{~S} / 8 \mathrm{E}-34 \mathrm{~K} 1$ & $300-895$ & $09 / 78$ & -141 \\
\hline $6 \mathrm{~S} / 7 \mathrm{E}-23 \mathrm{~F} 1$ & $312-375$ & $10 / 78$ & -76 & $7 \mathrm{~S} / 8 \mathrm{E}-35 \mathrm{~K} 1$ & -- & $(0) / 78$ & -140 \\
\hline $6 \mathrm{~S} / 8 \mathrm{E}-02 \mathrm{D} 1$ & $292-760$ & $10 / 78$ & -95 & $7 \mathrm{~S} / 9 \mathrm{E}-(03 \mathrm{D} 1$ & $320-600$ & $10 / 78$ & -175 \\
\hline $6 \mathrm{~S} / 8 \mathrm{E}-(02 \mathrm{~F} 1$ & $540-1,015$ & $10 / 78$ & -100 & $7 \mathrm{~S} / 9 \mathrm{E}-() 4 \mathrm{C} \cdot 1$ & $300-600$ & $10 / 78$ & -169 \\
\hline $6 \mathrm{~S} / 8 \mathrm{E}-03 \mathrm{C} 1$ & $508-1,140$ & $10 / 78$ & -84 & $7 \mathrm{~S} / 9 \mathrm{E}-(0.5 \mathrm{M} 1$ & $460-940$ & $10 / 78$ & -196 \\
\hline
\end{tabular}


Appendix 3: Ground-water levels, autumn 1978--Continued

\begin{tabular}{|cccc}
\hline $\begin{array}{c}\text { Well } \\
\text { number }\end{array}$ & $\begin{array}{c}\text { Perforated } \\
\text { interval } \\
\text { (depth, in feet } \\
\text { below land surface) }\end{array}$ & Date & $\begin{array}{c}\text { Altitude } \\
\text { of water } \\
\text { level }\end{array}$ \\
\hline 7S/9E-07H2 & $451-566$ & $10 / 78$ & -183 \\
7S/9E-08P1 & $430-590$ & $10 / 78$ & -192 \\
7S/9E-13N1 & $90-306$ & $10 / 78$ & -144 \\
7S/9E-16M2 & $530-590$ & $10 / 78$ & -199 \\
7S/9E-17K1 & $420-570$ & $10 / 78$ & -190 \\
& $590-620$ & & \\
7S/9E-22G2 & $830-900$ & $10 / 78$ & -201 \\
7S/9E-23N1 & $560-620$ & $10 / 78$ & -207 \\
7S/9E-26G2 & $336-560$ & $10 / 78$ & -188 \\
7S/10E-27A1 & -- & $10 / 78$ & -18 \\
8S/8E-03B1 & $485-680$ & $09 / 78$ & -142 \\
8S/8E-03L1 & $402-618$ & $09 / 78$ & -139 \\
8S/8E-11A4 & $300-380$ & $09 / 78$ & -153 \\
8S/8E-11H1 & $560-876$ & $09 / 78$ & -156 \\
8S/8E-24A1 & $800-900$ & $09 / 78$ & -162 \\
8S/8E-24A2 & -- & $09 / 78$ & -162 \\
8S/8E-24J1 & $216-312$ & $09 / 78$ & -169 \\
8S/8E-24L1 & -- & $09 / 78$ & -164 \\
8S/9E-30A1 & $315-595$ & $09 / 78$ & -168 \\
8S/9E-31Q1 & $230-350$ & $10 / 78$ & -195 \\
8S/9E-31R1 & $182-278$ & $10 / 78$ & -186 \\
8S/9E-31R2 & $180-348$ & $10 / 78$ & -181 \\
8S/9E-33N1 & -- & $09 / 78$ & -172 \\
\hline & & & \\
\hline
\end{tabular}


Appendix 4: Ground-water levels, autumn 1986

[Altitude of water level in feet above or below (-) sea level]

\begin{tabular}{cccccc}
\hline $\begin{array}{c}\text { Well } \\
\text { number }\end{array}$ & $\begin{array}{c}\text { Perforated } \\
\text { interval } \\
\text { (depth, in feet } \\
\text { below land surface) }\end{array}$ & Date & $\begin{array}{c}\text { Altitude } \\
\text { of water } \\
\text { level }\end{array}$ & $\begin{array}{c}\text { Well } \\
\text { number }\end{array}$ & $\begin{array}{c}\text { Perforated } \\
\text { interval } \\
\text { (depth, in feet } \\
\text { below land surface) }\end{array}$
\end{tabular} \begin{tabular}{c}
$\begin{array}{c}\text { Altitude } \\
\text { of water } \\
\text { level }\end{array}$ \\
\hline
\end{tabular}

\begin{tabular}{|c|c|c|c|c|c|c|c|}
\hline $2 S / 5 E-32 E 6$ & $110-180$ & $10 / 86$ & 1,010 & 4S/5E-25D1 & $540-850$ & $11 / 86$ & 102 \\
\hline $2 \mathrm{~S} / 5 \mathrm{E}-33 \mathrm{E} 5$ & $261-281$ & $10 / 86$ & 1,110 & $4 \mathrm{~S} / 5 \mathrm{E}-25 \mathrm{P} 1$ & $500-740$ & $11 / 86$ & 84 \\
\hline 3S/3E-10P1 & $476-776$ & $10 / 86$ & 922 & & $790-840$ & & \\
\hline 3S/4E-20D1 & $560-580$ & $11 / 86$ & 753 & 4S/5E-26A1 & $1,050-1,400$ & $11 / 86$ & 95 \\
\hline 3S/4E-20F1 & $600-640$ & $11 / 86$ & 758 & 4S/5E-26B1 & $1,050-1,360$ & $11 / 86$ & 107 \\
\hline $3 S / 4 E-20 F 2$ & $850-890$ & $11 / 86$ & 739 & $4 \mathrm{~S} / 5 \mathrm{E}-26 \mathrm{C} 1$ & $430-500$ & $11 / 86$ & 106 \\
\hline $3 S / 4 E-20 F 3$ & $1,100-1,140$ & $11 / 86$ & 728 & & $660-880$ & & \\
\hline 3S/4E-20J1 & $550-590$ & $11 / 86$ & 782 & 4S/5E-26D1 & $769-1,385$ & $11 / 86$ & 111 \\
\hline $3 S / 4 E-20 J 2$ & $690-730$ & $11 / 86$ & 736 & $4 \mathrm{~S} / 5 \mathrm{E}-26 \mathrm{H} 1$ & $816-1,360$ & $11 / 86$ & 101 \\
\hline $3 S / 4 E-20 J 3$ & $850-890$ & $11 / 86$ & 721 & 4S/5E-26K1 & $400-820$ & $11 / 86$ & 105 \\
\hline 3S/4E-29F1 & $550-570$ & $11 / 86$ & 748 & 4S/5E-27E1 & $344-392$ & $11 / 86$ & 129 \\
\hline 3S/4E-29R1 & $431-551$ & $11 / 86$ & 614 & 4S/5E-27E2 & $456-835$ & $11 / 86$ & 132 \\
\hline 3S/4E-32B2 & $475-611$ & $10 / 86$ & 605 & 4S/5E-28F1 & $500-925$ & $11 / 86$ & 145 \\
\hline 3S/4E-35R1 & $600-900$ & $11 / 86$ & 267 & 4S/5E-28F2 & $500-925$ & $11 / 86$ & 135 \\
\hline 3S/4E-35R2 & $600-1,000$ & $10 / 86$ & 285 & 4S/5E-29F1 & $100-407$ & $11 / 86$ & 167 \\
\hline 3S/5E-10L2 & $190-260$ & $10 / 86$ & 752 & 4S/5E-29K1 & -- & $11 / 86$ & 155 \\
\hline 3S/5E-10R1 & $177-207$ & $10 / 86$ & 894 & 4S/5E-30C1 & $300-440$ & $11 / 86$ & 175 \\
\hline 3S/5E-11Q1 & $170-236$ & $12 / 86$ & 872 & 4S/5E-34C1 & $240-500$ & $11 / 86$ & 119 \\
\hline 3S/5E-12P1 & $304-379$ & $10 / 86$ & 860 & 4S/5E-34J1 & $240-500$ & $11 / 86$ & 106 \\
\hline $3 S / 6 E-21 F 2$ & $342-430$ & $10 / 86$ & 755 & 4S/5E-35E1 & -- & $11 / 86$ & 95 \\
\hline 3S/6E-36P1 & -- & $10 / 86$ & 690 & 4S/5E-36D1 & $470-492$ & $11 / 86$ & 88 \\
\hline 4S/4E-01B3 & $400-700$ & $10 / 86$ & 260 & & $658-678$ & & \\
\hline 4S/4E-02B1 & $570-1,003$ & $10 / 86$ & 278 & 4S/5E-36L1 & $408-840$ & $11 / 86$ & 96 \\
\hline 4S/4E-13H1 & $400-500$ & $10 / 86$ & 206 & 4S/5E-36L2 & $252-650$ & $11 / 86$ & 70 \\
\hline \multirow[t]{2}{*}{ 4S/4E-13P1 } & $345-365$ & $10 / 86$ & 204 & 4S/6E-25C2 & $540-700$ & $10 / 86$ & 23 \\
\hline & $98-408$ & & & 4S/6E-25C3 & $540-700$ & $10 / 86$ & 16 \\
\hline \multirow[t]{2}{*}{ 4S/4E-15J1 } & $164-344$ & $10 / 86$ & 217 & 4S/6E-35P1 & $500-600$ & $10 / 86$ & 13 \\
\hline & $415-435$ & & & 4S/7E-29E1 & $330-700$ & $12 / 86$ & -5 \\
\hline 4S/5E-03P1 & $288-600$ & $10 / 86$ & 176 & 4S/7E-29M1 & $570-680$ & $12 / 86$ & -15 \\
\hline 4S/5E-04F1 & $276-576$ & $10 / 86$ & 196 & 4S/7E-29N2 & $118-263$ & $10 / 86$ & 2 \\
\hline \multirow[t]{3}{*}{ 4S/5E-04N1 } & $520-690$ & $09 / 86$ & 188 & 4S/7E-30E3 & $180-360$ & $10 / 86$ & 30 \\
\hline & $780-810$ & & & 4S/7E-30M1 & -- & $10 / 86$ & 14 \\
\hline & $860-890$ & & & 4S/7E-30M2 & -- & $10 / 86$ & 11 \\
\hline 4S/5E-08A1 & $368-1,100$ & $11 / 86$ & 182 & 4S/7E-30M6 & -- & $10 / 86$ & 13 \\
\hline 4S/5E-09F1 & $227-616$ & $10 / 86$ & 175 & 4S/7E-31Q3 & $236-356$ & $10 / 86$ & -2 \\
\hline 4S/5E-09F3 & $410-670$ & $10 / 86$ & 177 & & $590-710$ & & \\
\hline 4S/5E-11E1 & $172-607$ & $10 / 86$ & 151 & $4 \mathrm{~S} / 7 \mathrm{E}-32 \mathrm{G} 2$ & $280-320$ & $10 / 86$ & -5 \\
\hline \multirow[t]{2}{*}{ 4S/5E-14N1 } & $330-640$ & $10 / 86$ & 129 & & $380-400$ & & \\
\hline & $715-780$ & & & 4S/7E-32N2 & $195-462$ & $10 / 86$ & -7 \\
\hline 4S/5E-15R1 & $243-507$ & $10 / 86$ & 130 & 4S/7E-33N1 & $100-412$ & $10 / 86$ & -2 \\
\hline 4S/5E-15R2 & $400-560$ & $10 / 86$ & 139 & 4S/7E-33Q1 & $240-460$ & $10 / 86$ & -14 \\
\hline \multirow[t]{2}{*}{$4 \mathrm{~S} / 5 \mathrm{E}-21 \mathrm{~J} 2$} & $440-470$ & $11 / 86$ & 145 & 5S/4E-02G1 & $300-500$ & $10 / 86$ & 316 \\
\hline & $680-1,000$ & & & 5S/5E-01C1 & $216-350$ & $11 / 86$ & 74 \\
\hline 4S/5E-22A1 & -- & $10 / 86$ & 135 & $5 \mathrm{~S} / 5 \mathrm{E}-01 \mathrm{D} 3$ & -- & $11 / 86$ & 82 \\
\hline 4S/5E-22B1 & -- & $10 / 86$ & 137 & 5S/5E-01L5 & $500-740$ & $11 / 86$ & 69 \\
\hline
\end{tabular}


Appendix 4: Ground-water levels, autumn 1986--Continued

\begin{tabular}{|c|c|c|c|c|c|c|c|}
\hline $\begin{array}{c}\text { Well } \\
\text { number }\end{array}$ & $\begin{array}{c}\text { Perforated } \\
\text { interval } \\
\text { (depth, in feet } \\
\text { below land surface) }\end{array}$ & Date & $\begin{array}{l}\text { Altitude } \\
\text { of water } \\
\text { level }\end{array}$ & $\begin{array}{c}\text { Well } \\
\text { number }\end{array}$ & $\begin{array}{c}\text { Perforated } \\
\text { interval } \\
\text { (depth, in feet } \\
\text { below land surface) }\end{array}$ & Date & $\begin{array}{c}\text { Altitude } \\
\text { of water } \\
\text { level }\end{array}$ \\
\hline 5S/5E-01M4 & $150-502$ & $11 / 86$ & 72 & $5 S / 6 \mathrm{E}-07 \mathrm{C} 2$ & $320-500$ & $12 / 86$ & 54 \\
\hline $5 \mathrm{~S} / 5 \mathrm{E}-01 \mathrm{~N} 1$ & -- & $11 / 86$ & 72 & 5S/6E-07J1 & $460-560$ & $11 / 86$ & 47 \\
\hline $5 \mathrm{~S} / 5 \mathrm{E}-01 \mathrm{P} 1$ & $108-192$ & $11 / 86$ & 67 & 5S/6E-07M1 & -- & $12 / 86$ & 56 \\
\hline 5S/5E-01Q2 & $400-835$ & $11 / 86$ & 64 & 5S/6E-07N1 & $144-200$ & $10 / 86$ & 52 \\
\hline 5S/5E-01Q3 & $400-820$ & $11 / 86$ & 60 & $5 \mathrm{~S} / 6 \mathrm{E}-07 \mathrm{Q} 2$ & $300-500$ & $10 / 86$ & 49 \\
\hline $5 \mathrm{~S} / 5 \mathrm{E}-02 \mathrm{~A} 1$ & -- & $11 / 86$ & 82 & 5S/6E-07Q3 & $380-500$ & $10 / 86$ & 46 \\
\hline $5 S / 5 E-02 B 1$ & $450-600$ & $11 / 86$ & 81 & 5S/6E-07R1 & $364-604$ & $10 / 86$ & 51 \\
\hline $5 S / 5 E-02 F 2$ & $250-495$ & $11 / 86$ & 73 & $5 \mathrm{~S} / 6 \mathrm{E}-08 \mathrm{M} 2$ & $280-520$ & $11 / 86$ & 54 \\
\hline \multirow[t]{2}{*}{$5 S / 5 E-02 L 1$} & $130-140$ & $11 / 86$ & 83 & 5S/6E-08M3 & $650-920$ & $11 / 86$ & 41 \\
\hline & $200-220$ & & & $5 S / 6 E-08 N 2$ & $450-780$ & $12 / 86$ & 52 \\
\hline 5S/5E-11A1 & -- & $11 / 86$ & 64 & $5 \mathrm{~S} / 6 \mathrm{E}-08 \mathrm{~N} 3$ & $390-690$ & $11 / 86$ & 44 \\
\hline $5 \mathrm{~S} / 5 \mathrm{E}-11 \mathrm{~A} 3$ & $250-440$ & $11 / 86$ & 69 & 5S/6E-08P1 & -- & $12 / 86$ & 49 \\
\hline $5 \mathrm{~S} / 5 \mathrm{E}-12 \mathrm{~B} 3$ & $410-800$ & $11 / 86$ & 56 & $5 \mathrm{~S} / 6 \mathrm{E}-08 \mathrm{Q} 1$ & -- & $12 / 86$ & 43 \\
\hline $5 S / 5 E-12 C 1$ & $163-503$ & $11 / 86$ & 74 & 5S/6E-09B1 & -- & $12 / 86$ & 63 \\
\hline 5S/5E-12D1 & $125-360$ & $11 / 86$ & 65 & $5 \mathrm{~S} / 6 \mathrm{E}-09 \mathrm{Q} 1$ & $740-1,010$ & $12 / 86$ & 31 \\
\hline 5S/5E-12D2 & $278-498$ & $11 / 86$ & 58 & $5 \mathrm{~S} / 6 \mathrm{E}-10 \mathrm{D} 1$ & $530-1,030$ & $12 / 86$ & 92 \\
\hline $5 \mathrm{~S} / 5 \mathrm{E}-12 \mathrm{H} 2$ & $432-590$ & $11 / 86$ & 52 & $5 \mathrm{~S} / 6 \mathrm{E}-10 \mathrm{D} 2$ & -- & $12 / 86$ & 52 \\
\hline $5 \mathrm{~S} / 5 \mathrm{E}-12 \mathrm{~J} 1$ & $400-880$ & $11 / 86$ & 50 & $5 \mathrm{~S} / 6 \mathrm{E}-10 \mathrm{E} 1$ & $480-670$ & $12 / 86$ & 53 \\
\hline $5 S / 5 E-12 L 2$ & $208-461$ & $11 / 86$ & 68 & , & $820-925$ & & \\
\hline $5 S / 5 E-12 Q 1$ & -- & $11 / 86$ & 54 & 5S/6E-12G1 & $192-272$ & $10 / 86$ & 0 \\
\hline $5 S / 6 E-01 C 1$ & $126-1,250$ & $10 / 86$ & 14 & & $472-604$ & & \\
\hline \multirow[t]{6}{*}{ 5S/6E-01D2 } & $410-440$ & $10 / 86$ & 11 & 5S/6E-12M1 & $140-650$ & $10 / 86$ & 15 \\
\hline & $510-626$ & & & $5 \mathrm{~S} / 6 \mathrm{E}-12 \mathrm{M} 2$ & $200-500$ & $10 / 86$ & -4 \\
\hline & $678-716$ & & & $5 \mathrm{~S} / 6 \mathrm{E}-12 \mathrm{~N} 1$ & $470-946$ & $10 / 86$ & -7 \\
\hline & $774-801$ & & & $5 S / 6 E-12 Q 1$ & -- & $10 / 86$ & 12 \\
\hline & $840-902$ & & & $5 \mathrm{~S} / 6 \mathrm{E}-12 \mathrm{Q} 2$ & -- & $10 / 86$ & 10 \\
\hline & $920-980$ & & & 5S/6E-13B1 & -- & $10 / 86$ & -11 \\
\hline 5S/6E-01J1 & $500-730$ & $10 / 86$ & -8 & 5S/6E-13D1 & $518-566$ & $10 / 86$ & -10 \\
\hline 5S/6E-01J2 & $320-620$ & $10 / 86$ & -2 & & $614-878$ & & \\
\hline $5 S / 6 E-01 K 2$ & $481-601$ & $10 / 86$ & 1 & 5S/6E-13M1 & $339-369$ & $10 / 86$ & -7 \\
\hline $5 \mathrm{~S} / 6 \mathrm{E}-02 \mathrm{~A} 2$ & $450-650$ & $12 / 86$ & 8 & & $387-400$ & & \\
\hline \multirow[t]{4}{*}{$5 \mathrm{~S} / 6 \mathrm{E}-02 \mathrm{G} 1$} & $470-600$ & $12 / 86$ & -1 & & $517-626$ & & \\
\hline & $670-690$ & & & & $740-900$ & & \\
\hline & $710-770$ & & & $5 \mathrm{~S} / 6 \mathrm{E}-14 \mathrm{G} 1$ & $520-600$ & $10 / 86$ & -2 \\
\hline & $890-1,000$ & & & & $630-670$ & & \\
\hline 5S/6E-03P1 & $570-970$ & $12 / 86$ & 30 & $5 S / 6 E-14 G 2$ & -- & $10 / 86$ & -1 \\
\hline $5 \mathrm{~S} / 6 \mathrm{E}-05 \mathrm{~A} 1$ & $520-1,220$ & $12 / 86$ & 54 & $5 S / 6 E-14 Q 1$ & $440-600$ & $10 / 86$ & 0 \\
\hline 5S/6E-05B1 & $850-1,045$ & $12 / 86$ & 55 & & $706-810$ & & \\
\hline 5S/6E-05K1 & $1,036-1,300$ & $12 / 86$ & 62 & $5 S / 6 E-16 A 1$ & $360-560$ & $12 / 86$ & 23 \\
\hline 5S/6E-05M1 & $540-1,040$ & $12 / 86$ & 55 & $5 \mathrm{~S} / 6 \mathrm{E}-16 \mathrm{~A} 2$ & $445-530$ & $12 / 86$ & 33 \\
\hline \multirow[t]{3}{*}{ 5S/6E-05Q1 } & $525-730$ & $11 / 86$ & 47 & & $730-965$ & & \\
\hline & $800-890$ & & & $5 \mathrm{~S} / 6 \mathrm{E}-16 \mathrm{H} 1$ & $336-552$ & $12 / 86$ & 29 \\
\hline & $1,020-1,135$ & & & $5 \mathrm{~S} / 6 \mathrm{E}-16 \mathrm{~K} 1$ & $302-366$ & $12 / 86$ & 25 \\
\hline 5S/6E-06B3 & $455-890$ & $12 / 86$ & 71 & $5 \mathrm{~S} / 6 \mathrm{E}-16 \mathrm{M} 1$ & $350-500$ & $12 / 86$ & 29 \\
\hline $5 S / 6 \mathrm{E}-06 \mathrm{C} 1$ & -- & $11 / 86$ & 74 & $5 S / 6 E-16 N 2$ & $564-904$ & $12 / 86$ & 23 \\
\hline $5 S / 6 E-06 C 2$ & -- & $11 / 86$ & 77 & $5 S / 6 E-16 Q 1$ & $156-300$ & $12 / 86$ & 22 \\
\hline 5S/6E-06Q1 & $668-1,202$ & $10 / 86$ & 51 & $5 S / 6 E-16 Q 2$ & $242-354$ & $12 / 86$ & 15 \\
\hline 5S/6E-07B1 & -- & $10 / 86$ & 50 & $5 \mathrm{~S} / 6 \mathrm{E}-17 \mathrm{~F} 1$ & $290-590$ & $12 / 86$ & 42 \\
\hline
\end{tabular}


Appendix 4: Ground-water levels, autumn 1986--Continued

\begin{tabular}{|c|c|c|c|c|c|c|c|}
\hline $\begin{array}{c}\text { Well } \\
\text { number }\end{array}$ & $\begin{array}{c}\text { Perforated } \\
\text { interval } \\
\text { (depth, in feet } \\
\text { below land surface) }\end{array}$ & Date & $\begin{array}{c}\text { Altitude } \\
\text { of water } \\
\text { level }\end{array}$ & $\begin{array}{c}\text { Well } \\
\text { number }\end{array}$ & $\begin{array}{c}\text { Perforated } \\
\text { interval } \\
\text { (depth, in feet } \\
\text { below land surface) }\end{array}$ & Date & $\begin{array}{c}\text { Altitude } \\
\text { of water } \\
\text { level }\end{array}$ \\
\hline $5 S / 6 E-17 G 2$ & $550-890$ & $12 / 86$ & 32 & $5 S / 6 E-24 D 1$ & -- & $12 / 86$ & -14 \\
\hline $5 S / 6 \mathrm{E}-17 \mathrm{M} 2$ & $376-700$ & $12 / 86$ & 35 & $5 S / 6 E-24 F 1$ & $440-690$ & $12 / 86$ & 2 \\
\hline $5 S / 6 E-17 P 2$ & $420-700$ & $12 / 86$ & 36 & $5 \mathrm{~S} / 6 \mathrm{E}-24 \mathrm{G} 1$ & $480-680$ & $12 / 86$ & -13 \\
\hline $5 S / 6 E-18 C 2$ & $300-650$ & $12 / 86$ & 51 & & $810-930$ & & \\
\hline $5 \mathrm{~S} / 6 \mathrm{E}-18 \mathrm{C} 3$ & -- & $12 / 86$ & 37 & $5 \mathrm{~S} / 6 \mathrm{E}-24 \mathrm{M} 1$ & $396-564$ & $12 / 86$ & -10 \\
\hline $5 \mathrm{~S} / 6 \mathrm{E}-18 \mathrm{G} 1$ & $377-497$ & $12 / 86$ & 32 & & $604-628$ & & \\
\hline $5 \mathrm{~S} / 6 \mathrm{E}-18 \mathrm{~L} 2$ & $317-509$ & $12 / 86$ & 34 & & $668-778$ & & \\
\hline 5S/6E-18R1 & $296-450$ & $12 / 86$ & 30 & 5S/6E-24R1 & -- & $12 / 86$ & -14 \\
\hline 5S/6E-18R2 & $425-800$ & $12 / 86$ & 29 & $5 \mathrm{~S} / 6 \mathrm{E}-25 \mathrm{~A} 1$ & $400-656$ & $12 / 86$ & -8 \\
\hline $5 S / 6 E-20 A 1$ & $435-522$ & $12 / 86$ & 28 & $5 \mathrm{~S} / 6 \mathrm{E}-25 \mathrm{~A} 2$ & $430-650$ & $12 / 86$ & -28 \\
\hline $5 S / 6 \mathrm{E}-20 \mathrm{~F} 3$ & $628-925$ & $12 / 86$ & 45 & $5 S / 6 E-27 C 1$ & $138-292$ & $12 / 86$ & 42 \\
\hline 5S/6E-20P1 & $300-588$ & $12 / 86$ & 31 & $5 S / 6 E-27 C 2$ & -- & $12 / 86$ & 32 \\
\hline $5 \mathrm{~S} / 6 \mathrm{E}-21 \mathrm{G} 1$ & $167-237$ & $12 / 86$ & 30 & 5S/6E-27D1 & $266-392$ & $12 / 86$ & 32 \\
\hline $5 S / 6 E-21 G 3$ & $195-500$ & $12 / 86$ & 17 & $5 S / 6 E-27 D 2$ & $170-370$ & $12 / 86$ & 23 \\
\hline $5 \mathrm{~S} / 6 \mathrm{E}-21 \mathrm{H} 3$ & $210-550$ & $12 / 86$ & 23 & $5 S / 6 E-28 C 1$ & $204-252$ & $12 / 86$ & 29 \\
\hline $5 \mathrm{~S} / 6 \mathrm{E}-21 \mathrm{H} 5$ & $400-600$ & $12 / 86$ & 29 & $5 S / 6 E-28 C 2$ & $410-680$ & $12 / 86$ & 28 \\
\hline 5S/6E-21J1 & $172-278$ & $12 / 86$ & 19 & $5 \mathrm{~S} / 6 \mathrm{E}-28 \mathrm{E} 2$ & $320-470$ & $12 / 86$ & 38 \\
\hline $5 \mathrm{~S} / 6 \mathrm{E}-21 \mathrm{~N} 2$ & $400-820$ & $12 / 86$ & 36 & $5 \mathrm{~S} / 6 \mathrm{E}-28 \mathrm{H} 1$ & $194-373$ & $12 / 86$ & 47 \\
\hline $5 \mathrm{~S} / 6 \mathrm{E}-21 \mathrm{P} 1$ & $212-433$ & $12 / 86$ & 26 & $5 S / 6 E-29 B 1$ & $351-615$ & $12 / 86$ & 16 \\
\hline $5 S / 6 E-21 Q 3$ & $350-700$ & $12 / 86$ & 28 & $5 S / 6 E-29 C 1$ & $243-531$ & $12 / 86$ & 29 \\
\hline $5 S / 6 E-21 R 1$ & $250-480$ & $12 / 86$ & 28 & $5 S / 6 E-29 C 2$ & $530-670$ & $12 / 86$ & 29 \\
\hline \multirow[t]{3}{*}{$5 S / 6 E-22 B 1$} & $350-390$ & $12 / 86$ & 15 & $5 \mathrm{~S} / 6 \mathrm{E}-29 \mathrm{H} 1$ & -- & $12 / 86$ & 36 \\
\hline & $416-452$ & & & 5S/6E-29M1 & $457-723$ & $12 / 86$ & 34 \\
\hline & $468-502$ & & & $5 \mathrm{~S} / 6 \mathrm{E}-29 \mathrm{P} 1$ & $500-700$ & $12 / 86$ & 31 \\
\hline \multirow[t]{2}{*}{$5 S / 6 E-22 B 2$} & $548-704$ & $12 / 86$ & 18 & $5 \mathrm{~S} / 6 \mathrm{E}-32 \mathrm{~B} 1$ & $450-700$ & $12 / 86$ & 50 \\
\hline & $752-872$ & & & $5 S / 6 E-32 B 2$ & $460-700$ & $12 / 86$ & 43 \\
\hline $5 S / 6 E-22 G 2$ & $288-474$ & $12 / 86$ & 19 & $5 S / 6 E-32 B 3$ & $460-652$ & $12 / 86$ & 39 \\
\hline $5 \mathrm{~S} / 6 \mathrm{E}-22 \mathrm{H} 2$ & $772-855$ & $12 / 86$ & 25 & $5 S / 6 E-32 G 1$ & $459-736$ & $12 / 86$ & 81 \\
\hline \multirow[t]{4}{*}{$5 S / 6 E-22 J 1$} & $342-372$ & $12 / 86$ & 32 & $5 \mathrm{~S} / 7 \mathrm{E}-03 \mathrm{~K} 1$ & $580-740$ & $10 / 86$ & -51 \\
\hline & $388-392$ & & & $5 S / 7 E-04 A 1$ & $147-367$ & $10 / 86$ & -8 \\
\hline & $416-518$ & & & 5S/7E-04C1 & $520-800$ & $10 / 86$ & -13 \\
\hline & $524-554$ & & & 5S/7E-04D1 & $100-370$ & $10 / 86$ & -8 \\
\hline \multirow[t]{2}{*}{ 5S/6E-22M1 } & $222-260$ & $12 / 86$ & 26 & $5 \mathrm{~S} / 7 \mathrm{E}-04 \mathrm{H} 1$ & $570-840$ & $10 / 86$ & -41 \\
\hline & $287-340$ & & & 5S/7E-04M1 & $192-678$ & $10 / 86$ & -10 \\
\hline \multirow[t]{3}{*}{ 5S/6E-23E2 } & $374-383$ & $12 / 86$ & -1 & 5S/7E-04N1 & $147-411$ & $10 / 86$ & -16 \\
\hline & $400-413$ & & & 5S/7E-04Q1 & $123-363$ & $10 / 86$ & -16 \\
\hline & $482-509$ & & & 5S/7E-04Q3 & $240-320$ & $10 / 86$ & -24 \\
\hline 5S/6E-23E3 & $275-420$ & $12 / 86$ & 22 & $5 S / 7 E-05 C 1$ & $260-400$ & $10 / 86$ & -6 \\
\hline 5S/6E-23F1 & $778-794$ & $12 / 86$ & 19 & 5S/7E-05K1 & -- & $10 / 86$ & -18 \\
\hline $5 \mathrm{~S} / 6 \mathrm{E}-23 \mathrm{~K} 2$ & $476-492$ & $12 / 86$ & 7 & 5S/7E-05R1 & $230-350$ & $10 / 86$ & -26 \\
\hline \multirow[t]{3}{*}{ 5S/6E-23L1 } & $110-168$ & $12 / 86$ & 7 & 5S/7E-06B1 & $168-180$ & $10 / 86$ & 2 \\
\hline & $280-352$ & & & & $280-303$ & & \\
\hline & $355-426$ & & & 5S/7E-06B3 & $300-660$ & $10 / 86$ & -8 \\
\hline $5 \mathrm{~S} / 6 \mathrm{E}-23 \mathrm{~L} 3$ & $255-312$ & $12 / 86$ & 4 & 5S/7E-06H1 & $400-480$ & $10 / 86$ & -9 \\
\hline \multirow[t]{2}{*}{ 5S/6E-23M1 } & $384-480$ & $12 / 86$ & 25 & 5S/7E-06M1 & $195-363$ & $10 / 86$ & -3 \\
\hline & $576-776$ & & & & $555-651$ & & \\
\hline 5S/6E-23N1 & $504-577$ & $12 / 86$ & 31 & 5S/7E-07F1 & $147-459$ & $12 / 86$ & -7 \\
\hline
\end{tabular}


Appendix 4: Ground-water levels, autumn 1986--Continued

\begin{tabular}{|c|c|c|c|c|c|c|c|}
\hline $\begin{array}{c}\text { Well } \\
\text { number }\end{array}$ & $\begin{array}{c}\text { Perforated } \\
\text { interval } \\
\text { (depth, in feet } \\
\text { below land surface) }\end{array}$ & Date & $\begin{array}{c}\text { Altitude } \\
\text { of water } \\
\text { level }\end{array}$ & $\begin{array}{c}\text { Well } \\
\text { number }\end{array}$ & $\begin{array}{c}\text { Perforated } \\
\text { interval } \\
\text { (depth, in feet } \\
\text { below land surface) }\end{array}$ & Date & $\begin{array}{c}\text { Altitude } \\
\text { of water } \\
\text { level }\end{array}$ \\
\hline 5S/7E-07P1 & $144-450$ & $12 / 86$ & -10 & 7S/8E-08N1 & $300-360$ & $10 / 86$ & -132 \\
\hline $5 S / 7 E-08 A 2$ & $286-650$ & $10 / 86$ & -26 & 7S/8E-09M1 & $540-600$ & $10 / 86$ & -134 \\
\hline 5S/7E-08G1 & -- & $12 / 86$ & -10 & 7S/8E-17A1 & $800-1,100$ & $10 / 86$ & -140 \\
\hline 5S/7E-08Q1 & $203-654$ & $12 / 86$ & -16 & $7 \mathrm{~S} / 8 \mathrm{E}-17 \mathrm{~F} 1$ & $265-325$ & $10 / 86$ & -138 \\
\hline $5 \mathrm{~S} / 7 \mathrm{E}-09 \mathrm{~F} 1$ & $130-310$ & $10 / 86$ & -17 & $7 \mathrm{~S} / 8 \mathrm{E}-17 \mathrm{G} 1$ & $400-750$ & $10 / 86$ & -136 \\
\hline $5 S / 7 \mathrm{E}-09 \mathrm{~K} 1$ & $147-387$ & $10 / 86$ & -22 & $7 \mathrm{~S} / 8 \mathrm{E}-18 \mathrm{C} 1$ & -- & $10 / 86$ & -132 \\
\hline 5S/7E-09L2 & $147-319$ & $10 / 86$ & -16 & 7S/8E-18Q1 & $300-500$ & $10 / 86$ & -146 \\
\hline $5 S / 7 E-10 D 2$ & $200-530$ & $10 / 86$ & -20 & 7S/8E-19G1 & -- & $10 / 86$ & -206 \\
\hline $5 S / 7 E-10 E 1$ & $70-360$ & $10 / 86$ & -22 & $7 \mathrm{~S} / 8 \mathrm{E}-20 \mathrm{~B} 1$ & $210-501$ & $10 / 86$ & -145 \\
\hline $5 S / 7 E-16 C 1$ & $147-355$ & $10 / 86$ & -21 & $7 \mathrm{~S} / 8 \mathrm{E}-20 \mathrm{H} 1$ & $260-486$ & $10 / 86$ & -134 \\
\hline $5 \mathrm{~S} / 7 \mathrm{E}-16 \mathrm{~K} 2$ & $200-415$ & $10 / 86$ & -26 & $7 \mathrm{~S} / 8 \mathrm{E}-22 \mathrm{~K} 1$ & $446-775$ & $10 / 86$ & -152 \\
\hline 5S/7E-17E1 & $459-603$ & $12 / 86$ & -23 & $7 \mathrm{~S} / 8 \mathrm{E}-23 \mathrm{Q} 2$ & $365-425$ & $10 / 86$ & -188 \\
\hline $5 S / 7 E-17 L 1$ & $212-600$ & $12 / 86$ & -29 & $7 \mathrm{~S} / 8 \mathrm{E}-28 \mathrm{G} 1$ & $195-295$ & $10 / 86$ & -149 \\
\hline 5S/7E-18D1 & $160-200$ & $12 / 86$ & -11 & 7S/8E-29D1 & $360-650$ & $10 / 86$ & -128 \\
\hline 5S/7E-18F1 & -- & $12 / 86$ & -8 & 7S/8E-29G1 & -- & $10 / 86$ & -146 \\
\hline $5 \mathrm{~S} / 7 \mathrm{E}-18 \mathrm{M} 2$ & $168-264$ & $12 / 86$ & -18 & $7 \mathrm{~S} / 8 \mathrm{E}-31 \mathrm{R} 1$ & -- & $10 / 86$ & -51 \\
\hline \multirow[t]{3}{*}{ 5S/7E-19D1 } & $440-650$ & $12 / 86$ & -18 & $7 \mathrm{~S} / 8 \mathrm{E}-33 \mathrm{~B} 1$ & $243-522$ & $10 / 86$ & -148 \\
\hline & $710-770$ & & & 7S/8E-33E1 & $318-702$ & $10 / 86$ & -142 \\
\hline & $840-920$ & & & $7 \mathrm{~S} / 8 \mathrm{E}-33 \mathrm{~N} 2$ & -- & $10 / 86$ & -157 \\
\hline $5 S / 7 E-19 H 2$ & $460-660$ & $12 / 86$ & -26 & $7 \mathrm{~S} / 8 \mathrm{E}-34 \mathrm{G} 1$ & -- & $10 / 86$ & -150 \\
\hline 5S/7E-20P2 & $150-350$ & $12 / 86$ & -14 & $7 \mathrm{~S} / 8 \mathrm{E}-34 \mathrm{~K} 1$ & $300-895$ & $10 / 86$ & -158 \\
\hline $5 S / 7 E-21 F 2$ & $82-614$ & $10 / 86$ & -36 & $7 \mathrm{~S} / 8 \mathrm{E}-35 \mathrm{~K} 1$ & -- & $10 / 86$ & -144 \\
\hline 5S/7E-21Q1 & -- & $10 / 86$ & -44 & 7S/9E-03D1 & $320-600$ & $10 / 86$ & -180 \\
\hline $5 \mathrm{~S} / 7 \mathrm{E}-22 \mathrm{H} 2$ & $506-1,100$ & $10 / 86$ & -66 & $7 \mathrm{~S} / 9 \mathrm{E}-04 \mathrm{C} 1$ & $300-600$ & $10 / 86$ & -165 \\
\hline 5S/7E-23D2 & $483-882$ & $10 / 86$ & -68 & 7S/9E-04K1 & $300-600$ & $10 / 86$ & -174 \\
\hline 5S/7E-24M4 & $250-660$ & $10 / 86$ & -64 & 7S/9E-05M1 & $460-940$ & $10 / 86$ & -181 \\
\hline 5S/7E-26E3 & $515-1,110$ & $10 / 86$ & -78 & $7 \mathrm{~S} / 9 \mathrm{E}-07 \mathrm{H} 2$ & $451-566$ & $10 / 86$ & -188 \\
\hline 5S/7E-27B1 & $236-500$ & $12 / 86$ & -33 & 7S/9E-07J1 & $410-470$ & $10 / 86$ & -189 \\
\hline 5S/7E-28E1 & $128-198$ & $12 / 86$ & -31 & & $500-570$ & & \\
\hline 5S/7E-29K1 & -- & $12 / 86$ & -39 & & $830-870$ & & \\
\hline \multirow[t]{2}{*}{$5 S / 7 E-30 C 2$} & $115-235$ & $12 / 86$ & -30 & 7S/9E-08P1 & $430-590$ & $10 / 86$ & -205 \\
\hline & $427-489$ & & & $7 \mathrm{~S} / 9 \mathrm{E}-13 \mathrm{~N} 1$ & $90-306$ & $10 / 86$ & -149 \\
\hline 5S/7E-30J1 & $500-900$ & $12 / 86$ & -38 & $7 \mathrm{~S} / 9 \mathrm{E}-16 \mathrm{M} 2$ & $530-590$ & $10 / 86$ & -213 \\
\hline 6S/6E-06M1 & $264-364$ & $12 / 86$ & 772 & 7S/9E-17K1 & $420-570$ & $10 / 86$ & -211 \\
\hline 6S/6E-17K1 & $480-580$ & $12 / 86$ & 764 & & $590-620$ & & \\
\hline 6S/8E-05R2 & $540-750$ & $10 / 86$ & -92 & & $830-900$ & & \\
\hline 6S/8E-05R3 & $520-650$ & $10 / 86$ & -99 & $7 \mathrm{~S} / 9 \mathrm{E}-22 \mathrm{G} 2$ & $560-620$ & $10 / 86$ & -203 \\
\hline $6 \mathrm{~S} / 8 \mathrm{E}-22 \mathrm{~K} 1$ & $500-1,030$ & $11 / 86$ & -123 & $7 \mathrm{~S} / 9 \mathrm{E}-23 \mathrm{~N} 1$ & $530-560$ & $10 / 86$ & -215 \\
\hline 6S/8E-25P1 & $478-658$ & $10 / 86$ & -155 & $7 \mathrm{~S} / 9 \mathrm{E}-26 \mathrm{G} 2$ & $336-432$ & $10 / 86$ & -200 \\
\hline 6S/8E-34C1 & $447-545$ & $11 / 86$ & -137 & 7S/9E-30M1 & -- & $10 / 86$ & -203 \\
\hline 6S/8E-35J1 & $514-564$ & $11 / 86$ & -147 & $7 \mathrm{~S} / 10 \mathrm{E}-27 \mathrm{~A} 1$ & -- & $10 / 86$ & -16 \\
\hline 6S/9E-33K1 & $240-402$ & $10 / 86$ & -157 & 8S/8E-03B1 & $485-680$ & $10 / 86$ & -166 \\
\hline 7S/7E-01C1 & $240-380$ & $12 / 86$ & -112 & $8 \mathrm{~S} / 8 \mathrm{E}-03 \mathrm{~L} 1$ & $402-618$ & $10 / 86$ & -163 \\
\hline 7S/7E-03A1 & $250-452$ & $10 / 86$ & -104 & $8 \mathrm{~S} / 8 \mathrm{E}-05 \mathrm{H} 1$ & $250-560$ & $10 / 86$ & -236 \\
\hline 7S/8E-02B1 & $520-575$ & $10 / 86$ & -157 & $8 \mathrm{~S} / 8 \mathrm{E}-11 \mathrm{~A} 4$ & $300-380$ & $10 / 86$ & -176 \\
\hline $7 \mathrm{~S} / 8 \mathrm{E}-03 \mathrm{~A} 1$ & $400-500$ & $10 / 86$ & -154 & $8 \mathrm{~S} / 8 \mathrm{E}-11 \mathrm{H} 1$ & $560-876$ & $10 / 86$ & -176 \\
\hline 7S/8E-07R1 & -- & $10 / 86$ & -134 & 8S/8E-15G1 & -- & $10 / 86$ & -77 \\
\hline
\end{tabular}


Appendix 5: Dissolved-solids concentration in water from selected wells

[mg/L, milligrams per liter, -, no data]

\begin{tabular}{|c|c|c|c|c|c|c|c|c|c|}
\hline $\begin{array}{c}\text { Well } \\
\text { number }\end{array}$ & $\begin{array}{c}\text { Land- } \\
\text { surface } \\
\text { altitude } \\
\text { (feet above } \\
\text { or below (-) } \\
\text { sea level) }\end{array}$ & $\begin{array}{l}\text { Perforated } \\
\text { interval } \\
\text { (depth, in } \\
\text { feet } \\
\text { below land } \\
\text { surface) }\end{array}$ & Date & $\begin{array}{l}\text { Dis- } \\
\text { solved- } \\
\text { solids } \\
\text { concen- } \\
\text { tration } \\
(\mathrm{mg} / \mathrm{L})\end{array}$ & $\begin{array}{c}\text { Well } \\
\text { number }\end{array}$ & $\begin{array}{c}\text { Land- } \\
\text { surface } \\
\text { altitude } \\
\text { (feet above } \\
\text { or below (-) } \\
\text { sea level) }\end{array}$ & $\begin{array}{l}\text { Perforated } \\
\text { interval } \\
\text { (depth, in } \\
\text { feet } \\
\text { below land } \\
\text { surface) }\end{array}$ & Date & $\begin{array}{l}\text { Dis- } \\
\text { solved- } \\
\text { solids } \\
\text { concen- } \\
\text { tration } \\
(\mathrm{mg} / \mathrm{L})\end{array}$ \\
\hline \multirow[t]{2}{*}{ 3S/3E-10P1 } & 1,170 & $476-776$ & $04 / 74$ & 265 & \multirow{2}{*}{\multicolumn{2}{|c|}{$3 \mathrm{~S} / 4 \mathrm{E}-20 \mathrm{~F} 2-$-Continued }} & & $11 / 87$ & 204 \\
\hline & & & $07 / 74$ & 296 & & & & $03 / 88$ & 218 \\
\hline \multirow[t]{22}{*}{ 3S/4E-20D1 } & 910 & $560-580$ & $06 / 77$ & 369 & & & & $11 / 88$ & 226 \\
\hline & & & $10 / 77$ & 240 & & & & $04 / 89$ & 224 \\
\hline & & & $04 / 79$ & 157 & & & & $11 / 89$ & 213 \\
\hline & & & $06 / 79$ & 119 & & & & $04 / 90$ & 233 \\
\hline & & & $09 / 79$ & 268 & $3 \mathrm{~S} / 4 \mathrm{E}-20 \mathrm{~F} 3$ & 890 & $1,100-1,140$ & $01 / 81$ & 564 \\
\hline & & & $12 / 79$ & 136 & & & & $10 / 81$ & 439 \\
\hline & & & $03 / 81$ & 307 & & & & $04 / 82$ & 662 \\
\hline & & & $10 / 81$ & 325 & & & & $10 / 82$ & 534 \\
\hline & & & $03 / 82$ & 108 & & & & $05 / 83$ & 697 \\
\hline & & & $11 / 82$ & 290 & & & & $11 / 83$ & 557 \\
\hline & & & $05 / 83$ & 196 & & & & $11 / 84$ & 464 \\
\hline & & & $11 / 83$ & 208 & & & & $04 / 85$ & 442 \\
\hline & & & $04 / 84$ & 229 & & & & $11 / 85$ & 310 \\
\hline & & & $10 / 84$ & 234 & & & & $05 / 86$ & 170 \\
\hline & & & $04 / 85$ & 291 & & & & $05 / 86$ & 169 \\
\hline & & & $11 / 85$ & 513 & & & & $12 / 86$ & 162 \\
\hline & & & $05 / 86$ & 588 & & & & $04 / 87$ & 174 \\
\hline & & & $11 / 86$ & 552 & & & & $11 / 87$ & 175 \\
\hline & & & $04 / 87$ & 552 & & & & $03 / 88$ & 161 \\
\hline & & & $11 / 87$ & 567 & & & & $11 / 88$ & 171 \\
\hline & & & $03 / 88$ & 562 & & & & $04 / 89$ & 167 \\
\hline & & & $11 / 88$ & 488 & & & & $11 / 89$ & 161 \\
\hline \multirow[t]{20}{*}{$3 S / 4 E-20 F 1$} & 890 & $600-640$ & $01 / 81$ & 409 & & & & $04 / 90$ & 147 \\
\hline & & & $10 / 81$ & 310 & $3 \mathrm{~S} / 4 \mathrm{E}-20 \mathrm{~J} 1$ & 840 & $550-590$ & $01 / 81$ & 448 \\
\hline & & & $04 / 82$ & 361 & & & & $10 / 81$ & 418 \\
\hline & & & $10 / 82$ & 444 & & & & $04 / 82$ & 388 \\
\hline & & & $05 / 83$ & 578 & & & & $10 / 82$ & 434 \\
\hline & & & $11 / 83$ & 533 & & & & $05 / 83$ & 363 \\
\hline & & & $07 / 84$ & 483 & & & & $11 / 83$ & 356 \\
\hline & & & $11 / 84$ & 656 & & & & $04 / 84$ & 360 \\
\hline & & & $04 / 85$ & 547 & & & & $12 / 84$ & 365 \\
\hline & & & $11 / 85$ & 513 & & & & $04 / 85$ & 495 \\
\hline & & & $05 / 86$ & 545 & & & & $11 / 85$ & 560 \\
\hline & & & $05 / 86$ & 544 & & & & $05 / 86$ & 567 \\
\hline & & & $12 / 86$ & 538 & & & & $05 / 86$ & 548 \\
\hline & & & $04 / 87$ & 543 & & & & $12 / 86$ & 487 \\
\hline & & & $11 / 87$ & 561 & & & & $04 / 87$ & 530 \\
\hline & & & $03 / 88$ & 556 & & & & $11 / 87$ & 548 \\
\hline & & & $11 / 88$ & 521 & & & & $03 / 88$ & 565 \\
\hline & & & $04 / 89$ & 547 & & & & $11 / 88$ & 558 \\
\hline & & & $11 / 89$ & 573 & & & & $04 / 89$ & 509 \\
\hline & & & $04 / 90$ & 577 & & & & $11 / 89$ & 559 \\
\hline \multirow[t]{13}{*}{$3 S / 4 \mathrm{E}-20 \mathrm{~F} 2$} & 890 & $850-890$ & $10 / 81$ & 249 & & & & $04 / 90$ & 555 \\
\hline & & & $04 / 82$ & 248 & $3 \mathrm{~S} / 4 \mathrm{E}-20 \mathrm{~J} 2$ & 840 & $690-730$ & $10 / 81$ & 149 \\
\hline & & & $10 / 82$ & 243 & & & & $04 / 82$ & 153 \\
\hline & & & $05 / 83$ & 233 & & & & $10 / 82$ & 148 \\
\hline & & & $11 / 83$ & 233 & & & & $05 / 83$ & 221 \\
\hline & & & $07 / 84$ & 226 & & & & $11 / 83$ & 275 \\
\hline & & & $11 / 84$ & 228 & & & & $04 / 84$ & 256 \\
\hline & & & $04 / 85$ & 233 & & & & $12 / 84$ & 222 \\
\hline & & & $11 / 85$ & 217 & & & & $04 / 85$ & 219 \\
\hline & & & $05 / 86$ & 254 & & & & $11 / 85$ & 387 \\
\hline & & & $12 / 86$ & 229 & & & & $05 / 86$ & 359 \\
\hline & & & $04 / 87$ & 192 & & & & $12 / 86$ & 426 \\
\hline & & & & & & & & $04 / 87$ & 455 \\
\hline
\end{tabular}


Appendix 5: Dissolved-solids concentration in water from selected wells--Continued

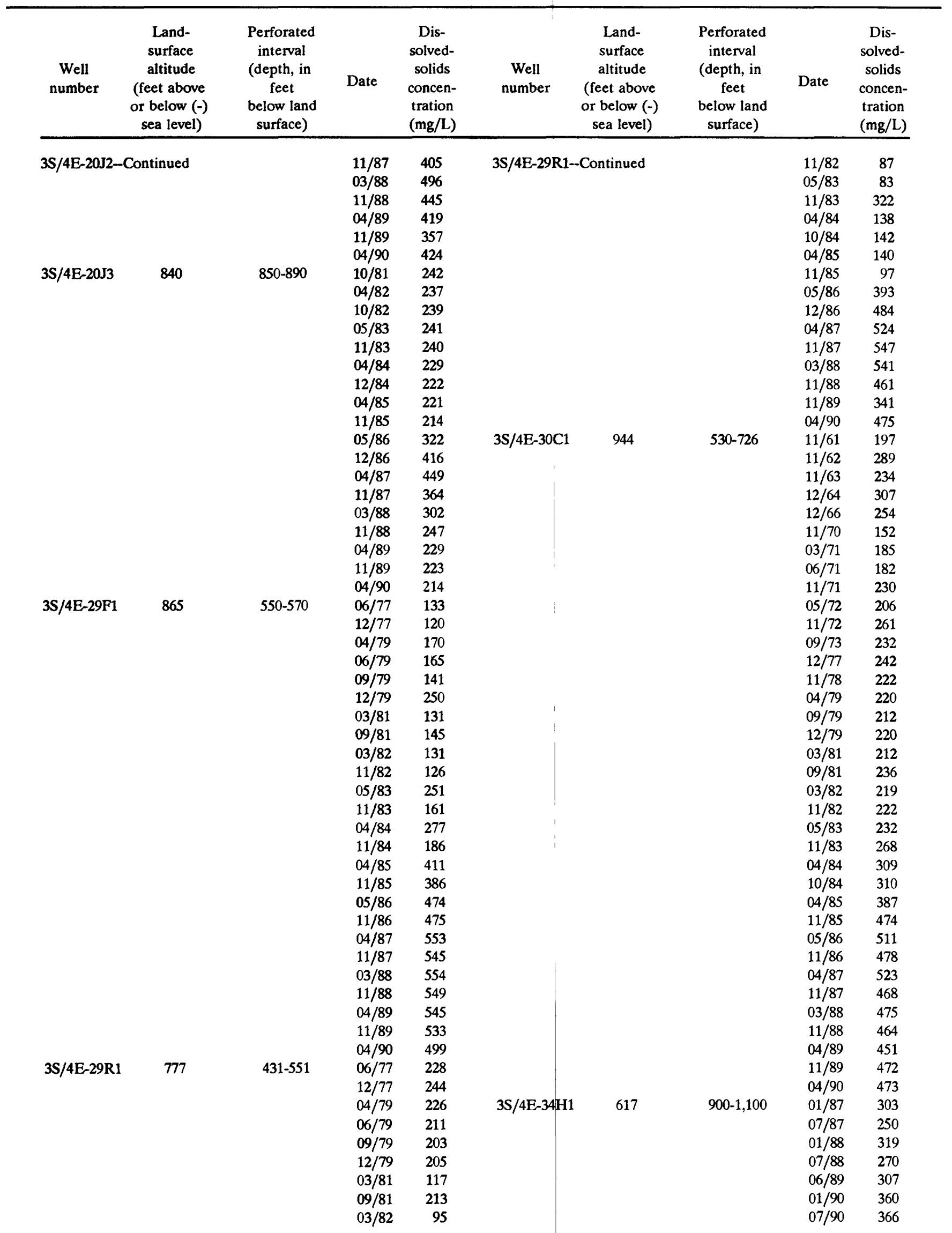


Appendix 5: Dissolved-solids concentration in water from selected wells--Continued

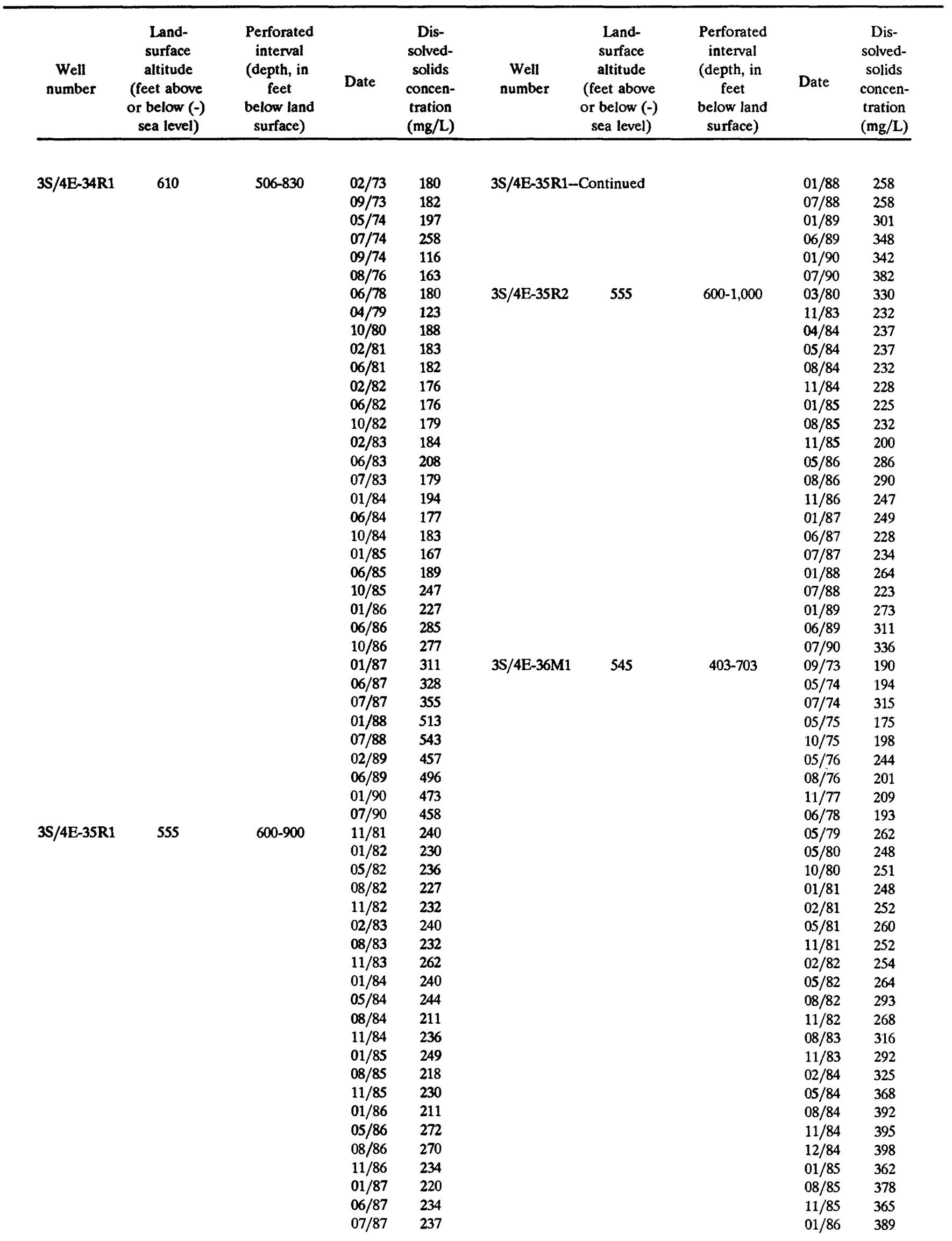


Appendix 5: Dissolved-solids concentration in water from selected wells--Continued

\begin{tabular}{|c|c|c|c|c|c|c|c|c|c|}
\hline \multirow{11}{*}{\multicolumn{2}{|c|}{ 3S/4E-36M1--Continued }} & & $08 / 86$ & 523 & \multirow{10}{*}{\multicolumn{2}{|c|}{ 4S/4E-2B1--Continued }} & & $01 / 86$ & 221 \\
\hline & & & $11 / 86$ & 369 & & & & $06 / 86$ & 274 \\
\hline & & & $01 / 87$ & 318 & & & & $10 / 86$ & 234 \\
\hline & & & $06 / 87$ & 477 & & & & $01 / 87$ & 246 \\
\hline & & & $07 / 87$ & 540 & & & & $06 / 87$ & 260 \\
\hline & & & $01 / 88$ & 437 & & & & $07 / 87$ & 287 \\
\hline & & & $07 / 88$ & 414 & & & & $07 / 88$ & 313 \\
\hline & & & $01 / 89$ & 415 & & & & $06 / 89$ & 372 \\
\hline & & & $06 / 89$ & 348 & & & & $01 / 90$ & 406 \\
\hline & & & $01 / 90$ & 450 & & & & $07 / 90$ & 402 \\
\hline & & & $07 / 90$ & 468 & 4S/4E-11K1 & 493 & - & $05 / 73$ & 314 \\
\hline \multirow[t]{16}{*}{$4 \mathrm{~S} / 4 \mathrm{E}-1 \mathrm{~N} 2$} & 500 & $525-800$ & $05 / 73$ & 213 & & & & $01 / 78$ & 267 \\
\hline & & & $09 / 73$ & 266 & & & & $01 / 81$ & 260 \\
\hline & & & $05 / 74$ & 226 & 4S/4E-11Q1 & 470 & $302-402$ & $05 / 73$ & 332 \\
\hline & & & $08 / 74$ & 271 & & & & $09 / 73$ & 276 \\
\hline & & & $09 / 74$ & 234 & & & & $09 / 74$ & 234 \\
\hline & & & $05 / 75$ & 214 & & & & $05 / 75$ & 251 \\
\hline & & & $10 / 75$ & 185 & & & & $07 / 75$ & 327 \\
\hline & & & $05 / 76$ & 196 & & & & $09 / 76$ & 289 \\
\hline & & & $09 / 76$ & 176 & & & & $05 / 77$ & 305 \\
\hline & & & $01 / 78$ & 198 & & & & $11 / 77$ & 259 \\
\hline & & & $05 / 80$ & 218 & & & & $06 / 78$ & 289 \\
\hline & & & $01 / 83$ & 214 & & & & $10 / 78$ & 295 \\
\hline & & & $06 / 83$ & 209 & & & & $05 / 79$ & 308 \\
\hline & & & $07 / 83$ & 214 & 1 & & & $05 / 80$ & 308 \\
\hline & & & $02 / 84$ & 212 & & & & $08 / 80$ & 362 \\
\hline & & & $06 / 84$ & 225 & & & & $10 / 80$ & 302 \\
\hline \multirow[t]{29}{*}{ 4S/4E-2B1 } & 565 & $570-1,003$ & $01 / 73$ & 229 & & & & $08 / 81$ & 332 \\
\hline & & & $05 / 74$ & 218 & & & & $11 / 81$ & 324 \\
\hline & & & $06 / 74$ & 253 & & & & $01 / 82$ & 318 \\
\hline & & & $09 / 74$ & 203 & & & & $05 / 82$ & 305 \\
\hline & & & $05 / 75$ & 193 & & & & $10 / 82$ & 310 \\
\hline & & & $10 / 75$ & 196 & & & & $02 / 83$ & 310 \\
\hline & & & $05 / 76$ & 202 & & & & $06 / 83$ & 262 \\
\hline & & & $08 / 76$ & 196 & & & & $07 / 83$ & 324 \\
\hline & & & $09 / 76$ & 182 & & & & $10 / 83$ & 321 \\
\hline & & & $05 / 77$ & 189 & & & & $01 / 84$ & 353 \\
\hline & & & $11 / 77$ & 206 & & & & $06 / 84$ & 285 \\
\hline & & & $06 / 78$ & 217 & & & & $10 / 84$ & 384 \\
\hline & & & $10 / 78$ & 206 & & & & $01 / 85$ & 343 \\
\hline & & & $01 / 79$ & 242 & & & & $06 / 85$ & 380 \\
\hline & & & $05 / 79$ & 182 & & & & $10 / 85$ & 339 \\
\hline & & & $10 / 80$ & 212 & 1 & & & $01 / 86$ & 266 \\
\hline & & & $02 / 81$ & 215 & & & & $06 / 86$ & 323 \\
\hline & & & $06 / 81$ & 212 & & & & $10 / 86$ & 233 \\
\hline & & & $11 / 81$ & 216 & & & & $01 / 87$ & 252 \\
\hline & & & $06 / 82$ & 194 & & & & $06 / 87$ & 224 \\
\hline & & & $10 / 82$ & 214 & & & & $07 / 87$ & 230 \\
\hline & & & $03 / 83$ & 226 & & & & $01 / 88$ & 187 \\
\hline & & & $06 / 83$ & 215 & & & & $05 / 88$ & 230 \\
\hline & & & $07 / 83$ & 212 & & & & $07 / 88$ & 215 \\
\hline & & & $10 / 83$ & 214 & & & & $11 / 88$ & 213 \\
\hline & & & $01 / 84$ & 210 & $4 \mathrm{~S} / 4 \mathrm{E}-11 \mathrm{Q} 2$ & 468 & $535-948$ & $05 / 73$ & 170 \\
\hline & & & $10 / 84$ & 211 & & & & $09 / 73$ & 149 \\
\hline & & & $01 / 85$ & 225 & & & & $05 / 74$ & 160 \\
\hline & & & $06 / 85$ & 214 & & & & $06 / 74$ & 208 \\
\hline
\end{tabular}


Appendix 5: Dissolved-solids concentration in water from selected wells--Continued

\begin{tabular}{|c|c|c|c|c|c|c|c|c|c|}
\hline $\begin{array}{c}\text { Well } \\
\text { number }\end{array}$ & $\begin{array}{c}\text { Land- } \\
\text { surface } \\
\text { altitude } \\
\text { (feet above } \\
\text { or below (-) } \\
\text { sea level) }\end{array}$ & $\begin{array}{l}\text { Perforated } \\
\text { interval } \\
\text { (depth, in } \\
\text { feet } \\
\text { below land } \\
\text { surface) }\end{array}$ & Date & $\begin{array}{l}\text { Dis- } \\
\text { solved- } \\
\text { solids } \\
\text { concen- } \\
\text { tration } \\
(\mathrm{mg} / \mathrm{L})\end{array}$ & $\begin{array}{c}\text { Well } \\
\text { number }\end{array}$ & $\begin{array}{c}\text { Land- } \\
\text { surface } \\
\text { altitude } \\
\text { (feet above } \\
\text { or below (-) } \\
\text { sea level) }\end{array}$ & $\begin{array}{l}\text { Perforated } \\
\text { interval } \\
\text { (depth, in } \\
\text { feet } \\
\text { below land } \\
\text { surface) }\end{array}$ & Date & $\begin{array}{l}\text { Dis- } \\
\text { solved- } \\
\text { solids } \\
\text { concen- } \\
\text { tration } \\
(\mathrm{mg} / \mathrm{L})\end{array}$ \\
\hline \multirow{22}{*}{\multicolumn{2}{|c|}{ 4S/4E-11Q2-Continued }} & & $09 / 74$ & 192 & \multirow{22}{*}{\multicolumn{2}{|c|}{ 4S/4E-14Q1--Continued }} & & $11 / 81$ & 150 \\
\hline & & & $05 / 75$ & 164 & & & & $02 / 82$ & 194 \\
\hline & & & $05 / 76$ & 148 & & & & $06 / 82$ & 153 \\
\hline & & & $08 / 76$ & 165 & & & & $10 / 82$ & 179 \\
\hline & & & $09 / 76$ & 159 & & & & $02 / 83$ & 176 \\
\hline & & & $05 / 77$ & 143 & & & & $06 / 83$ & 155 \\
\hline & & & $11 / 77$ & 193 & & & & $07 / 83$ & 155 \\
\hline & & & $06 / 78$ & 170 & & & & $10 / 83$ & 173 \\
\hline & & & $01 / 79$ & 164 & & & & $11 / 83$ & 149 \\
\hline & & & $05 / 79$ & 211 & & & & $01 / 84$ & 183 \\
\hline & & & $10 / 80$ & 170 & & & & $06 / 84$ & 193 \\
\hline & & & $02 / 81$ & 164 & & & & $10 / 84$ & 213 \\
\hline & & & $01 / 82$ & 153 & & & & $01 / 85$ & 196 \\
\hline & & & $02 / 83$ & 152 & & & & $06 / 85$ & 171 \\
\hline & & & $01 / 84$ & 149 & & & & $10 / 85$ & 166 \\
\hline & & & $01 / 85$ & 149 & & & & $01 / 86$ & 209 \\
\hline & & & $01 / 86$ & 126 & & & & $06 / 86$ & 198 \\
\hline & & & $01 / 87$ & 147 & & & & $10 / 86$ & 172 \\
\hline & & & $06 / 87$ & 159 & & & & $01 / 87$ & 172 \\
\hline & & & $07 / 87$ & 156 & & & & $06 / 87$ & 177 \\
\hline & & & $01 / 88$ & 128 & & & & $07 / 87$ & 170 \\
\hline & & & $07 / 88$ & 156 & & & & $01 / 88$ & 175 \\
\hline \multirow[t]{5}{*}{$4 S / 4 E-11 R 1$} & 458 & $526-700$ & $09 / 73$ & 145 & & & & $07 / 88$ & 198 \\
\hline & & & $01 / 78$ & 192 & 4S/4E-14R1 & 408 & $560-800$ & $05 / 73$ & 183 \\
\hline & & & $01 / 81$ & 188 & & & & $09 / 73$ & 197 \\
\hline & & & $01 / 84$ & 177 & & & & $07 / 74$ & 270 \\
\hline & & & $01 / 85$ & 182 & & & & $01 / 78$ & 204 \\
\hline \multirow[t]{17}{*}{$4 \mathrm{~S} / 4 \mathrm{E}-13 \mathrm{C} 1$} & 450 & $512-912$ & $07 / 75$ & 183 & & & & $10 / 80$ & 200 \\
\hline & & & $10 / 75$ & 181 & & & & $01 / 81$ & 170 \\
\hline & & & $06 / 78$ & 176 & & & & $06 / 81$ & 194 \\
\hline & & & $10 / 78$ & 170 & & & & $11 / 81$ & 192 \\
\hline & & & $05 / 79$ & 178 & & & & $02 / 82$ & 183 \\
\hline & & & $02 / 81$ & 182 & & & & $06 / 82$ & 194 \\
\hline & & & $06 / 81$ & 182 & & & & $10 / 82$ & 197 \\
\hline & & & $01 / 82$ & 177 & & & & $01 / 83$ & 185 \\
\hline & & & $01 / 84$ & 179 & & & & $06 / 83$ & 155 \\
\hline & & & $01 / 85$ & 178 & & & & $07 / 83$ & 155 \\
\hline & & & $06 / 85$ & 196 & & & & $10 / 83$ & 149 \\
\hline & & & $07 / 85$ & 241 & & & & $01 / 84$ & 147 \\
\hline & & & $01 / 86$ & 153 & & & & $06 / 84$ & 176 \\
\hline & & & $06 / 87$ & 160 & & & & $10 / 84$ & 192 \\
\hline & & & $07 / 87$ & 151 & & & & $01 / 85$ & 180 \\
\hline & & & $01 / 88$ & 142 & & & & $06 / 85$ & 205 \\
\hline & & & $07 / 88$ & 163 & & & & $10 / 85$ & 214 \\
\hline \multirow[t]{3}{*}{ 4S/4E-13Q1 } & 400 & & $01 / 88$ & 314 & & & & $01 / 86$ & 116 \\
\hline & & & $05 / 88$ & 342 & & & & $06 / 86$ & 241 \\
\hline & & & $07 / 88$ & 313 & & & & $10 / 86$ & 221 \\
\hline \multirow[t]{11}{*}{$4 \mathrm{~S} / 4 \mathrm{E}-14 \mathrm{Q} 1$} & 425 & $622-830$ & $11 / 88$ & 275 & & & & $06 / 87$ & 204 \\
\hline & & $850-958$ & $09 / 74$ & 182 & & & & $07 / 87$ & 186 \\
\hline & & & $05 / 75$ & 161 & 4S/4E-23D1 & 435 & $190-408$ & $08 / 74$ & 287 \\
\hline & & & $10 / 75$ & 174 & & & & $02 / 79$ & 104 \\
\hline & & & $11 / 77$ & 178 & & & & $02 / 81$ & 104 \\
\hline & & & $01 / 78$ & 201 & & & & $11 / 81$ & 111 \\
\hline & & & $06 / 78$ & 158 & & & & $01 / 82$ & 106 \\
\hline & & & $11 / 78$ & 198 & & & & $01 / 84$ & 113 \\
\hline & & & $10 / 80$ & 196 & & & & $01 / 85$ & 95 \\
\hline & & & $01 / 81$ & 170 & & & & $06 / 87$ & 210 \\
\hline & & & $06 / 81$ & 154 & & & & $07 / 87$ & 245 \\
\hline
\end{tabular}


Appendix 5: Dissolved-solids concentration in water from selected wells--Continued

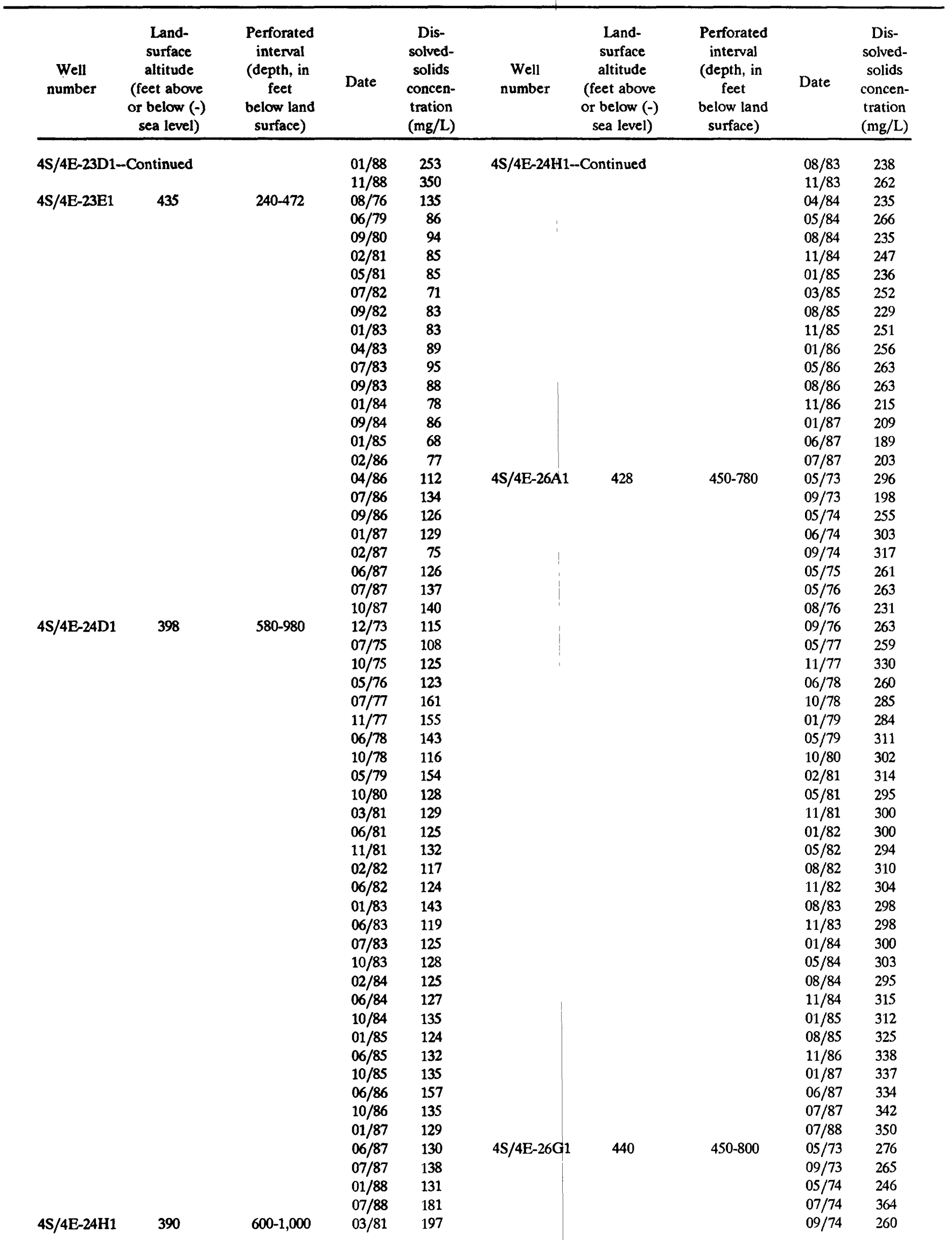

76 Evaluation of a Ground-Water Flow and Transport Model in the Upper Coachella Valley, California 
Appendix 5: Dissolved-solids concentration in water from selected wells--Continued

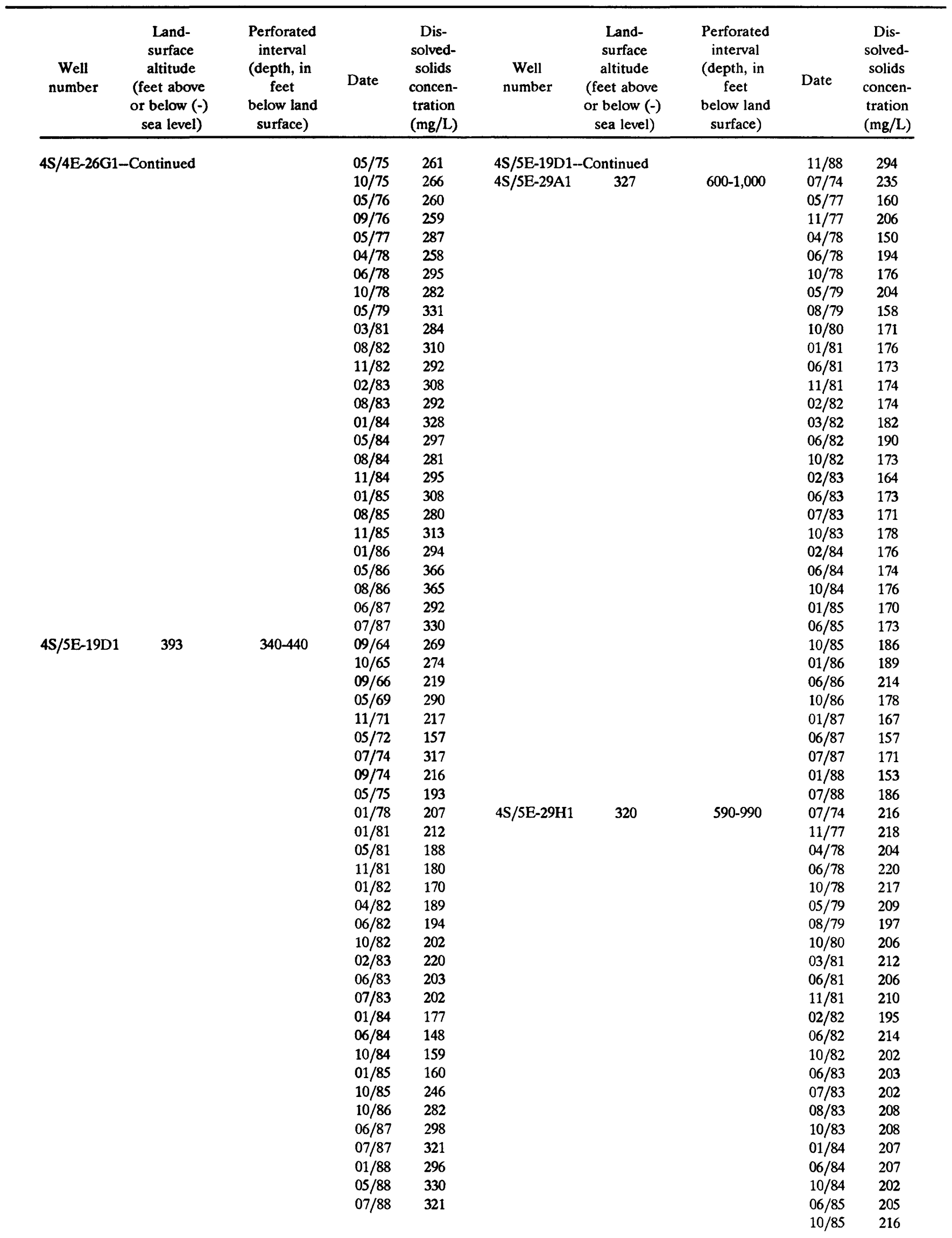


Appendix 5: Dissolved-solids concentration in water from selected wells--Continued

\begin{tabular}{|c|c|c|c|c|c|c|c|c|c|}
\hline $\begin{array}{c}\text { Well } \\
\text { number }\end{array}$ & $\begin{array}{c}\text { Land- } \\
\text { surface } \\
\text { altitude } \\
\text { (feet above } \\
\text { or below }(-) \\
\text { sea level) }\end{array}$ & $\begin{array}{c}\text { Perforated } \\
\text { interval } \\
\text { (depth, in } \\
\text { feet } \\
\text { below land } \\
\text { surface) }\end{array}$ & Date & $\begin{array}{c}\text { Dis- } \\
\text { solved- } \\
\text { solids } \\
\text { concen- } \\
\text { tration } \\
(\mathrm{mg} / \mathrm{L}) \\
\end{array}$ & $\begin{array}{c}\text { Well } \\
\text { number }\end{array}$ & $\begin{array}{c}\text { Land- } \\
\text { surface } \\
\text { altitude } \\
\text { (feet above } \\
\text { or below (-) } \\
\text { sea level) }\end{array}$ & $\begin{array}{l}\text { Perforated } \\
\text { interval } \\
\text { (depth, in } \\
\text { feet } \\
\text { below land } \\
\text { surface) }\end{array}$ & Date & $\begin{array}{c}\text { Dis- } \\
\text { solved- } \\
\text { solids } \\
\text { concen- } \\
\text { tration } \\
(\mathrm{mg} / \mathrm{L})\end{array}$ \\
\hline \multirow{8}{*}{\multicolumn{2}{|c|}{ 4S/5E-29H1--Continued }} & & $01 / 86$ & 215 & \multirow{25}{*}{\multicolumn{2}{|c|}{ 4S/5E-33B4--Continued }} & & $04 / 82$ & 264 \\
\hline & & & $06 / 86$ & 247 & & & & $06 / 82$ & 273 \\
\hline & & & $10 / 86$ & 215 & & & & $08 / 82$ & 250 \\
\hline & & & $01 / 87$ & 221 & & & & $10 / 82$ & 244 \\
\hline & & & $06 / 87$ & 210 & & & & $02 / 83$ & 248 \\
\hline & & & $07 / 87$ & 208 & & & & $04 / 83$ & 244 \\
\hline & & & $01 / 88$ & 163 & & & & $08 / 83$ & 268 \\
\hline & & & $07 / 88$ & 222 & & & & $10 / 83$ & 268 \\
\hline \multirow[t]{4}{*}{ 4S/5E-30C1 } & 357 & $300-440$ & $01 / 88$ & 253 & & & & $01 / 84$ & 224 \\
\hline & & & $05 / 88$ & 294 & & & & $04 / 84$ & 265 \\
\hline & & & $07 / 88$ & 208 & & & & $08 / 84$ & 272 \\
\hline & & & $11 / 88$ & 269 & & & & $10 / 84$ & 288 \\
\hline \multirow[t]{17}{*}{ 4S/5E-33B4 } & 291 & $429-843$ & $05 / 73$ & 274 & & & & $01 / 85$ & 266 \\
\hline & & & $09 / 73$ & 252 & & & & $08 / 85$ & 263 \\
\hline & & & $05 / 74$ & 250 & & & & $10 / 85$ & 305 \\
\hline & & & $06 / 74$ & 268 & & & & $01 / 86$ & 282 \\
\hline & & & $11 / 74$ & 270 & & & & $04 / 86$ & 279 \\
\hline & & & $05 / 77$ & 262 & & & & $08 / 86$ & 355 \\
\hline & & & $07 / 77$ & 279 & & & & $10 / 86$ & 287 \\
\hline & & & $11 / 77$ & 287 & & & & $01 / 87$ & 277 \\
\hline & & & $06 / 78$ & 246 & & & & $06 / 87$ & 282 \\
\hline & & & $10 / 78$ & 172 & & & & $07 / 87$ & 286 \\
\hline & & & $05 / 79$ & 290 & & & & $01 / 88$ & 262 \\
\hline & & & $08 / 79$ & 262 & & & & $07 / 88$ & 322 \\
\hline & & & $06 / 80$ & 269 & & & & $11 / 88$ & 296 \\
\hline & & & $02 / 81$ & 273 & $4 \mathrm{~S} / 5 \mathrm{E}-33 \mathrm{G} 1$ & 294 & $172-436$ & $01 / 88$ & 320 \\
\hline & & & $06 / 81$ & 266 & & & & $05 / 88$ & 358 \\
\hline & & & $11 / 81$ & 252 & & & & $07 / 88$ & 345 \\
\hline & & & $01 / 82$ & 250 & & & & $11 / 88$ & 342 \\
\hline
\end{tabular}

78 Evaluation of a Ground-Water Flow and Transport Model in the Upper Coachella Valley, California 
As written, SUTRA computes a specific storage term as a function of its components: Matrix compressibility and porosity and fluid compressibility (see Voss, 1984, eq. 2.18). Because matrix compressibility is not allowed to vary spatially in the model code, it is not possible to simulate spatially varying aquifer-storage properties. To address this limitation, and to allow direct input of aquifer-storage coefficients for areal models, SUTRA was modified to allow an aquifer-storage coefficient to be read in for each node. Once read in, the storage coefficient is converted to the specific storage term used by SUTRA by dividing the storage coefficient by the aquifer thickness at that node. The simple modifications to the SUTRA code are described below:

\section{Input Data Set 4}

The dummy variable JSTOR is read in in I5 format. The revised data set 4 is:

\begin{tabular}{ll} 
VARIABLE & FORMAT \\
\hline IUNSAT & I5 \\
ISSFLO & I5 \\
ISSTRA & I5 \\
IREAD & I5 \\
ISTOR & I5 \\
JSTOR & I5
\end{tabular}

JSTOR is set to 1 to read in SOP for every node. It is to be 0 otherwise.

\section{Input Data Set $14 \mathrm{~A}$}

The variable STORFAC, a scale factor for the storage coefficient, is read in in G10.0 format. The revised data set $14 \mathrm{~A}$ is:

\begin{tabular}{ll} 
VARIABLE & FORMAT \\
\hline & $5 X$ \\
SCALX & G10.0 \\
SCALY & G10.0 \\
SCALTH & G10.0 \\
PORFAC & G10.0 \\
STORFAC & G10.0
\end{tabular}

\section{Input Data Set 14B}

The variable SOP (II), scaled aquifer storage coefficient is read in in G10.0 format for each of NN nodes. The revised data set $14 \mathrm{~B}$ is:

\begin{tabular}{|c|c|}
\hline VARIABLE & FORMAT \\
\hline II & I5 \\
\hline $\mathrm{X}(\mathrm{II})$ & G10.0 \\
\hline Y(II) & G10.0 \\
\hline THICK(II) & G10.0 \\
\hline POR(II) & G10.0 \\
\hline SOP(II) & G10.0 \\
\hline
\end{tabular}


Appendix 6: Modifications to SUTRA to allow input of spatially varying aquifer-storage coefficient-Continued

The modifications to the following three subroutines are transparent in that a standard input file can be read in and the modifications will be bypassed (JSTOR, STORFAC, and SOP (II) are all taken to be 0). Conversely, a modified input file can be run with the original code (values for the three new variables will not be read in).

These modifications apply when SUTRA is used to simulate areal flow and transport problems. Values for fluid density, viscosity, gravity vector components, and the fluid coefficient of density change with concentration and must be set to the values shown in the example of Rocky Mountain Arsenal study area in Denver, Colorado (Voss, 1984, p. 192), to obtain results in terms of hydraulic head.

$\underline{\text { Subroutine V12842d }}$

--Line A881 replaces A880. JSTOR is included in the common block CONTRL:

COMMON/CONTRL/ GNU,UP,DTMULT,DTMAX,ME,ISSFLO,ISSTRA,ITCYC,

1 NPCYC,NUCYC,NPRINT,IREAD,ISTORE,NOUMAT,IUNSAT,JSTOR

--Line A1871 replaces A1870. JSTOR is read in. If JSTOR=1, SOP will be read in for each node. If not, SUTRA operates in the normal manner:

READ(5,200) IUNSAT,ISSFLO,ISSTRA,IREAD,ISTORE,JSTOR

\section{Subroutine Sutra2d}

--Line B251 replaces B250. JSTOR is included in common block CONTRL:

COMMON/CONTRL/ GNU,UP,DTMULT,DTMAX,ME,ISSFLO,ISSTRA,ITCYC,

1 NPCYC,NUCYC,NPRINT,IREAD,ISTORE,NOUMAT,IUNSAT,JSTOR

B240...

Subroutine Indat 122d

--Line C191 replaces C190. JSTOR is included in common block CONTRL.

COMMON/CONTRL/ GNU,UP,DTMULT,DTMAX,ME,ISSFLO,ISSTRA,ITCYC,

1 NPCYC,NUCYC,NPRINT,IREAD,ISTORE,NOUMAT,IUNSAT,JSTOR

C180...

C191...

--Lines C2641 and C2651 replace C2640 and 2650. STORFAC is read in along with the other nodal scaling factors

(Data set 14A).

C.....INPUT DATASETS 14A AND 14B: NODEWISE DATA

$\mathrm{C} 2630 \ldots$

READ $(5,330)$ SCALX,SCALY,SCALTH,PORFAC,STORFAC

C2641...

330 FORMAT(5X,5G10.0)

C2651...

- Lines C2661, C2671, and C2681 replace C2660, C2670, and C2680.

SOP (II) is read in for each node II along with the other nodal parameters (Data set 14B).

DO $455 \mathrm{I}=1, \mathrm{NN}$

$\mathrm{C} 2661 \ldots$

READ(5,400) II,X(II),Y(II), THICK(II),POR(II),SOP(II)

$\mathrm{C} 2671 \ldots$ 400 FORMAT(I5,5G10.0) 
Appendix 6: Modifications to SUTRA to allow input of spatially varying aquifer-storage coefficient-Continued

-Lines C2731 to C2735 and Line C2741 are inserted.

-If JSTOR $=1$, storage coefficient is converted to specific storage by dividing by thickness and multiplying by STORFAC. Otherwise the read-in value of SOP is ignored and SOP is computed as a function of porosity and fluid and matrix compressibility.

C SET SPECIFIC PRESSURE STORATIVITY, SOP.

$\mathrm{C} 2730 \ldots$

IF(JSTOR.NE.1) GO TO 450

$\mathrm{C} 2731 \ldots$

$\begin{array}{llr}\text { C } \quad \text { COMPUTE SPECIFIC STORAGE FROM AQUIFER STORAGE COEFF. } & \text { C2732... } \\ \text { SOP(II })=\text { SOP(II }) / \text { THICK(II) } & \text { C2733 } \ldots\end{array}$

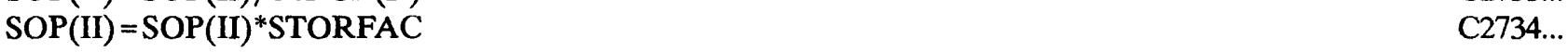

GO TO $455 \quad$ C2735...

450 SOP(II) $=(1 . D 0-P O R(I I))^{*}$ COMPMA + POR(II) ${ }^{*}$ COMPFL $\quad$ C2740...

455 CONTINUE

$\mathrm{C} 2741 \ldots$

-Lines C2751, C2791, C2801, C2811, C2812, C2831, and C2841 replace C2750, C2790, C2800, C2810, C2830, and C2840.

--Scale factor (STORFAC) and nodal values for storage coefficient (SOP) are printed out along with the other nodal parameters.

460 IF(KNODAL.EQ.0) WRITE(6,469) SCALX,SCALY,SCALTH,PORFAC,STORFAC

$\mathrm{C} 2751 \ldots$

469 FORMAT(1H1////11X,' N O D E I N F O R M A T I O N' //16X,

$\mathrm{C} 2760 \ldots$

1 ' PRINTOUT OF NODE COORDINATES, THICKNESSES AND POROSITIES ',

$\mathrm{C} 2770 \ldots$

' CANCELED.' //16X,' SCALE FACTORS :' /33X,1PD15.4,5X,' X-SCALE' /

$\mathrm{C} 2780 \ldots$

1 33X,1PD15.4,5X,' Y-SCALE' /33X,1PD15.4,5X,' THICKNESS FACTOR' /

$\mathrm{C} 2790 \ldots$

33X,1PD15.4,5X,' POROSITY FACTOR' /33X,1PD15.4,5X,

$\mathrm{C} 2791 \ldots$

3 ' STORAGE FACTOR' )

C2801...

IF(KNODAL.EQ. + 1) WRITE(6,470) (I,X(I),Y(I),THICK(I),POR(I),SOP(I),

C2811...

$1 \mathrm{I}=1, \mathrm{NN})$

C2812...

470 FORMAT(1H1//11X,' N O D E I N F O R M A T I O N' //13X,

$\mathrm{C} 2820 \ldots$

1 'NODE' ,7X,' $\mathrm{X}^{\prime}, 16 \mathrm{X}$,' Y' ,17X,' THICKNESS' ,6X,' POROSITY' ,10X,' SOP', C2831...

$2 / /(11 X, 16,3(3 \mathrm{X}, 1 \mathrm{PD} 14.5), 6 \mathrm{X}, 0 \mathrm{PF} 8.5), 3 \mathrm{X}, 1 \mathrm{PD} 14.5)$

C2841... 
Appendix 7: Specified-head values used for southeast boundary of model

[Heads in feet above sea level]

\begin{tabular}{llrlrrrrrr}
\hline \multicolumn{1}{l}{$\begin{array}{l}\text { Model } \\
\text { node }\end{array}$} \\
\cline { 2 - 9 }
\end{tabular}


Appendix 8: SUTRA unit 55 input file for simulation shown in figures 14-16, 18, and 19

[See Voss (1984) for explanation of SUTRA input format]

$7.3092485325546 \mathrm{D}+02$

$9.1414202838240 \mathrm{D}+02$ $1.0280367664628 \mathrm{D}+03$ $6.7551634832975 \mathrm{D}+02$ $6.8236787299960 \mathrm{D}+02$ $8.6644150841152 \mathrm{D}+02$ $5.9637445003192 \mathrm{D}+02$ $1.0312493844393 \mathrm{D}+03$ $5.6534177715512 \mathrm{D}+02$ $9.9593743165737 \mathrm{D}+02$ $5.4566659125671 \mathrm{D}+02$ $9.1833117613636 \mathrm{D}+02$ $5.2774788420714 \mathrm{D}+02$ $8.5134581066725 \mathrm{D}+02$ $5.0128811115803 \mathrm{D}+02$ $8.3465026835037 \mathrm{D}+02$ 4.4968207470205D + 02 $4.5598632116355 \mathrm{D}+02$ $8.4396565008153 \mathrm{D}+02$ 4.1859699001020D + 02 $7.8548933338340 \mathrm{D}+02$ $3.7387415953172 \mathrm{D}+02$ $7.1808530705512 \mathrm{D}+02$ $3.2517824360614 \mathrm{D}+02$ $6.2726915866372 \mathrm{D}+02$ $2.9695600682998 \mathrm{D}+02$ $6.0129662453105 \mathrm{D}+02$ $2.5333316720213 \mathrm{D}+02$ $5.2887000808955 \mathrm{D}+02$ $2.2598315210225 \mathrm{D}+02$ $2.0569554437494 \mathrm{D}+02$ $4.6996256369580 \mathrm{D}+02$ $1.7558462703218 \mathrm{D}+02$ $1.8591504748528 \mathrm{D}+02$ $2.0946709273917 \mathrm{D}+02$ $1.5662605956894 \mathrm{D}+02$ $1.5739247388343 \mathrm{D}+02$ $3.5476363889643 \mathrm{D}+02$ $1.4051783151041 \mathrm{D}+02$ $1.3456818721185 \mathrm{D}+02$ $1.3552493643174 \mathrm{D}+02$ $1.1726918808057 \mathrm{D}+02$ $1.1454219934325 \mathrm{D}+02$ $1.5971412324223 \mathrm{D}+02$ $9.3823126850431 \mathrm{D}+01$ $9.3105357632458 \mathrm{D}+01$ $6.2588260713489 \mathrm{D}+01$ $7.7002283542440 \mathrm{D}+01$ $6.4310503682001 \mathrm{D}+01$ $5.6283464010878 \mathrm{D}+01$ $4.8175855890521 \mathrm{D}+01$ $4.7299956508256 \mathrm{D}+01$ $5.5551462324414 \mathrm{D}+01$
$7.0985899300306 \mathrm{D}+02$ $7.5135094740934 \mathrm{D}+02$ $1.0309778003080 \mathrm{D}+03$ $6.5822353333552 \mathrm{D}+02$ $1.0242472371306 \mathrm{D}+03$ $6.7785048055671 \mathrm{D}+02$ $6.0827924715137 \mathrm{D}+02$ $1.0261322763394 \mathrm{D}+03$ $5.5870359083219 \mathrm{D}+02$ $1.0585613610923 \mathrm{D}+03$ $5.4400946289647 \mathrm{D}+02$ $9.4151969915621 \mathrm{D}+02$ $5.2443561250320 \mathrm{D}+02$ $8.6177690499184 \mathrm{D}+02$ $4.9926721345866 \mathrm{D}+02$ $8.4987093124908 \mathrm{D}+02$ $4.5598837068753 \mathrm{D}+02$ $4.5236051598971 \mathrm{D}+02$ 4.3671469692823D +02 4.1228747295351D + 02 $7.8436902327703 \mathrm{D}+02$ $3.7165153368524 \mathrm{D}+02$ $6.9157991905751 \mathrm{D}+02$ $3.3137274015248 \mathrm{D}+02$ $6.1499238897673 \mathrm{D}+02$ $2.9389089520781 \mathrm{D}+02$ $5.9714688841844 \mathrm{D}+02$ $2.5190601202937 \mathrm{D}+02$ $5.2784711098947 \mathrm{D}+02$ $2.0905805549056 \mathrm{D}+02$ $2.0424672044664 \mathrm{D}+02$ $1.6924167316439 \mathrm{D}+02$ $1.7965634458356 \mathrm{D}+02$ $4.0026843083948 \mathrm{D}+02$ $1.5034019854309 \mathrm{D}+02$ $1.5764687493828 \mathrm{D}+02$ $1.5631070736762 \mathrm{D}+02$ $3.6802803644395 \mathrm{D}+02$ $1.3510790274199 \mathrm{D}+02$ $1.3486544112832 \mathrm{D}+02$ $1.6422256848469 \mathrm{D}+02$ $1.1687151675557 \mathrm{D}+02$ 1.1632941240120D + 02 $1.9356574470382 \mathrm{D}+02$ $9.2697072592709 \mathrm{D}+01$ $9.7259199037020 \mathrm{D}+01$ $6.2706484423383 \mathrm{D}+01$ $7.9136048812587 \mathrm{D}+01$ $6.9567042477375 \mathrm{D}+01$ $6.1350950845334 \mathrm{D}+01$ $4.8164279136752 \mathrm{D}+01$ $4.6958785034038 \mathrm{D}+01$ $5.9020202930374 \mathrm{D}+01$
$7.5947662928802 \mathrm{D}+02$ $7.2642196442595 \mathrm{D}+02$ $7.9447895312718 \mathrm{D}+02$ $6.6485228566834 \mathrm{D}+02$ $1.0298200956512 \mathrm{D}+03$ $6.2023543530216 \mathrm{D}+02$ $6.2560919271569 \mathrm{D}+02$ $6.3387464504295 \mathrm{D}+02$ $5.5598494955718 \mathrm{D}+02$ $9.8347073323853 \mathrm{D}+02$ $5.4030695526537 \mathrm{D}+02$ $9.7160375021260 \mathrm{D}+02$ $5.2126415252395 \mathrm{D}+02$ $8.7578455283921 \mathrm{D}+02$ $4.9786948363961 \mathrm{D}+02$ $4.7168548249930 \mathrm{D}+02$ 4.5840329767371D + 02 8.1284513319495D + 02 $4.1258028188337 \mathrm{D}+02$ $4.0838635537657 \mathrm{D}+02$ $3.7290844534780 \mathrm{D}+02$ $3.7041036467841 \mathrm{D}+02$ $3.2725652825799 \mathrm{D}+02$ 3.3263903435967D + 02 $2.9693965610796 \mathrm{D}+02$ $2.9307271026353 \mathrm{D}+02$ $2.6707917726512 \mathrm{D}+02$ $2.4805023346848 \mathrm{D}+02$ $5.1569297350584 \mathrm{D}+02$ $2.0422328066654 \mathrm{D}+02$ $4.4424687660599 \mathrm{D}+02$ $1.7888488684181 \mathrm{D}+02$ $1.8407410595728 \mathrm{D}+02$ 4. $1660831755988 \mathrm{D}+02$ $1.5263296360193 \mathrm{D}+02$ $1.5609186980912 \mathrm{D}+02$ $1.5468066032123 \mathrm{D}+02$ $2.2549846109497 \mathrm{D}+02$ $1.4388153411659 \mathrm{D}+02$ $1.3631637226744 \mathrm{D}+02$ $2.5145618769252 \mathrm{D}+02$ $1.1802690526583 \mathrm{D}+02$ $1.1822336661968 \mathrm{D}+02$ $9.6373129136533 \mathrm{D}+01$ $9.3326114969678 \mathrm{D}+01$ $1.1112029932173 \mathrm{D}+02$ 6.5348342324987D + 01 $8.1875495589795 \mathrm{D}+01$ $5.1601559873885 \mathrm{D}+01$ $6.2527580116289 \mathrm{D}+01$ 4.3664821992153D + 01 $5.5160921541586 \mathrm{D}+01$ $6.1036384994262 \mathrm{D}+01$
$9.0355513146015 \mathrm{D}+02$ $1.0215110599278 \mathrm{D}+03$ $7.0988839112659 \mathrm{D}+02$ $6.8586759783335 \mathrm{D}+02$ $1.0404959569667 \mathrm{D}+03$ $6.0008339113558 \mathrm{D}+02$ $1.0279101909645 \mathrm{D}+03$ $5.8030887660840 \mathrm{D}+02$ $5.6366801332397 \mathrm{D}+02$ $5.5502119933749 \mathrm{D}+02$ $5.4172629843688 \mathrm{D}+02$ $5.4254000363596 \mathrm{D}+02$ $5.1908197561392 \mathrm{D}+02$ $4.9668735466222 \mathrm{D}+02$ $8.3065185155742 \mathrm{D}+02$ $4.6095537366492 \mathrm{D}+02$ $4.5955956093108 \mathrm{D}+02$ $8.1634947682564 \mathrm{D}+02$ 4.1414677652447D + 02 $7.8995408021504 \mathrm{D}+02$ $3.7439875016622 \mathrm{D}+02$ $7.1908339747009 \mathrm{D}+02$ 3.2404685057230D + 02 $6.3120746158595 \mathrm{D}+02$ $2.9860571436783 \mathrm{D}+02$ $6.0273638425061 \mathrm{D}+02$ $2.6681492177270 \mathrm{D}+02$ $2.4545321966019 \mathrm{D}+02$ $2.0628712346115 \mathrm{D}+02$ $2.0454508798768 \mathrm{D}+02$ $4.6342531173271 \mathrm{D}+02$ $1.6890318373982 \mathrm{D}+02$ $1.8667273624421 \mathrm{D}+02$ $4.3016815598030 \mathrm{D}+02$ $1.6004991723578 \mathrm{D}+02$ $1.5757591866173 \mathrm{D}+02$ $2.8355381497802 \mathrm{D}+02$ $1.5775197289566 \mathrm{D}+02$ $1.3992665768102 \mathrm{D}+02$ $1.3609116421867 \mathrm{D}+02$ $2.8247787219170 \mathrm{D}+02$ $1.1582481859778 \mathrm{D}+02$ $1.3771874236294 \mathrm{D}+02$ $9.5767139413962 \mathrm{D}+01$ $9.3267348204016 \mathrm{D}+01$ $9.9337026562304 \mathrm{D}+01$ $7.1783671575515 \mathrm{D}+01$ $7.0182175441146 \mathrm{D}+01$ $5.3532558759397 \mathrm{D}+01$ 6.5922968627814D + 01 $4.7808882127584 \mathrm{D}+01$ $5.6456767669702 \mathrm{D}+01$ $4.6323080604772 \mathrm{D}+01$ 
Appendix 8: SUTRA unit 55 input file for simulation shown in figures 14-16, 18, and 19--Continued

\subsection{D+01 $4.0994262554444 \mathrm{D}+01$ $4.8862272077292 \mathrm{D}+01$ $3.6085420260682 \mathrm{D}+01$ $3.7353584218654 \mathrm{D}+01$ $3.4072086299846 \mathrm{D}+01$ $2.5177194246082 \mathrm{D}+01$ $2.4713197997854 \mathrm{D}+01$ $1.7003914233003 \mathrm{D}+01$ $2.2633467346254 \mathrm{D}+01$ $4.0282847083805 \mathrm{D}+00$} 200.

200.

200.

200.

200.

200.

200.

200.

200.

200.

200.

200.

200.

200.

200.

200.

200.

200.

200.

200.

200.

200.

200.

200.

200.

200.

200.

200.

200.

200.

200.

200.

200.

200.

200.

200.

200.

200.

200.

200.

200.
$4.0634621667843 \mathrm{D}+01$

4.3664400429114D+01

$5.1247760449877 \mathrm{D}+01$

$2.8744393940544 \mathrm{D}+01$

$3.6146514810369 \mathrm{D}+01$

$2.7044969876938 \mathrm{D}+01$

$2.8157292686757 \mathrm{D}+01$

$2.0765027493446 \mathrm{D}+01$

$1.6329511627487 \mathrm{D}+01$

$3.1963439423736 \mathrm{D}+01$

$-7.8933466533485 \mathrm{D}+00$

200.

200.

200.

200.

200.

200.

200.

200.

200.

200.

200.

200.

200.

200.

200.

200.

200.

200.

200.

200.

200.

200.

200.

200.

200.

200.

200.

200.

200.

200.

200.

200.

200.

200.

200.

200.

200.

200.

200.

200.

200.
3.6941064839752D + 01

$4.4608203720932 \mathrm{D}+01$

$4.5906821042814 \mathrm{D}+01$

$2.8235776955295 \mathrm{D}+01$

$3.6352799272966 \mathrm{D}+01$

$2.4487171085308 \mathrm{D}+01$

$2.9452373376748 \mathrm{D}+01$

$2.2528679746854 \mathrm{D}+01$

$7.6492221299874 \mathrm{D}+00$

$2.0990636710470 \mathrm{D}+01$

$-2.0270212367722 \mathrm{D}+00$

200.

200.

200.

200.

200.

200.

200.

200.

200.

200.

200.

200.

200.

200.

200.

200.

200.

200.

200.

200.

200.

200.

200.

200.

200.

200.

200.

200.

200.

200.

200.

200.

200.

200.

200.

200.

200.

200.

200.

200.

200.
$3.7265755189505 \mathrm{D}+01$ $4.5552149115976 \mathrm{D}+01$ 4.4460649518241D + 01 3.3414227316017D + 01 3.2311447235571D + 01 $2.4131098019584 \mathrm{D}+01$ $2.1930580048160 \mathrm{D}+01$ $2.0753084551694 \mathrm{D}+01$ $-9.1682785810257 \mathrm{D}-01$ $9.0252694465452 \mathrm{D}+00$

200.

200.

200.

200.

200.

200.

200.

200.

200.

200.

200.

200.

200.

200.

200.

200.

200.

200.

200.

200.

200.

200.

200.

200.

200.

200.

200.

200.

200.

200.

200.

200.

200.

200.

200.

200.

200.

200.

200.

200.

200. 
Appendix 8: SUTRA unit 55 input file for simulation shown in figures 14-16, 18, and 19--Continued

\begin{tabular}{llll}
\hline 200. & 200. & 200. & 200. \\
200. & 200. & 200. & 200. \\
200. & 200. & 200. & 200. \\
200. & 200. & 200. & 200. \\
200. & 200. & 200. & 200. \\
200. & 200. & 200. & 200. \\
200. & 200. & 200. & 200. \\
200. & 200. & 200. & 200. \\
200. & 200. & 200. & 200. \\
200. & 200. & 200. & 200. \\
200. & 200. & 200. & 200. \\
200. & 200. & 200. & 200. \\
200. & 200. & 200. & 200. \\
200. & 200. & 200. & 200. \\
200. & 200. & 200. & 200. \\
200. & 200. & 200. & 200. \\
200. & 200. & 200. & 200. \\
200. & 200. & 200. & 200. \\
200. & 200. & 200. & 200. \\
200. & 200. & 200. & 200. \\
200. & 200. & 200. & 200. \\
200. & 200. & 200. & \\
200. & 200. & 200. & \\
200. & 200. & & \\
\hline
\end{tabular}

Appendixes 85 
[See Voss, 1984, and appendix 6 for explanation of SUTRA input format]

SUTRA SOLUTE TRANSPORT coachella add $350 \mathrm{ft}$ thick in AR area COACHELLA VALLEY --1979-86 verif. runs .65 RETURN, QUARTERLY STRESS CHANGES 10 TIME STEPS/QUARTER, set $s$ to .15 in PS region, .1 IN plm CAN.,

\begin{tabular}{|c|c|c|c|c|c|c|c|c|c|}
\hline 255 & 209 & 40 & 0 & 7 & 00 & 127 & 0 & 9 & 99 \\
\hline 0 & +0 & +0 & +01 & +01 & 1 & & & & \\
\hline 0.00000 & $1.00 \mathrm{E}+02$ & & & & & & & & \\
\hline 320 & 7.88940E05 & \multicolumn{2}{|c|}{$2.524608 E+08$} & 1 & 1.0 & \multicolumn{2}{|c|}{$3.15576+10$} & 1 & 01 \\
\hline 319 & 1 & 0 & 0 & +1 & +1 & 001 & 1 & & \\
\hline 01 & & & & & & & & & \\
\hline $0.000 \mathrm{E} 00$ & 0001.0 & 0.0 & $1.0 \mathrm{E} 00$ & 0.00 & 0.00 & 1.00 & & & \\
\hline $0.0 \mathrm{E} 0$ & $0.0 \mathrm{E} 0$ & $0.0 \mathrm{E} 0$ & 0001.0 & & & & & & \\
\hline NONE & & & & & & & & & \\
\hline $0.0 \mathrm{E}-9$ & $0.0 \mathrm{E}-9$ & $0.0 \mathrm{E}-9$ & $0.0 \mathrm{E}-9$ & & & & & & \\
\hline 00.0 & 00.0 & 1.0 & & & & & & & \\
\hline NODE & 100.0 & 100.0 & 1.00 & 1. & 1. & & & & \\
\hline 1 & 142. & 470. & 700. & 0.250 & 0.130 & & & & \\
\hline 2 & 137. & 499. & 725. & 0.250 & 0.130 & & & & \\
\hline 3 & 110. & 538. & 750. & 0.250 & 0.130 & & & & \\
\hline 4 & 79.0 & 558. & 750. & 0.250 & 0.130 & & & & \\
\hline 5 & 66.0 & 582. & 725. & 0.250 & 0.130 & & & & \\
\hline 6 & 100. & 635. & 600. & 0.250 & 0.130 & & & & \\
\hline 7 & 110. & 645. & 400 & 0.250 & 0.130 & & & & \\
\hline 8 & 115. & 663. & 250. & 0.250 & 0.140 & & & & \\
\hline 9 & 124. & 683. & 200. & 0.250 & 0.150 & & & & \\
\hline 10 & 129. & 702. & 200. & 0.250 & 0.150 & & & & \\
\hline 11 & 175. & 457. & 700. & 0.250 & 0.130 & & & & \\
\hline 12 & 165. & 500. & 725. & 0.250 & 0.130 & & & & \\
\hline 13 & 156. & 535. & 750. & 0.250 & 0.130 & & & & \\
\hline 14 & 149. & 567. & 750. & 0.250 & 0.130 & & & & \\
\hline 15 & 142. & 602. & 737. & 0.250 & 0.130 & & & & \\
\hline 16 & 134. & 632. & 612. & 0.250 & 0.130 & & & & \\
\hline 17 & 138. & 643. & 400. & 0.250 & 0.130 & & & & \\
\hline 18 & 142. & 661. & 250. & 0.250 & 0.140 & & & & \\
\hline 19 & 150. & 682. & 200. & 0.250 & 0.150 & & & & \\
\hline 20 & 157. & 710. & 200. & 0.250 & 0.150 & & & & \\
\hline 21 & 200. & 452. & 700. & 0.250 & 0.130 & & & & \\
\hline 22 & 193. & 500. & 733. & 0.250 & 0.130 & & & & \\
\hline 23 & 195. & 541. & 1100. & 0.250 & 0.130 & & & & \\
\hline 24 & 196. & 571. & 1100. & 0.250 & 0.130 & & & & \\
\hline 25 & 194. & 597. & 1100. & 0.250 & 0.130 & & & & \\
\hline 26 & 182. & 623. & 1037. & 0.250 & 0.130 & & & & \\
\hline 27 & 172. & 638. & 912. & 0.250 & 0.135 & & & & \\
\hline 28 & 176. & 657. & 425. & 0.250 & 0.145 & & & & \\
\hline 29 & 181. & 682. & 350. & 0.250 & 0.150 & & & & \\
\hline 30 & 190. & 724. & 350. & 0.250 & 0.150 & & & & \\
\hline 31 & 222. & 505. & 750. & 0.250 & 0.130 & & & & \\
\hline 32 & 233. & 541. & 1100. & 0.250 & 0.130 & & & & \\
\hline 33 & 234. & 573. & 1100. & 0.250 & 0.130 & & & & \\
\hline 34 & 237. & 598. & 1100. & 0.250 & 0.130 & & & & \\
\hline 35 & 240. & 621. & 1100. & 0.250 & 0.130 & & & & \\
\hline 36 & 220. & 644. & 1087. & 0.250 & 0.135 & & & & \\
\hline 37 & 213. & 654. & 662. & 0.250 & 0.145 & & & & \\
\hline 38 & 205. & 681. & 600. & 0.250 & 0.150 & & & & \\
\hline
\end{tabular}




\begin{tabular}{|c|c|c|c|c|c|}
\hline 39 & 210. & 727. & 600 & 0.250 & 0.150 \\
\hline 40 & 262. & 552. & 1100. & 0.250 & 0.130 \\
\hline 41 & 263. & 574. & 1100. & 0.250 & 0.130 \\
\hline 42 & 263. & 600. & 1100 & 0.250 & 0.130 \\
\hline 43 & 264. & 623. & 1100. & 0.250 & 0.130 \\
\hline 44 & 264. & 640. & 1062. & 0.250 & 0.130 \\
\hline 45 & 261. & 650. & 637. & 0.250 & 0.140 \\
\hline 46 & 252. & 685. & 650. & 0.250 & 0.150 \\
\hline 47 & 238. & 738. & 700 & 0.250 & 0.150 \\
\hline 48 & 283. & 557. & 1100. & 0.250 & 0.130 \\
\hline 49 & 284. & 576. & 1100. & 0.250 & 0.130 \\
\hline 50 & 286. & 601. & 1112. & 0.250 & 0.130 \\
\hline 51 & 287. & 623. & 1125. & 0.250 & 0.130 \\
\hline 52 & 288. & 642. & 1062. & 0.250 & 0.130 \\
\hline 53 & 288. & 651. & 600 & 0.250 & 0.127 \\
\hline 54 & 288. & 688. & 625. & 0.250 & 0.125 \\
\hline 55 & 290. & 740. & 700 & 0.250 & 0.125 \\
\hline 56 & 308. & 577. & 1117. & 0.250 & 0.130 \\
\hline 57 & 310. & 601. & 1137. & 0.250 & 0.130 \\
\hline 58 & 310. & 623. & 1150. & 0.250 & 0.130 \\
\hline 59 & 311. & 642. & 1100. & 0.250 & 0.130 \\
\hline 60 & 311. & 651. & 662 & 0.250 & 0.115 \\
\hline 61 & 312. & 689. & 675. & 0.250 & 0.100 \\
\hline 62 & 316. & 742. & 725. & 0.250 & 0.100 \\
\hline 63 & 310. & 557. & 1075. & 0.250 & 0.130 \\
\hline 64 & 310. & 530. & 1050. & 0.250 & 0.130 \\
\hline 65 & 342. & 530. & 1050. & 0.250 & 0.150 \\
\hline 66 & 342. & 556. & 1075. & 0.250 & 0.150 \\
\hline 67 & 342. & 577. & 1125. & 0.250 & 0.150 \\
\hline 68 & 343. & 600. & 1150. & 0.250 & 0.150 \\
\hline 69 & 343. & 623. & 1150. & 0.250 & 0.150 \\
\hline 70 & 344. & 643. & 1125. & 0.250 & 0.102 \\
\hline 71 & 344. & 652. & 762. & 0.250 & 0.115 \\
\hline 72 & 343. & 692. & 825. & 0.250 & 0.100 \\
\hline 73 & 343. & 744. & 875. & 0.250 & 0.100 \\
\hline 74 & 375. & 532. & 1050. & 0.250 & 0.150 \\
\hline 75 & 376. & 557. & 1100. & 0.250 & 0.150 \\
\hline 76 & 377. & 578. & 1150 & 0.250 & 0.150 \\
\hline 77 & 378. & 601. & 1175. & 0.250 & 0.150 \\
\hline 78 & 380. & 623. & 1187. & 0.250 & 0.150 \\
\hline 79 & 381. & 647. & 1200 & 0.250 & 0.150 \\
\hline 80 & 381. & 656. & 875 & 0.250 & 0.100 \\
\hline 81 & 385. & 698. & 950. & 0.250 & 0.100 \\
\hline 82 & 387. & 744. & $0.100 \mathrm{E}+04$ & 0.250 & 0.100 \\
\hline 83 & 409. & 558. & 1150. & 0.250 & 0.150 \\
\hline 84 & 411. & 580. & 1175. & 0.250 & 0.150 \\
\hline 85 & 412. & 602. & 1212. & 0.250 & 0.150 \\
\hline 86 & 415. & 627. & 1237. & 0.250 & 0.150 \\
\hline 87 & 417. & 648. & 1250. & 0.250 & 0.150 \\
\hline 88 & 417. & 657. & 912. & 0.250 & 0.100 \\
\hline 89 & 420. & 698. & 962. & 0.250 & 0.100 \\
\hline 90 & 425. & 742. & $0.100 \mathrm{E}+04$ & 0.250 & 0.100 \\
\hline 91 & 444. & 543. & 1150. & 0.250 & 0.150 \\
\hline 92 & 458. & 582. & 1175. & 0.250 & 0.150 \\
\hline 93 & 462. & 608. & 1225. & 0.250 & 0.150 \\
\hline
\end{tabular}




\begin{tabular}{|c|c|c|c|c|c|}
\hline 94 & 457. & 634. & 1262. & 0.250 & 0.150 \\
\hline 95 & 455. & 650. & 1287. & 0.250 & 0.150 \\
\hline 96 & 452. & 658. & 962. & 0.250 & 0.100 \\
\hline 97 & 456. & 694. & 987. & 0.250 & 0.100 \\
\hline 98 & 461. & 744. & $0.100 \mathrm{E}+04$ & 0.250 & 0.100 \\
\hline 99 & 477. & 537. & 1175. & 0.250 & 0.150 \\
\hline 100 & 485. & 581. & 1200. & 0.250 & 0.150 \\
\hline 101 & 490. & 611. & 1250. & 0.250 & 0.150 \\
\hline 102 & 496. & 638. & 1300. & 0.250 & 0.150 \\
\hline 103 & 498. & 652. & 1337. & 0.250 & 0.150 \\
\hline 104 & 499. & 660. & $0.100 \mathrm{E}+04$ & 0.250 & 0.100 \\
\hline 105 & 504. & 702. & $0.100 E+04$ & 0.250 & 0.100 \\
\hline 106 & 508. & 751. & $0.100 \mathrm{E}+04$ & 0.250 & 0.100 \\
\hline 107 & 498. & 492. & 850. & 0.250 & 0.150 \\
\hline 108 & 508. & 520. & 1217. & 0.250 & 0.150 \\
\hline 109 & 548. & 574. & 1250. & 0.250 & 0.150 \\
\hline 110 & 556. & 608. & 1300. & 0.250 & 0.150 \\
\hline 111 & 563. & 640. & 1337. & 0.250 & 0.150 \\
\hline 112 & 566. & 655. & $0.135 E+04$ & 0.250 & 0.150 \\
\hline 113 & 566. & 663. & $0.100 \mathrm{E}+04$ & 0.250 & 0.100 \\
\hline 114 & 571. & 711. & $0.100 E+04$ & 0.250 & 0.100 \\
\hline 115 & 578. & 762. & $0.100 \mathrm{E}+04^{\circ}$ & 0.250 & 0.100 \\
\hline 116 & 570. & 455. & 850. & 0.250 & 0.100 \\
\hline 117 & 557. & 488. & 900. & 0.250 & 0.150 \\
\hline 118 & 594. & 514. & 937. & 0.250 & 0.150 \\
\hline 119 & 613. & 556. & 962. & 0.250 & 0.150 \\
\hline 120 & 621. & 596. & 987. & 0.250 & 0.150 \\
\hline 121 & 628. & 632. & $0.100 \mathrm{E}+04$ & 0.250 & 0.150 \\
\hline 122 & 634. & 660. & $0.100 \mathrm{E}+04$ & 0.250 & 0.150 \\
\hline 123 & 633. & 669. & $0.100 \mathrm{E}+04$ & 0.250 & 0.100 \\
\hline 124 & 633. & 706. & $0.100 \mathrm{E}+04$ & 0.250 & 0.100 \\
\hline 125 & 633. & 757. & $0.100 \mathrm{E}+04$ & 0.250 & 0.100 \\
\hline 126 & 625. & 378. & 900. & 0.350 & 0.100 \\
\hline 127 & 618. & 441. & 917. & 0.317 & 0.100 \\
\hline 128 & 656. & 471. & 962. & 0.300 & 0.150 \\
\hline 129 & 660. & 500. & $0.100 \mathrm{E}+04$ & 0.300 & 0.150 \\
\hline 130 & 664. & 533. & $0.100 \mathrm{E}+04$ & 0.275 & 0.150 \\
\hline 131 & 672. & 584. & $0.100 \mathrm{E}+04$ & 0.250 & 0.150 \\
\hline 132 & 677. & 627. & $0.100 \mathrm{E}+04$ & 0.250 & 0.150 \\
\hline 133 & 685. & 655. & $0.100 \mathrm{E}+04$ & 0.250 & 0.150 \\
\hline 134 & 685. & 663. & $0.100 \mathrm{E}+04$ & 0.250 & 0.100 \\
\hline 135 & 686. & 709. & $0.100 \mathrm{E}+04$ & 0.250 & 0.100 \\
\hline 136 & 675. & 757. & $0.100 \mathrm{E}+04$ & 0.250 & 0.100 \\
\hline 137 & 722. & 216. & 750. & 0.200 & 0.100 \\
\hline 138 & 702. & 267. & 800. & 0.200 & 0.100 \\
\hline 139 & 685. & 315. & 925. & 0.200 & 0.100 \\
\hline 140 & 657. & 368. & 967. & 0.300 & 0.100 \\
\hline 141 & 667. & 416. & 975. & 0.350 & 0.100 \\
\hline 142 & 698. & 446. & $0.100 \mathrm{E}+04$ & 0.350 & 0.150 \\
\hline 143 & 740. & 486. & $0.100 \mathrm{E}+04$ & 0.350 & 0.150 \\
\hline 144 & 750. & 521. & $0.100 \mathrm{E}+04$ & 0.300 & 0.150 \\
\hline 145 & 767. & 573. & $0.100 \mathrm{E}+04$ & 0.250 & 0.150 \\
\hline 146 & 779. & 614. & $0.100 E+04$ & 0.250 & 0.150 \\
\hline 147 & 790. & 643. & $0.100 \mathrm{E}+04$ & 0.250 & 0.150 \\
\hline
\end{tabular}




\begin{tabular}{|c|c|c|c|c|c|}
\hline 148 & 786. & 651. & $0.100 E+04$ & 0.250 & 0.100 \\
\hline 149 & 776. & 710. & $0.100 E+04$ & 0.250 & 0.112 \\
\hline 150 & 770. & 760. & $0.100 E+04$ & 0.250 & 0.125 \\
\hline 151 & 770. & 220. & 750. & 0.200 & 0.100 \\
\hline 152 & 760. & 265. & 800. & 0.200 & 0.100 \\
\hline 153 & 748. & 324. & 925. & 0.200 & 0.100 \\
\hline 154 & 760. & 348. & $0.100 E+04$ & 0.275 & 0.100 \\
\hline 155 & 780. & 390. & 933. & 0.350 & 0.100 \\
\hline 156 & 802. & 417. & 950. & 0.350 & 0.150 \\
\hline 57 & 838. & 466. & $0.100 E+04$ & 0.350 & 0.150 \\
\hline 158 & 851. & 507. & $0.100 E+04$ & 0.300 & 0.150 \\
\hline 159 & 867. & 563. & $0.100 E+04$ & 0.250 & 0.150 \\
\hline 160 & 883. & 622. & $0.100 E+04$ & 0.250 & 0.150 \\
\hline 161 & 888. & 642. & $0.100 E+04$ & 0.250 & 0.150 \\
\hline 162 & 881. & 651. & $0.100 E+04$ & 0.237 & 0.150 \\
\hline 163 & 878. & 704. & $0.100 E+04$ & 0.225 & 0.137 \\
\hline 164 & 874. & 752. & $0.100 E+04$ & 0.225 & 0.150 \\
\hline 165 & 914. & 395. & 850. & 0.350 & 0.150 \\
\hline 166 & 914. & 420. & 925. & 0.350 & 0.150 \\
\hline 167 & 914. & 453. & $0.100 E+04$ & 0.350 & 0.150 \\
\hline 168 & 926. & 500. & $0.100 E+04$ & 0.312 & 0.150 \\
\hline 169 & 941. & 557. & $0.100 \mathrm{E}+04$ & 0.275 & 0.150 \\
\hline 170 & 955. & 612. & $0.100 E+04$ & 0.275 & 0.150 \\
\hline 171 & 964. & 657. & $0.100 E+04$ & 0.250 & 0.150 \\
\hline 172 & 957. & 665. & $0.100 E+04$ & 0.212 & 0.115 \\
\hline 173 & 967. & 700. & $0.100 E+04$ & 0.200 & 0.150 \\
\hline 174 & 965. & 750. & $0.100 \mathrm{E}+04$ & 0.200 & 0.150 \\
\hline 175 & 965. & 392. & 900. & 0.350 & 0.150 \\
\hline 176 & 975. & 417. & 950. & 0.350 & 0.150 \\
\hline 177 & 986. & 437. & $0.100 E+04$ & 0.337 & 0.150 \\
\hline 178 & $0.101 E+04$ & 500. & $0.100 E+04$ & 0.312 & 0.150 \\
\hline 179 & $0.102 E+04$ & 544. & $0.100 E+04$ & 0.300 & 0.150 \\
\hline 180 & $0.103 E+04$ & 606. & $0.100 E+04$ & 0.300 & 0.150 \\
\hline 181 & $0.104 E+04$ & 673. & $0.100 E+04$ & 0.260 & 0.126 \\
\hline 182 & $0.104 E+04$ & 682. & $0.100 E+04$ & 0.200 & 0.120 \\
\hline 183 & $0.103 E+04$ & 706. & $0.100 E+04$ & 0.200 & 0.150 \\
\hline 184 & $0.106 E+04$ & 728. & $0.100 E+04$ & 0.200 & 0.150 \\
\hline 185 & $0.105 E+04$ & 345. & 925. & 0.375 & 0.150 \\
\hline 186 & $0.106 E+04$ & 379. & 962. & 0.375 & 0.150 \\
\hline 187 & $0.107 E+04$ & 427. & $0.100 E+04$ & 0.362 & 0.150 \\
\hline 188 & $0.109 E+04$ & 500. & $0.100 E+04$ & 0.337 & 0.150 \\
\hline 189 & $0.108 E+04$ & 533. & $0.100 E+04$ & 0.312 & 0.150 \\
\hline 190 & $0.108 E+04$ & 595. & $0.100 E+04$ & 0.287 & 0.150 \\
\hline 191 & $0.107 E+04$ & 635. & $0.100 E+04$ & 0.283 & 0.150 \\
\hline 192 & $0.112 E+04$ & 654. & $0.100 E+04$ & 0.287 & 0.170 \\
\hline 193 & $0.115 E+04$ & 714. & $0.100 E+04$ & 0.267 & 0.140 \\
\hline 194 & $0.113 E+04$ & 716. & $0.100 E+04$ & 0.200 & 0.115 \\
\hline 195 & $0.113 E+04$ & 327. & 975. & 0.400 & 0.170 \\
\hline 196 & $0.114 E+04$ & 372. & 987. & 0.400 & 0.170 \\
\hline 197 & $0.116 E+04$ & 432. & $0.100 E+04$ & 0.387 & 0.170 \\
\hline 198 & $0.115 E+04$ & 498. & $0.100 E+04$ & 0.362 & 0.170 \\
\hline 199 & $0.115 E+04$ & 536. & $0.100 E+04$ & 0.325 & 0.170 \\
\hline 200 & $0.114 E+04$ & 582. & $0.100 E+04$ & 0.287 & 0.170 \\
\hline 201 & $0.124 E+04$ & 164. & 750. & 0.200 & 0.190 \\
\hline
\end{tabular}




\begin{tabular}{|c|c|c|c|c|c|}
\hline 202 & $0.123 E+04$ & 204. & 800. & 0.200 & 0.190 \\
\hline 203 & $0.119 E+04$ & 264. & 900. & 0.200 & 0.190 \\
\hline 204 & $0.117 \mathrm{E}+04$ & 306. & 983. & 0.300 & 0.190 \\
\hline 205 & $0.120 \mathrm{E}+04$ & 363. & $0.100 \mathrm{E}+04$ & 0.362 & 0.190 \\
\hline 206 & $0.123 E+04$ & 415. & $0.100 \mathrm{E}+04$ & 0.362 & 0.190 \\
\hline 207 & $0.119 E+04$ & 483. & $0.100 \mathrm{E}+04$ & 0.350 & 0.190 \\
\hline 208 & $0.119 E+04$ & 534. & $0.100 \mathrm{E}+04$ & 0.337 & 0.190 \\
\hline 209 & $0.119 E+04$ & 573. & $0.100 \mathrm{E}+04$ & 0.312 & 0.190 \\
\hline 210 & $0.120 \mathrm{E}+04$ & 642. & $0.100 \mathrm{E}+04$ & 0.300 & 0.190 \\
\hline 211 & $0.121 E+04$ & 715. & $0.100 \mathrm{E}+04$ & 0.300 & 0.190 \\
\hline 212 & $0.130 \mathrm{E}+04$ & 182. & 750. & 0.200 & 0.190 \\
\hline 213 & $0.129 \mathrm{E}+04$ & 212. & 800. & 0.200 & 0.190 \\
\hline 214 & $0.126 \mathrm{E}+04$ & 256. & 900. & 0.200 & 0.190 \\
\hline 215 & $0.126 \mathrm{E}+04$ & 290. & 975. & 0.225 & 0.190 \\
\hline 216 & $0.126 \mathrm{E}+04$ & 342. & $0.100 \mathrm{E}+04$ & 0.300 & 0.190 \\
\hline 217 & $0.128 \mathrm{E}+04$ & 398. & $0.100 \mathrm{E}+04$ & 0.350 & 0.190 \\
\hline 218 & $0.127 \mathrm{E}+04$ & 470. & $0.100 E+04$ & 0.350 & 0.190 \\
\hline 219 & $0.126 \mathrm{E}+04$ & 522. & $0.100 \mathrm{E}+04$ & 0.337 & 0.190 \\
\hline 220 & $0.125 E+04$ & 567. & $0.100 \mathrm{E}+04$ & 0.312 & 0.190 \\
\hline 221 & $0.126 \mathrm{E}+04$ & 634. & $0.100 \mathrm{E}+04$ & 0.300 & 0.190 \\
\hline 222 & $0.127 \mathrm{E}+04$ & 707. & $0.100 \mathrm{E}+04$ & 0.300 & 0.190 \\
\hline 223 & $0.136 \mathrm{E}+04$ & 188. & 750 & 0.200 & 0.190 \\
\hline 224 & $0.132 \mathrm{E}+04$ & 216. & 800. & 0.200 & 0.190 \\
\hline 225 & $0.133 E+04$ & 265. & 900. & 0.200 & 0.190 \\
\hline 226 & $0.136 \mathrm{E}+04$ & 302. & 900. & 0.233 & 0.177 \\
\hline 227 & $0.135 \mathrm{E}+04$ & 337. & 937. & 0.300 & 0.170 \\
\hline 228 & $0.134 \mathrm{E}+04$ & 397. & $0.100 \mathrm{E}+04$ & 0.350 & 0.170 \\
\hline 229 & $0.132 E+04$ & 459. & $0.100 \mathrm{E}+04$ & 0.350 & 0.170 \\
\hline 230 & $0.133 E+04$ & 514. & $0.100 \mathrm{E}+04$ & 0.325 & 0.170 \\
\hline 231 & $0.133 E+04$ & 557. & $0.100 \mathrm{E}+04$ & 0.300 & 0.170 \\
\hline 232 & $0.135 E+04$ & 603. & $0.100 \mathrm{E}+04$ & 0.300 & 0.170 \\
\hline 233 & $0.136 \mathrm{E}+04$ & 698. & $0.100 \mathrm{E}+04$ & 0.300 & 0.170 \\
\hline 234 & $0.140 \mathrm{E}+04$ & 307. & 750. & 0.300 & 0.150 \\
\hline 235 & $0.140 \mathrm{E}+04$ & 343. & 917. & 0.333 & 0.150 \\
\hline 236 & $0.141 E+04$ & 396. & $0.100 \mathrm{E}+04$ & 0.350 & 0.150 \\
\hline 237 & $0.139 \mathrm{E}+04$ & 447. & $0.100 \mathrm{E}+04$ & 0.362 & 0.150 \\
\hline 238 & $0.138 \mathrm{E}+04$ & 510. & $0.100 \mathrm{E}+04$ & 0.362 & 0.150 \\
\hline 239 & $0.137 \mathrm{E}+04$ & 553. & $0.100 \mathrm{E}+04$ & 0.350 & 0.150 \\
\hline 240 & $0.141 \mathrm{E}+04$ & 622. & $0.100 \mathrm{E}+04$ & 0.350 & 0.150 \\
\hline 241 & $0.141 E+04$ & 690. & $0.100 \mathrm{E}+04$ & 0.350 & 0.150 \\
\hline 242 & $0.146 \mathrm{E}+04$ & 380. & $0.100 \mathrm{E}+04$ & 0.300 & 0.150 \\
\hline 243 & $0.146 \mathrm{E}+04$ & 396. & $0.100 \mathrm{E}+04$ & 0.312 & 0.150 \\
\hline 244 & $0.147 \mathrm{E}+04$ & 433. & $0.100 \mathrm{E}+04$ & 0.362 & 0.150 \\
\hline 245 & $0.146 \mathrm{E}+04$ & 493. & $0.100 \mathrm{E}+04$ & 0.400 & 0.150 \\
\hline 246 & $0.145 E+04$ & 540. & $0.100 \mathrm{E}+04$ & 0.387 & 0.150 \\
\hline 247 & $0.149 E+04$ & 616. & $0.100 \mathrm{E}+04$ & 0.375 & 0.160 \\
\hline 248 & $0.153 \mathrm{E}+04$ & 678. & $0.100 \mathrm{E}+04$ & 0.375 & 0.170 \\
\hline 249 & $0.148 \mathrm{E}+04$ & 367. & $0.100 \mathrm{E}+04$ & 0.250 & 0.150 \\
\hline 250 & $0.149 E+04$ & 395. & $0.100 \mathrm{E}+04$ & 0.275 & 0.150 \\
\hline 251 & $0.150 \mathrm{E}+04$ & 427. & $0.100 \mathrm{E}+04$ & 0.350 & 0.150 \\
\hline 252 & $0.154 \mathrm{E}+04$ & 500. & $0.100 \mathrm{E}+04$ & 0.400 & 0.150 \\
\hline 253 & $0.156 \mathrm{E}+04$ & 539. & $0.100 \mathrm{E}+04$ & 0.375 & 0.150 \\
\hline
\end{tabular}




\begin{tabular}{|c|c|c|c|c|c|c|}
\hline 254 & $0.159 \mathrm{E}+04$ & 623. & $0.100 \mathrm{E}+04$ & 0.350 & 0.170 & \\
\hline 255 & $0.165 E+04$ & 648. & $0.100 \mathrm{E}+04$ & 0.350 & 0.190 & \\
\hline ELEMENT & $\Gamma \quad 1.0$ & 1.0 & 1. & 100 & 100 & 30. \\
\hline 1 & $0.51 E-04$ & $0.51 E-04$ & 1.0 & 1.0 & 1.0 & 1.0 \\
\hline 2 & $0.48 E-04$ & $0.48 E-04$ & 1.0 & 1.0 & 1.0 & 1.0 \\
\hline 3 & 0.81E-04 & 0.81E-04 & 1.0 & 1.0 & 1.0 & 1.0 \\
\hline 4 & $0.25 \mathrm{E}-04$ & $0.25 \mathrm{E}-04$ & 1.0 & 1.0 & 1.0 & 1.0 \\
\hline 5 & $0.73 E-04$ & 0.73E-04 & 1.0 & 1.0 & 1.0 & 1.0 \\
\hline 6 & $0.16 \mathrm{E}-03$ & $0.16 \mathrm{E}-03$ & 1.0 & 1.0 & 1.0 & 1.0 \\
\hline 7 & $0.50 \mathrm{E}-06$ & $0.50 \mathrm{E}-06$ & 1.0 & 1.0 & 1.0 & 1.0 \\
\hline 8 & $0.46 \mathrm{E}-04$ & $0.46 \mathrm{E}-04$ & 1.0 & 1.0 & 1.0 & 1.0 \\
\hline 9 & $0.46 \mathrm{E}-04$ & $0.46 \mathrm{E}-04$ & 1.0 & 1.0 & 1.0 & 1.0 \\
\hline 10 & $0.47 \mathrm{E}-04$ & $0.47 \mathrm{E}-04$ & 1.0 & 1.0 & 1.0 & 1.0 \\
\hline 11 & $0.54 \mathrm{E}-04$ & $0.54 \mathrm{E}-04$ & 1.0 & 1.0 & 1.0 & 1.0 \\
\hline 12 & $0.10 \mathrm{E}-03$ & $0.10 \mathrm{E}-03$ & 1.0 & 1.0 & 1.0 & 1.0 \\
\hline 13 & $0.13 E-03$ & $0.13 \mathrm{E}-03$ & 1.0 & 1.0 & 1.0 & 1.0 \\
\hline 14 & $0.11 E-03$ & $0.11 E-03$ & 1.0 & 1.0 & 1.0 & 1.0 \\
\hline 15 & $0.18 E-03$ & $0.18 \mathrm{E}-03$ & 1.0 & 1.0 & 1.0 & 1.0 \\
\hline 16 & $0.50 \mathrm{E}-06$ & $0.50 \mathrm{E}-06$ & 1.0 & 1.0 & 1.0 & 1.0 \\
\hline 17 & $0.46 \mathrm{E}-04$ & $0.46 \mathrm{E}-04$ & 1.0 & 1.0 & 1.0 & 1.0 \\
\hline 18 & $0.46 \mathrm{E}-04$ & $0.46 \mathrm{E}-04$ & 1.0 & 1.0 & 1.0 & 1.0 \\
\hline 19 & 0.89E-04 & 0.89E-04 & 1.0 & 1.0 & 1.0 & 1.0 \\
\hline 20 & $0.13 E-03$ & $0.13 E-03$ & 1.0 & 1.0 & 1.0 & 1.0 \\
\hline 21 & $0.30 \mathrm{E}-03$ & $0.30 \mathrm{E}-03$ & 1.0 & 1.0 & 1.0 & 1.0 \\
\hline 22 & $0.20 \mathrm{E}-03$ & $0.20 \mathrm{E}-03$ & 1.0 & 1.0 & 1.0 & 1.0 \\
\hline 23 & $0.15 E-03$ & $0.15 E-03$ & 1.0 & 1.0 & 1.0 & 1.0 \\
\hline 24 & $0.30 \mathrm{E}-06$ & $0.30 \mathrm{E}-06$ & 1.0 & 1.0 & 1.0 & 1.0 \\
\hline 25 & $0.18 \mathrm{E}-04$ & $0.18 \mathrm{E}-04$ & 1.0 & 1.0 & 1.0 & 1.0 \\
\hline 26 & $0.44 E-04$ & $0.44 \mathrm{E}-04$ & 1.0 & 1.0 & 1.0 & 1.0 \\
\hline 27 & $0.29 \mathrm{E}-03$ & $0.29 \mathrm{E}-03$ & 1.0 & 1.0 & 1.0 & 1.0 \\
\hline 28 & $0.42 \mathrm{E}-03$ & $0.42 \mathrm{E}-03$ & 1.0 & 1.0 & 1.0 & 1.0 \\
\hline 29 & $0.54 \mathrm{E}-03$ & $0.54 \mathrm{E}-03$ & 1.0 & 1.0 & 1.0 & 1.0 \\
\hline 30 & $0.78 \mathrm{E}-03$ & $0.78 \mathrm{E}-03$ & 1.0 & 1.0 & 1.0 & 1.0 \\
\hline 31 & $0.30 \mathrm{E}-06$ & $0.30 \mathrm{E}-06$ & 1.0 & 1.0 & 1.0 & 1.0 \\
\hline 32 & $0.58 \mathrm{E}-04$ & $0.58 \mathrm{E}-04$ & 1.0 & 1.0 & 1.0 & 1.0 \\
\hline 33 & $0.73 E-04$ & $0.73 E-04$ & 1.0 & 1.0 & 1.0 & 1.0 \\
\hline 34 & $0.15 E-03$ & $0.15 \mathrm{E}-03$ & 1.0 & 1.0 & 1.0 & 1.0 \\
\hline 35 & $0.57 \mathrm{E}-03$ & $0.57 \mathrm{E}-03$ & 1.0 & 1.0 & 1.0 & 1.0 \\
\hline 36 & $0.57 \mathrm{E}-03$ & $0.57 \mathrm{E}-03$ & 1.0 & 1.0 & 1.0 & 1.0 \\
\hline 37 & $0.57 \mathrm{E}-03$ & $0.57 E-03$ & 1.0 & 1.0 & 1.0 & 1.0 \\
\hline 38 & $0.40 \mathrm{E}-06$ & $0.40 \mathrm{E}-06$ & 1.0 & 1.0 & 1.0 & 1.0 \\
\hline 39 & 0.49E-04 & 0.49E-04 & 1.0 & 1.0 & 1.0 & 1.0 \\
\hline 40 & $0.42 \mathrm{E}-04$ & $0.42 \mathrm{E}-04$ & 1.0 & 1.0 & 1.0 & 1.0 \\
\hline 41 & $0.57 \mathrm{E}-03$ & $0.57 \mathrm{E}-03$ & 1.0 & 1.0 & 1.0 & 1.0 \\
\hline 42 & $0.51 \mathrm{E}-03$ & $0.51 E-03$ & 1.0 & 1.0 & 1.0 & 1.0 \\
\hline 43 & $0.56 \mathrm{E}-03$ & $0.56 \mathrm{E}-03$ & 1.0 & 1.0 & 1.0 & 1.0 \\
\hline 44 & $0.30 \mathrm{E}-06$ & $0.30 \mathrm{E}-06$ & 1.0 & 1.0 & 1.0 & 1.0 \\
\hline 45 & $0.13 \mathrm{E}-03$ & $0.13 E-03$ & 1.0 & 1.0 & 1.0 & 1.0 \\
\hline 46 & $0.58 \mathrm{E}-04$ & $0.58 \mathrm{E}-04$ & 1.0 & 1.0 & 1.0 & 1.0 \\
\hline 47 & $0.14 \mathrm{E}-03$ & $0.14 \mathrm{E}-03$ & 1.0 & 1.0 & 1.0 & 1.0 \\
\hline 48 & $0.24 \mathrm{E}-03$ & $0.24 \mathrm{E}-03$ & 1.0 & 1.0 & 1.0 & 1.0 \\
\hline 49 & $0.42 \mathrm{E}-03$ & $0.42 \mathrm{E}-03$ & 1.0 & 1.0 & 1.0 & 1.0 \\
\hline
\end{tabular}




\begin{tabular}{|c|c|c|c|c|c|c|}
\hline 50 & $0.40 \mathrm{E}-03$ & $0.40 \mathrm{E}-03$ & 1.0 & 1.0 & 1.0 & 1.0 \\
\hline 51 & $0.41 E-03$ & $0.41 E-03$ & 1.0 & 1.0 & 1.0 & 1.0 \\
\hline 52 & $0.30 \mathrm{E}-06$ & $0.30 \mathrm{E}-06$ & 1.0 & 1.0 & 1.0 & 1.0 \\
\hline 53 & $0.24 \mathrm{E}-03$ & $0.24 \mathrm{E}-03$ & 1.0 & 1.0 & 1.0 & 1.0 \\
\hline 54 & $0.79 \mathrm{E}-04$ & 0.79 E-04 & 1.0 & 1.0 & 1.0 & 1.0 \\
\hline 55 & $0.13 \mathrm{E}-03$ & $0.13 \mathrm{E}-03$ & 1.0 & 1.0 & 1.0 & 1.0 \\
\hline 56 & $0.20 \mathrm{E}-03$ & $0.20 \mathrm{E}-03$ & 1.0 & 1.0 & 1.0 & 1.0 \\
\hline 57 & $0.42 \mathrm{E}-03$ & $0.42 \mathrm{E}-03$ & 1.0 & 1.0 & 1.0 & 1.0 \\
\hline 58 & $0.47 \mathrm{E}-03$ & $0.47 \mathrm{E}-03$ & 1.0 & 1.0 & 1.0 & 1.0 \\
\hline 59 & $0.50 \mathrm{E}-03$ & $0.50 \mathrm{E}-03$ & 1.0 & 1.0 & 1.0 & 1.0 \\
\hline 60 & $0.40 \mathrm{E}-06$ & $0.40 \mathrm{E}-06$ & 1.0 & 1.0 & 1.0 & 1.0 \\
\hline 61 & $0.20 \mathrm{E}-03$ & $0.20 \mathrm{E}-03$ & 1.0 & 1.0 & 1.0 & 1.0 \\
\hline 62 & $0.81 E-05$ & $0.81 E-05$ & 1.0 & 1.0 & 1.0 & 1.0 \\
\hline 63 & $0.55 \mathrm{E}-03$ & $0.55 \mathrm{E}-03$ & 1.0 & 1.0 & 1.0 & 1.0 \\
\hline 64 & $0.59 E-03$ & $0.59 E-03$ & 1.0 & 1.0 & 1.0 & 1.0 \\
\hline 65 & $0.52 \mathrm{E}-03$ & $0.52 \mathrm{E}-03$ & 1.0 & 1.0 & 1.0 & 1.0 \\
\hline 66 & $0.57 \mathrm{E}-03$ & $0.57 \mathrm{E}-03$ & 1.0 & 1.0 & 1.0 & 1.0 \\
\hline 67 & $0.40 \mathrm{E}-06$ & $0.40 \mathrm{E}-06$ & 1.0 & 1.0 & 1.0 & 1.0 \\
\hline 68 & $0.56 \mathrm{E}-04$ & $0.56 \mathrm{E}-04$ & 1.0 & 1.0 & 1.0 & 1.0 \\
\hline 69 & $0.48 E-05$ & $0.48 E-05$ & 1.0 & 1.0 & 1.0 & 1.0 \\
\hline 70 & $0.51 \mathrm{E}-03$ & $0.51 \mathrm{E}-03$ & 1.0 & 1.0 & 1.0 & 1.0 \\
\hline 71 & $0.58 \mathrm{E}-03$ & $0.58 \mathrm{E}-03$ & 1.0 & 1.0 & 1.0 & 1.0 \\
\hline 72 & $0.63 \mathrm{E}-03$ & $0.63 \mathrm{E}-03$ & 1.0 & 1.0 & 1.0 & 1.0 \\
\hline 73 & $0.57 \mathrm{E}-03$ & $0.57 \mathrm{E}-03$ & 1.0 & 1.0 & 1.0 & 1.0 \\
\hline 74 & $0.40 \mathrm{E}-06$ & $0.40 \mathrm{E}-06$ & 1.0 & 1.0 & 1.0 & 1.0 \\
\hline 75 & $0.38 \mathrm{E}-04$ & $0.38 \mathrm{E}-04$ & 1.0 & 1.0 & 1.0 & 1.0 \\
\hline 76 & $0.68 \mathrm{E}-05$ & $0.68 \mathrm{E}-05$ & 1.0 & 1.0 & 1.0 & 1.0 \\
\hline 77 & $0.58 \mathrm{E}-03$ & $0.58 \mathrm{E}-03$ & 1.0 & 1.0 & 1.0 & 1.0 \\
\hline 78 & $0.53 \mathrm{E}-03$ & $0.53 \mathrm{E}-03$ & 1.0 & 1.0 & 1.0 & 1.0 \\
\hline 79 & $0.50 \mathrm{E}-03$ & $0.50 \mathrm{E}-03$ & 1.0 & 1.0 & 1.0 & 1.0 \\
\hline 80 & $0.54 \mathrm{E}-03$ & $0.54 \mathrm{E}-03$ & 1.0 & 1.0 & 1.0 & 1.0 \\
\hline 81 & $0.40 \mathrm{E}-06$ & $0.40 \mathrm{E}-06$ & 1.0 & 1.0 & 1.0 & 1.0 \\
\hline 82 & $0.12 \mathrm{E}-03$ & $0.12 E-03$ & 1.0 & 1.0 & 1.0 & 1.0 \\
\hline 83 & $0.53 \mathrm{E}-04$ & $0.53 E-04$ & 1.0 & 1.0 & 1.0 & 1.0 \\
\hline 84 & $0.56 \mathrm{E}-03$ & $0.56 \mathrm{E}-03$ & 1.0 & 1.0 & 1.0 & 1.0 \\
\hline 85 & $0.63 E-03$ & $0.63 \mathrm{E}-03$ & 1.0 & 1.0 & 1.0 & 1.0 \\
\hline 86 & $0.69 \mathrm{E}-03$ & $0.69 \mathrm{E}-03$ & 1.0 & 1.0 & 1.0 & 1.0 \\
\hline 87 & $0.53 \mathrm{E}-03$ & $0.53 \mathrm{E}-03$ & 1.0 & 1.0 & 1.0 & 1.0 \\
\hline 88 & $0.40 \mathrm{E}-06$ & $0.40 \mathrm{E}-06$ & 1.0 & 1.0 & 1.0 & 1.0 \\
\hline 89 & $0.38 \mathrm{E}-04$ & $0.38 \mathrm{E}-04$ & 1.0 & 1.0 & 1.0 & 1.0 \\
\hline 90 & $0.22 \mathrm{E}-04$ & $0.22 \mathrm{E}-04$ & 1.0 & 1.0 & 1.0 & 1.0 \\
\hline 91 & $0.32 \mathrm{E}-03$ & $0.32 \mathrm{E}-03$ & 1.0 & 1.0 & 1.0 & 1.0 \\
\hline 92 & $0.43 \mathrm{E}-03$ & $0.43 E-03$ & 1.0 & 1.0 & 1.0 & 1.0 \\
\hline 93 & $0.51 \mathrm{E}-03$ & $0.51 \mathrm{E}-03$ & 1.0 & 1.0 & 1.0 & 1.0 \\
\hline 94 & $0.52 \mathrm{E}-03$ & $0.52 \mathrm{E}-03$ & 1.0 & 1.0 & 1.0 & 1.0 \\
\hline 95 & $0.50 \mathrm{E}-03$ & $0.50 \mathrm{E}-03$ & 1.0 & 1.0 & 1.0 & 1.0 \\
\hline 96 & $0.40 \mathrm{E}-06$ & $0.40 \mathrm{E}-06$ & 1.0 & 1.0 & 1.0 & 1.0 \\
\hline 97 & $0.17 \mathrm{E}-04$ & $0.17 \mathrm{E}-04$ & 1.0 & 1.0 & 1.0 & 1.0 \\
\hline 98 & $0.34 \mathrm{E}-04$ & $0.34 \mathrm{E}-04$ & 1.0 & 1.0 & 1.0 & 1.0 \\
\hline 99 & $0.27 \mathrm{E}-03$ & $0.27 \mathrm{E}-03$ & 1.0 & 1.0 & 1.0 & 1.0 \\
\hline 100 & $0.40 \mathrm{E}-03$ & $0.40 \mathrm{E}-03$ & 1.0 & 1.0 & 1.0 & 1.0 \\
\hline 101 & $0.60 \mathrm{E}-03$ & $0.60 \mathrm{E}-03$ & 1.0 & 1.0 & 1.0 & 1.0 \\
\hline
\end{tabular}




\begin{tabular}{|c|c|c|c|c|c|c|}
\hline 102 & $0.74 E-03$ & $0.74 \mathrm{E}-03$ & 1.0 & 1.0 & 1.0 & 1.0 \\
\hline 103 & $0.74 \mathrm{E}-03$ & $0.74 \mathrm{E}-03$ & 1.0 & 1.0 & 1.0 & 1.0 \\
\hline 104 & $0.62 E-03$ & $0.62 \mathrm{E}-03$ & 1.0 & 1.0 & 1.0 & 1.0 \\
\hline 105 & $0.30 \mathrm{E}-06$ & $0.30 \mathrm{E}-06$ & 1.0 & 1.0 & 1.0 & 1.0 \\
\hline 106 & $0.15 E-04$ & $0.15 E-04$ & 1.0 & 1.0 & 1.0 & 1.0 \\
\hline 107 & $0.35 \mathrm{E}-04$ & $0.35 \mathrm{E}-04$ & 1.0 & 1.0 & 1.0 & 1.0 \\
\hline 108 & $0.32 \mathrm{E}-03$ & $0.32 \mathrm{E}-03$ & 1.0 & 1.0 & 1.0 & 1.0 \\
\hline 109 & $0.23 E-03$ & $0.23 \mathrm{E}-03$ & 1.0 & 1.0 & 1.0 & 1.0 \\
\hline 110 & $0.63 E-03$ & $0.63 \mathrm{E}-03$ & 1.0 & 1.0 & 1.0 & 1.0 \\
\hline 111 & $0.72 \mathrm{E}-03$ & $0.72 \mathrm{E}-03$ & 1.0 & 1.0 & 1.0 & 1.0 \\
\hline 112 & $0.81 E-03$ & $0.81 E-03$ & 1.0 & 1.0 & 1.0 & 1.0 \\
\hline 113 & $0.77 \mathrm{E}-03$ & $0.77 \mathrm{E}-03$ & 1.0 & 1.0 & 1.0 & 1.0 \\
\hline 114 & $0.64 \mathrm{E}-03$ & $0.64 \mathrm{E}-03$ & 1.0 & 1.0 & 1.0 & 1.0 \\
\hline 115 & $0.30 \mathrm{E}-06$ & $0.30 \mathrm{E}-06$ & 1.0 & 1.0 & 1.0 & 1.0 \\
\hline 116 & $0.18 \mathrm{E}-04$ & $0.18 \mathrm{E}-04$ & 1.0 & 1.0 & 1.0 & 1.0 \\
\hline 117 & $0.35 \mathrm{E}-04$ & $0.35 \mathrm{E}-04$ & 1.0 & 1.0 & 1.0 & 1.0 \\
\hline 118 & $0.40 \mathrm{E}-04$ & $0.40 \mathrm{E}-04$ & 1.0 & 1.0 & 1.0 & 1.0 \\
\hline 119 & $0.18 \mathrm{E}-03$ & $0.18 \mathrm{E}-03$ & 1.0 & 1.0 & 1.0 & 1.0 \\
\hline 120 & $0.27 \mathrm{E}-03$ & $0.27 \mathrm{E}-03$ & 1.0 & 1.0 & 1.0 & 1.0 \\
\hline 121 & $0.35 E-03$ & $0.35 E-03$ & 1.0 & 1.0 & 1.0 & 1.0 \\
\hline 122 & $0.52 \mathrm{E}-03$ & $0.52 \mathrm{E}-03$ & 1.0 & 1.0 & 1.0 & 1.0 \\
\hline 123 & $0.65 \mathrm{E}-03$ & $0.65 \mathrm{E}-03$ & 1.0 & 1.0 & 1.0 & 1.0 \\
\hline 124 & $0.77 E-03$ & $0.77 E-03$ & 1.0 & 1.0 & 1.0 & 1.0 \\
\hline 125 & $0.79 E-03$ & $0.79 E-03$ & 1.0 & 1.0 & 1.0 & 1.0 \\
\hline 126 & $0.76 \mathrm{E}-03$ & $0.76 \mathrm{E}-03$ & 1.0 & 1.0 & 1.0 & 1.0 \\
\hline 127 & $0.78 E-03$ & $0.78 E-03$ & 1.0 & 1.0 & 1.0 & 1.0 \\
\hline 128 & $0.22 E-05$ & $0.22 \mathrm{E}-05$ & 1.0 & 1.0 & 1.0 & 1.0 \\
\hline 129 & $0.80 \mathrm{E}-05$ & $0.80 \mathrm{E}-05$ & 1.0 & 1.0 & 1.0 & 1.0 \\
\hline 130 & $0.16 \mathrm{E}-04$ & $0.16 \mathrm{E}-04$ & 1.0 & 1.0 & 1.0 & 1.0 \\
\hline 131 & $0.75 E-03$ & $0.75 \mathrm{E}-03$ & 1.0 & 1.0 & 1.0 & 1.0 \\
\hline 132 & $0.79 \mathrm{E}-03$ & $0.79 \mathrm{E}-03$ & 1.0 & 1.0 & 1.0 & 1.0 \\
\hline 133 & $0.87 E-03$ & 0.87 E-03 & 1.0 & 1.0 & 1.0 & 1.0 \\
\hline 134 & $0.52 \mathrm{E}-03$ & $0.52 \mathrm{E}-03$ & 1.0 & 1.0 & 1.0 & 1.0 \\
\hline 135 & $0.46 \mathrm{E}-03$ & $0.46 \mathrm{E}-03$ & 1.0 & 1.0 & 1.0 & 1.0 \\
\hline 136 & $0.32 \mathrm{E}-03$ & $0.32 \mathrm{E}-03$ & 1.0 & 1.0 & 1.0 & 1.0 \\
\hline 137 & $0.63 E-05$ & $0.63 \mathrm{E}-05$ & 1.0 & 1.0 & 1.0 & 1.0 \\
\hline 138 & $0.98 E-05$ & $0.98 \mathrm{E}-05$ & 1.0 & 1.0 & 1.0 & 1.0 \\
\hline 139 & $0.98 \mathrm{E}-05$ & $0.98 \mathrm{E}-05$ & 1.0 & 1.0 & 1.0 & 1.0 \\
\hline 140 & $0.47 E-03$ & $0.47 \mathrm{E}-03$ & 1.0 & 1.0 & 1.0 & 1.0 \\
\hline 141 & $0.52 \mathrm{E}-03$ & $0.52 \mathrm{E}-03$ & 1.0 & 1.0 & 1.0 & 1.0 \\
\hline 142 & $0.62 \mathrm{E}-03$ & $0.62 \mathrm{E}-03$ & 1.0 & 1.0 & 1.0 & 1.0 \\
\hline 143 & $0.64 E-03$ & $0.64 \mathrm{E}-03$ & 1.0 & 1.0 & 1.0 & 1.0 \\
\hline 144 & $0.46 \mathrm{E}-03$ & $0.46 \mathrm{E}-03$ & 1.0 & 1.0 & 1.0 & 1.0 \\
\hline 145 & $0.11 E-03$ & $0.11 E-03$ & 1.0 & 1.0 & 1.0 & 1.0 \\
\hline 146 & $0.92 E-05$ & $0.92 E-05$ & 1.0 & 1.0 & 1.0 & 1.0 \\
\hline 147 & $0.16 \mathrm{E}-04$ & $0.16 \mathrm{E}-04$ & 1.0 & 1.0 & 1.0 & 1.0 \\
\hline 148 & $0.84 E-05$ & $0.84 \mathrm{E}-05$ & 1.0 & 1.0 & 1.0 & 1.0 \\
\hline 149 & $0.38 E-03$ & $0.38 \mathrm{E}-03$ & 1.0 & 1.0 & 1.0 & 1.0 \\
\hline 150 & $0.42 E-03$ & $0.42 E-03$ & 1.0 & 1.0 & 1.0 & 1.0 \\
\hline 151 & $0.56 \mathrm{E}-03$ & $0.56 \mathrm{E}-03$ & 1.0 & 1.0 & 1.0 & 1.0 \\
\hline 152 & $0.62 \mathrm{E}-03$ & $0.62 \mathrm{E}-03$ & 1.0 & 1.0 & 1.0 & 1.0 \\
\hline 153 & $0.44 E-03$ & $0.44 \mathrm{E}-03$ & 1.0 & 1.0 & 1.0 & 1.0 \\
\hline
\end{tabular}




\begin{tabular}{|c|c|c|c|c|c|c|}
\hline 154 & $0.16 \mathrm{E}-03$ & $0.16 \mathrm{E}-03$ & 1.0 & 1.0 & 1.0 & 1.0 \\
\hline 155 & $0.93 E-04$ & 0.93E-04 & 1.0 & 1.0 & 1.0 & 1.0 \\
\hline 156 & $0.31 E-04$ & $0.31 E-04$ & 1.0 & 1.0 & 1.0 & 1.0 \\
\hline 157 & $0.24 \mathrm{E}-04$ & $0.24 \mathrm{E}-04$ & 1.0 & 1.0 & 1.0 & 1.0 \\
\hline 158 & $0.45 \mathrm{E}-03$ & $0.45 \mathrm{E}-03$ & 1.0 & 1.0 & 1.0 & 1.0 \\
\hline 159 & $0.81 E-03$ & $0.81 \mathrm{E}-03$ & 1.0 & 1.0 & 1.0 & 1.0 \\
\hline 160 & 0.83E-03 & $0.83 E-03$ & 1.0 & 1.0 & 1.0 & 1.0 \\
\hline 161 & $0.58 \mathrm{E}-03$ & $0.58 \mathrm{E}-03$ & 1.0 & 1.0 & 1.0 & 1.0 \\
\hline 162 & $0.46 \mathrm{E}-03$ & $0.46 \mathrm{E}-03$ & 1.0 & 1.0 & 1.0 & 1.0 \\
\hline 163 & $0.32 \mathrm{E}-03$ & $0.32 \mathrm{E}-03$ & 1.0 & 1.0 & 1.0 & 1.0 \\
\hline 164 & 0.79E-03 & $0.79 E-03$ & 1.0 & 1.0 & 1.0 & 1.0 \\
\hline 165 & $0.77 \mathrm{E}-03$ & $0.77 \mathrm{E}-03$ & 1.0 & 1.0 & 1.0 & 1.0 \\
\hline 166 & $0.67 \mathrm{E}-03$ & $0.67 \mathrm{E}-03$ & 1.0 & 1.0 & 1.0 & 1.0 \\
\hline 167 & $0.68 \mathrm{E}-03$ & $0.68 \mathrm{E}-03$ & 1.0 & 1.0 & 1.0 & 1.0 \\
\hline 168 & $0.48 \mathrm{E}-03$ & $0.48 \mathrm{E}-03$ & 1.0 & 1.0 & 1.0 & 1.0 \\
\hline 169 & $0.31 \mathrm{E}-03$ & $0.31 \mathrm{E}-03$ & 1.0 & 1.0 & 1.0 & 1.0 \\
\hline 170 & $0.37 \mathrm{E}-03$ & $0.37 \mathrm{E}-03$ & 1.0 & 1.0 & 1.0 & 1.0 \\
\hline 171 & $0.20 \mathrm{E}-03$ & $0.20 \mathrm{E}-03$ & 1.0 & 1.0 & 1.0 & 1.0 \\
\hline 172 & $0.27 \mathrm{E}-03$ & $0.27 \mathrm{E}-03$ & 1.0 & 1.0 & 1.0 & 1.0 \\
\hline 173 & $0.22 \mathrm{E}-03$ & $0.22 \mathrm{E}-03$ & 1.0 & 1.0 & 1.0 & 1.0 \\
\hline 174 & $0.61 E-03$ & $0.61 \mathrm{E}-03$ & 1.0 & 1.0 & 1.0 & 1.0 \\
\hline 175 & $0.59 \mathrm{E}-03$ & $0.59 \mathrm{E}-03$ & 1.0 & 1.0 & 1.0 & 1.0 \\
\hline 176 & $0.62 E-03$ & $0.62 \mathrm{E}-03$ & 1.0 & 1.0 & 1.0 & 1.0 \\
\hline 177 & $0.49 \mathrm{E}-03$ & $0.49 \mathrm{E}-03$ & 1.0 & 1.0 & 1.0 & 1.0 \\
\hline 178 & $0.46 \mathrm{E}-03$ & $0.46 \mathrm{E}-03$ & 1.0 & 1.0 & 1.0 & 1.0 \\
\hline 179 & $0.32 \mathrm{E}-03$ & $0.32 \mathrm{E}-03$ & 1.0 & 1.0 & 1.0 & 1.0 \\
\hline 180 & $0.21 E-03$ & $0.21 E-03$ & 1.0 & 1.0 & 1.0 & 1.0 \\
\hline 181 & $0.20 \mathrm{E}-03$ & $0.20 \mathrm{E}-03$ & 1.0 & 1.0 & 1.0 & 1.0 \\
\hline 182 & $0.27 \mathrm{E}-03$ & $0.27 \mathrm{E}-03$ & 1.0 & 1.0 & 1.0 & 1.0 \\
\hline 183 & $0.29 \mathrm{E}-03$ & $0.29 \mathrm{E}-03$ & 1.0 & 1.0 & 1.0 & 1.0 \\
\hline 184 & $0.72 E-03$ & $0.72 E-03$ & 1.0 & 1.0 & 1.0 & 1.0 \\
\hline 185 & $0.35 \mathrm{E}-03$ & $0.35 E-03$ & 1.0 & 1.0 & 1.0 & 1.0 \\
\hline 186 & $0.46 \mathrm{E}-03$ & $0.46 \mathrm{E}-03$ & 1.0 & 1.0 & 1.0 & 1.0 \\
\hline 187 & $0.61 E-03$ & $0.61 \mathrm{E}-03$ & 1.0 & 1.0 & 1.0 & 1.0 \\
\hline 188 & $0.73 E-03$ & $0.73 E-03$ & 1.0 & 1.0 & 1.0 & 1.0 \\
\hline 189 & $0.43 E-03$ & $0.43 E-03$ & 1.0 & 1.0 & 1.0 & 1.0 \\
\hline 190 & $0.20 \mathrm{E}-03$ & $0.20 \mathrm{E}-03$ & 1.0 & 1.0 & 1.0 & 1.0 \\
\hline 191 & $0.27 \mathrm{E}-03$ & $0.27 \mathrm{E}-03$ & 1.0 & 1.0 & 1.0 & 1.0 \\
\hline 192 & $0.22 \mathrm{E}-03$ & $0.22 \mathrm{E}-03$ & 1.0 & 1.0 & 1.0 & 1.0 \\
\hline 193 & $0.32 \mathrm{E}-03$ & $0.32 \mathrm{E}-03$ & 1.0 & 1.0 & 1.0 & 1.0 \\
\hline 194 & $0.46 \mathrm{E}-03$ & $0.46 \mathrm{E}-03$ & 1.0 & 1.0 & 1.0 & 1.0 \\
\hline 195 & $0.56 \mathrm{E}-03$ & $0.56 \mathrm{E}-03$ & 1.0 & 1.0 & 1.0 & 1.0 \\
\hline 196 & $0.44 E-03$ & $0.44 \mathrm{E}-03$ & 1.0 & 1.0 & 1.0 & 1.0 \\
\hline 197 & $0.21 E-03$ & $0.21 E-03$ & 1.0 & 1.0 & 1.0 & 1.0 \\
\hline 198 & $0.32 \mathrm{E}-03$ & $0.32 \mathrm{E}-03$ & 1.0 & 1.0 & 1.0 & 1.0 \\
\hline 199 & $0.37 \mathrm{E}-03$ & $0.37 \mathrm{E}-03$ & 1.0 & 1.0 & 1.0 & 1.0 \\
\hline 200 & $0.57 \mathrm{E}-03$ & $0.57 \mathrm{E}-03$ & 1.0 & 1.0 & 1.0 & 1.0 \\
\hline 201 & $0.48 \mathrm{E}-03$ & $0.48 \mathrm{E}-03$ & 1.0 & 1.0 & 1.0 & 1.0 \\
\hline 202 & $0.45 \mathrm{E}-03$ & $0.45 \mathrm{E}-03$ & 1.0 & 1.0 & 1.0 & 1.0 \\
\hline 203 & $0.22 \mathrm{E}-03$ & $0.22 \mathrm{E}-03$ & 1.0 & 1.0 & 1.0 & 1.0 \\
\hline 204 & $0.22 \mathrm{E}-03$ & $0.22 \mathrm{E}-03$ & 1.0 & 1.0 & 1.0 & 1.0 \\
\hline 205 & $0.27 \mathrm{E}-03$ & $0.27 E-03$ & 1.0 & 1.0 & 1.0 & 1.0 \\
\hline
\end{tabular}


Appendix 9: SUTRA unit 5 input file for simulation shown in figures 18 and 19--Continued

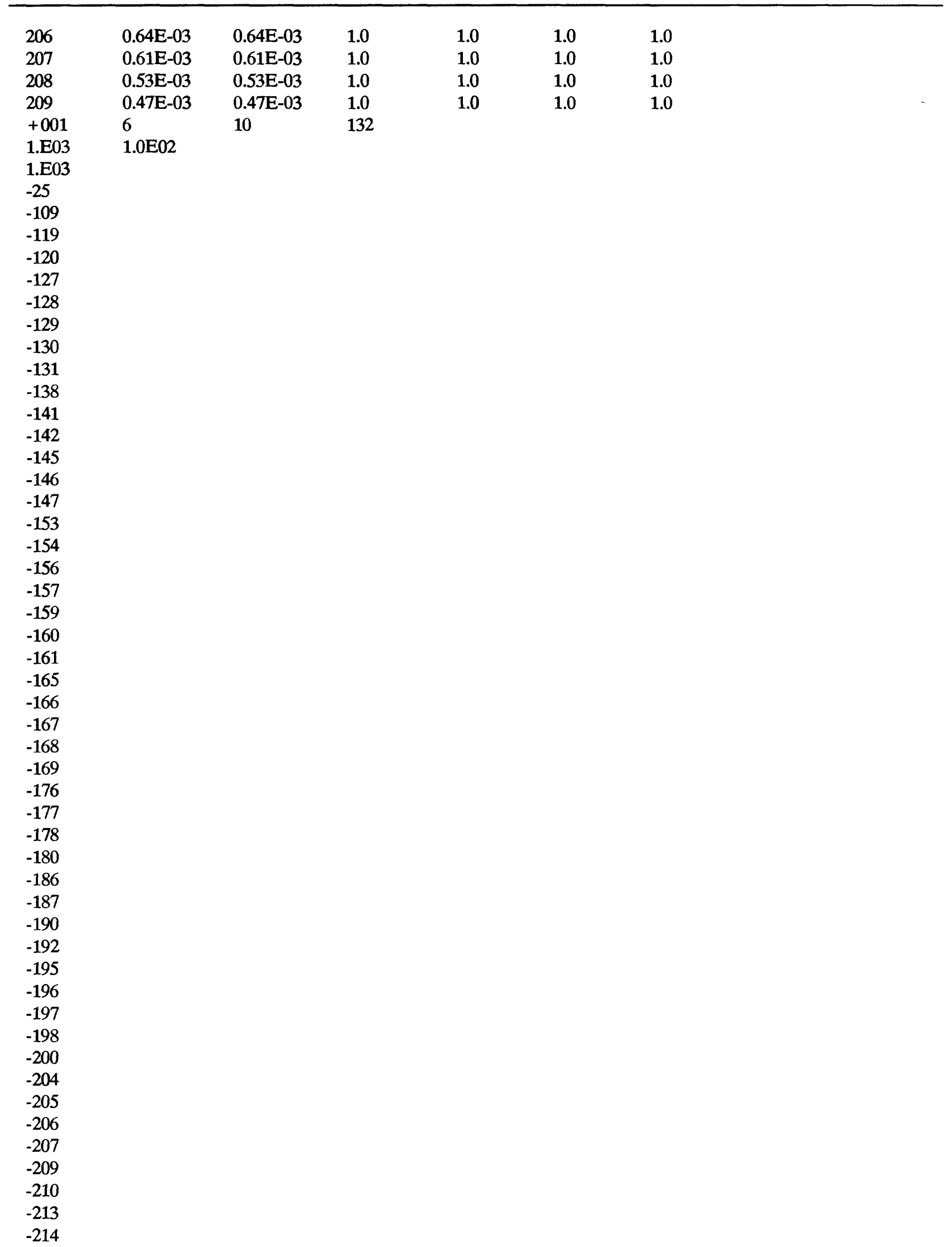




$\begin{array}{lll}5.52 & 200 . & \text { San Gorgonio } \\ 4.14 & 200 . & \text { San Gorgonio } \\ 2.76 & 200 . & \text { San Gorgonio } \\ 1.86 & 200 . & \text { Snow ck } \\ 1.86 & 200 . & \text { Snow ck } \\ 0.655 & 200 . & \text { Falls ck } \\ 0.655 & 200 . & \text { Falls ck } \\ 0.167 & 200 . & \text { Banning fault } \\ 0.167 & 200 . & \text { Banning fault }\end{array}$

AR + WW Rv. above bridge $A R+W W$ riv below bridge Banning fault

$\mathrm{AR}+\mathrm{WW}$ riv below bridge

Banning fault

$\mathrm{AR}+\mathrm{WW}$ riv below bridge

Banning fault

$\mathrm{AR}+\mathrm{WW}$ riv below bridge

Banning fault

$A R+W W$ river

Banning fault

AR

AR

AR

Banning fault

AR

AR

AR

AR

AR 


\begin{tabular}{|c|c|c|c|}
\hline-94 & & & AR \\
\hline 90 & $0.414 \mathrm{E}-01$ & 200. & Banning fault \\
\hline 98 & $0.290 \mathrm{E}-01$ & 200. & Banning fault \\
\hline 106 & 0.138 & 200. & Banning fault \\
\hline 115 & $0.842 \mathrm{E}-01$ & 200. & Banning fault \\
\hline 107 & 1.38 & 200. & Chino \\
\hline 117 & 1.38 & 200. & Chino \\
\hline 125 & $0.483 E-01$ & 200. & Banning fault \\
\hline 126 & 3.17 & 200. & Tahquitz \\
\hline 136 & $0.483 E-01$ & 200. & Banning fault \\
\hline 139 & 1.38 & 200. & Andreas \\
\hline 150 & 0.103 & 200. & Banning fault \\
\hline 137 & 1.03 & 200. & Palm Canyon \\
\hline 151 & 1.03 & 200. & Palm Canyon \\
\hline 152 & 0.35 & 200. & Palm Canyon \\
\hline 164 & 0.241 & 200. & Banning fault \\
\hline 174 & 0.152 & 200. & Banning fault \\
\hline 184 & 0.124 & 200. & Banning fault \\
\hline 211 & 0.166 & 200. & Banning fault \\
\hline 201 & 0.46 & 200. & Deep ck \\
\hline 202 & 0.46 & 200. & Deep ck \\
\hline 212 & 0.46 & 200. & Deep ck \\
\hline 222 & 0.193 & 200. & Banning fault \\
\hline 233 & 0.117 & 200. & Banning fault \\
\hline 248 & 0.166 & 200. & Banning fault \\
\hline
\end{tabular}

$-249$

\begin{tabular}{|c|c|c|c|c|c|c|c|}
\hline & 10 & & & & & & \\
\hline 24 & 25 & 26 & 27 & 129 & 130 & 131 & 132 \\
\hline 1 & 1 & 11 & 12 & 2 & & & \\
\hline 2 & 2 & 12 & 13 & 3 & & & \\
\hline 3 & 3 & 13 & 14 & 4 & & & \\
\hline 4 & 4 & 14 & 15 & 5 & & & \\
\hline 5 & 5 & 15 & 16 & 6 & & & \\
\hline 6 & 6 & 16 & 17 & 7 & & & \\
\hline 7 & 7 & 17 & 18 & 8 & & & \\
\hline 8 & 8 & 18 & 19 & 9 & & & \\
\hline 9 & 9 & 19 & 20 & 10 & & & \\
\hline 10 & 11 & 21 & 22 & 12 & & & \\
\hline 11 & 12 & 22 & 23 & 13 & & & \\
\hline 12 & 13 & 23 & 24 & 14 & & & \\
\hline 13 & 14 & 24 & 25 & 15 & & & \\
\hline 14 & 15 & 25 & 26 & 16 & & & \\
\hline 15 & 16 & 26 & 27 & 17 & & & \\
\hline 16 & 17 & 27 & 28 & 18 & & & \\
\hline 17 & 18 & 28 & 29 & 19 & & & \\
\hline 18 & 19 & 29 & 30 & 20 & & & \\
\hline 19 & 22 & 31 & 32 & 23 & & & \\
\hline
\end{tabular}


Appendix 9: SUTRA unit 5 input file for simulation shown in figures 18 and 19--Continued

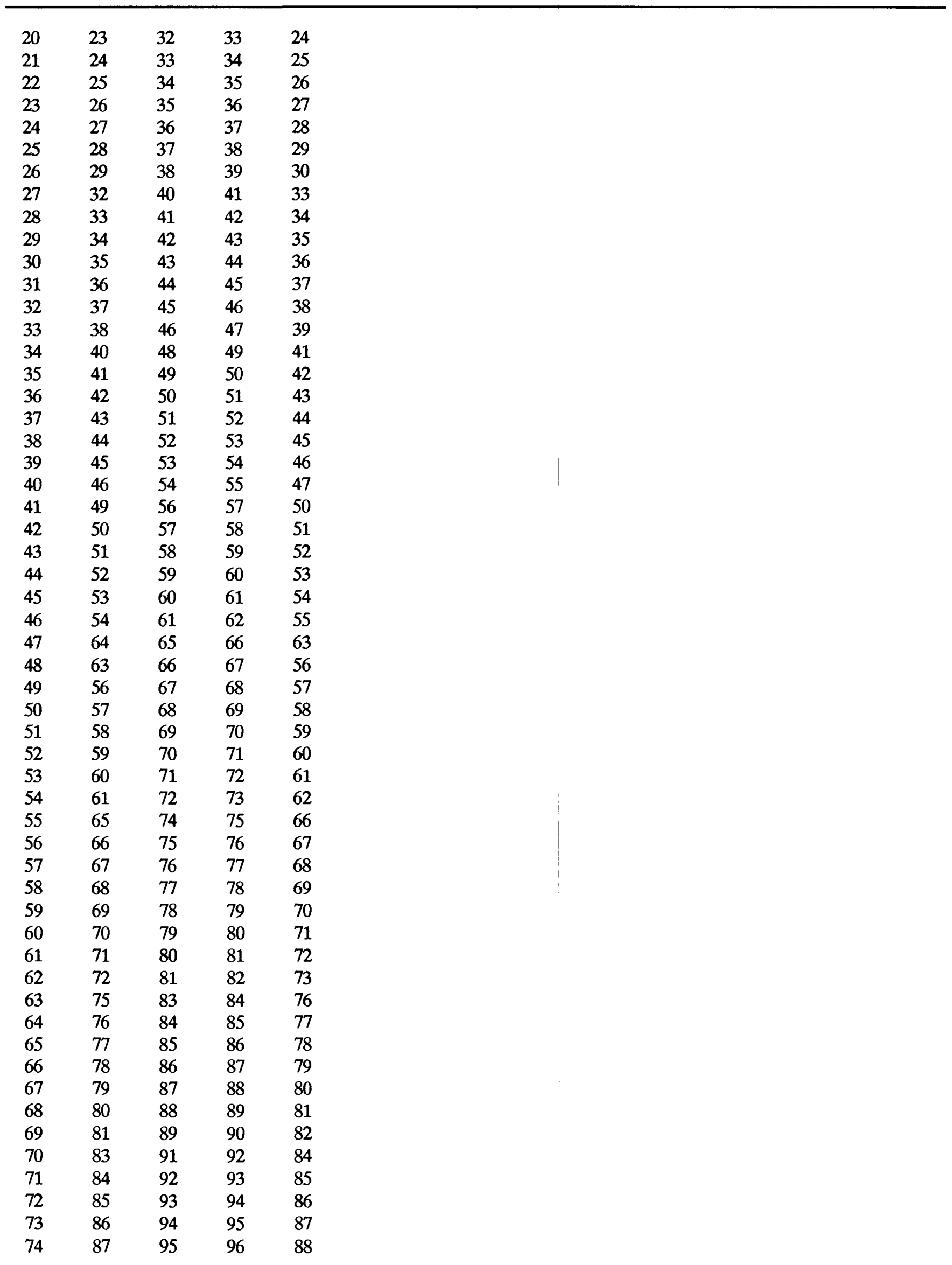

98 Evaluation of a Ground-Water Flow and Transport Model in the Upper Coachella Valley, California 


\begin{tabular}{rrrrr}
\hline 75 & 88 & 96 & 97 & 89 \\
76 & 89 & 97 & 98 & 90 \\
77 & 91 & 99 & 00 & 92 \\
78 & 92 & 100 & 101 & 93 \\
79 & 93 & 101 & 102 & 94 \\
80 & 94 & 102 & 103 & 95 \\
81 & 95 & 103 & 104 & 96 \\
82 & 96 & 104 & 105 & 97 \\
83 & 97 & 105 & 106 & 98 \\
84 & 99 & 108 & 109 & 100 \\
85 & 100 & 109 & 110 & 101 \\
86 & 101 & 110 & 111 & 102 \\
87 & 102 & 111 & 112 & 103 \\
88 & 103 & 112 & 113 & 104 \\
89 & 104 & 113 & 114 & 105 \\
90 & 105 & 114 & 115 & 106 \\
91 & 107 & 117 & 118 & 108 \\
92 & 108 & 118 & 119 & 109 \\
93 & 109 & 119 & 120 & 110 \\
94 & 110 & 120 & 121 & 111 \\
95 & 111 & 121 & 122 & 112 \\
96 & 112 & 122 & 123 & 113 \\
97 & 113 & 123 & 124 & 114 \\
98 & 114 & 124 & 125 & 115 \\
99 & 116 & 127 & 128 & 117 \\
100 & 117 & 128 & 129 & 118 \\
101 & 118 & 129 & 130 & 119 \\
102 & 119 & 130 & 131 & 120 \\
103 & 120 & 131 & 132 & 121 \\
104 & 121 & 132 & 133 & 122 \\
105 & 122 & 133 & 134 & 123 \\
106 & 123 & 134 & 135 & 124 \\
107 & 124 & 135 & 136 & 125 \\
108 & 126 & 140 & 141 & 127 \\
109 & 127 & 141 & 142 & 128 \\
110 & 128 & 142 & 143 & 129 \\
111 & 129 & 143 & 144 & 130 \\
112 & 130 & 144 & 145 & 131 \\
113 & 131 & 145 & 146 & 132 \\
114 & 132 & 146 & 147 & 133 \\
115 & 133 & 147 & 148 & 134 \\
116 & 134 & 148 & 149 & 135 \\
117 & 135 & 149 & 150 & 136 \\
118 & 137 & 151 & 152 & 138 \\
119 & 138 & 152 & 153 & 139 \\
120 & 139 & 153 & 154 & 140 \\
121 & 140 & 154 & 155 & 141 \\
122 & 141 & 155 & 156 & 142 \\
123 & 142 & 156 & 157 & 143 \\
124 & 143 & 157 & 158 & 144 \\
125 & 144 & 158 & 159 & 145 \\
126 & 145 & 159 & 160 & 146 \\
127 & 146 & 160 & 161 & 147 \\
128 & 147 & 161 & 162 & 148 \\
129 & 148 & 162 & 163 & 149
\end{tabular}




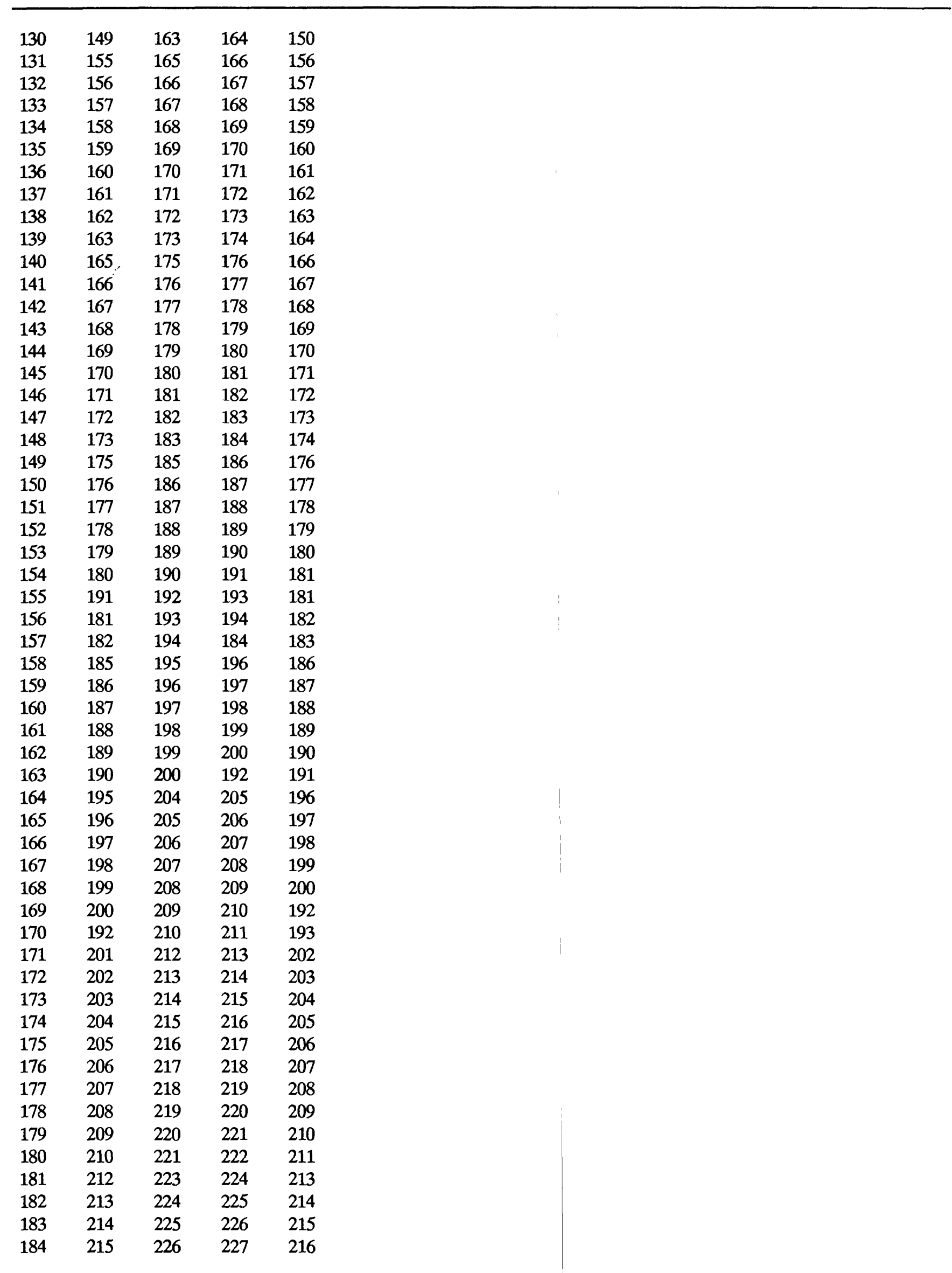


Appendix 9: SUTRA unit 5 input file for simulation shown in figures 18 and 19--Continued

\begin{tabular}{lllll}
\hline 185 & 216 & 227 & 228 & 217 \\
186 & 217 & 228 & 229 & 218 \\
187 & 218 & 229 & 230 & 219 \\
188 & 219 & 230 & 231 & 220 \\
189 & 220 & 231 & 232 & 221 \\
190 & 221 & 232 & 233 & 222 \\
191 & 226 & 234 & 235 & 227 \\
192 & 227 & 235 & 236 & 228 \\
193 & 228 & 236 & 237 & 229 \\
194 & 229 & 237 & 238 & 230 \\
195 & 230 & 238 & 239 & 231 \\
196 & 231 & 239 & 240 & 232 \\
197 & 232 & 240 & 241 & 233 \\
198 & 235 & 242 & 243 & 236 \\
199 & 236 & 243 & 244 & 237 \\
200 & 237 & 244 & 245 & 238 \\
201 & 238 & 245 & 246 & 239 \\
202 & 239 & 246 & 247 & 240 \\
203 & 240 & 247 & 248 & 241 \\
204 & 242 & 249 & 250 & 243 \\
205 & 243 & 250 & 251 & 244 \\
206 & 244 & 251 & 252 & 245 \\
207 & 245 & 252 & 253 & 246 \\
208 & 246 & 253 & 254 & 247 \\
209 & 247 & 254 & 255 & 248 \\
& & & & \\
\hline
\end{tabular}

